\title{
Nucleo-cytoskeletal interactions in the mechanical functioning of the cell
}

Citation for published version (APA):

Houben, F. (2011). Nucleo-cytoskeletal interactions in the mechanical functioning of the cell. [Doctoral Thesis, Maastricht University]. Maastricht University. https://doi.org/10.26481/dis.20111220fh

Document status and date:

Published: 01/01/2011

DOI:

10.26481/dis.20111220fh

Document Version:

Publisher's PDF, also known as Version of record

\section{Please check the document version of this publication:}

- A submitted manuscript is the version of the article upon submission and before peer-review. There can be important differences between the submitted version and the official published version of record.

People interested in the research are advised to contact the author for the final version of the publication, or visit the DOI to the publisher's website.

- The final author version and the galley proof are versions of the publication after peer review.

- The final published version features the final layout of the paper including the volume, issue and page numbers.

Link to publication

\footnotetext{
General rights rights.

- You may freely distribute the URL identifying the publication in the public portal. please follow below link for the End User Agreement:

www.umlib.nl/taverne-license

Take down policy

If you believe that this document breaches copyright please contact us at:

repository@maastrichtuniversity.nl

providing details and we will investigate your claim.
}

Copyright and moral rights for the publications made accessible in the public portal are retained by the authors and/or other copyright owners and it is a condition of accessing publications that users recognise and abide by the legal requirements associated with these

- Users may download and print one copy of any publication from the public portal for the purpose of private study or research.

- You may not further distribute the material or use it for any profit-making activity or commercial gain

If the publication is distributed under the terms of Article $25 \mathrm{fa}$ of the Dutch Copyright Act, indicated by the "Taverne" license above, 


\section{Nucleo-cytoskeletal interactions in the mechanical functioning of the cell}

Frederik Houben 
Nucleo-cytoskeletal interactions in the mechanical functioning of the cell Frederik Houben

Cover design by Cathy Willem

Printed by Parys Printing

(C) Frederik Houben, Maastricht 2011 


\section{Nucleo-cytoskeletal interactions in the mechanical functioning of the cell}

\section{Proefschrift}

Ter verkrijging van de graad van doctor aan de Universiteit Maastricht, op gezag van de Rector Magnificus, Prof. mr. G.P.M.F. Mols

volgens het besluit van het college van Decanen, in het openbaar te verdedigen op dinsdag 20 december 2011 om 10.00 uur

door

\section{Frederik Houben}

Geboren te Herk-de-Stad op 22 oktober 1979 


\section{PROMOTORES}

Prof. Dr. Frans Ramaekers

Prof. Dr. Luc Snoeckx (TU/e - UM)

\section{COPROMOTOR}

Dr. Jos Broers

\section{BEOORDELINGSCOMMISSIE}

Prof. Dr. E. Mariman, voorzitter

Dr. W.M. Blankesteijn

Prof. Dr. J.P.M. Geraedts

Prof. Dr. C.J. Hutchison (Durham, UK)

Dr. A. Sonnenberg (NKI, Amsterdam)

The study described in this thesis was performed within the School for Cardiovascular Diseases (CARIM) and supported by a grant of the Transnational University of Limburg 


\section{Contents}

General Introduction

Chapter 1: Role of nuclear lamina-cytoskeleton interactions in the maintenance of cellular strength.

Biochimica Biophysica Acta - Molecular Cell Research 2007; 1773; 675-686

Chapter 2: Disturbed nuclear orientation and cellular migration in A-type lamin deficient cells.

Biochimica Biophysica Acta - Molecular Cell Research 2009; 1793; 312-324

Chapter 3: Increased plasticity of the nuclear envelope and hypermobility of telomeres due to the loss of A-type lamins.

Biochimica Biophysica Acta - General Subjects 2010; 1800; 448-458

Chapter 4: Repetitive disruptions of the nuclear envelope invoke temporary loss of cellular compartmentalization in laminopathies.

Human Molecular Genetics 2011; 20; 4175-4186

Chapter 5: Cytoplasmic localization of PML nuclear bodies in laminopathies.

In preparation

Summary and General Discussion

Samenvatting

Curriculum Vitae

List of Publications

Dankwoord 


\section{General Introduction}


The Encarta World English Dictionary defines the nucleus as: the central body, usually spherical, within a eukaryotic cell, which is a membraneencased mass of protoplasm containing the chromosomes and other genetic information necessary to control cell growth and reproduction [1].

This definition exemplifies the hitherto generally accepted view of the nucleus as an oval-shaped, standalone container for the genetic material, which forms the base for all life. The interaction of the nucleus with its surroundings, via the nuclear membrane appears to be of minor interest. In this general view, the nuclear membrane is only a thin fleece, with one main function, separating the DNA from the rest of the cell.

When older study books discuss the function of the nuclear membrane more in detail, the nuclear lamina is often mentioned. It is described as important for maintaining the nuclear shape [2]. But the nucleus still remains characterized as a cell organelle, which floats almost freely inside the nucleus. The nucleus and the cytoplasm are seen as two compartments, which influence each other only through signalling proteins.

However, the last couple of years, it has become clear that the nucleus does not only interact with the rest of the cell through signalling pathways, but that it is also physically connected to the different elements of the cytoskeleton. One of the functions of these physical connections is withstanding external mechanical forces which are exerted onto the cells [3]. In turn, cells are not independent entities; they are part of tissues, organs, and whole organisms. Movement of any part of an organism will lead to mechanical stress, exerted onto the cells, and thus, the nucleus. To protect the genetic material from the external mechanical forces, it is essential that it is shielded from these forces. The nuclear envelope plays a major role in this protection mechanism. It consists of the nuclear membrane, the nuclear lamina and associated proteins (NETs).

The nuclear lamina can be found just below the nuclear membrane, it is a meshwork of lamin filaments. This structure provides structural support and gives stiffness to the membrane. This prevents the nucleus from collapsing during compression, as its gives resistance to the external mechanical stress $[36,37,50]$. Lamins are type $\mathrm{V}$ intermediate filament. They can be subdivided into 2 main types, the A-type lamins (lamins A, A $\Delta 10, \mathrm{C}$ and $\mathrm{C} 2$ ) and the B-type lamins (lamins B1, B2 and B3). While B-type lamins are expressed in the majority of cells, the expression of A-type lamins is linked to the differentiation process. B-type lamins form the fundamental component of the nuclear lamina (and are thus essential for cell survival) while A-type 
lamins are needed for other cellular processes like gene regulation [4]. Lamin proteins need extensive processing after translation before they can be incorporated into the lamina, including farnesylation, and two cleavage steps in the case of lamin A. Processing of lamin A is essential for its correct incorporation into the nuclear lamina (for a review see [5]). Cells containing unprocessed lamins show severe functional defects, resulting in diseases such as progeria (see below).

Several nuclear transmembrane proteins (nuclear envelope transmembrane proteins: NET) are associated with lamins, At the inside of the nuclear membrane, lamins have an important anchorage function for other NETs [6]. A number of these proteins (SUN, Nesprin) form complexes throughout the nuclear membrane and link at the cytoplasmic side to the different elements of the cytoskeleton: nesprin- 1 and 2 bind to actin [7,8], nesprin-3 binds to intermediate filaments (with plectin as the linker protein) [9] and nesprin-4 links to tubulin (with kinesin-1 as the linker protein) [10]. This is called the LInker of Nucleoskeleton and Cytoskeleton complex (LINC) [11]. Through this LINC-complex, the nuclear lamina and the cellular cytoskeleton form a mechanical scaffold, which protects the cell from mechanical stress. The nucleus lies encapsulated in this scaffold.

This structure combines internal compression (microtubules) and tension (actin filaments) forces and protects the cellular components (like the nucleus) during mechanical compression [3, 12-14]. All the elements of this mechanical network are essential, as they form one interacting mechanical entity, which cannot function if one of the elements fails. These findings shift the depiction of the nucleus from a free-floating bubble to an integrative cellular organelle with a certain stiffness, which is physically connected to the rest of the cell.

The function of lamins is not limited to the mechanical task. At the inside of the nuclear membrane, lamins have an important scaffold function, which extends from the lamina deep into the nucleolus. Several nuclear factors which affect gene regulation (e.g. SREBP1 [15, 16], pRB [17-20], ERK [21, 22] become, after nuclear import, tethered to lamins and in general become inactivated until released from the lamin scaffold. An overview of the different nuclear factors and pathways influenced by lamins is given by Andres et al. [23].

The critical role of the LINC complex, including lamins, both in gene regulation and in mechanical scaffolding, can be observed when this system does not function properly. When mutations occur in any of the LINC, NET 
or lamina genes, pathologies occur. These pathologies are collectively termed either nuclear envelopathies if mutations arise in INM or NPC proteins [2426], or laminopathies if mutations arise in lamins [27-31]), in the genes of some lamin-associated proteins or in the genes of prelamin processing enzymes (e.g. Zmpste24). Based on the affected tissues, we can divide them in several groups:

i. Striated muscle disorders: Emery-Dreifuss muscular dystrophy, dilated cardiomyopathy, limb-girdle muscular dystrophy, hearthand syndrome.

ii. Partial lipodystrophy syndromes: Dunnigan-type familial partial lipodystrophy, mandibuloacral dysplasia, Barraquer-Simons syndrome

iii. Peripheral neuropathies: Charcot-Marie Tooth disease

iv. Progeroid syndromes: Hutchison-Gilford progeria syndrome, atypical Werner syndrome, mandibuloacral dysplasia, restrictive dermopathy and other progeroid syndromes.

v. Other syndromes: leukodystrophy, Pelger-Huët anomaly, Greenberg skeletal dysplasia, osteopoikilosis, sclerosing bone dysplasia, Busche-Ollendorf syndrome, nonsporadic melorheostosis, cerebellar ataxia, DYT1 dystonia

An overview of the different laminopathies can be found in reviews by Worman et al $[32,33]$. A database of all registered LMNA mutations can be found at http://www.umd.be/LMNA/ .

Several hypotheses regarding the mechanisms that underlie the different pathologies are formulated.

- The gene expression hypothesis stating that mutations of lamin leads to altered gene expression, as lamin $\mathrm{A} / \mathrm{C}$ proteins can act as gene transcription modulators [34]. An overview of the different nuclear factors and pathways affected in laminopathies is given by Maraldi et al. [35].

- The structural hypothesis: this hypothesis states that lamins are an essential part of a structural cellular network that is responsible for the mechanical integrity of cells when subjected to mechanical stress [30, 36-39]. The effects can lead to a more rigid or a more flexible nuclear envelope:

- When the processing of lamins is incomplete, due to a mutation in the lamin $\mathrm{A} / \mathrm{C}$ gene or in the genes of prelamin- 
processing enzymes (e.g. ZMPSTE24), prelamin of partially processed mutant lamin A (also known as progerin) will accumulate in the NE. Progerin is known to be toxic for the cell, as it interrupts the normal functioning of the nuclear lamina [40-48]. The nuclear envelope will become stiff and rigid, which makes the nucleus more fragile and more vulnerable [49]. This leads to progeria and other progeroid syndromes, in which all tissues are affected and ageing is extremely accelerated.

- Other mutations in the lamin protein can lead to a (partial) loss of lamins. This loss of lamins can lead to a loss of connectivity between the nucleus and the cytoskeleton. The dramatic effects of the loss of the connection between the nucleus and the cytoskeleton on the integrity of the nucleus were demonstrated by Broers et al. [36, 37] and Lammerding et al. [50]. Loss of lamins also can lead to weak spots in the nuclear envelope (blebs, honeycombs and other aberrations), which contain hardly any lamin or associated proteins, thus leaving the nuclear membrane unprotected from external forces.

- This can have a dramatic effect on the nuclear membrane integrity. Many cells demonstrate ruptures of the nuclear membrane, often at weak spots in the nuclear envelope. Because of the ruptures, the compartmentalization between the nucleus and the cytoplasm is temporarily lost, and signalling proteins and transcription factors can diffuse freely in and out of the nucleus. This can have major consequences on the gene regulation in these cells.

The loss of nuclear integrity gave rise to a new hypothesis, which links the structural hypothesis to the gene regulation hypothesis, since it is becomes more and more clear that dysfunctioning of gene regulation can be caused by physical damage to the lamina,

The aim of this thesis was to examine the impact of lamin $\mathrm{A} / \mathrm{C}$ mutations on not only lamina structure, but, most importantly, on nuclear and cellular (dys)functioning. We used mainly human dermal fibroblasts, obtained from patients with different laminopathies. Although this approach is more tedious than studying immortalized and transformed mutant cell line models, 
previous studies have shown that even manipulating wild type LMNA will lead to prominent nuclear changes [36].

We first looked at the loss of structural integrity of the nucleus and of the mechanical network surrounding the nucleus in cells without lamina A/C expression. This was done by examining the structural organizations and integrity of the different components of the nuclear envelope, the LINCcomplex and the different elements of the cytoskeleton (for the latter, not only in the vicinity of the nucleus but also towards the plasma membrane). Also, we have tested whether these mutations lead to an alteration in the mechanical functioning of the cell. This was done by putting cells with mutated lamin under mechanical stress, and observing their behaviour. Alternative methods used to test functionality of the nucleo-cytoskeletal connections were the correct repositioning of the nucleus upon wounding and the reorientation of the MTOC, which is essential for the cell migration process $[51,52]$, and for the formation of membrane protrusions.

Challenged by the abnormalities found in these studies we predicted and increased plasticity of nuclear membrane and the associated chromatin mobility, which indeed could be confirmed in cells without lamin expression.

The observed extreme plasticity of nuclei of LMNA -/- cells urged us to study nuclear membrane behaviour more closely. In some of these cells a sudden drop in intranuclear protein levels was observed. Based on these findings we predicted that nuclear integrity could be compromised in these cells. Indeed, systemically examination of a series of laminopathy cells indicated that in these cells weak spots in the nuclear envelope could lead to dynamic, reversible ruptures of the nuclear membrane at these locations. These ruptures are non-lethal to the cell, and result in a temporary loss of compartmentalization between the nucleus and the cytoplasm, resulting in a redistribution of signalling proteins and transcription factors that normally are actively transported over the nuclear membrane. Nuclear factors that are strongly associated with chromatin will not diffuse out of the nucleus during the ruptures; only the localization of highly dynamic factors can be influenced under these conditions. These dynamic ruptures were seen in a whole variety of laminopathy cell lines. Most strikingly, large, native intranuclear complexes such as PML nuclear bodies were often encountered outside of the nucleus in laminopathy cells. The loss of nuclear integrity caused by these ruptures could well be an alternative mechanism by which the different forms of laminopathies evolve. 


\section{References}

[1] Microsoft Corporation, Encarta ${ }^{\circledR}$ World English Dictionary, Bloomsbury Publishing Plc. 1999.

[2] M. Van Poucke and H. Clijsters, Algemene Biologie: Deel 1.2: De Eukaryote Cel, (1997) p19-20.

[3] D.E. Ingber, Tensegrity I. Cell structure and hierarchical systems biology, J Cell Sci 116 (2003) 1157-1173.

[4] C.J. Hutchison, M. Alvarez-Reyes and O.A. Vaughan, Lamins in disease: why do ubiquitously expressed nuclear envelope proteins give rise to tissue-specific disease phenotypes?, J Cell Sci 114 (2001) 9-19.

[5] J.L. Broers, F.C. Ramaekers, G. Bonne, R.B. Yaou and C.J. Hutchison, Nuclear lamins: laminopathies and their role in premature ageing, Physiol Rev 86 (2006) 967-1008.

[6] E.C. Schirmer, L. Florens, T. Guan, J.R. Yates, 3rd and L. Gerace, Nuclear membrane proteins with potential disease links found by subtractive proteomics, Science 301 (2003) 1380-1382.

[7] Q. Zhang, J.N. Skepper, F. Yang, J.D. Davies, L. Hegyi, R.G. Roberts, P.L. Weissberg, J.A. Ellis and C.M. Shanahan, Nesprins: a novel family of spectrin-repeat-containing proteins that localize to the nuclear membrane in multiple tissues, J Cell Sci 114 (2001) 4485-4498.

[8] Y.Y. Zhen, T. Libotte, M. Munck, A.A. Noegel and E. Korenbaum, NUANCE, a giant protein connecting the nucleus and actin cytoskeleton, J Cell Sci 115 (2002) 3207-3222.

[9] K. Wilhelmsen, S.H. Litjens, I. Kuikman, N. Tshimbalanga, H. Janssen, I. van den Bout, K. Raymond and A. Sonnenberg, Nesprin3 , a novel outer nuclear membrane protein, associates with the cytoskeletal linker protein plectin, J Cell Biol 171 (2005) 799-810.

[10] K.J. Roux, M.L. Crisp, Q. Liu, D. Kim, S. Kozlov, C.L. Stewart and B. Burke, Nesprin 4 is an outer nuclear membrane protein that can induce kinesin-mediated cell polarization, Proc Natl Acad Sci U S A 106 (2009) 2194-2199.

[11] M. Crisp, Q. Liu, K. Roux, J.B. Rattner, C. Shanahan, B. Burke, P.D. Stahl and D. Hodzic, Coupling of the nucleus and cytoplasm: role of the LINC complex, J Cell Biol 172 (2006) 41-53. 
[12] D.E. Ingber, Tensegrity II. How structural networks influence cellular information processing networks, J Cell Sci 116 (2003) 1397-1408.

[13] D.E. Ingber, Cellular mechanotransduction: putting all the pieces together again, Faseb J 20 (2006) 811-827.

[14] D.E. Ingber, Tensegrity and mechanotransduction, J Bodyw Mov Ther 12 (2008) 198-200.

[15] M. Caron, M. Auclair, C. Vigouroux, M. Glorian, C. Forest and J. Capeau, The HIV protease inhibitor indinavir impairs sterol regulatory element-binding protein-1 intranuclear localization, inhibits preadipocyte differentiation, and induces insulin resistance, Diabetes 50 (2001) 1378-1388.

[16] C. Capanni, E. Mattioli, M. Columbaro, E. Lucarelli, V.K. Parnaik, G. Novelli, M. Wehnert, V. Cenni, N.M. Maraldi, S. Squarzoni and G. Lattanzi, Altered pre-lamin A processing is a common mechanism leading to lipodystrophy, Hum Mol Genet 14 (2005) 1489-1502.

[17] V. Nikolova, C. Leimena, A.C. McMahon, J.C. Tan, S. Chandar, D. Jogia, S.H. Kesteven, J. Michalicek, R. Otway, F. Verheyen, S. Rainer, C.L. Stewart, D. Martin, M.P. Feneley and D. Fatkin, Defects in nuclear structure and function promote dilated cardiomyopathy in lamin A/C-deficient mice, J Clin Invest 113 (2004) 357-369.

[18] D.J. Lloyd, R.C. Trembath and S. Shackleton, A novel interaction between lamin A and SREBP1: implications for partial lipodystrophy and other laminopathies, Hum Mol Genet 11 (2002) 769-777.

[19] J.H. Van Berlo, J.W. Voncken, N. Kubben, J.L. Broers, R. Duisters, R.E. van Leeuwen, H.J. Crijns, F.C. Ramaekers, C.J. Hutchison and Y.M. Pinto, A-type lamins are essential for TGF-beta1 induced PP2A to dephosphorylate transcription factors, Hum Mol Genet 14 (2005) 2839-2849.

[20] B.R. Johnson, R.T. Nitta, R.L. Frock, L. Mounkes, D.A. Barbie, C.L. Stewart, E. Harlow and B.K. Kennedy, A-type lamins regulate retinoblastoma protein function by promoting subnuclear localization and preventing proteasomal degradation, Proc Natl Acad Sci U S A 101 (2004) 9677-9682.

[21] A. Muchir, J. Shan, G. Bonne, S.E. Lehnart and H.J. Worman, Inhibition of extracellular signal-regulated kinase signaling to prevent cardiomyopathy caused by mutation in the gene encoding Atype lamins, Hum Mol Genet 18 (2009) 241-247. 
[22] A. Muchir, W. Wu and H.J. Worman, Reduced expression of A-type lamins and emerin activates extracellular signal-regulated kinase in cultured cells, Biochim Biophys Acta 1792 (2009) 75-81.

[23] V. Andres and J.M. Gonzalez, Role of A-type lamins in signaling, transcription, and chromatin organization, J Cell Biol 187 (2009) 945-957.

[24] A. Nagano, R. Koga, M. Ogawa, Y. Kurano, J. Kawada, R. Okada, Y.K. Hayashi, T. Tsukahara and K. Arahata, Emerin deficiency at the nuclear membrane in patients with Emery-Dreifuss muscular dystrophy, Nat Genet 12 (1996) 254-259.

[25] B. Burke, L.C. Mounkes and C.L. Stewart, The nuclear envelope in muscular dystrophy and cardiovascular diseases, Traffic 2 (2001) 675-683.

[26] R. Somech, S. Shaklai, N. Amariglio, G. Rechavi and A.J. Simon, Nuclear envelopathies--raising the nuclear veil, Pediatr Res 57 (2005) 8R-15R.

[27] G. Bonne, R.B. Yaou, C. Beroud, G. Boriani, S. Brown, M. de Visser, D. Duboc, J. Ellis, I. Hausmanowa-Petrusewicz, G. Lattanzi, L. Merlini, G. Morris, F. Muntoni, G. Opolski, Y.M. Pinto, F. Sangiuolo, D. Toniolo, R. Trembath, J.H. van Berlo, A.J. van der Kooi and M. Wehnert, 108th ENMC International Workshop, 3rd Workshop of the MYO-CLUSTER project: EUROMEN, 7th International Emery-Dreifuss Muscular Dystrophy (EDMD) Workshop, 13-15 September 2002, Naarden, The Netherlands, Neuromuscul Disord 13 (2003) 508-515.

[28] B. Burke and C.L. Stewart, Life at the edge: the nuclear envelope and human disease, Nat Rev Mol Cell Biol 3 (2002) 575-585.

[29] Y. Gruenbaum, A. Margalit, R.D. Goldman, D.K. Shumaker and K.L. Wilson, The nuclear lamina comes of age, Nat Rev Mol Cell Biol 6 (2005) 21-31.

[30] C.J. Hutchison and H.J. Worman, A-type lamins: guardians of the soma?, Nat Cell Biol 6 (2004) 1062-1067.

[31] J.L. Broers, C.J. Hutchison and F.C. Ramaekers, Laminopathies, J Pathol 204 (2004) 478-488.

[32] H.J. Worman, L.G. Fong, A. Muchir and S.G. Young, Laminopathies and the long strange trip from basic cell biology to therapy, J Clin Invest 119 (2009) 1825-1836.

[33] H.J. Worman, C. Ostlund and Y. Wang, Diseases of the nuclear envelope, Cold Spring Harb Perspect Biol 2 (2010) a000760. 
[34] M. Cohen, K.K. Lee, K.L. Wilson and Y. Gruenbaum, Transcriptional repression, apoptosis, human disease and the functional evolution of the nuclear lamina, Trends Biochem Sci 26 (2001) 41-47.

[35] N.M. Maraldi, C. Capanni, V. Cenni, M. Fini and G. Lattanzi, Laminopathies and lamin-associated signaling pathways, J Cell Biochem 112 (2011) 979-992.

[36] J.L. Broers, E.A. Peeters, H.J. Kuijpers, J. Endert, C.V. Bouten, C.W. Oomens, F.P. Baaijens and F.C. Ramaekers, Decreased mechanical stiffness in LMNA-/- cells is caused by defective nucleo-cytoskeletal integrity: implications for the development of laminopathies, Hum Mol Genet 13 (2004) 2567-2580.

[37] F. Houben, F.C. Ramaekers, L.H. Snoeckx and J.L. Broers, Role of nuclear lamina-cytoskeleton interactions in the maintenance of cellular strength, Biochim Biophys Acta 1773 (2007) 675-686.

[38] F. Houben, C.H. Willems, I.L. Declercq, K. Hochstenbach, M.A. Kamps, L.H. Snoeckx, F.C. Ramaekers and J.L. Broers, Disturbed nuclear orientation and cellular migration in A-type lamin deficient cells, Biochim Biophys Acta 1793 (2009) 312-324.

[39] M. Schneider, W. Lu, S. Neumann, A. Brachner, J. Gotzmann, A.A. Noegel and I. Karakesisoglou, Molecular mechanisms of centrosome and cytoskeleton anchorage at the nuclear envelope, Cell Mol Life Sci (2011).

[40] M.O. Bergo, B. Gavino, J. Ross, W.K. Schmidt, C. Hong, L.V. Kendall, A. Mohr, M. Meta, H. Genant, Y. Jiang, E.R. Wisner, N. Van Bruggen, R.A. Carano, S. Michaelis, S.M. Griffey and S.G. Young, Zmpste24 deficiency in mice causes spontaneous bone fractures, muscle weakness, and a prelamin A processing defect, Proc Natl Acad Sci U S A 99 (2002) 13049-13054.

[41] A.M. Pendas, Z. Zhou, J. Cadinanos, J.M. Freije, J. Wang, K. Hultenby, A. Astudillo, A. Wernerson, F. Rodriguez, K. Tryggvason and C. Lopez-Otin, Defective prelamin A processing and muscular and adipocyte alterations in Zmpste24 metalloproteinase-deficient mice, Nat Genet 31 (2002) 94-99.

[42] M. Eriksson, W.T. Brown, L.B. Gordon, M.W. Glynn, J. Singer, L. Scott, M.R. Erdos, C.M. Robbins, T.Y. Moses, P. Berglund, A. Dutra, E. Pak, S. Durkin, A.B. Csoka, M. Boehnke, T.W. Glover and F.S. Collins, Recurrent de novo point mutations in lamin A cause Hutchinson-Gilford progeria syndrome, Nature 423 (2003) 293-298. 
[43] L.G. Fong, J.K. Ng, M. Meta, N. Cote, S.H. Yang, C.L. Stewart, T. Sullivan, A. Burghardt, S. Majumdar, K. Reue, M.O. Bergo and S.G. Young, Heterozygosity for Lmna deficiency eliminates the progerialike phenotypes in Zmpste24-deficient mice, Proc Natl Acad Sci U S A 101 (2004) 18111-18116.

[44] C.L. Navarro, A. De Sandre-Giovannoli, R. Bernard, I. Boccaccio, A. Boyer, D. Genevieve, S. Hadj-Rabia, C. Gaudy-Marqueste, H.S. Smitt, P. Vabres, L. Faivre, A. Verloes, T. Van Essen, E. Flori, R. Hennekam, F.A. Beemer, N. Laurent, M. Le Merrer, P. Cau and N. Levy, Lamin A and ZMPSTE24 (FACE-1) defects cause nuclear disorganization and identify restrictive dermopathy as a lethal neonatal laminopathy, Hum Mol Genet 13 (2004) 2493-2503.

[45] J. Gruber, T. Lampe, M. Osborn and K. Weber, RNAi of FACE1 protease results in growth inhibition of human cells expressing lamin A: implications for Hutchinson-Gilford progeria syndrome, J Cell Sci 118 (2005) 689-696.

[46] S. Shackleton, D.T. Smallwood, P. Clayton, L.C. Wilson, A.K. Agarwal, A. Garg and R.C. Trembath, Compound heterozygous ZMPSTE24 mutations reduce prelamin A processing and result in a severe progeroid phenotype, J Med Genet 42 (2005) e36.

[47] S.G. Young, L.G. Fong and S. Michaelis, Prelamin A, Zmpste24, misshapen cell nuclei, and progeria--new evidence suggesting that protein farnesylation could be important for disease pathogenesis, J Lipid Res 46 (2005) 2531-2558.

[48] D. McClintock, L.B. Gordon and K. Djabali, Hutchinson-Gilford progeria mutant lamin A primarily targets human vascular cells as detected by an anti-Lamin A G608G antibody, Proc Natl Acad Sci U S A 103 (2006) 2154-2159.

[49] V.L. Verstraeten, J. Renes, F.C. Ramaekers, M. Kamps, H.J. Kuijpers, F. Verheyen, M. Wabitsch, P.M. Steijlen, M.A. van Steensel and J.L. Broers, Reorganization of the nuclear lamina and cytoskeleton in adipogenesis, Histochem Cell Biol 135 (2011) 251261.

[50] J. Lammerding, P.C. Schulze, T. Takahashi, S. Kozlov, T. Sullivan, R.D. Kamm, C.L. Stewart and R.T. Lee, Lamin A/C deficiency causes defective nuclear mechanics and mechanotransduction, J Clin Invest 113 (2004) 370-378.

[51] J.S. Lee, C.M. Hale, P. Panorchan, S.B. Khatau, J.P. George, Y. Tseng, C.L. Stewart, D. Hodzic and D. Wirtz, Nuclear lamin A/C 
deficiency induces defects in cell mechanics, polarization, and migration, Biophys J 93 (2007) 2542-2552.

[52] M. Schneider, W. Lu, S. Neumann, A. Brachner, J. Gotzmann, A.A. Noegel and I. Karakesisoglou, Molecular mechanisms of centrosome and cytoskeleton anchorage at the nuclear envelope, Cell Mol Life Sci. 


\section{Chapter 1}

\section{Role of nuclear lamina- cytoskeleton interactions in the maintenance of cellular strength}

F. Houben, F.C.S. Ramaekers, L.H.E.H. Snoeckx, J.L.V. Broers

Published in part in:

Biochimica Biophysica Acta - Molecular Cell Research; 1773 (2007) 675-686 


\section{Abstract}

The response of individual cells to cellular stress is vital for cellular functioning. A large network of physically interconnected cellular components, starting from the structural components of the cells' nucleus, via cytoskeleton filaments to adhesion molecules and the extracellular matrix, constitutes an integrated matrix that functions as a scaffold allowing the cell to cope with mechanical stress. Next to a role in mechanical properties, this network also has a mechanotransductional function in the response to mechanical stress. This signaling route does not only regulate a rapid reorganization of structural components such as actin filaments, but also stimulates for example gene activation via NFאB and other transcription factors. The importance of an intact mechano-signaling network is illustrated by the physiological consequences of several genetic defects of cellular network components e.g. actin, dystrophin, desmin and lamins. These give rise to an impaired response of the affected cells to mechanical stress and often result in dystrophy of the affected tissue. Recently, the importance of the cell nucleus in cellular strength has been established. Several new interconnecting proteins, such as the nesprins that link the nuclear lamina to the cytoskeleton, have been identified. Furthermore, the function of nuclear lamins in determining cellular strength and nuclear stability was illustrated in lamin-knock-out cells. Absence of the A-type lamins or mutations in these structural components of the nuclear lamina leads to an impaired cellular response to mechanical stress and disturbances in cytoskeletal organization. In addition, laminopathies show clinical phenotypes comparable to those seen for diseases resulting from genetic defects in cytoskeletal components, further indicating that lamins play a central role in maintaining the mechanical properties of the cell. 


\section{Introduction}

Cells are flexible building blocks of tissues that interact with other cells in a dynamic fashion. These systems can be continuously subjected to various forms of mechanical stress, such as deformation by compression and stretching. While stress on muscle cells is obvious, also surrounding cells, such as muscle fibroblasts, experience constant stretching and compression forces during muscular activity. Cells must be able to cope with this stress to survive and exert their function in a normal manner. It has become clear that animal cells exhibit a considerable mechanical rigidity (stiffness) through their cytoskeleton $[1,2]$. One of the theories explaining this stiffness is cellular tensegrity, a structural model for the mechanical properties of the living cell. In this model the cell is designed as a prestressed tensegrity structure [1-6], in which the cytoskeleton forms an internal scaffold that can absorb the mechanical loading of the cell. In the tensegrity model, tensional forces originate from microfilaments and compressional forces are exerted on microtubules $[1,2,7]$. In this model, the cytoskeleton is not static but flexible and adjustable so that it can adapt to variations in direction and the degree of mechanical stress. The interconnected structures of the cytoskeleton form equilibrium, a balance of internal forces of tension and compression. This structure thus functions as a scaffold that can resist external mechanical forces [8]. It is generally suggested that the microfilaments function as tension elements that will make the cell more stiff and rigid in response to stretch. In contrast, microtubules function as compression elements, stiffening the cell during compression [1, 2]. For optimal force transmission towards the cells' nucleus, prestress in the intact cytoskeleton cell is a prerequisite [9].

However, not only the cytoskeleton plays an important role in the response to mechanical stress. Cells interact with each other and with the extracellular matrix through cell adhesion proteins, thereby transmitting stress onto neighbouring cells throughout the tissue, and within the cell from the cellular membrane towards the nucleus. Next to their mechanical role, cell adhesion molecules transduce stress signals, thus functioning as mechanoreceptors [10]. This mechanotransduction mechanism can induce a whole series of cellular responses, such as formation of stress fibers and activation of genes for stress proteins, which will help the cell to resist the mechanical loading [11].

Recent studies indicate that also the structural integrity of the nucleus plays an important role in the resistance against mechanical stress. Next to the nuclear membrane, the nuclear lamina, and the lamina-associated proteins seem to be 
determining factors for resistance to stress. This can be derived from the fact that different mutations in the genes for lamina proteins such as A- and B-type lamins, and emerin are associated with a series of pathologies, collectively named the laminopathies (for a recent review, see [12]), that are amongst others characterized by a reduced resilience to mechanical stress. Next to the mechanical disruptions, also changes in the signaling pathways and the regulation of gene expression can be observed in these pathologies. This review focuses on the interactions between the nuclear lamina and the cytoskeleton, and its subsequent influence on cellular adhesion.

\section{Mechanical strength of cells}

\section{The nuclear lamina and intranuclear structures}

Lamins are type $\mathrm{V}$ intermediate filament proteins that associate with the inner nuclear membrane and form the meshwork of the nuclear lamina [13]. Lamin proteins consist of a central helical rod domain and a globular head and tail domains. A nuclear localization signal (NLS) in the tail domain of the protein directs the whole protein towards the inner nuclear membrane.

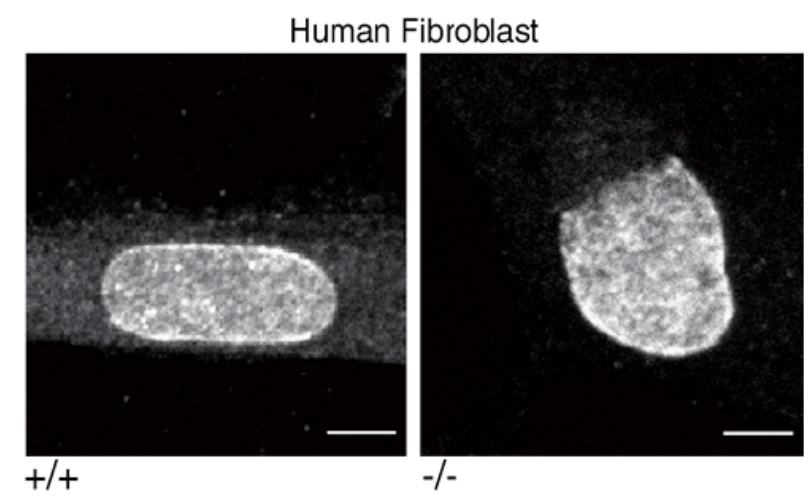

Fig. 1. Distribution of Lamin B1 in wild type (+/+) and Lamin A/C deficient (-/-) human fibroblasts. Note partial absence of lamin B1 in an area of the nucleus. All scale bars are $10 \mu \mathrm{m}$

Two types of lamins exist in eukaryotic cells, i.e. the A-type lamins (lamins $\mathrm{A}, \mathrm{A} \Delta 10$, $\mathrm{C}$ and $\mathrm{C} 2$ ), and the B-type lamins (B1, B2 and B3) [14-20], which differ in their expression patterns. While A-type lamins are expressed in several cell types upon differentiation [21-24], B-type lamins are expressed in virtually all cell types, both 
embryonic and adult $[21,23,25]$. This implies that the role of the B-type lamins in the cell seems to be vital, which can also be concluded from the fact that mutations in the lamin-B gene (LMNB) are not compatible with life [16, 26]. Mutations in the lamin-A gene (LMNA) are associated with several types of laminopathies [27].

Mouse Embryonic Fibroblast

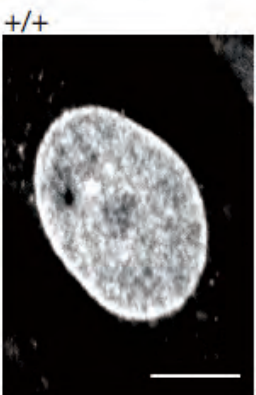

Emerin

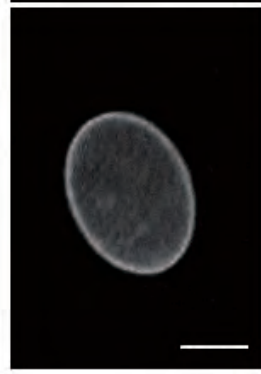

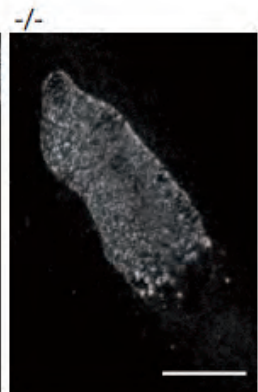

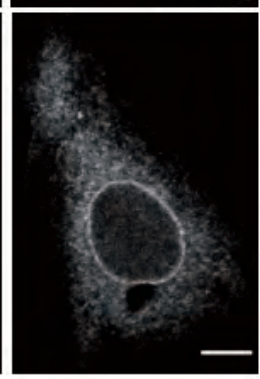

Human Fibroblast
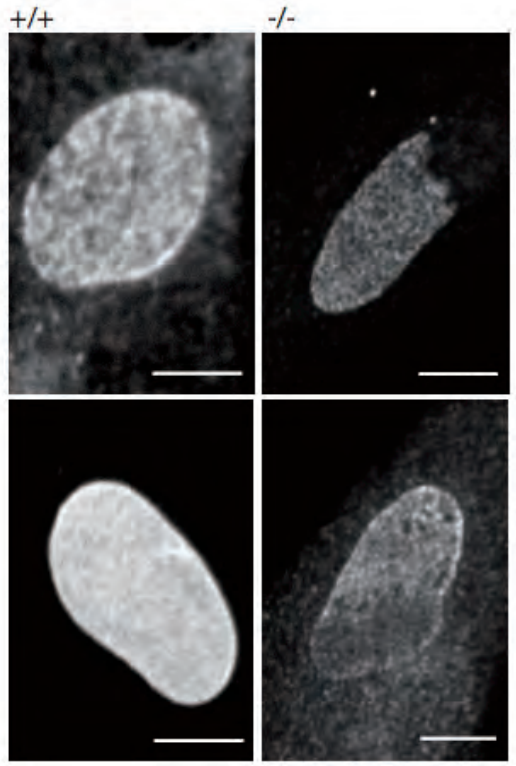

Fig. 2. Distribution of lamin-associated nucleoporin (p62) and emerin in wild type (+/+) and lamin A/C deficient $(-/-)$ mouse and human fibroblasts Note absence of p62 in large areas of the nucleus in A-type lamin deficient cells. In these cells emerin is localized to the endoplasmic reticulum. All scale bars are 10 $\mu \mathrm{m}$.

The expression patterns and importance for cell survival of these two main lamin subtypes support the assumption that B-type lamins are the fundamental building blocks of the nuclear lamina, while A-type lamins have more specialized functions [28]. The lamina is essential for the maintenance of nuclear shape and size [20] and for correct intra-nuclear mechanotransduction [29-31]. Because the nuclear structure accounts for most of the mechanical stiffness of a cell $[32,33]$, the maintenance of structural integrity of the nucleus will be an important factor for the cells' capacity to endure mechanical stress.

The lamina network generates a certain nuclear stiffness [31, 34], because several proteins like nuclear actin and emerin stabilize this network and ensure an adequate response to compression and mechanical stress [35-37]. 
Lamins are also present in the nucleoplasm [38, 39], nuclear bodies [40] and in tubular invaginations of the nuclear envelope [41-45]. The exact function of these structures remains unclear, but they may play a regulatory role in DNA-replication and DNAtranscription, or in structural support.

The connection between nuclear actin and protein 4.1 (which can link microfilaments and short actin strands to membranes) [46-49], with lamins A, B and C, and emerin, indicates that nuclear actin is likely to participate in the organization of the nucleoskeleton, in the maintenance of cellular integrity and the response to mechanical injury [36, 37]. The lamin proteins are also important for the correct distribution of other lamina proteins (see Fig. 1), as well as several nuclear membrane associated proteins [27] (see Fig. 2).

\section{The nuclear membrane and associated proteins}

At the nuclear membrane, several proteins form connections with the underlying lamina structure on the one hand or the cytoskeleton on the other. These proteins are called Nuclear Envelope Transmembrane (NET) proteins. Amongst these are several that are likely to participate in the mechanical integrity of the cell and nucleus:

(A) Lamina Associated Polypeptides (LAP) are a superfamily of type II integral membrane protein [50]. The LAP2 family, with six isoforms, is best characterized. Members of it bind primarily to B-type lamins which is critical for the survival of the cell $[51,52]$. One isoform (LAP2 $\alpha$ ) lacks a transmembrane domain and binds to A-type lamins in the nucleoplasm [53, 54].

(B) Emerin is a type II integral membrane protein that can bind to all types of lamins [55-57]. It helps to maintain the mechanical integrity of the nucleus, since mutations in the emerin gene induce impaired mechanotransduction [58], leading to EmeryDreifuss muscular dystrophy [59, 60], one of the known laminopathies.

(C) MAN-1 is an essential protein for early development, which binds to lamins in a manner similar to emerin [61]. The binding of these 3 proteins i.e. LAP2, emerin and MAN, to the lamin proteins occurs through a $\sim 40$ residue conserved LEM domain [62].

(D) The Lamin B Receptor (LBR) is also located at the inner nuclear membrane. LBR is essential for fetal development [63], although it is as yet unclear whether this is through its function as a sterol reductase or through its putative role in anchoring chromatin to the nuclear envelope [64].

The described NET proteins are transmembrane proteins in the inner nuclear membrane, which bind to the lamin filaments and thus form part of the nuclear lamina [65]. Through their binding with lamins, these proteins are properly organized and 
retained in the inner nuclear membrane [66]. In cells lacking A-type lamins, several proteins associated with the nuclear membrane (like emerin and P62 nucleoporin) are mislocalized, indicating that their connection with lamin $\mathrm{A} / \mathrm{C}$ is necessary for the correct distribution of these proteins [27] (see Fig. 2). Next to the connection between the inner nuclear membrane and the nuclear lamina, the nucleus is also connected to the cytoskeletal filaments through linker proteins. While the existence of these linkerproteins has long been suggested, their exact nature and structure are still matter of discussion. The recently discovered Nesprin and SUN proteins are good candidates for the link between the nuclear lamina and nuclear membrane on the one hand and the cytoskeleton on the other hand.

(E) Nesprins are high molecular weight proteins associated with the nuclear membrane $[67,68]$. Four different types are currently described: Syne-1/Myne-1/Nesprin1/Enaptin and Syne-2/Myne-2/Nesprin-2/NUANCE [67, 69-71], Nesprin-3 [72] and Nesprin-4 [73]. Nesprin isoforms associate with the inner or outer nuclear membrane through their transmembrane KASH-domain (Klarsicht/ANC-1/Syne-1 homology) at the carboxy-terminus $[68,74,75]$. The KASH-domain consists of a transmembrane region that is essential for the localization of nesprin to the nuclear membrane, and a small (less then 35 residues) luminal region that is located in the perinuclear space [74]. Nesprins can translocate from the outer to the inner nuclear membrane through the nuclear pore membrane, but there is a restriction in size. The larger isoforms of nesprin-1 and -2 cannot translocate into the nucleus and are therefore only found in the outer nuclear membrane [76]. When nesprins are associated with the outer nuclear membrane, the amino-terminus is exposed towards the cytoplasm and binds to microfilaments [68, 70], intermediate filaments [72] and microtubules [73]. As such nesprins connect the nucleus to the cytoskeleton $[6,75]$ and consequently to adhesion complexes at the plasma membrane [77]. This anchorage of the nuclear membrane to the cytoskeleton is essential for migration and correct localization of the nucleus inside the cell. Nesprins at the inner nuclear membrane (smaller isoforms) bind to lamin $\mathrm{A} / \mathrm{C}$ and emerin $[78,79]$ through their spectrin repeats in the carboxy-terminus, and - as such - interact closely with the nuclear lamina.

(F) SUN-proteins are the second group of proteins that are part of the complex linking the nucleus to the cytoskeleton $[75,80]$. They are associated with the inner nuclear membrane, with their carboxy-terminus in the perinuclear space, and their aminoterminus in the nuclear interior. The carboxy-terminus contains the evolutionary conserved SUN-domain (Sad1/UNC-84 homologue) [79, 81, 82], which in C. elegans forms the connection between ANC and UNC-84 [83]. However in human cells, this SUN-domain is not important for the binding of the KASH-domain of nesprin-2 to the SUN-proteins. Instead, this is mediated by the so-called SD2 domain of the SUN1 
protein [79]. Which domains are needed to connect the other human isoforms of nesprins and SUN-proteins, remains to be discovered. SUN proteins are necessary for the correct localization of nesprins to the nuclear envelope [79], and can directly bind to lamins $\mathrm{A} / \mathrm{C}$. The whole complex, formed by nesprins and SUN proteins, is called the LINC-complex (LInker of the Nucleoskeleton and the Cytoskeleton), and connects the nuclear envelope with both the nucleoskeleton and the cytoskeleton [84]. The interaction of the KASH-domain of nesprins and the SUN proteins is fundamental for the formation of this complex [84].

Next to the LINC complex proteins, other nuclear envelope proteins can mediate the connection between the nucleus and the cytoskeleton. For instance, recently, the protein connecting the nuclear lamina to the MTOC was discovered, called SAMP1 $[85,86]$. This protein associates both with lamins, emerin, and SUN1. Most likely many other NE proteins remain to be examined four their function. In rat liver it is estimated that at least 80 different NE proteins can be detected [87]

The importance of the link between the nucleus and the cytoskeleton for the cellular response to mechanical loading becomes evident using a single cell compression device [30]. When subjected to compressive forces, nuclei of wild-types cells deform in a anisotropic manner, i.e. perpendicular to the growth direction of the cell [88]. This typical behaviour is most likely caused by nucleus-actin interactions.

In contrast, nuclei of A-type lamin-deficient cells deform isotropically, i.e. upon compression they show equal expansion of the nucleus into all directions (Fig. 3), indicating the loss of connectivity between the nucleus and the cytoskeleton.

If nuclei are physically disconnected from the cytoskeleton and isolated from the whole cell by mechanical force, they all deform isotropically, whether the nuclei are lamindeficient or not. These findings indicate that the nucleus in a normal cell is embedded in the cytoskeletal structure surrounding the nucleus, forming strong connections and thus forming one entity that responds to external mechanical forces and protects the nucleus against mechanical forces (see Fig. 3). 


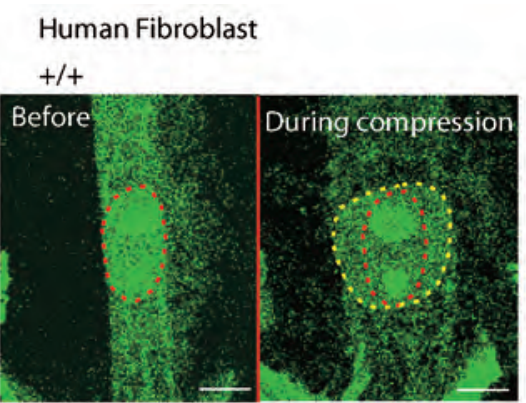

$-$

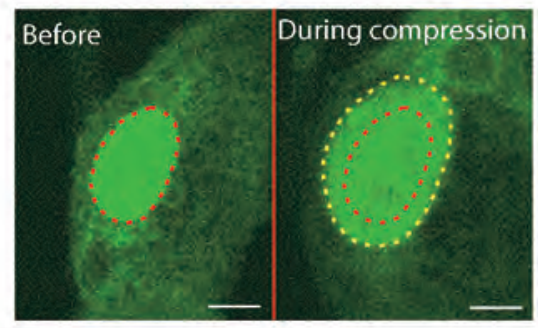

Isolated Nuclei of Mouse Embryonic Fibroblast

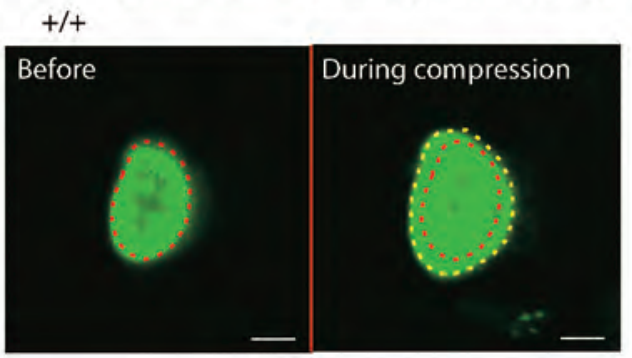

$-1-$

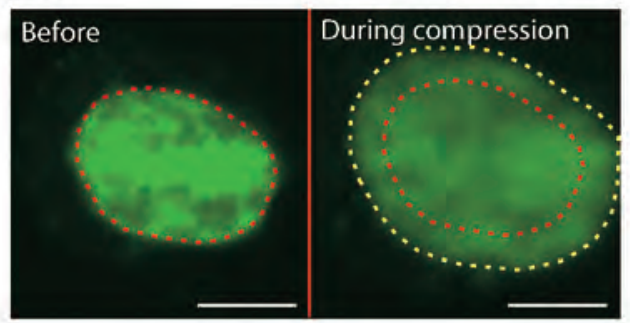

Fig. 3. Compression of wild-type $(+/+)$ or A-type lamin deficient $(-/-)$ human fibroblasts and isolated nuclei of Mouse Embryonic Fibroblasts. Nuclei of fibroblasts with a normal lamina (+/+) deform anisotropically, while nuclei of fibroblasts with Lamin A/C deficiency deform isotropically. Isolated nuclei, that are disconnected from the cytoskeleton, deform isotropically. All scale bars are $10 \mu \mathrm{m}$.

\section{The cytoskeleton}

In the tensegrity model, the filaments of the cytoskeleton form the most important part of the prestressed mechanical structure in the cell. They are connected to the plasma membrane through the cell adhesion molecules, and to the nucleus through the nesprinand SUN-proteins (Fig. 4).

In comparison to microtubuli, the microfilaments can cope with a relatively high degree of mechanical stretch with only a mild deformation, but like microtubuli they do rupture easily [89]. Actin filaments provide structural support to the cellular plasma membrane, due to their interaction with proteins in the plasma membrane. Furthermore, microfilaments link to elements of the extracellular matrix. When cells experience new conditions and external stress, actin can be repolymerized into new structures in order to cope with these changes. For example, a cell under mechanical stress will form stress fibers that predominantly contain actin [90]. 


\section{Mouse Embryonic Fibroblast}

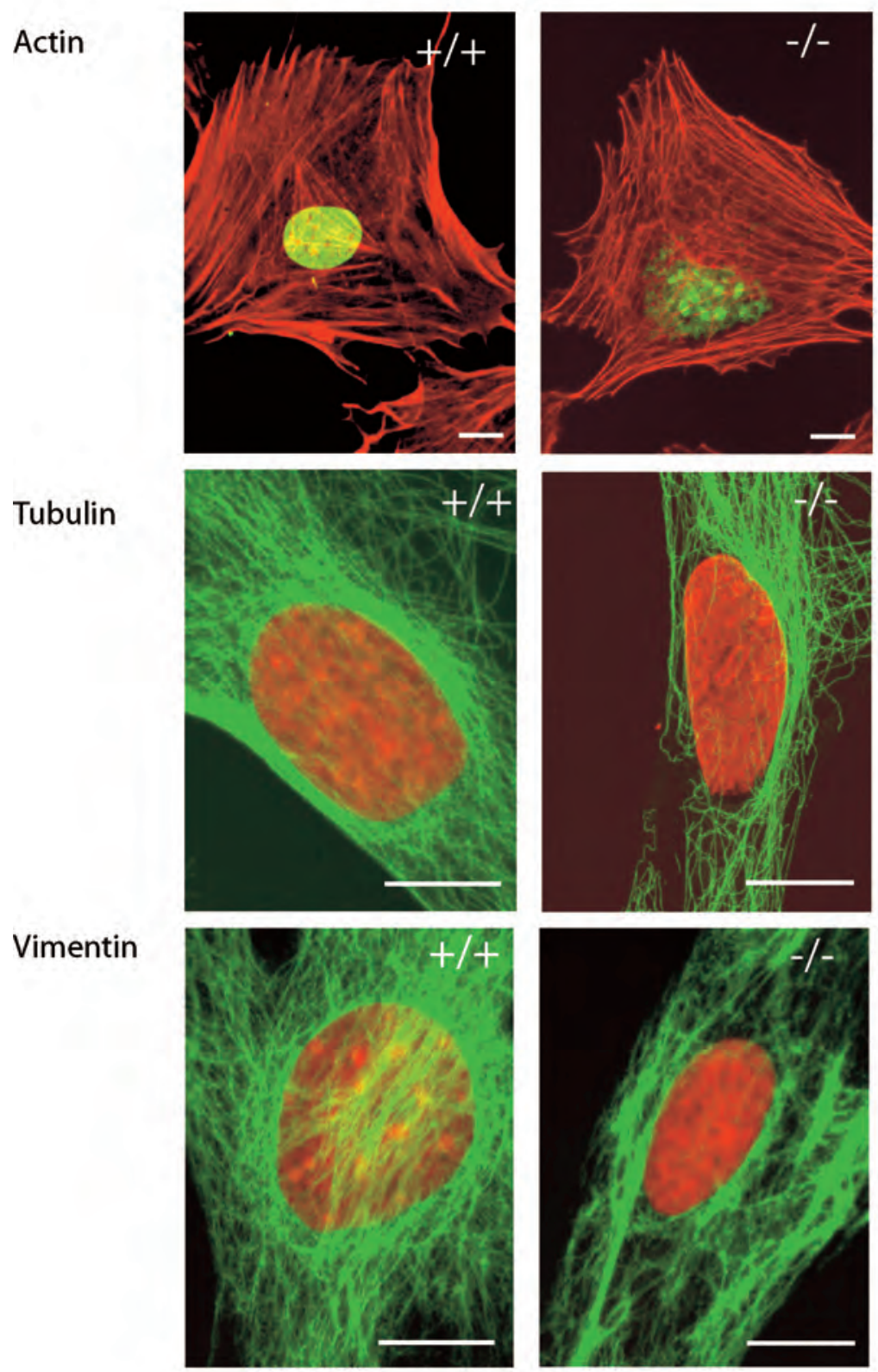

Fig. 4. Alterations in the cytoskeletal structures in Lamin A/C deficient (-/-) mouse fibroblasts, compared to wild-type $(+/+)$ fibroblasts. Note irregularities in actin, tubulin and intermediate filament networks in $-/-$ cells. All scale bars are $10 \mu \mathrm{m}$. 
These stress fibers help in maintaining the cell shape and a certain level of cellular stiffness during the mechanical loading [89, 91, 92]. Rearrangement of these stress fibers is regulated by several signaling proteins (see below).

The stiffness of the actin filaments can vary by small changes in cross-linking between the individual filaments [93] and in actin-associated proteins [94, 95]. Next to the structural role of actin, several mechanosensory and mechanotransduction pathways can be activated by mechanical stretch of the fibers [96].

Both the actin filaments and the actin-associated proteins can act as sensors for stretch or compression [97] (see below). Microtubules play an essential role in the internal cellular organization and localization of the cell organelles [98] such as mitochondria and the nucleus. They function as a scaffold for internal transport of vesicles and cell organelles [99-102]. Motor proteins like kinesin, dynein [103] and Hook3 [104] connect to vesicles and move these in both directions along the microtubular network. During mitosis, the microtubules play an essential role in the formation of mitotic spindles, crucial for dividing the chromosomes over de daughter cells [105].

In comparison to microfilaments and intermediate filaments, microtubules are more fragile when subjected to repeated compression and stretching [89, 106, 107]. Microtubules regulate the response of the cell to mechanical stress. As the actin network provides the pre-stress balance, a disturbance of this balance due to an external mechanical stress, can affect the microtubular structure and thus change the stiffness of the cells. This reorganization can soften or harden microtubules depending on the extent of the cell distension [107-110]. In contrast to the other cytoskeletal proteins, intermediate filaments can withstand larger mechanical stress without rupturing [89], making them an essential factor in maintaining cellular integrity [89].

Within the cytoskeleton, they interact with the other cytoskeletal elements and mediate signaling events, interacting with several protein kinases [111-113]. The linker protein plectin plays an essential role in linking intermediate filaments to each other, to the other elements of the cytoskeleton $[114,115]$, to the nuclear membrane (through nesprin) [72] and to the adhesion complexes in the plasma membrane [116].

The cytoplasmic intermediate filaments are essential for the correct mechanical functioning of the cell, which has become obvious from studies in patients with genetic defects affecting intermediate filaments [117-120].

Next to the cytoplasmic intermediate filaments, the nuclear intermediate filaments consisting of lamins play an important role in the mechanical behavior of nuclei and cells, as mentioned above. 


\section{Cell adhesion molecules and the extracellular matrix}

Cell adhesion molecules are located at the cellular plasma membrane and are part of the mechanically balanced system of the cell and tissue. Next to their mechanical role, cell adhesion molecules play an important role in 'sensing' mechanical stress and in transducing stress signals towards the cytoskeleton and the nucleus (mechanotransduction). Cell-cell adhesion molecules include the connexins and cadherins, while cell-matrix interactions, which are critical for cellular adhesion and force transduction towards the extracellular matrix, involve the integrins.

Connexins are connected to the cytoskeleton through connective proteins such as drebin [121], suggesting that the formation and the maintenance of connexons depend on a well functioning cytoskeleton. On the other hand connexins help maintain the structure of the cytoskeleton by linking it to the plasma membrane [121-123].

Cadherins are calcium-dependent plasma membrane-associated cell adhesion molecules that can be divided into 4 main groups, the E-cadherins (epithelia), the Pcadherins (placenta, epidermis), the VE-cadherins (endothelial cells) and the NCadherins (nervous tissue, cardiac and skeletal muscle tissue, fibroblasts) [124]. Cadherins are connected to the actin cytoskeleton through catenin proteins and have a designated role in the mechanotransduction through the activation of stretch-sensitive calcium channels and the transduction of forces onto the cytoskeleton $[125,126]$. Interaction of cells with the extracellular matrix is mediated through integrin molecules [127-130]. These consist of two transmembrane glycoprotein subunits, the $\alpha$ - and $\beta$ subunits [131], for which many isoforms are described. Integrins connect the cytoskeleton to the extracellular matrix such that mechanical stress can be conferred from the cytoskeleton to the extracellular matrix, and vice versa from the extracellular matrix to the cytoskeleton $[4,132]$. The connection of the integrins with the different elements of the extracellular matrix (e.g. collagen, laminin, fibronectin) is specific for different combinations of $\alpha$ - and $\beta$-subunits. Integrins are integrated in signaling pathways [133], in such a way that they can transduce the stress signal in a biochemical and in a mechanical way.

\section{Mechanical properties of the cell}

As described above, cells contain one interconnected scaffolding network connecting the structural proteins in the nucleus via the nuclear membrane to the cytoskeleton, cell adhesion molecules and the extracellular matrix (see Fig. 5).

The integrity of such a complex network is essential for the transmission of mechanically induced signals from the plasma membrane and adhesion molecules via 
the cytoskeleton back to the nuclear matrix (see Fig. 6). It thus generates an appropriate nuclear response to cope with the mechanical stress. This response can in turn induce changes in the cytoskeleton and in the adhesion complexes. As such not only the cytoplasm but the whole cellular structure functions as the pre-stressed scaffold, as described in the tensegrity model [1,2]. All the individual elements form one interacting mechanical entity that cannot function properly if one of the elements is deleted or becomes disfunctional. This can for example be appreciated in the genetic disorders affecting the cytoskeletal structures. Mutations of elements of the cytoskeleton such as actin filaments $[134,135]$ and intermediate filaments $[27,117-$ 120, 136], mutations of cell adhesion proteins [137-139] and of linker proteins such as plectin [140] and dystrophin [141, 142] all give rise to comparable clinical features [143] i.e. dystrophy of the affected tissue and a decreased mechanical strength of the cell. Also, mutations in the nuclear lamina structure give rise to alterations in the cytoskeleton [30] (see Fig. 4).

Next to the pure mechanical properties of this complex, there is also biochemical activity within these structures, giving rise to a balanced response of the cell to external mechanical loading. This signaling process that includes these mechanical factor is called mechanotransduction. 


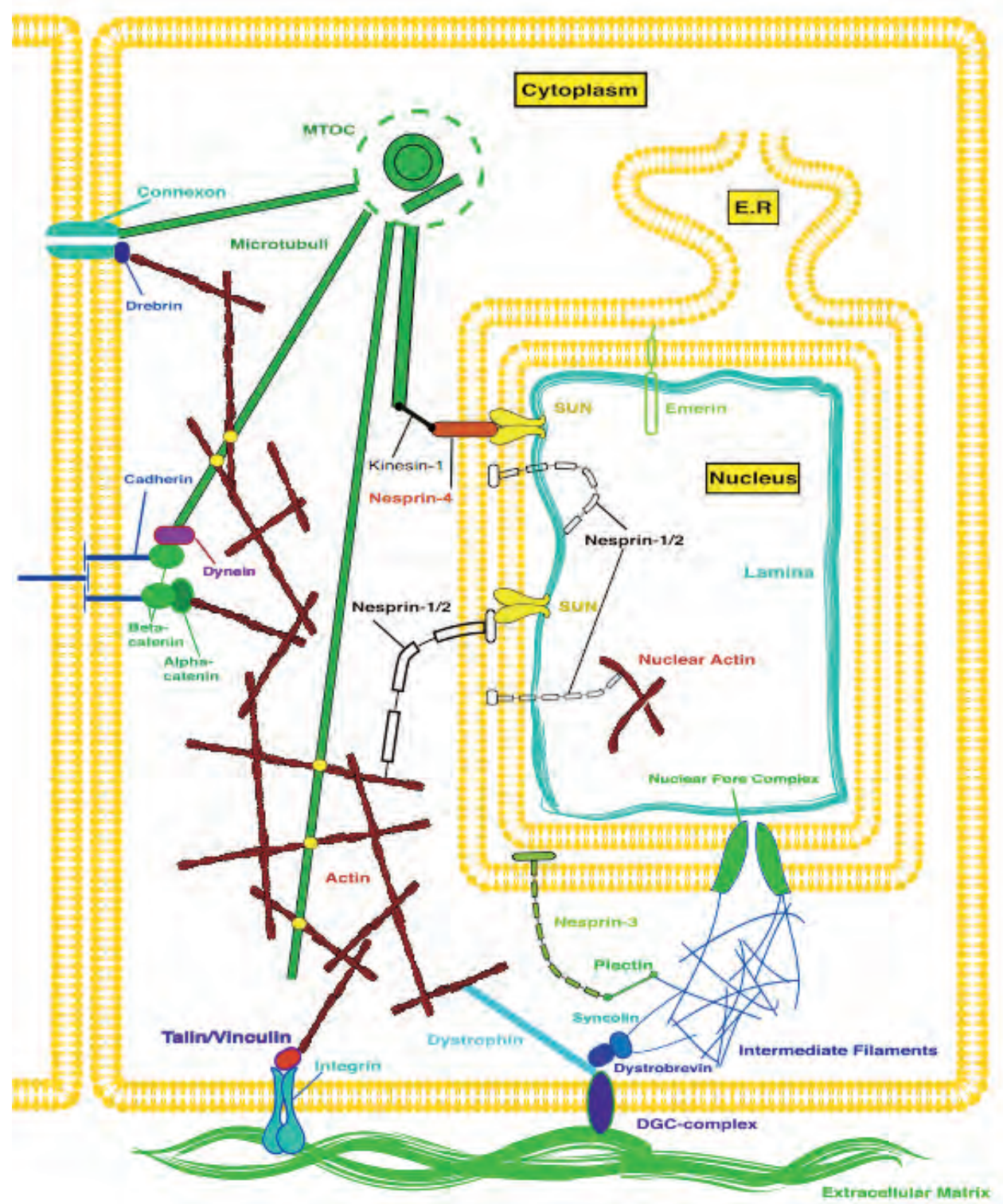

Fig. 5. Schematic representation of cellular structures involved in maintenance of the mechanical integrity of the cell. The main cytoskeletal components (microfilaments, microtubules and intermediate filaments) are key elements in linking the cellular surface to the nucleus. Cell adhesion molecules (such as cadherins and integrins) bind to the cytoskeleton through linker proteins, in this way forming connections to adherent cells or to the extracellular matrix. On the other side, the cytoskeleton is linked to the nuclear membrane (and the underlying nuclear lamina) through nesprins and SUN-proteins. All the elements form one mechanical network that functions as a prestressed scaffold that can withstand mechanical stress. 


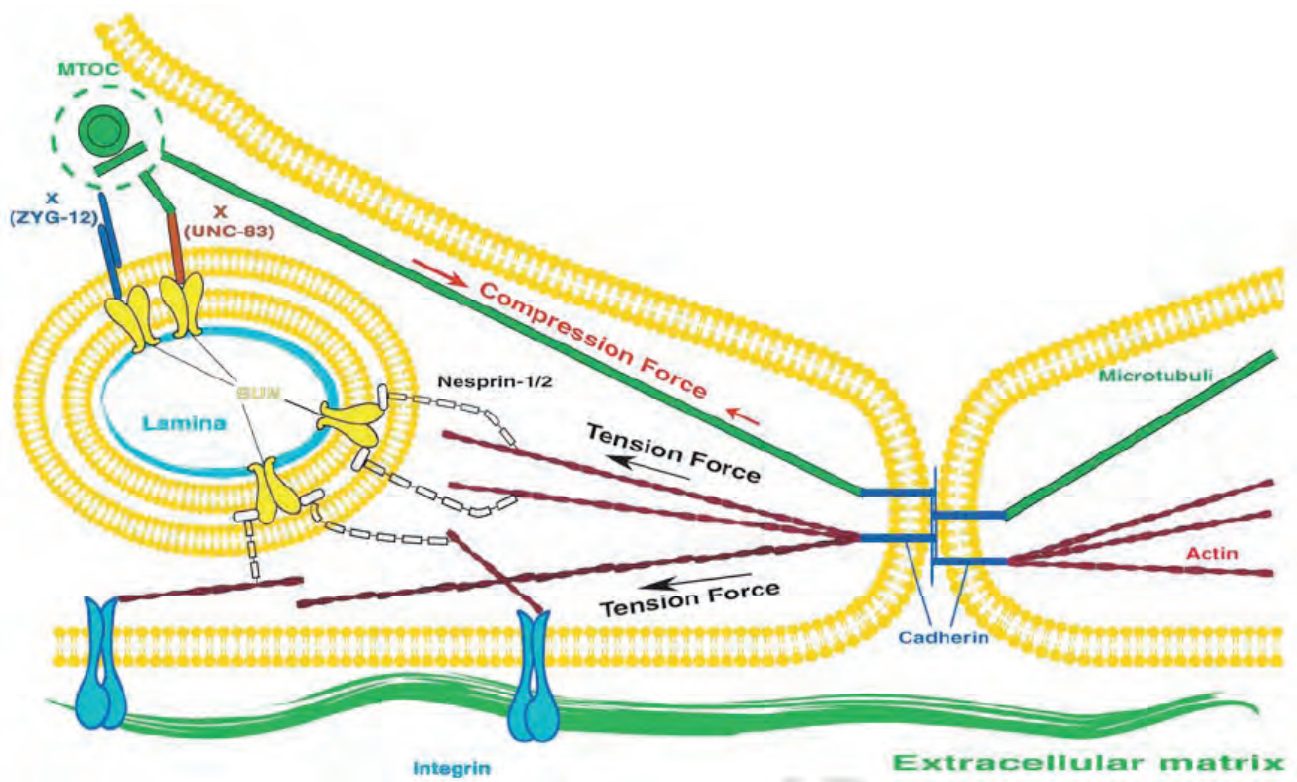

Fig. 6. Schematic representation of mechanical response in a mechanically loaded cell. The pre-stress situation of the cytoskeletal fibers differs between actin filaments which are stretched due to internal tension forces (black arrow), and microtubules which are compressed due to internal compression forces (red arrow). As these structures are linked to the cadherins and integrins, they will withstand the external mechanical stress that is exerted onto the cell. Also the nucleus plays an role in the maintenance of the cell during mechanical loading, through their connection to the cytoskeleton. These connections are formed by nesprins and SUN-proteins. As a whole, all the different mechanical elements (adhesion molecules, cytoskeleton and the nucleus) form a balanced tensegrity structure that can resist and respond to the external mechanical forces. For clarity, not all linker proteins, as shown in Fig. 5, are visualized.

\section{Mechanotransduction and signaling proteins}

When a cell receives an external stress signal, it acts by a mechanical response via the adhesion molecules and the cytoskeleton, triggering a signaling cascade throughout the cell, ultimately leading to the transcription of stress induced genes. For example, functional hemi-channels of connexins can respond to mechanical stress (mechanosensitivity), leading to intracellular calcium waves and the release of ATP from the cells into the extracellular environment [144-146].

The most important signaling pathway that is activated in response to mechanical stress is undoubtedly that of the Rho GTPases. When cells are mechanically loaded, Rho GTPases are activated by extracellular agonists or by interaction with activated adhesion molecules [147, 148]. Rho-GTPases comprise Cdc42, Rho and Rac, with extracellular agonists of these proteins being bradykinin (acts on Cdc42), Platelet- 
Derived Growth Factor (acts on Rac) and lysophosphatic acid (acts on Rho) [147]. Rho-GTPases can also activate each other. For instance, Cdc42 activates Rac, which subsequently affects Rho. Upon activation, Rho-GTPases direct the cells' response to mechanical loading by cytoskeletal rearrangement and migration of the cell. To achieve this they activate a whole series of secondary messengers such as phosphatidylinositol 3- kinase and P21-activated kinases like PAK1, Par6 and protein kinase C [149-152].

Signal transduction through the above-mentioned molecules evokes pronounced structural changes, especially in actin filament organization [147, 153-155]. Rho activates the formation of actin stress fibers and adhesion complexes by clustering of integrins and associated proteins. Rac and $\mathrm{Cdc} 42$ promote actin polymerization in the formation of lamellipodia and membrane ruffles, or filopodia and microspikes, respectively. Cdc42 promote depolymerization of actin stress fibers at the site of cell protrusions. The generation of these cellular extensions plays a role in determination of cell polarity and motility processes directing the cell away from external stress factors. Next to the effect on the actin cytoskeleton, Rho-GTPases also affect microtubular dynamics [156]. Microtubules are directed towards the direction of cell migration and add to the creation of cell polarity. Also the localization of centrosomes, from which the microtubules extend, is controlled by Rho-GTPases [157-159], which is independent of microtubular stability [159], and vimentin intermediate filaments can be reorganized by Rho GTPases [152]. Coordinated by the microtubules and mediated by $\mathrm{Cdc} 42$, the nucleus will rotate and translocate during mechanical stress [158].

Next to the effect on the cytoskeleton, the localization of the nucleus and of the centrosome, Rho GTPases affect the transcription of stress-activated genes [58, 160] [161-163]. Following activation by mechanical stimulation, mechanosensitive genes like egr-1 and iex-1 regulate cell growth and apoptosis. Also, the NFkB regulated stress response pathway is attenuated [29].

Recent studies show that the MAPK-pathway is upregulated in laminopathies due to lmna or emd mutations, as well as in lmna -/- cells [164-168].

Mice with a Lmna H222P mutation (mouse model of autosomal dominant EmeryDreifuss muscular dystrophy) show an increase in gene expression of several genes important in cell signalling pathways, including the MAPK pathways. In the hearts of these mice and in isolated cardiomyocytes an increase of the phosphorylated JNK and Erk1/2, and a translocation of these activated proteins to the nucleus, could be seen. Also upregulation of downstream targets bcl-2, Elk1 and c-Jun could be observed. The activation of these pathways in these mice leads to fibrosis and ventricular dilatation [165]. To a lesser extent, similar results could be obtained in Emd (emerin) knockout mice [165]. 
In another model for autosomal dominant Emery-Dreifuss muscular dystrophy, mouse myoblasts with Lmna R453W mutation were treated with an Erk-inhibitor. This leads to a decrease in abnormal cell death and cell proliferation and more differentiation into myocytes [164].

A direct link between the absence of A-type lamins and emerin and the enhanced phosphorylation of Erk was shown by knock-down using RNAi of A-type lamins and emerin in HeLa and $\mathrm{C} 2 \mathrm{C} 12$ cells. Also target genes of phophorylated Erk were activated. This process could be diminished by the use of a Erk-inhibitor [167]. When the mice with Lmna H222P mutation were treated with an inhibitor of Erk activation, Erk phosphorylation and activation of downstream genes in the heart were blocked, indicating that this part of the pathway was still functional and a potential target for treatment. The ventricular dilatation that $\mathrm{Lmna}^{\mathrm{H} 222 \mathrm{P} / \mathrm{H} 222 \mathrm{P}}$ mice normally developed was delayed after treatment with the Erk-inhibitor. Also, the cardiac ejection fraction returned to normal after treatment [167]., indicating a role for Erk-inhibitors in the treatment of laminopathies.

\section{Consequences of lamin deficiency}

In lamin-A deficient cells, disturbances can be found in several structural components of the cell. First, in the nucleus of such cells, abnormally shaped nuclear envelopes showing herniations or honeycomb structures of the lamina and the lamin-associated proteins can be found $[169,170]$. This is associated with mechanically weakened nuclei that rupture more easily under mechanical loading [29, 30, 58]. But these effects on the nuclei do not completely explain the massive effects observed in affected tissues, in which the coordinated cellular response is lost. This implies that a link must exist between the deregulation of the nuclear lamina, and the effects on the whole cell and affected tissue.

Three models have been proposed to explain the link between the mutation of the LMNA gene and the observed pathologies:

(A) The 'gene regulation' hypothesis states that particularly A-type lamins play a regulatory role in specific DNA transcription by activating mechanosensitive genes.

(B) The 'structural' hypothesis states that lamins play an essential role in the structural integrity of the nucleus and thus in the structural integrity of the whole cell, via connections between nuclear lamina, cytoskeleton and extracellular matrix $[29,30,171]$. This model implies also that the nuclear lamina plays a crucial role in the prestressed tensegrity model of mechanical 
balance in the cell. Next to the purely mechanical link between these structures, there is also a biochemical (signaling) link [29, 30] and as a result lamina changes can result in disturbed signaling cascades.

(C) The third hypothesis stipulates that the disturbance of the lamina results in impaired DNA repair, giving rise to premature aging of cells and tissues.

The three hypotheses do not exclude each other. Different mutations in the lamin gene might affect either the genetic pathway, the mechanical pathway, or both, in different ways.

\section{Conclusions}

The tensegrity model, in which a pre-stressed construction of nucleoskeletal proteins functions as a scaffold to resist external mechanical stresses, is based on the correct function of both the cytoskeleton and the nucleoskeleton. The nuclear lamina plays a pivotal role in both maintaining of nuclear structure and in the correct organization of cytoskeletal and adhesion structures in the cell.

\section{Acknowledgement}

We gratefully acknowledge Cathy Willem for helping with the figures. 


\section{References}

[1] D.E. Ingber, Tensegrity I. Cell structure and hierarchical systems biology, J Cell Sci 116 (2003) 1157-1173.

[2] D.E. Ingber, Tensegrity II. How structural networks influence cellular information processing networks, J Cell Sci 116 (2003) 1397-1408.

[3] C. Galli, S. Guizzardi, G. Passeri, G.M. Macaluso and R. Scandroglio, Life on the wire: on tensegrity and force balance in cells, Acta Biomed 76 (2005) 512.

[4] N. Wang, J.P. Butler and D.E. Ingber, Mechanotransduction across the cell surface and through the cytoskeleton, Science 260 (1993) 1124-1127.

[5] N. Wang, K. Naruse, D. Stamenovic, J.J. Fredberg, S.M. Mijailovich, I.M. Tolic-Norrelykke, T. Polte, R. Mannix and D.E. Ingber, Mechanical behavior in living cells consistent with the tensegrity model, Proc Natl Acad Sci U S A 98 (2001) 7765-7770.

[6] N. Wang and Z. Suo, Long-distance propagation of forces in a cell, Biochem Biophys Res Commun 328 (2005) 1133-1138.

[7] J. Mizushima-Sugano, T. Maeda and T. Miki-Noumura, Flexural rigidity of singlet microtubules estimated from statistical analysis of their contour lengths and end-to-end distances, Biochim Biophys Acta 755 (1983) 257-262.

[8] J. Pourati, A. Maniotis, D. Spiegel, J.L. Schaffer, J.P. Butler, J.J. Fredberg, D.E. Ingber, D. Stamenovic and N. Wang, Is cytoskeletal tension a major determinant of cell deformability in adherent endothelial cells?, Am J Physiol 274 (1998) C1283-1289.

[9] S. Hu, J. Chen, J.P. Butler and N. Wang, Prestress mediates force propagation into the nucleus, Biochem Biophys Res Commun 329 (2005) 423-428.

[10] C.S. Chen, J. Tan and J. Tien, Mechanotransduction at cell-matrix and cell-cell contacts, Annu Rev Biomed Eng 6 (2004) 275-302.

[11] L.H. Snoeckx, R.N. Cornelussen, F.A. Van Nieuwenhoven, R.S. Reneman and G.J. Van Der Vusse, Heat shock proteins and cardiovascular pathophysiology, Physiol Rev 81 (2001) 1461-1497.

[12] J.L. Broers, F.C. Ramaekers, G. Bonne, R.B. Yaou and C.J. Hutchison, Nuclear lamins: laminopathies and their role in premature ageing, Physiol Rev 86 (2006) 967-1008.

[13] U. Aebi, J. Cohn, L. Buhle and L. Gerace, The nuclear lamina is a meshwork of intermediate-type filaments, Nature 323 (1986) 560-564.

[14] G. Biamonti, M. Giacca, G. Perini, G. Contreas, L. Zentilin, F. Weighardt, M. Guerra, G. Della Valle, S. Saccone, S. Riva and et al., The gene for a novel human lamin maps at a highly transcribed locus of chromosome 19 which replicates at the onset of S-phase, Mol Cell Biol 12 (1992) 3499-3506. 
[15] K. Furukawa, H. Inagaki and Y. Hotta, Identification and cloning of an mRNA coding for a germ cell-specific A-type lamin in mice, Exp Cell Res 212 (1994) 426-430.

[16] J. Harborth, S.M. Elbashir, K. Bechert, T. Tuschl and K. Weber, Identification of essential genes in cultured mammalian cells using small interfering RNAs, $\mathrm{J}$ Cell Sci 114 (2001) 4557-4565.

[17] B.M. Machiels, A.H. Zorenc, J.M. Endert, H.J. Kuijpers, G.J. van Eys, F.C. Ramaekers and J.L. Broers, An alternative splicing product of the lamin A/C gene lacks exon 10, J Biol Chem 271 (1996) 9249-9253.

[18] F. Lin and H.J. Worman, Structural organization of the human gene encoding nuclear lamin A and nuclear lamin C, J Biol Chem 268 (1993) 16321-16326.

[19] F. Lin and H.J. Worman, Structural organization of the human gene (LMNB1) encoding nuclear lamin B1, Genomics 27 (1995) 230-236.

[20] J. Liu, T. Rolef Ben-Shahar, D. Riemer, M. Treinin, P. Spann, K. Weber, A. Fire and Y. Gruenbaum, Essential roles for Caenorhabditis elegans lamin gene in nuclear organization, cell cycle progression, and spatial organization of nuclear pore complexes, Mol Biol Cell 11 (2000) 3937-3947.

[21] R. Benavente, G. Krohne and W.W. Franke, Cell type-specific expression of nuclear lamina proteins during development of Xenopus laevis, Cell 41 (1985) 177-190.

[22] J.L. Broers, B.M. Machiels, H.J. Kuijpers, F. Smedts, R. van den Kieboom, Y. Raymond and F.C. Ramaekers, A- and B-type lamins are differentially expressed in normal human tissues, Histochem Cell Biol 107 (1997) 505-517.

[23] C.F. Lehner, R. Stick, H.M. Eppenberger and E.A. Nigg, Differential expression of nuclear lamin proteins during chicken development, J Cell Biol 105 (1987) 577-587.

[24] R.A. Rober, K. Weber and M. Osborn, Differential timing of nuclear lamin $\mathrm{A} / \mathrm{C}$ expression in the various organs of the mouse embryo and the young animal: a developmental study, Development 105 (1989) 365-378.

[25] R. Stick and P. Hausen, Changes in the nuclear lamina composition during early development of Xenopus laevis, Cell 41 (1985) 191-200.

[26] L. Vergnes, M. Peterfy, M.O. Bergo, S.G. Young and K. Reue, Lamin B1 is required for mouse development and nuclear integrity, Proc Natl Acad Sci U S A 101 (2004) 10428-10433.

[27] J.L. Broers, C.J. Hutchison and F.C. Ramaekers, Laminopathies, J Pathol 204 (2004) 478-488.

[28] C.J. Hutchison, M. Alvarez-Reyes and O.A. Vaughan, Lamins in disease: why do ubiquitously expressed nuclear envelope proteins give rise to tissue-specific disease phenotypes?, J Cell Sci 114 (2001) 9-19.

[29] J. Lammerding, P.C. Schulze, T. Takahashi, S. Kozlov, T. Sullivan, R.D. Kamm, C.L. Stewart and R.T. Lee, Lamin A/C deficiency causes defective nuclear mechanics and mechanotransduction, J Clin Invest 113 (2004) 370378. 
[30] J.L. Broers, E.A. Peeters, H.J. Kuijpers, J. Endert, C.V. Bouten, C.W. Oomens, F.P. Baaijens and F.C. Ramaekers, Decreased mechanical stiffness in LMNA-/- cells is caused by defective nucleo-cytoskeletal integrity: implications for the development of laminopathies, Hum Mol Genet 13 (2004) 2567-2580.

[31] K.N. Dahl, S.M. Kahn, K.L. Wilson and D.E. Discher, The nuclear envelope lamina network has elasticity and a compressibility limit suggestive of a molecular shock absorber, J Cell Sci 117 (2004) 4779-4786.

[32] N. Caille, O. Thoumine, Y. Tardy and J.J. Meister, Contribution of the nucleus to the mechanical properties of endothelial cells, J Biomech 35 (2002) 177187.

[33] F. Guilak, J.R. Tedrow and R. Burgkart, Viscoelastic properties of the cell nucleus, Biochem Biophys Res Commun 269 (2000) 781-786.

[34] K.N. Dahl, A.J. Engler, J.D. Pajerowski and D.E. Discher, Power-law rheology of isolated nuclei with deformation mapping of nuclear substructures, Biophys J 89 (2005) 2855-2864.

[35] J.M. Holaska, A.K. Kowalski and K.L. Wilson, Emerin caps the pointed end of actin filaments: evidence for an actin cortical network at the nuclear inner membrane, PLoS Biol 2 (2004) E231.

[36] G. Lattanzi, V. Cenni, S. Marmiroli, C. Capanni, E. Mattioli, L. Merlini, S. Squarzoni and N.M. Maraldi, Association of emerin with nuclear and cytoplasmic actin is regulated in differentiating myoblasts, Biochem Biophys Res Commun 303 (2003) 764-770.

[37] A.M. Sasseville and Y. Langelier, In vitro interaction of the carboxy-terminal domain of lamin A with actin, FEBS Lett 425 (1998) 485-489.

[38] S. Vlcek, T. Dechat and R. Foisner, Nuclear envelope and nuclear matrix: interactions and dynamics, Cell Mol Life Sci 58 (2001) 1758-1765.

[39] P. Hozak, A.M. Sasseville, Y. Raymond and P.R. Cook, Lamin proteins form an internal nucleoskeleton as well as a peripheral lamina in human cells, J Cell Sci 108 (1995) 635-644.

[40] G. Jagatheesan, S. Thanumalayan, B. Muralikrishna, N. Rangaraj, A.A. Karande and V.K. Parnaik, Colocalization of intranuclear lamin foci with RNA splicing factors, J Cell Sci 112 (1999) 4651-4661.

[41] J.L. Broers, B.M. Machiels, G.J. van Eys, H.J. Kuijpers, E.M. Manders, R. van Driel and F.C. Ramaekers, Dynamics of the nuclear lamina as monitored by GFP-tagged A-type lamins, J Cell Sci 112 (1999) 3463-3475.

[42] M. Fricker, M. Hollinshead, N. White and D. Vaux, Interphase nuclei of many mammalian cell types contain deep, dynamic, tubular membrane-bound invaginations of the nuclear envelope, J Cell Biol 136 (1997) 531-544.

[43] D. McClintock, L.B. Gordon and K. Djabali, Hutchinson-Gilford progeria mutant lamin A primarily targets human vascular cells as detected by an antiLamin A G608G antibody, Proc Natl Acad Sci U S A 103 (2006) 2154-2159. 
[44] N. Johnson, M. Krebs, R. Boudreau, G. Giorgi, M. LeGros and C. Larabell, Actin-filled nuclear invaginations indicate degree of cell de-differentiation, Differentiation 71 (2003) 414-424.

[45] K. Prufert, A. Vogel and G. Krohne, The lamin CxxM motif promotes nuclear membrane growth, J Cell Sci 117 (2004) 6105-6116.

[46] J.G. Conboy, Structure, function, and molecular genetics of erythroid membrane skeletal protein 4.1 in normal and abnormal red blood cells, Semin Hematol 30 (1993) 58-73.

[47] S.W. Krauss, C. Chen, S. Penman and R. Heald, Nuclear actin and protein 4.1: essential interactions during nuclear assembly in vitro, Proc Natl Acad Sci U S A 100 (2003) 10752-10757.

[48] S.W. Krauss, R. Heald, G. Lee, W. Nunomura, J.A. Gimm, N. Mohandas and J.A. Chasis, Two distinct domains of protein 4.1 critical for assembly of functional nuclei in vitro, J Biol Chem 277 (2002) 44339-44346.

[49] S.W. Krauss, C.A. Larabell, S. Lockett, P. Gascard, S. Penman, N. Mohandas and J.A. Chasis, Structural protein 4.1 in the nucleus of human cells: dynamic rearrangements during cell division, J Cell Biol 137 (1997) 275-289.

[50] C.A. Harris, P.J. Andryuk, S.W. Cline, S. Mathew, J.J. Siekierka and G. Goldstein, Structure and mapping of the human thymopoietin (TMPO) gene and relationship of human TMPO beta to rat lamin-associated polypeptide 2, Genomics 28 (1995) 198-205.

[51] T.M. Gant, C.A. Harris and K.L. Wilson, Roles of LAP2 proteins in nuclear assembly and DNA replication: truncated LAP2beta proteins alter lamina assembly, envelope formation, nuclear size, and DNA replication efficiency in Xenopus laevis extracts, J Cell Biol 144 (1999) 1083-1096.

[52] L. Yang, T. Guan and L. Gerace, Lamin-binding fragment of LAP2 inhibits increase in nuclear volume during the cell cycle and progression into $\mathrm{S}$ phase, J Cell Biol 139 (1997) 1077-1087.

[53] T. Dechat, B. Korbei, O.A. Vaughan, S. Vlcek, C.J. Hutchison and R. Foisner, Lamina-associated polypeptide 2alpha binds intranuclear A-type lamins, J Cell Sci 113 (2000) 3473-3484.

[54] T. Dechat, S. Vlcek and R. Foisner, Review: lamina-associated polypeptide 2 isoforms and related proteins in cell cycle-dependent nuclear structure dynamics, J Struct Biol 129 (2000) 335-345.

[55] D.J. Ellis, H. Jenkins, W.G. Whitfield and C.J. Hutchison, GST-lamin fusion proteins act as dominant negative mutants in Xenopus egg extract and reveal the function of the lamina in DNA replication, J Cell Sci 110 (1997) 25072518 .

[56] S. Bione, E. Maestrini, S. Rivella, M. Mancini, S. Regis, G. Romeo and D. Toniolo, Identification of a novel X-linked gene responsible for EmeryDreifuss muscular dystrophy, Nat Genet 8 (1994) 323-327.

[57] A. Vaughan, M. Alvarez-Reyes, J.M. Bridger, J.L. Broers, F.C. Ramaekers, M. Wehnert, G.E. Morris, W.G.F. Whitfield and C.J. Hutchison, Both emerin 
and lamin C depend on lamin A for localization at the nuclear envelope, J Cell Sci 114 (2001) 2577-2590.

[58] J. Lammerding, J. Hsiao, P.C. Schulze, S. Kozlov, C.L. Stewart and R.T. Lee, Abnormal nuclear shape and impaired mechanotransduction in emerindeficient cells, J Cell Biol 170 (2005) 781-791.

[59] K.L. Wilson, J.M. Holaska, R. Montes de Oca, K. Tifft, M. Zastrow, M. Segura-Totten, M. Mansharamani and L. Bengtsson, Nuclear membrane protein emerin: roles in gene regulation, actin dynamics and human disease, Novartis Found Symp 264 (2005) 51-58; discussion 58-62, 227-230.

[60] Y. Tsuchiya, A. Hase, M. Ogawa, H. Yorifuji and K. Arahata, Distinct regions specify the nuclear membrane targeting of emerin, the responsible protein for Emery-Dreifuss muscular dystrophy, Eur J Biochem 259 (1999) 859-865.

[61] J. Liu, K.K. Lee, M. Segura-Totten, E. Neufeld, K.L. Wilson and Y. Gruenbaum, MAN1 and emerin have overlapping function(s) essential for chromosome segregation and cell division in Caenorhabditis elegans, Proc Natl Acad Sci U S A 100 (2003) 4598-4603.

[62] F. Lin, D.L. Blake, I. Callebaut, I.S. Skerjanc, L. Holmer, M.W. McBurney, M. Paulin-Levasseur and H.J. Worman, MAN1, an inner nuclear membrane protein that shares the LEM domain with lamina-associated polypeptide 2 and emerin, J Biol Chem 275 (2000) 4840-4847.

[63] H.R. Waterham, J. Koster, P. Mooyer, G. Noort Gv, R.I. Kelley, W.R. Wilcox, R.J. Wanders, R.C. Hennekam and J.C. Oosterwijk, Autosomal recessive HEM/Greenberg skeletal dysplasia is caused by 3 beta-hydroxysterol delta 14reductase deficiency due to mutations in the lamin B receptor gene, Am J Hum Genet 72 (2003) 1013-1017.

[64] D. Makatsori, N. Kourmouli, H. Polioudaki, L.D. Shultz, K. McLean, P.A. Theodoropoulos, P.B. Singh and S.D. Georgatos, The inner nuclear membrane protein lamin B receptor forms distinct microdomains and links epigenetically marked chromatin to the nuclear envelope, J Biol Chem 279 (2004) 2556725573.

[65] R. Foisner, Inner nuclear membrane proteins and the nuclear lamina, J Cell Sci 114 (2001) 3791-3792.

[66] E.C. Schirmer, T. Guan and L. Gerace, Involvement of the lamin rod domain in heterotypic lamin interactions important for nuclear organization, J Cell Biol 153 (2001) 479-489.

[67] J.M. Mislow, J.M. Holaska, M.S. Kim, K.K. Lee, M. Segura-Totten, K.L. Wilson and E.M. McNally, Nesprin-1alpha self-associates and binds directly to emerin and lamin A in vitro, FEBS Lett 525 (2002) 135-140.

[68] Q. Zhang, J.N. Skepper, F. Yang, J.D. Davies, L. Hegyi, R.G. Roberts, P.L. Weissberg, J.A. Ellis and C.M. Shanahan, Nesprins: a novel family of spectrin-repeat-containing proteins that localize to the nuclear membrane in multiple tissues, J Cell Sci 114 (2001) 4485-4498. 
[69] V.C. Padmakumar, S. Abraham, S. Braune, A.A. Noegel, B. Tunggal, I. Karakesisoglou and E. Korenbaum, Enaptin, a giant actin-binding protein, is an element of the nuclear membrane and the actin cytoskeleton, Exp Cell Res 295 (2004) 330-339.

[70] Y.Y. Zhen, T. Libotte, M. Munck, A.A. Noegel and E. Korenbaum, NUANCE, a giant protein connecting the nucleus and actin cytoskeleton, $\mathrm{J}$ Cell Sci 115 (2002) 3207-3222.

[71] E.D. Apel, R.M. Lewis, R.M. Grady and J.R. Sanes, Syne-1, a dystrophin- and Klarsicht-related protein associated with synaptic nuclei at the neuromuscular junction, J Biol Chem 275 (2000) 31986-31995.

[72] K. Wilhelmsen, S.H. Litjens, I. Kuikman, N. Tshimbalanga, H. Janssen, I. van den Bout, K. Raymond and A. Sonnenberg, Nesprin-3, a novel outer nuclear membrane protein, associates with the cytoskeletal linker protein plectin, $\mathrm{J}$ Cell Biol 171 (2005) 799-810.

[73] K.J. Roux, M.L. Crisp, Q. Liu, D. Kim, S. Kozlov, C.L. Stewart and B. Burke, Nesprin 4 is an outer nuclear membrane protein that can induce kinesinmediated cell polarization, Proc Natl Acad Sci U S A 106 (2009) 2194-2199.

[74] D.A. Starr and J.A. Fischer, KASH 'n Karry: the KASH domain family of cargo-specific cytoskeletal adaptor proteins, Bioessays 27 (2005) 1136-1146.

[75] D.A. Starr and M. Han, Role of ANC-1 in tethering nuclei to the actin cytoskeleton, Science 298 (2002) 406-409.

[76] D.T. Warren, Q. Zhang, P.L. Weissberg and C.M. Shanahan, Nesprins: intracellular scaffolds that maintain cell architecture and coordinate cell function?, Expert Rev Mol Med 7 (2005) 1-15.

[77] R.M. Grady, D.A. Starr, G.L. Ackerman, J.R. Sanes and M. Han, Syne proteins anchor muscle nuclei at the neuromuscular junction, Proc Natl Acad Sci U S A 102 (2005) 4359-4364.

[78] J.M. Mislow, M.S. Kim, D.B. Davis and E.M. McNally, Myne-1, a spectrin repeat transmembrane protein of the myocyte inner nuclear membrane, interacts with lamin A/C, J Cell Sci 115 (2002) 61-70.

[79] V.C. Padmakumar, T. Libotte, W. Lu, H. Zaim, S. Abraham, A.A. Noegel, J. Gotzmann, R. Foisner and I. Karakesisoglou, The inner nuclear membrane protein Sun1 mediates the anchorage of Nesprin-2 to the nuclear envelope, J Cell Sci 118 (2005) 3419-3430.

[80] D.M. Hodzic, D.B. Yeater, L. Bengtsson, H. Otto and P.D. Stahl, Sun2 is a novel mammalian inner nuclear membrane protein, J Biol Chem 279 (2004) 25805-25812.

[81] C.J. Malone, W.D. Fixsen, H.R. Horvitz and M. Han, UNC-84 localizes to the nuclear envelope and is required for nuclear migration and anchoring during C. elegans development, Development 126 (1999) 3171-3181.

[82] I. Hagan and M. Yanagida, The product of the spindle formation gene sad1+ associates with the fission yeast spindle pole body and is essential for viability, J Cell Biol 129 (1995) 1033-1047. 
[83] D.A. Starr and M. Han, ANChors away: an actin based mechanism of nuclear positioning, J Cell Sci 116 (2003) 211-216.

[84] M. Crisp, Q. Liu, K. Roux, J.B. Rattner, C. Shanahan, B. Burke, P.D. Stahl and D. Hodzic, Coupling of the nucleus and cytoplasm: role of the LINC complex, J Cell Biol 172 (2006) 41-53.

[85] C. Buch, R. Lindberg, R. Figueroa, S. Gudise, E. Onischenko and E. Hallberg, An integral protein of the inner nuclear membrane localizes to the mitotic spindle in mammalian cells, J Cell Sci 122 (2009) 2100-2107.

[86] S. Gudise, R. Figueroa, R. Lindberg, V. Larsson and E. Hallberg, Samp1 is functionally associated with the LINCcomplex and A-type lamina networks, $\mathrm{J}$ Cell Sci 124 (2011) 2077-2085.

[87] E.C. Schirmer, L. Florens, T. Guan, J.R. Yates, 3rd and L. Gerace, Nuclear membrane proteins with potential disease links found by subtractive proteomics, Science 301 (2003) 1380-1382.

[88] E.A. Peeters, C.V. Bouten, C.W. Oomens, D.L. Bader, L.H. Snoeckx and F.P. Baaijens, Anisotropic, three-dimensional deformation of single attached cells under compression, Ann Biomed Eng 32 (2004) 1443-1452.

[89] P.A. Janmey, U. Euteneuer, P. Traub and M. Schliwa, Viscoelastic properties of vimentin compared with other filamentous biopolymer networks, J Cell Biol 113 (1991) 155-160.

[90] A.M. Malek and S. Izumo, Mechanism of endothelial cell shape change and cytoskeletal remodeling in response to fluid shear stress, J Cell Sci 109 (1996) 713-726.

[91] S. McCue, S. Noria and B.L. Langille, Shear-induced reorganization of endothelial cell cytoskeleton and adhesion complexes, Trends Cardiovasc Med 14 (2004) 143-151.

[92] T. Mizutani, H. Haga and K. Kawabata, Cellular stiffness response to external deformation: tensional homeostasis in a single fibroblast, Cell Motil Cytoskeleton 59 (2004) 242-248.

[93] M.L. Gardel, J.H. Shin, F.C. MacKintosh, L. Mahadevan, P. Matsudaira and D.A. Weitz, Elastic behavior of cross-linked and bundled actin networks, Science 304 (2004) 1301-1305.

[94] A.J. Baines and J.C. Pinder, The spectrin-associated cytoskeleton in mammalian heart, Front Biosci 10 (2005) 3020-3033.

[95] Y. Tseng, T.P. Kole, J.S. Lee, E. Fedorov, S.C. Almo, B.W. Schafer and D. Wirtz, How actin crosslinking and bundling proteins cooperate to generate an enhanced cell mechanical response, Biochem Biophys Res Commun 334 (2005) 183-192.

[96] T.A. Hornberger, D.D. Armstrong, T.J. Koh, T.J. Burkholder and K.A. Esser, Intracellular signaling specificity in response to uniaxial vs. multiaxial stretch: implications for mechanotransduction, Am J Physiol Cell Physiol 288 (2005) C185-194. 
[97] M.K. Miller, H. Granzier, E. Ehler and C.C. Gregorio, The sensitive giant: the role of titin-based stretch sensing complexes in the heart, Trends Cell Biol 14 (2004) 119-126.

[98] T.A. Schroer, Motors, clutches and brakes for membrane traffic: a commemorative review in honor of Thomas Kreis, Traffic 1 (2000) 3-10.

[99] F. Aniento, N. Emans, G. Griffiths and J. Gruenberg, Cytoplasmic dyneindependent vesicular transport from early to late endosomes, J Cell Biol 123 (1993) 1373-1387.

[100] J. Gruenberg, G. Griffiths and K.E. Howell, Characterization of the early endosome and putative endocytic carrier vesicles in vivo and with an assay of vesicle fusion in vitro, J Cell Biol 108 (1989) 1301-1316.

[101] J. Lippincott-Schwartz, Cytoskeletal proteins and Golgi dynamics, Curr Opin Cell Biol 10 (1998) 52-59.

[102] G.J. Strous, E.G. Berger, P. van Kerkhof, H. Bosshart, B. Berger and H.J. Geuze, Brefeldin A induces a microtubule-dependent fusion of galactosyltransferase-containing vesicles with the rough endoplasmic reticulum, Biol Cell 71 (1991) 25-31.

[103] N. Hirokawa, Y. Noda and Y. Okada, Kinesin and dynein superfamily proteins in organelle transport and cell division, Curr Opin Cell Biol 10 (1998) 60-73.

[104] J.H. Walenta, A.J. Didier, X. Liu and H. Kramer, The Golgi-associated hook3 protein is a member of a novel family of microtubule-binding proteins, J Cell Biol 152 (2001) 923-934.

[105] D.A. Compton, Spindle assembly in animal cells, Annu Rev Biochem 69 (2000) 95-114.

[106] V. Hunyadi, D. Chretien and I.M. Janosi, Mechanical stress induced mechanism of microtubule catastrophes, J Mol Biol 348 (2005) 927-938.

[107] J.A. Tuszynski, T. Luchko, S. Portet and J.M. Dixon, Anisotropic elastic properties of microtubules, Eur Phys J E Soft Matter 17 (2005) 29-35.

[108] D. Fatkin and R.M. Graham, Molecular mechanisms of inherited cardiomyopathies, Physiol Rev 82 (2002) 945-980.

[109] D. Stamenovic, Microtubules may harden or soften cells, depending of the extent of cell distension, J Biomech 38 (2005) 1728-1732.

[110] D. Stamenovic, S.M. Mijailovich, I.M. Tolic-Norrelykke, J. Chen and N. Wang, Cell prestress. II. Contribution of microtubules, Am J Physiol Cell Physiol 282 (2002) C617-624.

[111] L. Chang and R.D. Goldman, Intermediate filaments mediate cytoskeletal crosstalk, Nat Rev Mol Cell Biol 5 (2004) 601-613.

[112] J.E. Eriksson, T. He, A.V. Trejo-Skalli, A.S. Harmala-Brasken, J. Hellman, Y.H. Chou and R.D. Goldman, Specific in vivo phosphorylation sites determine the assembly dynamics of vimentin intermediate filaments, J Cell Sci 117 (2004) 919-932. 
[113] B.T. Helfand, L. Chang and R.D. Goldman, Intermediate filaments are dynamic and motile elements of cellular architecture, J Cell Sci 117 (2004) 133-141.

[114] T.M. Svitkina, A.B. Verkhovsky and G.G. Borisy, Plectin sidearms mediate interaction of intermediate filaments with microtubules and other components of the cytoskeleton, J Cell Biol 135 (1996) 991-1007.

[115] G. Wiche, Role of plectin in cytoskeleton organization and dynamics, J Cell Sci 111 (1998) 2477-2486.

[116] L. Borradori and A. Sonnenberg, Structure and function of hemidesmosomes: more than simple adhesion complexes, J Invest Dermatol 112 (1999) 411-418.

[117] P.A. Coulombe, M.E. Hutton, R. Vassar and E. Fuchs, A function for keratins and a common thread among different types of epidermolysis bullosa simplex diseases, J Cell Biol 115 (1991) 1661-1674.

[118] E.B. Lane and W.H. McLean, Keratins and skin disorders, J Pathol 204 (2004) 355-366.

[119] D. Paulin, A. Huet, L. Khanamyrian and Z. Xue, Desminopathies in muscle disease, J Pathol 204 (2004) 418-427.

[120] M. Pekny and M. Pekna, Astrocyte intermediate filaments in CNS pathologies and regeneration, J Pathol 204 (2004) 428-437.

[121] E. Butkevich, S. Hulsmann, D. Wenzel, T. Shirao, R. Duden and I. Majoul, Drebrin is a novel connexin-43 binding partner that links gap junctions to the submembrane cytoskeleton, Curr Biol 14 (2004) 650-658.

[122] B.N. Giepmans, I. Verlaan and W.H. Moolenaar, Connexin-43 interactions with ZO-1 and alpha- and beta-tubulin, Cell Commun Adhes 8 (2001) 219223.

[123] T. Thomas, K. Jordan and D.W. Laird, Role of cytoskeletal elements in the recruitment of $\mathrm{Cx} 43$-GFP and Cx26-YFP into gap junctions, Cell Commun Adhes 8 (2001) 231-236.

[124] K. Vleminckx and R. Kemler, Cadherins and tissue formation: integrating adhesion and signaling, Bioessays 21 (1999) 211-220.

[125] K.S. Ko, P.D. Arora and C.A. McCulloch, Cadherins mediate intercellular mechanical signaling in fibroblasts by activation of stretch-sensitive calciumpermeable channels, J Biol Chem 276 (2001) 35967-35977.

[126] M.J. Moeller, A. Soofi, G.S. Braun, X. Li, C. Watzl, W. Kriz and L.B. Holzman, Protocadherin FAT1 binds Ena/VASP proteins and is necessary for actin dynamics and cell polarization, Embo J 23 (2004) 3769-3779.

[127] E. Tzima, M. Irani-Tehrani, W.B. Kiosses, E. Dejana, D.A. Schultz, B. Engelhardt, G. Cao, H. DeLisser and M.A. Schwartz, A mechanosensory complex that mediates the endothelial cell response to fluid shear stress, Nature 437 (2005) 426-431.

[128] A. Katsumi, A.W. Orr, E. Tzima and M.A. Schwartz, Integrins in mechanotransduction, J Biol Chem 279 (2004) 12001-12004. 
[129] K. Yamada, K.G. Green, A.M. Samarel and J.E. Saffitz, Distinct pathways regulate expression of cardiac electrical and mechanical junction proteins in response to stretch, Circ Res 97 (2005) 346-353.

[130] X. Yutao, W. Geru, B. Xiaojun, G. Tao and M. Aiqun, Mechanical stretchinduced hypertrophy of neonatal rat ventricular myocytes is mediated by beta(1)-integrin-microtubule signaling pathways, Eur J Heart Fail 8 (2006) 1622.

[131] R.O. Hynes, Integrins: versatility, modulation, and signaling in cell adhesion, Cell 69 (1992) 11-25.

[132] S.H. Huang, K.J. Yang, J.C. Wu, K.J. Chang and S.M. Wang, Effects of hyperthermia on the cytoskeleton and focal adhesion proteins in a human thyroid carcinoma cell line, J Cell Biochem 75 (1999) 327-337.

[133] M.A. Schwartz and R.K. Assoian, Integrins and cell proliferation: regulation of cyclin-dependent kinases via cytoplasmic signaling pathways, J Cell Sci 114 (2001) 2553-2560.

[134] C. Chaponnier and G. Gabbiani, Pathological situations characterized by altered actin isoform expression, J Pathol 204 (2004) 386-395.

[135] E. Clarkson, C.F. Costa and L.M. Machesky, Congenital myopathies: diseases of the actin cytoskeleton, J Pathol 204 (2004) 407-417.

[136] N.J. Cairns, V.M. Lee and J.Q. Trojanowski, The cytoskeleton in neurodegenerative diseases, J Pathol 204 (2004) 438-449.

[137] R. Schroder, J. Reimann, P. Salmikangas, C.S. Clemen, Y.K. Hayashi, I. Nonaka, K. Arahata and O. Carpen, Beyond LGMD1A: myotilin is a component of central core lesions and nemaline rods, Neuromuscul Disord 13 (2003) 451-455.

[138] L. Pulkkinen, V.E. Kimonis, Y. Xu, E.N. Spanou, W.H. McLean and J. Uitto, Homozygous alpha6 integrin mutation in junctional epidermolysis bullosa with congenital duodenal atresia, Hum Mol Genet 6 (1997) 669-674.

[139] K.N. North and A.H. Beggs, Deficiency of a skeletal muscle isoform of alphaactinin (alpha-actinin-3) in merosin-positive congenital muscular dystrophy, Neuromuscul Disord 6 (1996) 229-235.

[140] W.H. McLean, L. Pulkkinen, F.J. Smith, E.L. Rugg, E.B. Lane, F. Bullrich, R.E. Burgeson, S. Amano, D.L. Hudson, K. Owaribe, J.A. McGrath, J.R. McMillan, R.A. Eady, I.M. Leigh, A.M. Christiano and J. Uitto, Loss of plectin causes epidermolysis bullosa with muscular dystrophy: cDNA cloning and genomic organization, Genes Dev 10 (1996) 1724-1735.

[141] J. Feng, J. Yan, C.H. Buzin, J.A. Towbin and S.S. Sommer, Mutations in the dystrophin gene are associated with sporadic dilated cardiomyopathy, Mol Genet Metab 77 (2002) 119-126.

[142] J.A. Rafael, G.A. Cox, K. Corrado, D. Jung, K.P. Campbell and J.S. Chamberlain, Forced expression of dystrophin deletion constructs reveals structure-function correlations, J Cell Biol 134 (1996) 93-102. 
[143] A. Bornemann and H.H. Goebel, Congenital myopathies, Brain Pathol 11 (2001) 206-217.

[144] L. Bao, F. Sachs and G. Dahl, Connexins are mechanosensitive, Am J Physiol Cell Physiol 287 (2004) C1389-1395.

[145] L. Leybaert, K. Braet, W. Vandamme, L. Cabooter, P.E. Martin and W.H. Evans, Connexin channels, connexin mimetic peptides and ATP release, Cell Commun Adhes 10 (2003) 251-257.

[146] C.E. Stout, J.L. Costantin, C.C. Naus and A.C. Charles, Intercellular calcium signaling in astrocytes via ATP release through connexin hemichannels, J Biol Chem 277 (2002) 10482-10488.

[147] D.J. Mackay and A. Hall, Rho GTPases, J Biol Chem 273 (1998) 2068520688.

[148] A.S. Torsoni, T.M. Marin, L.A. Velloso and K.G. Franchini, RhoA/ROCK signaling is critical to FAK activation by cyclic stretch in cardiac myocytes, Am J Physiol Heart Circ Physiol 289 (2005) H1488-1496.

[149] M. Fukata, M. Nakagawa and K. Kaibuchi, Roles of Rho-family GTPases in cell polarisation and directional migration, Curr Opin Cell Biol 15 (2003) 590597.

[150] M.A. Sells, A. Pfaff and J. Chernoff, Temporal and spatial distribution of activated Pak1 in fibroblasts, J Cell Biol 151 (2000) 1449-1458.

[151] J. Cau and A. Hall, Cdc42 controls the polarity of the actin and microtubule cytoskeletons through two distinct signal transduction pathways, J Cell Sci 118 (2005) 2579-2587.

[152] W. Chan, R. Kozma, Y. Yasui, M. Inagaki, T. Leung, E. Manser and L. Lim, Vimentin intermediate filament reorganization by Cdc42: involvement of PAK and p70 S6 kinase, Eur J Cell Biol 81 (2002) 692-701.

[153] T.P. Kole, Y. Tseng, I. Jiang, J.L. Katz and D. Wirtz, Intracellular mechanics of migrating fibroblasts, Mol Biol Cell 16 (2005) 328-338.

[154] R. Kozma, S. Ahmed, A. Best and L. Lim, The Ras-related protein Cdc42Hs and bradykinin promote formation of peripheral actin microspikes and filopodia in Swiss 3T3 fibroblasts, Mol Cell Biol 15 (1995) 1942-1952.

[155] C.D. Nobes and A. Hall, Rho GTPases control polarity, protrusion, and adhesion during cell movement, J Cell Biol 144 (1999) 1235-1244.

[156] T. Wittmann and C.M. Waterman-Storer, Cell motility: can Rho GTPases and microtubules point the way?, J Cell Sci 114 (2001) 3795-3803.

[157] E.R. Gomes, S. Jani and G.G. Gundersen, Nuclear movement regulated by Cdc42, MRCK, myosin, and actin flow establishes MTOC polarization in migrating cells, Cell 121 (2005) 451-463.

[158] J.S. Lee, M.I. Chang, Y. Tseng and D. Wirtz, Cdc42 mediates nucleus movement and MTOC polarization in Swiss 3 T3 fibroblasts under mechanical shear stress, Mol Biol Cell 16 (2005) 871-880.

[159] A.F. Palazzo, H.L. Joseph, Y.J. Chen, D.L. Dujardin, A.S. Alberts, K.K. Pfister, R.B. Vallee and G.G. Gundersen, Cdc42, dynein, and dynactin 
regulate MTOC reorientation independent of Rho-regulated microtubule stabilization, Curr Biol 11 (2001) 1536-1541.

[160] O.A. Coso, M. Chiariello, J.C. Yu, H. Teramoto, P. Crespo, N. Xu, T. Miki and J.S. Gutkind, The small GTP-binding proteins Rac1 and Cdc42 regulate the activity of the JNK/SAPK signaling pathway, Cell 81 (1995) 1137-1146.

[161] C.S. Hill, J. Wynne and R. Treisman, The Rho family GTPases RhoA, Rac1, and CDC42Hs regulate transcriptional activation by SRF, Cell 81 (1995) 1159-1170.

[162] A. Minden, A. Lin, F.X. Claret, A. Abo and M. Karin, Selective activation of the JNK signaling cascade and c-Jun transcriptional activity by the small GTPases Rac and Cdc42Hs, Cell 81 (1995) 1147-1157.

[163] D.J. Sulciner, K. Irani, Z.X. Yu, V.J. Ferrans, P. Goldschmidt-Clermont and T. Finkel, rac1 regulates a cytokine-stimulated, redox-dependent pathway necessary for NF-kappaB activation, Mol Cell Biol 16 (1996) 7115-7121.

[164] C. Favreau, E. Delbarre, J.C. Courvalin and B. Buendia, Differentiation of $\mathrm{C} 2 \mathrm{C} 12$ myoblasts expressing lamin A mutated at a site responsible for EmeryDreifuss muscular dystrophy is improved by inhibition of the MEK-ERK pathway and stimulation of the PI3-kinase pathway, Exp Cell Res 314 (2008) 1392-1405.

[165] A. Muchir, P. Pavlidis, G. Bonne, Y.K. Hayashi and H.J. Worman, Activation of MAPK in hearts of EMD null mice: similarities between mouse models of $\mathrm{X}$-linked and autosomal dominant Emery Dreifuss muscular dystrophy, Hum Mol Genet 16 (2007) 1884-1895.

[166] A. Muchir, P. Pavlidis, V. Decostre, A.J. Herron, T. Arimura, G. Bonne and H.J. Worman, Activation of MAPK pathways links LMNA mutations to cardiomyopathy in Emery-Dreifuss muscular dystrophy, J Clin Invest 117 (2007) 1282-1293.

[167] A. Muchir, J. Shan, G. Bonne, S.E. Lehnart and H.J. Worman, Inhibition of extracellular signal-regulated kinase signaling to prevent cardiomyopathy caused by mutation in the gene encoding A-type lamins, Hum Mol Genet 18 (2009) 241-247.

[168] A. Muchir, W. Wu and H.J. Worman, Reduced expression of A-type lamins and emerin activates extracellular signal-regulated kinase in cultured cells, Biochim Biophys Acta 1792 (2009) 75-81.

[169] A. Muchir, J. Medioni, M. Laluc, C. Massart, T. Arimura, A.J. van der Kooi, I. Desguerre, M. Mayer, X. Ferrer, S. Briault, M. Hirano, H.J. Worman, A. Mallet, M. Wehnert, K. Schwartz and G. Bonne, Nuclear envelope alterations in fibroblasts from patients with muscular dystrophy, cardiomyopathy, and partial lipodystrophy carrying lamin A/C gene mutations, Muscle Nerve 30 (2004) 444-450.

[170] A. Muchir, B.G. van Engelen, M. Lammens, J.M. Mislow, E. McNally, K. Schwartz and G. Bonne, Nuclear envelope alterations in fibroblasts from 
LGMD1B patients carrying nonsense Y259X heterozygous or homozygous mutation in lamin A/C gene, Exp Cell Res 291 (2003) 352-362.

[171] J.L. Broers, H.J. Kuijpers, C. Ostlund, H.J. Worman, J. Endert and F.C. Ramaekers, Both lamin A and lamin C mutations cause lamina instability as well as loss of internal nuclear lamin organization, Exp Cell Res 304 (2005) 582-592. 


\section{Chapter 2}

\section{Disturbed nuclear orientation and cellular migration in A-type lamin deficient cells.}

F. Houben, C.H.M.P.Willems, I.L.J.Declercq, K. Hochstenbach, M.A. Kamps, L.H.E.H. Snoeckx, F.C.S. Ramaekers, J.L.V. Broers

Published in part in:

Biochimica Biophysica Acta - Molecular Cell Research; 1793 (2009) 312-324 


\section{Abstract}

The nuclear lamina and the cytoskeleton form an integrated structure that warrants proper mechanical functioning of cells. We have studied the correlation between structural alterations and migrational behaviour in fibroblasts with and without A-type lamins. We show that loss of A-type lamins causes loss of emerin and nesprin-3 from the nuclear envelope, concurring with a disturbance in the connection between the nucleus and the cytoskeleton in A-type lamin deficient (Imna -/-) cells. In these cells functional migration assays during in vitro wound healing revealed a delayed reorientation of the nucleus and MTOC during migration, as well as a loss of nuclear oscillatory rotation. These observations in fibroblasts isolated from lmna knockout mice were confirmed in a $3 \mathrm{~T} 3$ cell line with stable reduction of lmna expression due to RNAi approach.

Our results indicate that A-type lamins play a key role in maintaining directional movement governed by the cytoskeleton, and that the loss of these karyoskeletal proteins has important consequences for functioning of the cell as a mechanical entity. 


\section{Introduction}

The nuclear lamina is a network of type- $\mathrm{V}$ intermediate filaments located at the inner nuclear membrane of eukaryotic cells, with the major protein constituents comprising A-and B-type lamins. Lamins play an important role in maintaining the structural integrity of the cell, by forming a complex network in the nucleus and by the formation of a bridge between the nucleus and the cellular membrane via the cytoskeleton [1]. Lamins have also been suggested to modulate gene expression during e.g. cell differentiation [2, 3] and mechanotransduction [4]. The lamin A/C gene (LMNA in humans and lmna in mice) encodes for at least four different splice variants i.e. lamin A, lamin A 10, lamin C and lamin C2 (for a recent review see [5]).

Several proteins, located in the outer and inner nuclear membrane, mediate the linkage of the nuclear membrane and lamina to the different cytoskeletal components. Most nesprin isoforms are associated with the outer nuclear membrane and are linked to the nuclear lamina through SUN-proteins, which are located in the perinuclear space, forming a complex called linker of nucleoskeleton and cytoskeleton (LINC) [6]. In cells lacking A-type lamins (lmna -/- cells) aberrations in the organization of the cytoskeleton can be found which indicate that the link between the cytoskeleton and the nucleus is A-type lamin-dependent [7]. The absence of A-type lamins has a severe impact on the mechanical properties of the nucleus and the cell as a whole, which becomes apparent upon mechanical compression [8], and mechanical stretch [7, 9]. While upon compression nuclei of normal fibroblasts deform anisotropically, nuclei of Imna -/- fibroblasts deform isotropically, indicating a disturbed nucleo-cytoskeletal interaction $[7,9]$. This is supported by the finding that disconnecting the wild type (wt) nucleus from the mechanical tension of the cytoskeleton scaffold results also in isotropic nuclear deformation as in the lmna -/- cells [9].

Since an intact cytoskeleton organization is critical for cell migration we now examined whether or not the disturbed nucleo-cytoskeletal interaction in lmna -/- cells interferes with the in vitro migratory behaviour of fibroblasts in wound healing experiments. To this end we compared wt fibroblasts with lamin A/C knockout and lamin A/C knockdown fibroblasts. Normally, during this process the cytoskeleton is reorganized to establish spatial polarity and to form membrane protrusions facing the direction of migration [10]. Actin rearrangements are essential for cellular migration and are shown to be mediated by Rho GTPases [11]. When cells are activated to migrate, the membrane at the leading edge shows ruffling with microfilaments containing lamellipodia and filopodia [11]. Microfilaments are linked to lamins 
through the LINC-complex, composed of nesprin-1 and -2 and SUN-proteins [6, 1217].

Next to the microfilaments also microtubules are essential in the process of wound healing [10], as they are selectively stabilized near the leading edge [18-20], allowing the formation of membrane protrusions at this side of the cell [21, 22]. During the migration process microtubules also play a role in the rotation and translocation of the nucleus and the reorientation of the microtubule organizing centre (MTOC) [23, 24]. In C. elegans microtubules and the nuclear lamina interconnect through the UNC-83 SUN family protein [25-28], while the Hook family protein ZYG-12 links the nucleus to the MTOC [29, 30]. In Drosophila the MTOC and the microtubules are linked to the nuclear lamina through the nesprin-like Klarsicht protein [31]. Although so far no vertebrate homologues of these proteins have been described, the data from C. elegans and Drosophila suggest a tight binding of the MTOC and the microtubules to the nucleus via lamins (for a review see [32]). Wilhelmsen et al. [33] hypothesized that also nesprin- 3 may link microtubules to the vertebrate nucleus.

While the structure of both microfilaments and microtubules shows abnormalities in lmna -/- cells, even more pronounced abnormalities have been described for the intermediate filament organization [7, 9, 34]. Intermediate filaments are linked to the nuclear envelope via plectin and the recently discovered nesprin-3 [35]. During cell migration intermediate filaments play an essential role in interactions through (hemi)desmosomes between adjacent epithelial cells and with the extracellular matrix [36]. Therefore, abnormalities in the structure of intermediate filaments, due to the absence of A-type lamins, could lead to a disturbance in cellular dynamics. Through their effects on cytoskeletal filament organization lamin-mutations even appear to affect the organization of cell membrane-associated proteins. For example, in transgenic mice with a lmna-N195K mutation, a mislocalization of connexin-43 was detected in cardiac tissue [37], which could explain the conduction defects found in laminopathies [5].

It is thus conceivable that changes in the nuclear lamina have consequences for structural protein functioning throughout the whole cell. In the present study we show that the absence of A-type lamins indeed affects the functional organization of the cytoskeleton, leading to defects in nuclear and MTOC reorientation, resulting in an uncoordinated response to wounding. 


\section{Materials \& methods}

\section{Cell cultures}

Wild type (wt) and A-type lamin-deficient (lmna -/-) mouse embryonic fibroblasts (MEFs) were kind gifts from B. Burke (Department of Anatomy and Cell Biology, University of Florida, Florida, USA) and C.L. Stewart (Laboratory of Cancer and Developmental Biology, National Cancer Institute, Maryland, USA) [38].

3 T3 cells (CCL-92) were obtained from the American Type Culture Collection.

Cells were cultured in Dulbecco's modified Eagle's medium (DMEM) (Gibco Life Technologies Ltd., Paisley, UK) containing 10\% fetal calf serum (FCS, HyClone Laboratories, Logan, Utah, USA), $1 \mathrm{mM}$ L-glutamine and $0.1 \%$ gentamycin (Eurovet, Bladel, the Netherlands) at $37^{\circ} \mathrm{C}$ in a humidified $5 \% \mathrm{CO} 2$ incubator. Cells were passaged by splitting at $1: 3$ to $1: 5$ ratios using a $0.125 \%$ trypsin (Invitrogen Life Technologies, Breda, The Netherlands) $/ 0.02 \mathrm{M}$ EDTA $/ 0.02 \%$ glucose solution in phosphate buffered saline (pH 7.4, PBS).

For starvation medium, the amount of fetal calf serum was reduced from $10 \%$ to $0.5 \%$.

\section{RNA interference}

Stable shRNA-based knockdown of lamin A/C was performed in 3T3 fibroblasts, using a lentiviral construct. Based on the successful knockdown of mouse lmna by Kudlow [39] we designed the following oligonucleotides:

Forward:

5'-

CACCGCTTGACTTCCAGAAGAACATTTCAAGAGAATGTTCTTCTGGAAGTC AAGC-3,

Reverse:

5 -

AAAAGCTTGACTTCCAGAAGAACATTCTCTTGAAATGTTCTTCTGGAAGTC AAGC-3'.

Using the Gateway cloning system (Invitrogen) the annealed oligonucleotides were ligated into the pENTRTM/H1/TO vector (Invitrogen). Next, the H1/TO RNAi cassette was transferred into the pLenti4/BLOCK-iT ${ }^{\mathrm{TM}}-\mathrm{DEST}$ vector (Invitrogen) using a LR recombination reaction according to the vendor's instructions. The correct incorporation of the H1/TO lmna RNAi cassette was verified by sequencing. 3T3 cells were transfected using Lipofectamin-2000 (Invitrogen), and zeocin (Invitrogen, 150 $\mu \mathrm{g} / \mathrm{ml}$ ) was used to select the transfected cells To obtain a stable reduction of A-type lamin expression cells were subcloned by limited dilution and single cell colonies with variable A-type lamin expression were selected. Two different clones, one with a 
partial knockdown (pkd) and the other with an almost complete knockdown (kd) of lamin $\mathrm{A} / \mathrm{C}$, were used for our experiments.

\section{Immunofluorescence microscopy}

MEF and 3T3 cells grown on glass coverslips were either fixed for $10 \mathrm{~min}$ with methanol $\left(-20^{\circ} \mathrm{C}\right)$, or for 15 min with $3.7 \%$ formaldehyde in PBS at room temperature followed by permeabilization for 15 min with $0.1 \%$ Triton X-100 (BDH, Poole, UK) in PBS. Primary antibodies, diluted in PBS containing $3 \%$ bovine serum albumin (BSA) (Roche Diagnostics, Mannheim, Germany), were applied during $60 \mathrm{~min}$. The following antibodies were used:

- Lamin A mouse monoclonal antibody 133A2 (IgG3, 1:100, MUbio Products B.V., Maastricht, The Netherlands).

- Lamin C rabbit polyclonal antibody RalC, (1:20, MUbio Products B.V., Maastricht, The Netherlands).

- Lamin B1 rabbit polyclonal antibody LB1 (1:200; a kind gift from J.C. Courvalin, INSERM, Paris, France).

- Lamin A, A $\Delta 10$ and C mouse monoclonal antibody X-67 (IgG1, 1:250, a kind gift from G. Krohne,Würzburg, Germany).

- Emerin mouse monoclonal antibody NCL-emerin (IgG1, dilution 1: 60; Novocastra, Newcastle upon Tyne, UK).

- $\quad \beta$-actin mouse monoclonal antibody AC-15 (IgG1, 1:1500, Sigma-Aldrich, St. Louis, Missouri, USA).

- $\quad \beta$-tubulin mouse monoclonal antibody E7 (IgG1, 1:25, Developmental Studies Hybridoma Bank, Iowa City, Iowa, USA)

- $\quad \gamma$-tubulin mouse monoclonal antibody GTU-88 (IgG1, 1:3000, Sigma-Aldrich) for MTOC staining.

- $\alpha$-tubulin polyclonal rabbit antibody (1:100, was a kind gift from M. Borgers, Janssen Pharmaceutica, Beerse, Belgium)

- EB1 monoclonal mouse antibody (EB1, IgG1, 1:100, BD Transduction Laboratories, San Jose, CA, USA).

- Vinculin monoclonal mouse antibody (hVIN-1, IgG1, 1:200, Sigma-Aldrich)

- Connexin-43 monoclonal mouse antibody (GJA1, IgM, 1:1000, Abcam, Cambridge, UK)

- Pan-cadherin monoclonal mouse antibody (CH19, IgG1, 1:500, SigmaAldrich) 
- Monoclonal mouse antibody against the dephosphorylated form of $\beta$-catenin (8E7, IgG1/k, 1:100, Upstate Cell Signalling Solutions, Lake Placid, NY, USA).

- Phospho-p44/42 MAPK (Erk1/2) rabbit monoclonal antibody 4370 (IgG, 1:2000, Cell Signal, Beverly, Massachusetts, USA).

- Vimentin mouse monoclonal antibody BV-1118 (IgM, 1:10, was a kind gift from C. Viebahn, University of Göttingen, Göttingen, Germany).

- Nesprin-3 rabbit polyclonal antibody (1:100, a kind gift from A. Sonnenberg, Netherlands Cancer Institute, Amsterdam, the Netherlands).

- SERCA2 ATPase monoclonal mouse antibody IID8 (IgG1, 1:100, Affinity BioReagents, Golden, Colorado, USA)

After washing with PBS ( $3 \times 5 \mathrm{~min}$ ), secondary antibodies were applied during $60 \mathrm{~min}$. at room temperature. Secondary antibodies used were FITC-conjugated rabbit antimouse Ig (1:50, DAKO, Glostrup, Denmark), FITC-conjugated goat anti-rabbit Ig (1:50, SBA/ITK Birmingham, AL, USA), FITC-conjugated swine anti-rabbit Ig (1:50, DAKO), Texas Red-conjugated rabbit anti-mouse Ig (SBA/ITK) and Texas Red conjugated goat anti-rabbit Ig (1:50, SBA/ITK). For double labelling, we used the appropriate combination of primary and secondary antibodies labelled with FITC and Texas Red, or a combination of immunolabelling and Texas Red conjugated Phalloidin (dilution 1:100; Molecular Probes, Leiden, The Netherlands) for the detection of actin stress fibers. After another series of washing steps, cells were mounted in $90 \%$ glycerol, containing $20 \mathrm{mM}$ Tris- $\mathrm{HCl} \mathrm{pH} 8.0,0.2 \% \mathrm{NaN} 3$, and 2\% 1,4-di-azobicyclo(2,2,2)-octane (DABCO; Merck, Darmstadt, Germany) and $0.1 \mathrm{mg} / \mathrm{ml}$ RNAse (Serva, Heidelberg, Germany). Nuclei were counterstained using diamidino-2-phenylindole $(0.5 \mu \mathrm{g} / \mathrm{ml}$ DAPI; Sigma-Aldrich) or propidium iodide $(0.5 \mu \mathrm{g} / \mathrm{ml} \mathrm{PI}$; Molecular Probes) in mounting medium. Cells were visualized using a BioRad MRC600 confocal laser scanning fluorescence microscope (BioRad Laboratories Ltd., Hemel Hempstead, UK). Some of the acquired images were deconvoluted using Huygens System image restoration software (Huygens 2.5.1a-64, Scientific Volume Imaging B.V., Hilversum, the Netherlands).

\section{Flow cytometry}

Flow cytometry was performed as described previously [40]. In brief, for each cell line equal amounts of methanol fixed cells were incubated in suspension with the lamin $\mathrm{A}$ antibody 133A2, followed by rabbit anti-mouse Ig-FITC and analyzed using a FACSort flow cytometer and Cellquest analysis software (Becton Dickinson, Sunnyvale, CA). 


\section{Western Blotting}

Samples from cell lines were lysed with RIPA-buffer $(30 \mathrm{mM}$ Tris-HCL, $50 \mathrm{mM}$ $\mathrm{NaCl}, 1 \% \mathrm{NP} 40,0.05 \%$ sodium deoxycholate, $0,1 \%$ sodium dodecyl sulfate (SDS) on ice for $30 \mathrm{~min}$. Sample buffer $(62.5 \mathrm{mM}$ Tris-HCL, 10\% glycerol, $2.3 \%$ SDS, $5 \% \beta$ mercaptoethanol, $0.05 \%$ bromophenol blue) was added to the sample $(1: 1)$.

The sample was sonicated using a Soniprep 150 sonicator (MSE Scientific Instruments, Crowley, UK) for 4 times 5 seconds. After boiling and centrifugation, the samples were used for gel electrophoresis $(1 \mathrm{hr}$ at $200 \mathrm{~V})$ in $10 \%$ SDS polyacrylamide gels using a Mini-Protean II system (Bio-Rad Laboratories Ltd, Hemel Hempstead, UK) and blotted onto nitrocellulose sheets (Protran BA85, Schleicher and Schuell Bioscience, Dassel, Germany) for $1 \mathrm{hr}$ at $100 \mathrm{~V}$ (transfer buffer: $25 \mathrm{mM}$ Tris, $190 \mathrm{mM}$ glycine, $20 \% \mathrm{v} / \mathrm{v}$ methanol, $0.02 \% \mathrm{SDS}$ ).

Immunochemical detection was performed as described [41] using the X-67 antibody against lamin $\mathrm{A}$ and $\mathrm{C}$, the $\mathrm{P} 42 / 44$ antibody against phospho-Erk1/2, peroxidaseconjugated rabbit anti-mouse Ig and swine anti-rabbit secondary antibody (DAKO, Glostrup, Denmark) and an enhanced chemiluminescence western blotting detection kit (ECL, Amersham, UK). X-67 was used instead of the 133A2 antibody against lamin A because the latter antibody does perform well on immunofluorescence but poorly in western blotting of mouse proteins.

Immunoblots were quantitatively analyzed using ImageJ software version $1.37 \mathrm{v}, \mathrm{U} . \mathrm{S}$. National Institutes of Health, Bethesda, Maryland, USA, http://rsb.info.nih.gov/ij/, 1997-2007). Immunoblot X-ray films were scanned with a Canoscan 9900F scanner (Canon) and, after calibration with a Kodak no. 3 photographic step tablet (Eastman Kodak Company, Rochester, New York, USA), integrated optical density (IOD) values for all peaks were calculated. Actin signal was used for the correction of the amount of protein loaded onto the gels.

\section{Nucleo-cytoskeletal extractions}

Wt MEFs and lmna -/- MEFs were trypsinized, resuspended in DMEM containing $10 \%$ FCS and centrifuged at $300 \mathrm{xg}$. To study the nuclear interaction of actin and vimentin, cells were extracted for 15 min with ice-cold isotonic buffer $(0.5 \%$ Triton-X$100,3 \%$ BSA in PBS). Extraction was stopped by adding 9 volumes of PBS containing 3\% BSA and a protease-inhibitor mix (Complete Mini EDTA-free, Roche Diagnostics) during 10 minutes at room temperature. After centrifugation at $300 \mathrm{xg}$ and resuspension in DMEM containing 10\% FCS, the nuclei were centrifuged onto glass slides at 500 rpm during 5 min using a Cytospin-3 centrifuge (Shandon, Zeist, the Netherlands). 
Cells were air-dried for 30 minutes, fixed for 10 min using methanol $\left(-20^{\circ} \mathrm{C}\right)$ for actin and vimentin detection, or $3.7 \%$ formaldehyde in PBS at room temperature for the lamin B staining. Alternatively, in order to prevent tubulin polymer degradation, cells were extracted at room temperature with isotonic buffer and immediately fixed for 15 min using a $3.7 \%$ formaldehyde solution in PBS. Fixed cells were centrifuged at 300xg, resuspended in DMEM containing10\% FCS and cytospinned as described above. Cells were air-dried for 1 hour, immunostained for $\beta$-tubulin. Cell preparations were visualized using confocal laser scanning fluorescence microscopy.

\section{Quantifications of immunofluorescence labelling.}

For the quantifications of the nesprin-3 staining, we used the following categories:

- Strong nesprin-3 staining in the nuclear envelope: cells with a regular staining at the nuclear envelope stronger than the staining of the cytoplasm around the nucleus (representative cell in Fig. 4C).

- Cytoplasmic staining: a stronger staining of the cytoplasm around the nucleus than the staining of the nuclear envelope (representative cell in Fig. 4D).

- Distinct loss of staining in part of the nuclear envelope (representative cell in Fig. 4E).

Actin, tubulin and vimentin association to nuclei of the extracted cells was evaluated in a semiquantitative manner and classified into the following three categories:

- A homogeneous perinuclear staining with cytoskeletal elements attached to more than $90 \%$ of nuclear rim;

- A heterogeneous perinuclear staining of cytoskeletal elements attached from 20 to $90 \%$ of nuclear surface);

- Bald nuclei, with cytoskeletal elements attached to less than $20 \%$ of nuclear surface.

For each sample, two independent observers evaluated 5 different microscopic view fields in which in each area 100 cells were randomly selected.

\section{Wound healing assay}

MEF and 3T3 cells were trypsinized and seeded onto glass coverslips. When a confluent monolayer of cells was reached the monolayer was wounded by scratching with a glass needle with a diameter of $0.6 \mathrm{~mm}$. Live cell recording during wound healing was initiated immediately after scratching. Cells were visualized using an inverted automated microscope (Leica DMRBE, Mannheim, Germany) equipped with a black and white CCD-camera (CA4742-95, Hamamatsu, Bridgewater, NJ, USA). 
Image acquisition was achieved using Openlab software (Improvision, Lexington, MA, USA). Microscopic differential interference contrast (DIC) images were generated every 30 seconds for up to 8 hours. During the vital imaging experiments, the cells were kept at $37^{\circ} \mathrm{C}$ and $5 \% \mathrm{CO} 2$.

\section{Image analysis of DIC recordings}

Independent time-lapse recordings from wound healing experiments in wt and lmna -/MEF cell cultures (5 recordings each) and in wt and lamin A/C knockdown 3T3 cell cultures (4 recordings each) were analyzed for nuclear reorientation and oscillatory rotations. 129 wt MEFs, 117 lmna -/- MEFs, 83 wt 3T3 cells, 70 intermediate and 60 strong lamin $\mathrm{A} / \mathrm{C}$ knockdown $3 \mathrm{~T} 3$ cells in the two front layers of the wound edge cells were examined and scored. The maximal nuclear rotation was calculated based on the alterations of the initial orientation of nucleoli towards the wound edge. For the calculations, we did not incorporate data from nuclei that did not rotate (rotation of 0 degrees). In addition, for selected cells the nuclear rotation was tracked using a particle tracking plug-in (MTrackJ, Erik Meijering, Biomedical Imaging Group Rotterdam, the Netherlands) and ImageJ software. A line connecting two nucleoli per cell was used to calculate the angular displacement (degrees) of the rotating nucleus in time.

\section{Reorientation of the MTOC}

The MTOC localization was determined by staining the cells for g-tubulin at $0,5,10$, 15,30 and 60 minutes (MEF) or at $0,1,2,3,4$ or 5 hours (3T3) after wounding. For determination of the MTOC orientation for each cell a line parallel to the wound edge was drawn and MTOCs were scored to have a positive reorientation when localized at the side of the nucleus facing the wound edge. At each time point in total 200 cells were analyzed by two independent observers in at least four different areas of the wounded edge.

\section{Statisticall analysis}

All calculations are expressed as mean \pm standard error, and statistical comparisons for the rotation analysis are based on Student's t-tests. 


\section{Results}

In this study we have used two different cell systems to examine the effects of the loss of A-type lamin expression on cell behaviour. First, we have produced a set of stable 3T3 sublines with a constant level of reduction of A-type lamin expression obtained by means of RNAi, followed by single cell colony selection. Secondly, we have used MEFs derived from lamin knockout mice [38], and compared these with their wt counterparts.

\section{Stable A-type lamin knockdown in 3T3 cells}

Subcloning of cells transfected with the A-type lamin RNAi vector has resulted in the generation of $3 \mathrm{~T} 3$ cell sublines with a stable reduction of A-type lamin expression as determined by immunocytochemistry. At passage 15 after subcloning the reduction of A-type lamin expression was virtually identical to the knockdown level of the original clones at passage 1 (data not shown). Here we describe our findings with one clone with a partial knockdown of lmna (pkd) and a second clone with an almost complete knockdown of A-type lamin expression $(\mathrm{kd})$.

The morphology of the 3T3 cells was altered after knockdown of A-type lamin. The wt cells are more spindle-shaped and less tightly connected to each other as compared to the knockdown cells as can be seen in DIC images of the wt, pkd and 3T3 kd cells (Fig. 1A-C). Also, kd cells grow considerably more flattened compared to wt 3T3 cells, resulting in a prominent increase in nuclear diameter. In addition, the number of abnormally elongated or otherwise deformed nuclei was considerably higher in $\mathrm{kd}$ cultures as compared to wt cells, similar to previous lamin kd studies [39].

Immunofluorescence labelling of cells demonstrated a prominent reduction of A-type lamin expression (Fig. 1D-F). Strikingly, even within these clones derived from a single cell, a considerable heterogeneity in lamin A/C expression can be still observed. In many cells the level of reduction of A-type lamin expression was, however, sufficient to induce loss of emerin from the nuclear envelope (Fig. 1G-K). While the pkd cells showed only few cells with complete nuclear emerin loss (Fig. 1H, arrow), the kd clone showed an almost complete loss of emerin from the nuclei in nearly all cells (Fig 1I). A direct correlation between the reduction of lamin A expression and the loss of emerin from the 

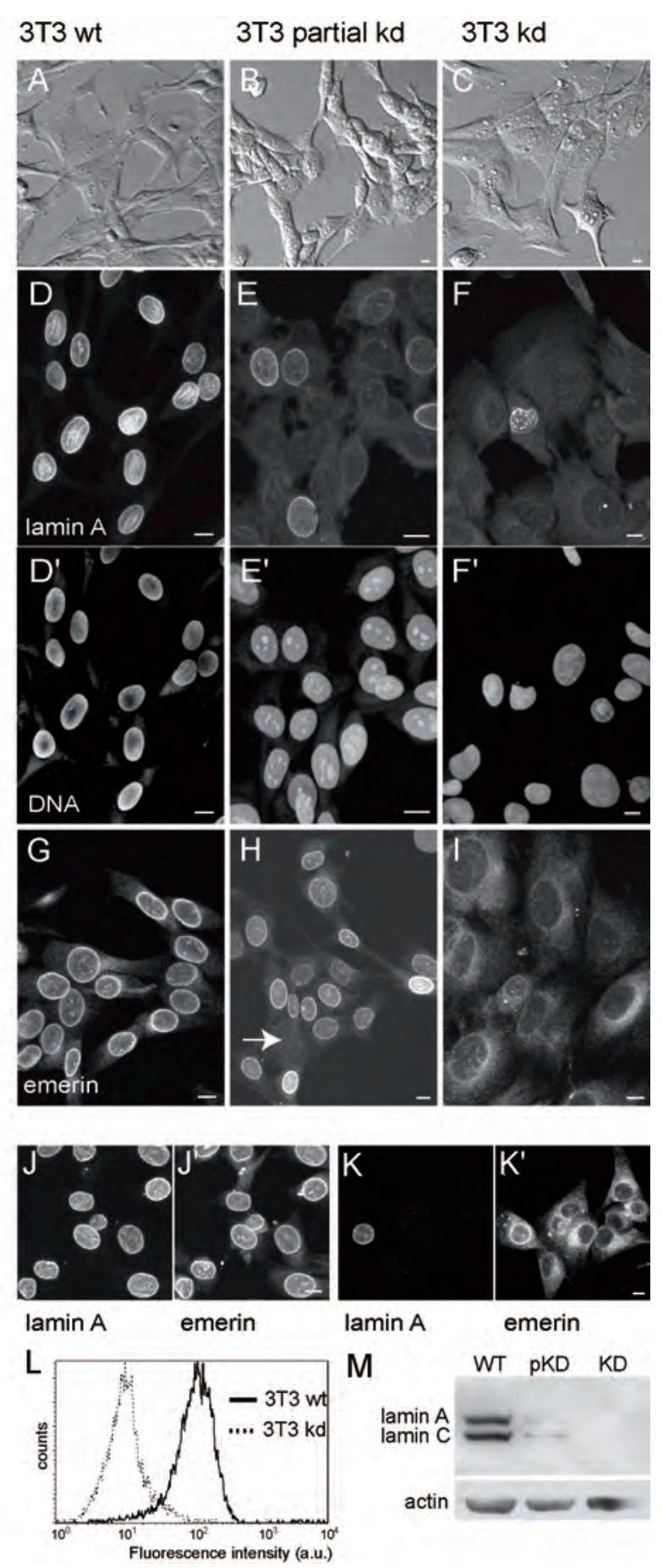
Figure 1: Effects of A-type lamin RNAi treatment on 3T3 cells. (A-C) DIC images showing differences in cell morphology between wt 3T3 cells (A) and cells with partial knockdown (pkd, B) or almost total lamin $\mathrm{A} / \mathrm{C}$ knockdown $(\mathrm{kd}, \mathrm{C})$. The latter cells are less spindle-shaped, have larger, more flattened nuclei, and are more tightly associated with each other than the wt cells. (D-K) Immunofluorescence detection using the lamin A antibody 133A2 (D-F, J,K) and the emerin antibody (G-I, J',K') in wt 3 T3 cells (D, G, J), pkd cells (E, H) and kd cells (F, I, K). Lamin A fluorescent cells are counterstained with propidium iodide (D', E', F'). Note the prominent loss of lamin A in many pkd and most kd cells. In these clones also emerin is lost in some $\left(\mathrm{H}\right.$, arrow), or most nuclei (I). Double immunofluorescence of lamin $(\mathrm{J}, \mathrm{K})$ and emerin $\left(\mathrm{J}\right.$ ', $\left.\mathrm{K}^{\prime}\right)$ shows the relocation of emerin to the endoplasmic reticulum in cells with low levels of lamin A expression in the kd clone (compare J, J' with $\mathrm{K}, \mathrm{K}^{\prime}$ ). (L) Flow cytometric detection revealed an average 6-fold and median 10-fold decrease of lamin A expression in 3T3 knockdown cells using antibody 133A2. (M) Western blot analysis using the lamin $\mathrm{A} / \mathrm{C}$ antibody $\mathrm{X}-67$, demonstrating a reduction of lamin $\mathrm{A}$ in the $3 \mathrm{~T} 3$ pkd clone to $7.8 \%$ and lamin $\mathrm{C}$ in the pkd clone to $8.2 \%$ of the original amount of wt $3 \mathrm{~T} 3$, while in the $3 \mathrm{~T} 3$ $\mathrm{kd}$ lamin $\mathrm{C}$ expression was barely visible $(0.8 \%$ of the amount found in wt $3 \mathrm{~T} 3)$ and lamin A expression was not detectable. Actin was used to verify the protein amounts loaded onto the gel. Scale bars represent $10 \mu \mathrm{m}$

nuclear envelope could be demonstrated at the single cell level by means of doublelabel immunofluorescence (Fig. 1J and 1K).

Quantitative examination of lamin A expression in individual cells by flow cytometry (Fig. 1L) revealed that the 3T3 A-type lamin kd clone has a tenfold decrease of median lamin A expression level with a large variety in expression levels between cells (113 arbitrary units (A.U.) for wt $3 \mathrm{~T} 3$ versus 10 A.U. for $3 \mathrm{~T} 3 \mathrm{kd}$, with on average a 6-fold reduction (149 \pm 366 A.U. for wt $3 \mathrm{~T} 3$ and $24 \pm 221$ A.U. for $3 \mathrm{~T} 3 \mathrm{kd}$ ).

These results could be confirmed by western blot analysis (Fig. 1M). Quantitative analysis showed a prominent reduction of both lamin $\mathrm{A}$ and $\mathrm{C}$ expression in the RNAi treated lines using the X-67 antibody. Compared to wt 3T3 cells (100\%), the relative amount of lamin expression was $7.8 \%$ for lamin A and $8.2 \%$ for lamin $\mathrm{C}$ in $3 \mathrm{~T} 3 \mathrm{pkd}$ cells, while the $3 \mathrm{~T} 3 \mathrm{kd}$ clone showed a reduction of lamin $\mathrm{C}$ expression to $0.8 \%$ of the wt value. At this exposure lamin A expression was not detectable in the kd clone. Prolonged exposure of the X-ray film did reveal a weak lamin A band (data not shown).

\section{Changes in linkage of the cytoskeleton to the nucleus}

When comparing lmna -/- to wt MEFs we confirmed that the absence of lamin A/C was associated with aberrations in the structure of the different cytoskeletal filaments (Fig. 2). In lmna -/- cells actin immunofluorescence revealed a prominent reduction of stress fibers in the perinuclear region, often with a speckled distribution of actin around the nucleus, which suggests actin depolymerisation in these areas (compare Figs. 2A 
and 2E). Geodesic dome-like organizations of stress fibers were occasionally observed surrounding the nucleus of wt cells, but not in lmna -/- cells (not shown).

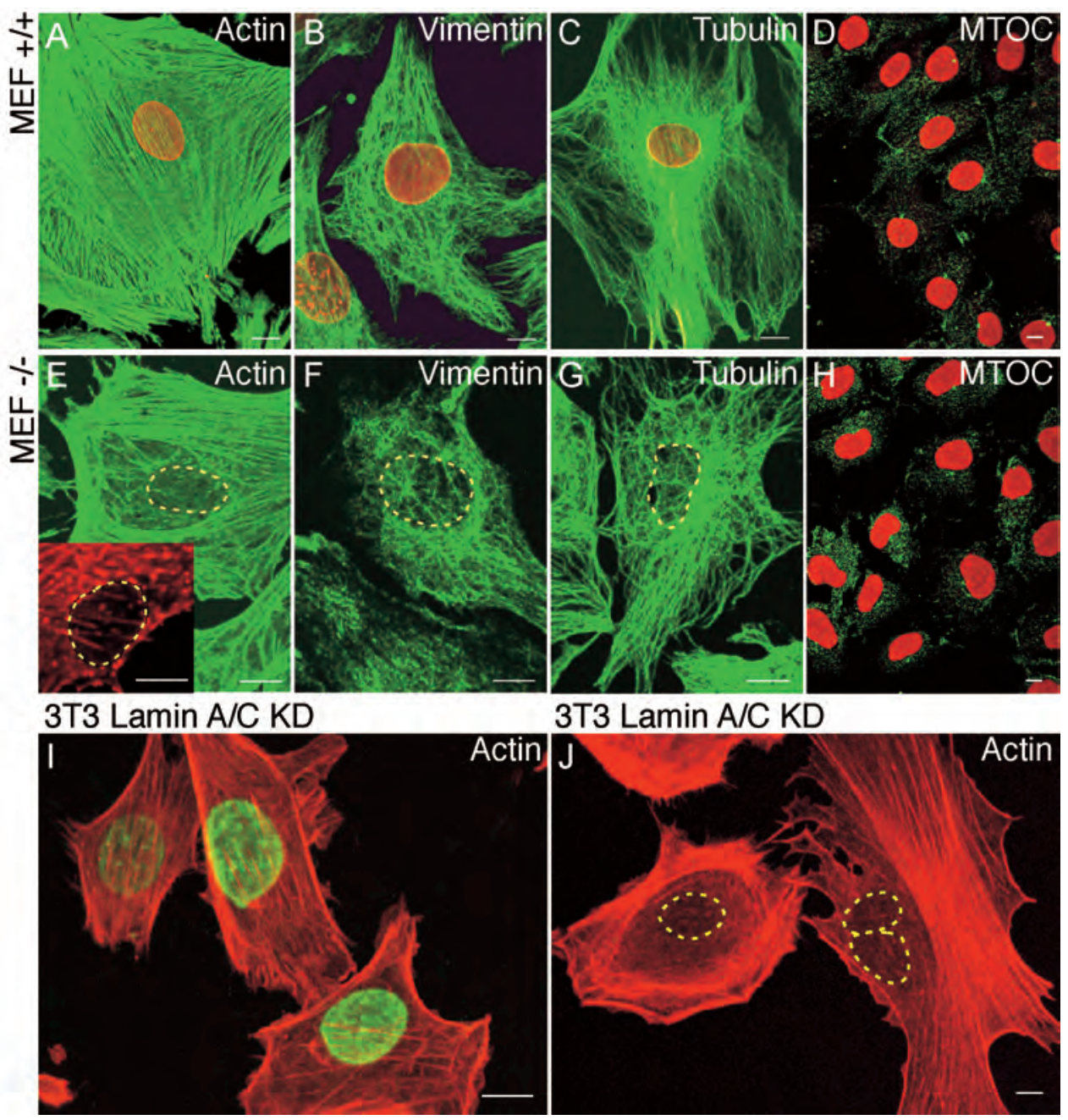

Figure 2: Loss of cytoskeleton organization due to loss of A-type lamins. Wild type (A-D) and lmna -/- (EH) MEFs immunocytochemical stained for lamin $\mathrm{C}$ in red and cytoskeletal structures in green: actin (A, E), vimentin (B, F), tubulin (C, G) or the MTOC (D, H). In lmna -/- MEF cells, a yellow dashed line outlines the nuclear border. In wt MEFs a well-structured organization of the actin cytoskeleton around the nucleus is seen (A). In the lmna -/- MEFs, the actin cytoskeleton is locally detached from the nucleus (E). F-actin labelling by Phalloidin-Texas-Red revealed a speckled distribution of actin around the lmna -/- nucleus (insert in E). Vimentin organization is highly irregular in lmna -/- MEFs (F), compared to wt MEFs (B). In wt MEFs microtubules branch from the MTOC to the cellular periphery and wrap closely around the nucleus (C), while in lmna -/- MEFs the microtubules are much more loosely arranged around the nucleus (G). In lmna -/ MEFs (H) the average distance of the MTOC to the nucleus was 1.9x larger (arrows) than 
in wt cells (D). Wt (I) and A-type lamin kd (J) 3T3 cells were double labelled for lamin A (green) and with Phalloidin-Texas Red (red). Note again the absence of F-actin fibers in areas surrounding the nucleus in lamin $3 \mathrm{~T} 3 \mathrm{kd}$ cells. Scale bars represent $10 \mu \mathrm{m}$.

Vimentin labelling revealed gross structural alterations of cytoplasmic intermediate filaments in Imna -/- cells. These were often unequally distributed throughout the cell with a strong prevalence for clustering on one side of the nucleus, and with a prominent lack of fibers in other areas of the cell (compare Fig. 2B with 2F). In addition, lmna -/- cells showed often clustering or absence of vimentin filaments in particular regions of the cytoplasm. Tubulin labelling showed less prominent differences between wt and lmna -/- cells, although we did find more lmna -/- cells with an uneven distribution of tubulin fibers throughout the cell, and decreased concentrations of microtubules in large parts of these cells (compare Figs. 2C and 2G). Compared to the lmna -/- MEF cells, the 3T3 lamin A/C knockdown cells showed a milder disturbance of distribution and organization of the different cytoskeletal elements, except for actin. While wt $3 \mathrm{~T} 3$ showed a normal actin pattern (Fig 2I), the $3 \mathrm{~T} 3 \mathrm{kd}$ cells revealed alterations of the cellular distribution of actin similar to lmna -/MEFs (compare Fig. 2J to Fig. 2E). Areas surrounding the nucleus were devoid of actin fibers, and nuclei showed a prominent eccentric localization. Distribution of the vimentin and tubulin networks did not show noticeable structural aberrations in the 3T3 A-type lamin kd cells (data not shown). The average distance of the MTOC to the nucleus was found to be almost doubled in lmna -/- MEF cells $(3.3 \pm 0.19 \mu \mathrm{m}, \mathrm{n}=120)$ as compared to wt MEFs $(1.72 \pm 0.12 \mu \mathrm{m}, \mathrm{n}=120$; Figs. $1 \mathrm{D}, \mathrm{H})$. Also in the RNAi treated $3 \mathrm{~T} 3$ cells a larger distance of the MTOC to the nucleus was observed when compared to wt cells. The average distance of the MTOC to the nucleus in 3T3 A-type lamin kd cells was $2.01 \pm 0.20 \mu \mathrm{m}$, whereas in wt $3 \mathrm{~T} 3$ cells the average distance was $0.86 \pm 0.07 \mu \mathrm{m}$. Double immunofluorescence showed that there was no correlation between the distance of the MTOC to the nucleus and localized disturbances in the nuclear lamina (data not shown).

The effects of absence of A-type lamins on the association of the cytoskeletal filaments with the nucleus were confirmed in cellular extraction studies in MEF cells. In wt MEFs actin filaments remained closely associated with most of the nuclei after isotonic extraction (Fig. 3A). In contrast, the actin filaments that remained attached to the nuclei of extracted lmna -/- MEFs (Fig. 3B) showed an uneven distribution at the nuclear periphery, and were often completely absent in large areas of the nuclear surface. 
Quantification of the staining patterns of actin after isotonic extraction (Table 1, Figure 3I) showed that $77 \pm 1 \%$ of the wt nuclei exhibit a homogeneous staining of actin around the nuclei, compared to $50 \pm 2 \%$ of the lmna -/- nuclei. $19 \pm 1 \%$ of the wt nuclei showed partial absence of actin fibers at the nuclear surface,
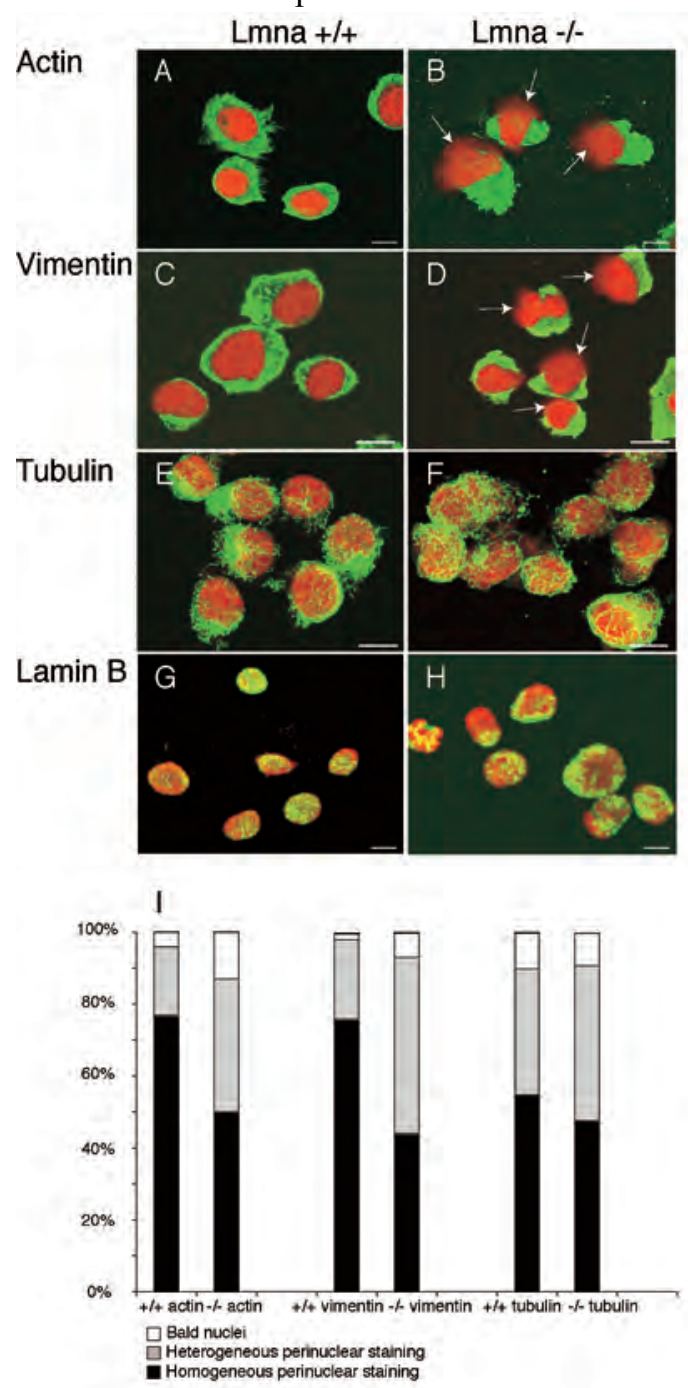

Figure 3: Analysis of cytoskeletal retainment in extracted nuclei. Isolated nuclei of wt (A, C, D, E) and lmna -/- (B, D, F, H) MEFs were stained by immunofluorescence for actin (A, B), vimentin (C, D), tubulin $(\mathrm{E}, \mathrm{F})$ and lamin $\mathrm{B}(\mathrm{G}, \mathrm{H})$ in green. Propidium iodide (red) was used to counterstain nuclei. The actin cytoskeletal filaments were less dense and showed more detachment from the isolated nuclei of lmna -/MEFs (B) compared to the nuclei of wt MEFs (A). Lmna -/- MEF nuclei showed large regions of the nucleus that were not surrounded by vimentin (D), in contrast to the distribution in wt cells (C). The 
staining pattern of tubulin in both wt (E) and lmna -/- MEFs (F) after isotonic extraction demonstrated smaller differences between the two cell types. Staining with lamin B $(G, H)$ clearly indicated that the nuclei of both cell types remained largely intact after the extraction procedure. Quantifications of actin, vimentin and tubulin association to the nucleus after detergent extraction are shown in (I). All scale bars are $10 \mu \mathrm{m}$.

compared to $37 \pm 2 \%$ of the lmna -/- nuclei. Only $4 \pm 1 \%$ of the wt nuclei showed massive absence of actin staining, or no perinuclear staining at all (bald nuclei), while $13 \pm 1 \%$ of the lmna $-/$ - nuclei showed this pattern.

Similar results were obtained for vimentin, with a uniform distribution around the nuclei of extracted wt MEFs (Fig. 3C), and absence of vimentin at large regions of the lmna -/- nuclei (Fig. 3D). $75 \pm 2 \%$ of the wt nuclei were identified with a uniform perinuclear vimentin staining, compared to $44 \pm 2 \%$ of the lmna $-/$ - nuclei. $22 \pm 1 \%$ of the wt nuclei showed partial loss of perinuclear vimentin, compared to $49 \pm 2 \%$ of the lmna -/- nuclei. Only $2 \pm 1 \%$ of the wt nuclei showed massive absence in vimentin staining, or no staining at all (bald nuclei), while $7 \pm 1 \%$ of the $l m n a-/$ - nuclei showed this pattern.

Compared to the differences found in actin and vimentin between both cell types, the differences for tubulin were less obvious (Fig. 3E, F). Quantification of the staining patterns of tubulin after isotonic extraction resulted in $55 \pm 3 \%$ of the wt nuclei with a homogeneous staining of tubulin around the nuclei, compared to $48 \pm 4 \%$ of the lmna /- nuclei. $35 \pm 3 \%$ of the wt nuclei showed partial absence of microtubules at the nuclear surface, compared to $43 \pm 2 \%$ of the lmna $-/$ - nuclei. $10 \pm 1 \%$ of the wt nuclei showed massive absence of tubulin staining, or no perinuclear staining at all (bald nuclei), while $9 \pm 2 \%$ of the lmna $-/$ - nuclei showed this pattern.

Staining with lamin B after nuclear extraction demonstrated that the nuclei of both cell types remained largely intact (Fig 3G, H).

Table 1

Quantative analysis of cytoskeletal retainment to nuclei extracted of MEFs

\begin{tabular}{|c|c|c|c|c|}
\hline & & Homogeneous staining & Heterogeneous staining & Bald nuclei \\
\hline \multirow[t]{2}{*}{ Actin } & Lmna $+/+$ & $77 \pm 1 \%$ & $19 \pm 1 \%$ & $4 \pm 1 \%$ \\
\hline & Lmna -/- & $50 \pm 2 \%$ & $37 \pm 2 \%$ & $13 \pm 1 \%$ \\
\hline \multirow[t]{2}{*}{ Vimentin } & Lmna $+/+$ & $75 \pm 2 \%$ & $22 \pm 1 \%$ & $2 \pm 1 \%$ \\
\hline & Lmna -/- & $44 \pm 2 \%$ & $49 \pm 2 \%$ & $7 \pm 1 \%$ \\
\hline \multirow[t]{2}{*}{ Tubulin } & Lmna $+/+$ & $55 \pm 3 \%$ & $35 \pm 3 \%$ & $10 \pm 1 \%$ \\
\hline & Lmna -/- & $48 \pm 4 \%$ & $43 \pm 2 \%$ & $9 \pm 2 \%$ \\
\hline
\end{tabular}




\section{Redistribution of nesprin-3 in the absence of lamin A/C}

Triggered by the disorganization of the cytoskeletal components around the nucleus, we performed a detailed study on the localization of nesprin-3, known
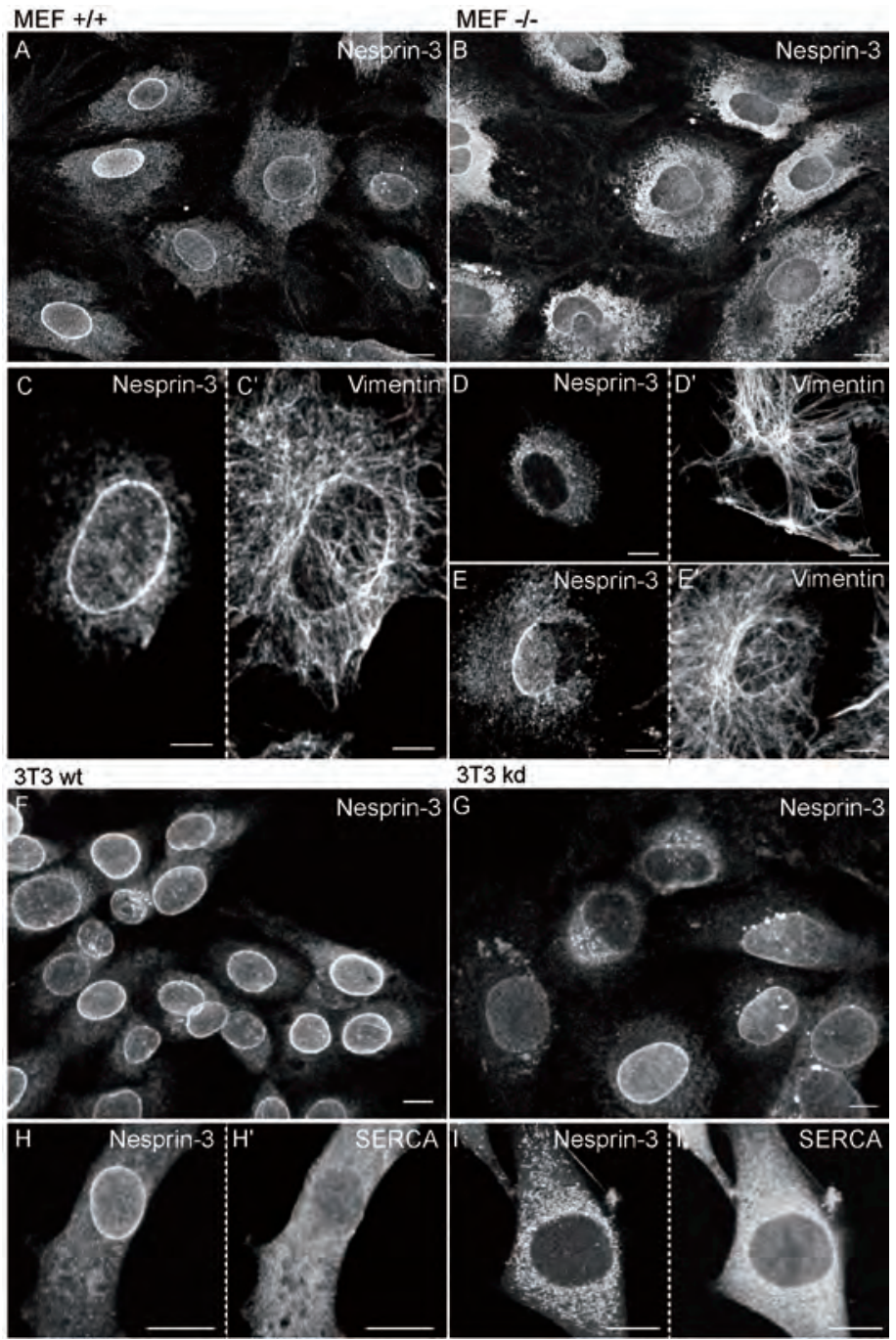

Figure 4: Disorganization of nesprin-3 in lmna -/- MEFs and 3T3 kd cells. Wt (A, C) and lmna -/- MEFs (B, D-E) were analysed for nesprin-3 (A-E) and vimentin (C'-E') localization. The immunolabelling of nesprin-3 in wt MEFs shows a brightly stained nuclear envelope $(A, C)$ while the vimentin cytoskeleton that connects to nesprin-3 is associated to the nucleus (C'). In the lmna -/- MEFs two patterns of nesprin-3 localization are observed, i.e. a cytoplasmic staining in some cells (D) and a partial labelling of the nuclear 
envelope next to a cytoplasmic staining in other cells (E). Vimentin labelling shows that the structure of vimentin around the nucleus in lmna -/- MEFs is uneven disturbed in cells with cytoplasmic nesprin-3 staining (D'). In cells with partial nesprin-3 nuclear labelling, vimentin structures are only disturbed at the location of the nesprin-3 aberrations (E'). Comparison of nesprin-3 immunofluorescence in wt 3T3 (F) and A-type lamin $\mathrm{kd}(\mathrm{G}) 3 \mathrm{~T} 3$ cells showed a partial loss of nesprin-3 from the nuclear envelope in the absence of lamin A/C. Nesprin-3 fluorescence only partially co localizes with the endoplasmic reticulum in $3 \mathrm{~T} 3 \mathrm{kd}$ cells as can be concluded from double immunofluorescence of nesprin-3 and SERCA2 (H-I). All scale bars are $10 \mu \mathrm{m}$.

to interact with intermediate filaments. While nearly all wt MEFs and $3 \mathrm{~T} 3$ cells show a prominent labelling of the nuclear rim (Figs. 4A, H), most lmna -/- MEFs and 3T3 Atype lamin kd cells showed a relocalization of nesprin-3 out of the nuclear envelope (Figs. 4B, I), which partially colocalizes with SERCA2, a marker for the endoplasmic reticulum (Figs. 4H', I'). Quantitatively, $71 \pm 3 \%$ of the wt MEFs demonstrated a strong nesprin-3 staining in the nuclear envelope, while $29 \pm 3 \%$ demonstrated more staining in the cytoplasm. In the lmna -/- cells, $47 \pm 8 \%$ of the cells showed a strong staining of nesprin-3 in the nuclear envelope, $34 \pm 8 \%$ showed only a cytoplasmic staining and $19 \pm 5 \%$ showed a distinct localization at one side of the nucleus only.

Co-staining of nesprin-3 and vimentin demonstrated that nesprin-3 was tightly associated to the nuclear envelope in most of the wt MEFs (Fig. 4C), with the vimentin structure in these cells showing a dense layer directly around the nucleus (Fig. 4C'). In the lmna -/- cells we observed remaining nesprin-3 staining in distinct regions of the nuclear envelope next to a staining of nesprin-3 in the cytoplasm (Figs. 4D, E). In the regions of these cells where the nuclear membrane was devoid of nesprin-3 also the association of vimentin intermediate filaments to the nucleus was seriously affected (Figs. 4D' and 4E').

Similar results were found for the $3 \mathrm{~T} 3 \mathrm{kd}$ cell labelling with nesprin-3. While all wt $3 \mathrm{~T} 3$ cells show a prominent labelling of the nuclear rim (Fig 4F), $75 \pm 1 \%$ of the $3 \mathrm{~T} 3$ pkd cells showed a strong staining of the nuclear envelope (not shown), $22 \pm 1 \%$ showed a more perinuclear staining and $3 \pm 1 \%$ showed localized aberrations at one side of the nucleus. Of the $3 \mathrm{~T} 3 \mathrm{kd}$ clone with almost no lamin A/C expression (Fig $4 \mathrm{G}), 61 \pm 1 \%$ of the cells showed still a strong nesprin-3 staining of the nuclear envelope, $37 \pm 1 \%$ showed a cytoplasmic staining and $2 \pm 0 \%$ showed distinct localized aberrations at one side of the nucleus. Quantification of the staining patterns

\section{Table 2}

Redistribution of nesprin-3 in lamin-deficient fibroblast

\begin{tabular}{llll}
\hline & $\begin{array}{l}\text { Nuclear envelope } \\
\text { staining }\end{array}$ & $\begin{array}{l}\text { Perinuclear } \\
\text { staining }\end{array}$ & $\begin{array}{l}\text { Localized aberrations } \\
\text { at one side of the nucleus }\end{array}$ \\
\hline MEF $+/+$ & $71 \pm 3 \%$ & $29 \pm 3 \%$ & $0 \pm 0 \%$ \\
MEF -/- & $47 \pm 8 \%$ & $34 \pm 8 \%$ & $19 \pm 5 \%$
\end{tabular}


of nesprin-3 is summarized in Table 2.

\section{Loss of nuclear re-orientation and oscillations in A-type lamin deficient cells during cellular migration in the in vitro wound healing assay}

We have investigated changes in the migratory behaviour of the wt and A-type lamin deficient cells in response to wounding of confluent monolayers by time-lapse recordings. In addition, the organization of cytoskeletal elements was studied after fixation at given time points during wound healing by immunofluorescence labelling. We found that both wt and lmna -/- MEF cells at the wound edge moved into the wounded area. On average, wound closure by lmna -/ MEFs was faster ( $3 \mathrm{hrs}$ ) than by wt MEFs ( 5 hrs). Wound healing experiments with both wt $3 \mathrm{~T} 3$ and $3 \mathrm{~T} 3 \mathrm{kd}$ cells showed that both cell lines exhibited impaired wound healing behaviour. Wound closure was much slower than the MEFs ( $>8$ hours) and incomplete in both 3T3 lines. Movement of cells appeared to be more or less random, with cells moving in and out of the wounded area. Closer examination of cell culture behaviour revealed that both lines partly lack contact inhibition, a phenomenon often seen in 3T3 cells, which are repeatedly grown to confluency. Yet, comparison of the cellular and nuclear dynamics of these cells revealed prominent differences (see below).

During the initial phase of migration towards the wound edge the nuclei of wt MEFs reoriented actively (Fig.5A). While $60 \pm 3 \%$ of the wt MEFs demonstrated this active initial nuclear repositioning within 30 minutes after wounding, only $7 \pm 1 \%$ of the lmna -/- MEFs showed the same behaviour ( $\mathrm{p}=0.00002$ ) (Fig. 5A, movies 1 and 2, Supplementary Material). The differences in this initial nuclear rotation of wt versus lmna -/- cells became very apparent when the fibroblasts suddenly repolarized towards the wound edge. In the wt cells the nucleus rotated prior to a change in the direction of migration (Fig. 5F, movie 3 of the Supplementary Material), while the nuclei of the lmna -/ cells passively followed changes in migrational direction (see movie 2, Supplementary Material).

In the second phase of the wound healing process, wt MEFs, but not the lmna -/MEFs, showed typical nuclear oscillatory rotations during cellular migration. Tracking of representative wt and lmna -/- cells illustrates the differences in oscillatory behaviour (Fig. 5B, the complete time lapse recordings are supplied as Supplementary Material, see movies 1 and 2). While $66 \pm 3 \%$ of the wt MEFs demonstrated these nuclear oscillations during cellular migration, only $9 \pm 1 \%$ of the lmna -/- MEFs showed this behaviour $(\mathrm{p}=0.000001)$ (Fig. 5B). 
While wt $3 \mathrm{~T} 3$ cells are characterized by excessive nuclear rotation, both in the beginning of migration $(67 \pm 2 \%)$ as during the migration $(59 \pm 4 \%)$, the reorientation $(20 \pm 8 \%, \mathrm{p}=0.0073)$ and oscillatory rotation $(17 \pm 1 \%, \mathrm{p}=0.00036)$ of nuclei in the $3 \mathrm{~T} 3$ lamin $\mathrm{A} / \mathrm{C}$ kd clone were strongly reduced, while in the pkd cells an intermediate nuclear reorientation $(51 \pm 4 \%, \mathrm{p}=0.022)$ and oscillatory behaviour $(40 \pm 1 \%, \mathrm{p}=$ 0.013 ) was observed (for two representative examples see Fig. 5C, full recordings can be seen in Supplementary Material, movies 4-5).

Calculations of the net rotation angle of cells in time revealed that most lmna -/- MEFs showed a gradual drift of their nuclear orientation during migration, while wt MEFs retained their nuclear orientation. (Fig. 5D, compare also movies 1 and 3 to movie 2, Supplementary Material). The same holds true for the comparison between wt and the $3 \mathrm{~T} 3 \mathrm{kd}$ cells (Fig. 5E, Supplementary Material movies 4-5).

We observed that during the wound healing process the nuclei of both wt $3 \mathrm{~T} 3$ and wt MEFs had a larger maximal rotation then the lamin A/C deficient cells. Wt $3 \mathrm{~T} 3$ cells showed a maximal rotation of $298 \pm 42$ degrees, compared to $133 \pm 17$ degrees in $3 \mathrm{~T} 3$ pkd cells and $60 \pm 10$ degrees in $3 \mathrm{~T} 3 \mathrm{kd}$ cells. Wt MEFs showed a maximal rotation of $95 \pm 5$ degrees, compared to $56 \pm 6$ degrees in lmna -/- MEFs.

\section{MTOC reorientation during migration in the in vitro wound healing assay}

The reorientation of the nucleus could be confirmed by immunofluorescent labelling of the MTOC at given time points after wounding, showing reorientation towards the wound edge (Fig. 6). Immediately after induction of the wound MTOCs were randomly located around the nuclei, i.e. $50 \%$ of the MTOCs were oriented towards the wound edge in both cell types. Examination of MTOC reorientation at different time points after wounding revealed a maximal MTOC reorientation in the wt MEFs within 30 minutes (Fig. 6A, B), while the MTOC in the lmna -/- MEFs responded slower, with a maximal reorientation after one hour (Fig. 6A, C). As predicted by the excessive nuclear rotation of $3 \mathrm{~T} 3$ cells, and the lack of directional movement of these cells, only a modest MTOC reorientation was observed, with percentages of cells oriented towards the wound edge ranging from $50-60 \%$ in all stages after wounding. The $3 \mathrm{~T} 3$ kd cell line showed similar results (data not shown). 
A
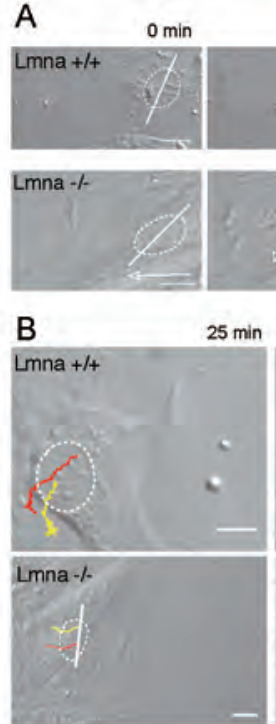

C.

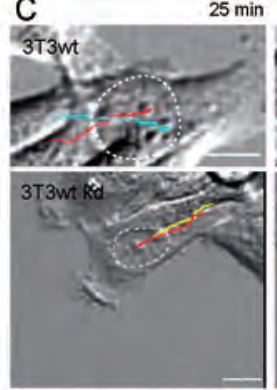

D D

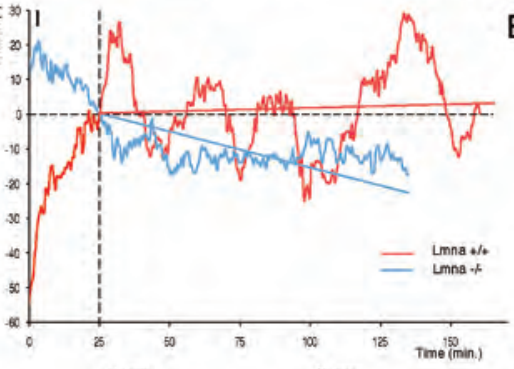

$100 \mathrm{~min}$
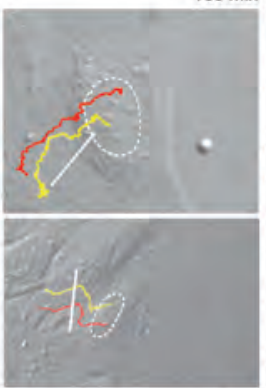

$75 \mathrm{~min}$

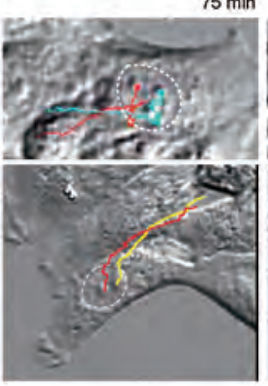

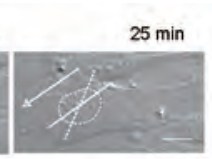
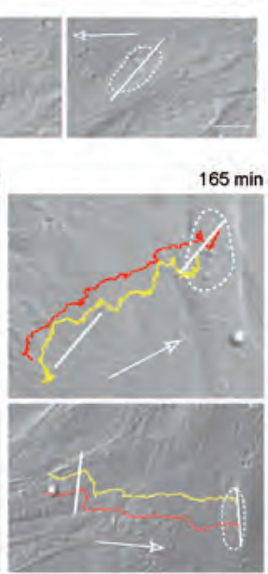

$120 \mathrm{~min}$

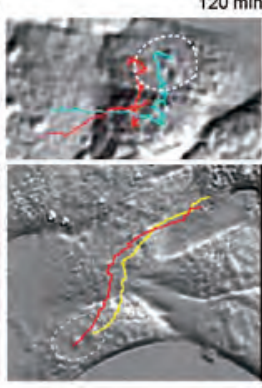

$\mathrm{C}^{\prime}$

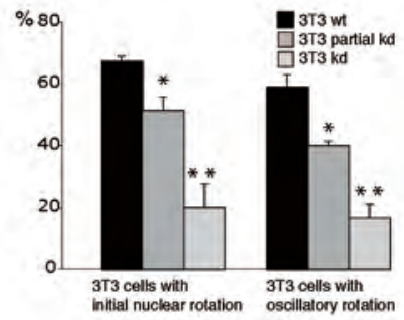

F
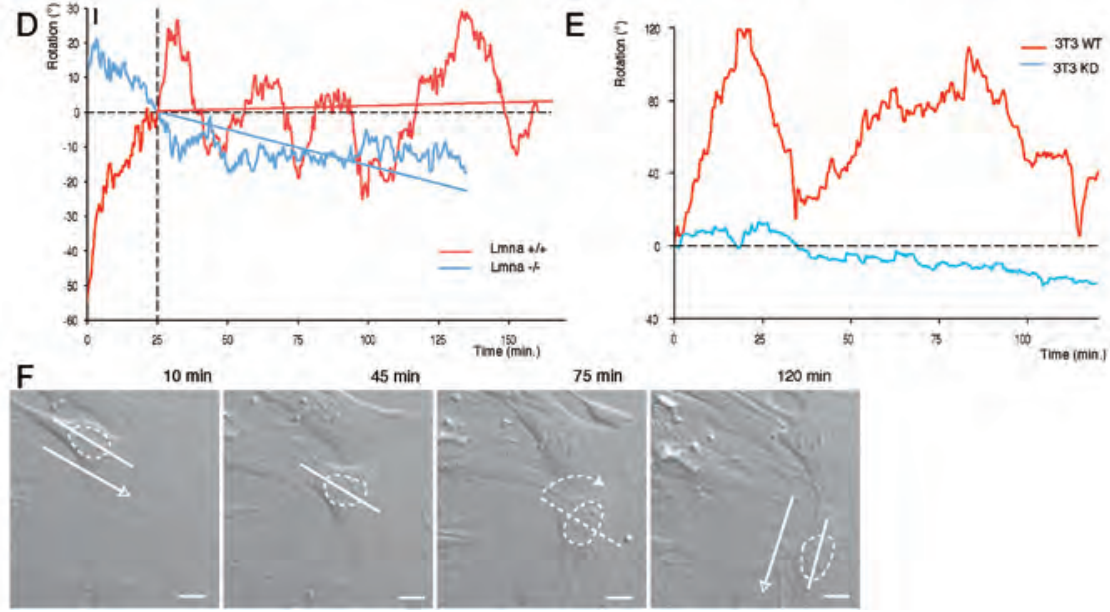
Figure 5: Nuclear reorientation and oscillatory rotation during cellular migration. Wt MEFs (A, upper panels) exhibit an initial rotation of the nucleus towards the direction of migration during cellular migration while lmna -/- MEFs (A, lower panels) do not change their nuclear orientation at the beginning of the migration. In panel $\mathrm{A}$ the direction of cell migration is denoted by a straight arrow. A curved dashed arrow denotes the rotation of the nucleus. Straight lines indicate the orientation of the nucleus. Quantitative analysis (A') of recordings of 25-30 cells in at least 4 different experiments revealed that $60 \pm 3 \%$ of wt MEFs $(n=129)$ demonstrated active repositioning of the nucleus prior to cellular migration, only $7 \pm 1 \%$ of Imna -/- MEFs $(\mathrm{n}=117)$ showed this behaviour. During migration, wt MEFs (B, upper panels) show a typical oscillatory rotation (rotation of the nucleus back and forth) of the nucleus, absent in lmna -/- MEFs (B, lower panels). Quantitative analysis (B') of recordings of 25-30 cells in at least 4 different experiments showed that $66 \pm 3 \%$ of wt MEFs $(n=129)$ demonstrated oscillatory rotation of the nucleus during cellular migration, while only $9 \pm 1 \%$ of lmna -/- MEFs $(\mathrm{n}=117)$ showed this behaviour. The knockdown models in $3 \mathrm{~T} 3$ cells demonstrate a direct coupling of the amount of lamins and the presence of nuclear oscillatory rotation. $67 \pm 2 \%$ of wt $3 \mathrm{~T} 3$ cells $(\mathrm{C}$, upper panels) demonstrated initial nuclear rotation and $59 \pm 4 \%$ of these cells showed nuclear oscillatory rotation, while only $20 \pm 8 \%$ of the $3 \mathrm{~T} 3$ lamin A/C kd cells (C, lower panels) demonstrated initial nuclear rotation and $17 \pm 1 \%$ of these cells showed nuclear oscillatory rotation. The quantitative analysis of the wt $3 T 3(n=83)$, pkd $(n=71)$ and kd cells $(n=60)$ is shown in C'. The angular rotation of representative MEF (D) and 3T3 cells (E) during the ongoing migration was plotted in time. The wt nucleus (D, E: red line) shows abrupt rotation-angle changes (oscillations), while the lmna -/nucleus (D, E: blue line) shows no abrupt rotations back and forth. Regression analysis (straight lines) shows the net rotation. Lmna -/- nuclei show a larger net rotation (drift). The rotation of the nucleus towards the direction of migration can also be observed in wt MEF cells (F) when the cells changes its direction of migration at later stages of migration (direction denoted by straight arrow). All scale bars are $10 \mu \mathrm{m}$. Single asterisks denote $\mathrm{p}<0.05$ (Student's t-test). Double asterisks denote $\mathrm{p}<0.01$ (Student's ttest).

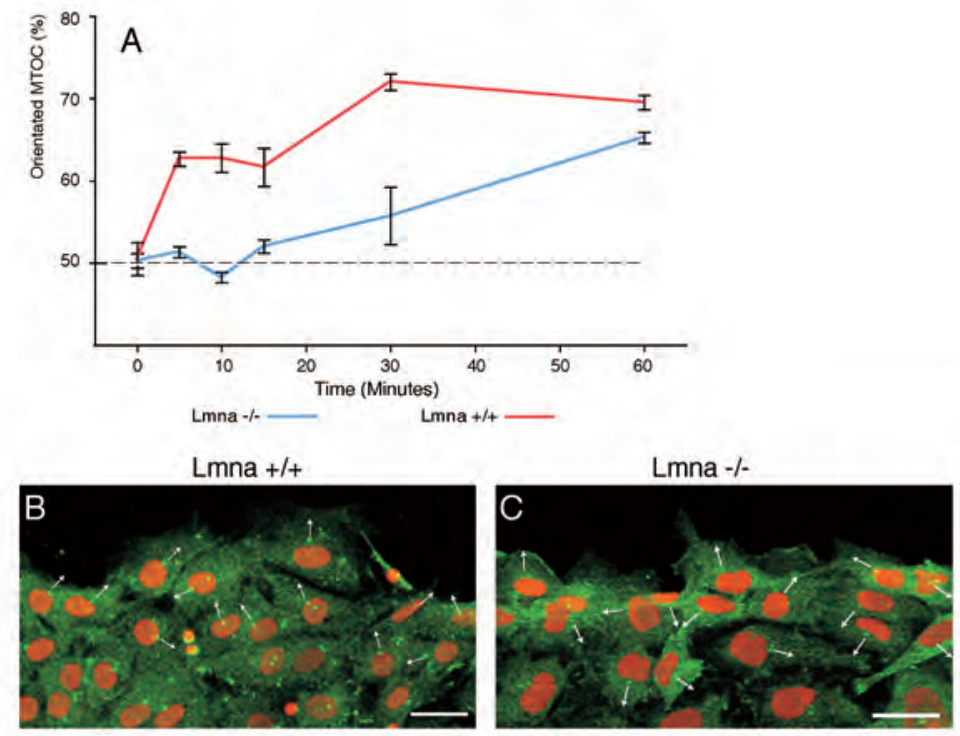

Figure 6: MTOC reorientation during cell migration. (A) Plot of percentage of cells with nuclear orientation towards wound edge versus time after wounding. At the time of wounding, the MTOC in both lmna -/- and wt MEFs are randomly organized around the nucleus, seen as 50\% of the cells with correct MTOC orientation. In wt MEFs the MTOC reorganize towards the wound edge within 30 minutes, while lmna -/cells need 60 minutes to show a maximal reorientation. Note differences in correct reorientation between wt 
(B) and lmna -/- cells (C) 30 minutes after wounding. 150 cells of either cell type at every time point were measured. Scale bars represent $50 \mu \mathrm{m}$.

\section{Alterations in cell adhesion molecules due to the absence of A-type lamins}

We compared the presence and localization of some cell membrane-associated proteins, i.e. connexin-43, dephosphorylated $\beta$-catenin, vinculin and $\mathrm{N}$-cadherin in wild type, lamin $\mathrm{A} / \mathrm{C}$-deficient and lamin $\mathrm{A} / \mathrm{C}$ kd cells using an immunofluorescence approach. No apparent differences in expression levels or localization were observed for N-cadherin, vinculin and $\beta$-catenin between wild type and lmna -/- MEFs (Fig. 7). However, connexin-43 forms discrete gap junctions at the cellular lining only in wild type MEFs (Fig. 7A), while in lmna -/- cells this typical staining pattern was not observed (Fig. 7B). No such alterations were found in the $3 \mathrm{~T} 3$ cells, since in this subline we could not detect connexin-43 localized in connexons, neither in the wild type nor in the kd cells (data not shown).

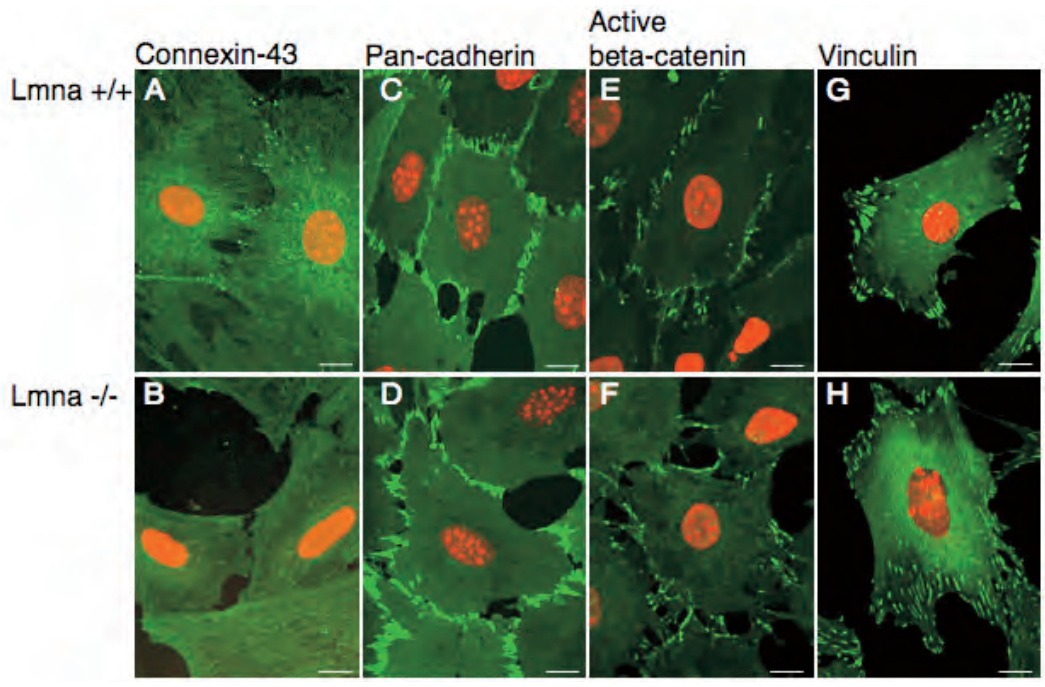

Figure 7: Distribution of adhesion molecules in wild type and lmna -/- MEFs

Distribution of connexin-43 (A, B), pan-cadherin (C, D), the active form of $\beta$-catenin $(E, F)$ and vinculin $(G, H)$ in wild type MEFs (A, C, E, G) and lmna -/- MEFs (B, D, F, H), using immunofluorescence. Connexin-43 shows a distinct labelling of gap junctions at cell-cell contacts in wild type MEFs (A). This pattern cannot be seen in lmna -/- MEFs (B). The other adhesion molecules did not show any differences between the two cell types (C-H). All scale bars are $10 \mu \mathrm{m}$. 


\section{Membrane protrusions during wound healing}

An essential part of the process of cellular migration is the formation of membrane protrusions at the leading edge of the migrating cell. At 30 minutes after wounding, it became obvious that membrane ruffles were more abundant and wider in lmna -/-
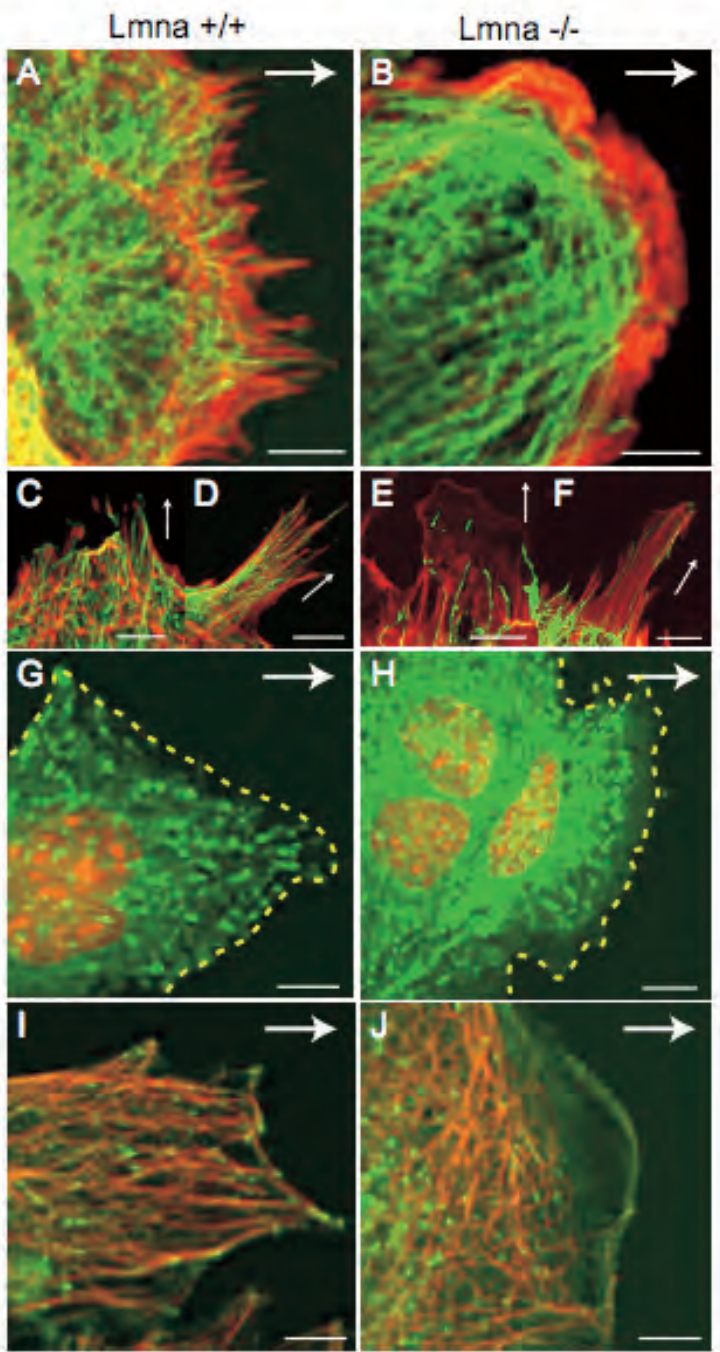

Figure 8: Membrane ruffling and filopodia formation in wound-edge. Immunofluorescent labelling of cellular borders at the wound edge (straight arrow) 30 minutes after wounding. Wild type MEFs (A, C, D, G, I) and lmna /- MEFs (B, E, F, H, J) were labelled with phalloidin (red) and $\beta$-tubulin (green) (A-F), with EB1 (green) (G, H) or with EB1 (green) and $\alpha$-tubulin (red) (I, J). Lmna -/- MEFs demonstrated more and thicker membrane ruffling (B) than wild type MEFs (A). In wild type MEFs, all membrane protrusions contained both actin filaments and microtubules (C-D), while $30 \%$ of lmna -/- MEFs had protrusions that contained only remnants of microtubules 
(E-F). EB1-staining showed a decoration of microtubules extending into the protrusions of wild type MEFs (G), while lmna -/- MEFs demonstrated regions within membrane protrusions that were devoid of EB1-staining (H). A yellow dashed line indicates the border of the cells at the wound edge. Double labelling of EB1 and microtubules show that microtubules extending into the protrusions in wild type MEFs are capped with EB1 (I), while microtubules of lmna -/- cells have much less EB1 capping especially at the protrusions (J). All scale bars are 10 $\mu \mathrm{m}$.

MEFs than in wild type MEFs (Fig. 8A, B). Double labelling for actin and tubulin revealed that about $30 \%$ of the lmna -/- cells at the leading edge of the wound demonstrated actin-containing protrusions (filopodia), which, however, to a large extent lacked microtubules (Fig. 8C-F). In contrast, wild type cells showed abundant microtubules in all filopodia. Since microtubule growth and stabilization are essential for cellular movement, and EB1 is a marker for growing microtubules [42], we determined the distribution of this capping protein at the wound edge 30 minutes after wounding. While in the wild type MEFs EB1 showed capping of microtubules throughout the cell up to the plasma membrane (Fig. 8G), lmna -/- cells demonstrated regions of cellular protrusions without any EB1 (Fig. $8 \mathrm{H}$ ), indicating that there is less stabilization of microtubules in lmna -/- cells (Fig.8I, J).

\section{Effects of lamin-deficiency on MAPK activity}

Previous studies have shown an upregulated signalling of the MAPK pathways in lamin $\mathrm{A} / \mathrm{C}$ deficient cells and in laminopathies [43-45].To confirm this for our cell lines this, we measured the amount of phospho-Erk1/2 in 3T3 kd cells, Imna -/- MEFs and corresponding wt cells after western blotting (Fig. 9). Cells were tested in 3 conditions, in growth medium (DMEM $+10 \%$ FCS), in serum starvation medium (DMEM + 0,5\% FCS) and in serum starvation medium with $100 \mathrm{ng} / \mathrm{ml}$ EGF (for 10 minutes). Actin was used as a control for the amount of protein loaded onto the gels. In wt 3 T3 cells, only minor effects of EGF were seen on the amount of phosphoErk $1 / 2$ under the different culturing conditions. As expected, serum starvation medium induced a decline in the amount of phospho-Erk1/2. EGF-stimulation showed a minor increase of phospho-Erk1/2on the MAPK signalling in these cells. The basal values (growth medium cultures) of the $3 \mathrm{~T} 3 \mathrm{kd}$ cells are comparable to the values in the $\mathrm{wt}$ $3 \mathrm{~T} 3$ cells, however, in serum starvation medium there was no decline but even a small increase in the amount of phospho-Erk1/2. The largest effect was seen after EGF stimulation, the amount of EGF showed a clear increase (Fig. 9 panel A). A prominent difference was observed between wild type MEF cells and lmna -/- MEFs For wt MEFs, a strong rise in phospho-Erk 1 and phospho-Erk 2 was seen after EGF stimulation. However, in the lmna -/- MEFs, the basal level of phospho-Erk1/2 in 
growth medium was much higher when compared to wt MEFs. Serum starvation showed only a minor decease, while stimulation with EGF had no additional positive effect on the amount of Erk1/2 in the cells (Fig 9 panel B).

A

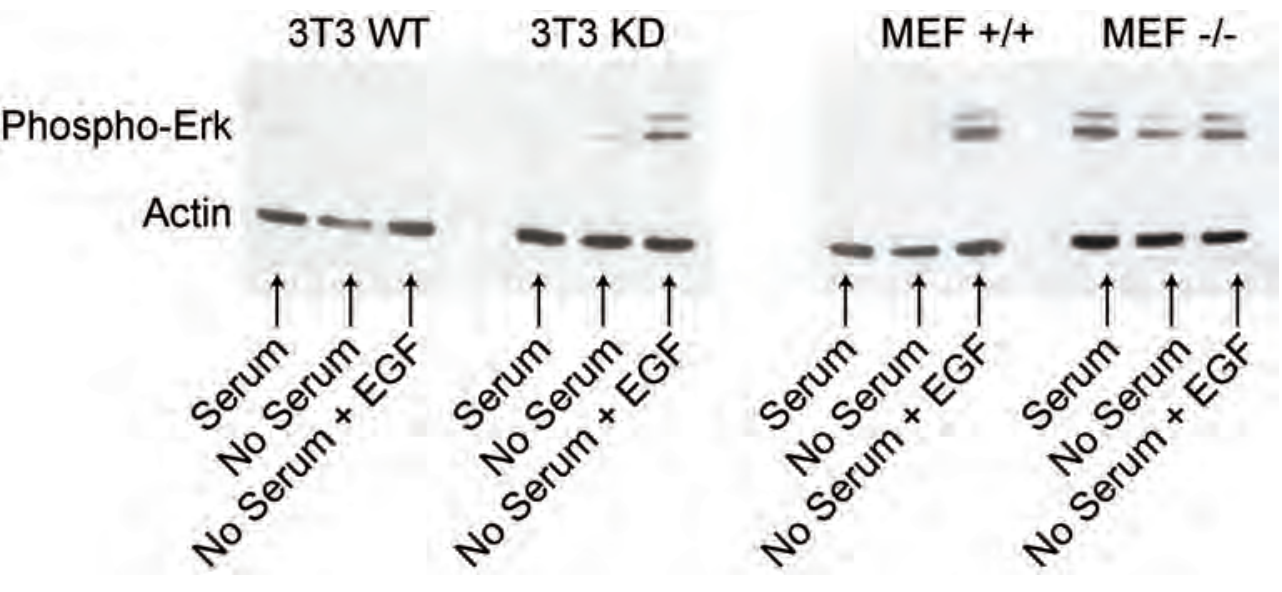

$3 T 3 \mathrm{KD}$

Phospho-Erk

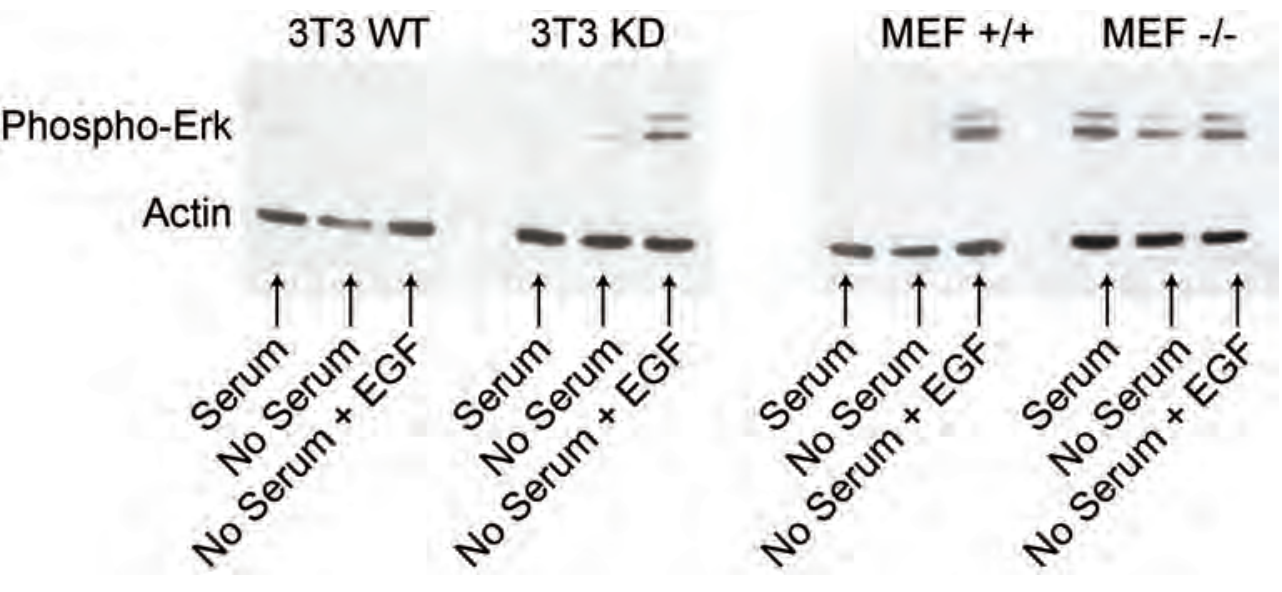

B MEF $+1+\quad$ MEF $-/-$

Figure 9: Western blot analysis using the p42/p44 (phospho-Erk1/2) antibody, Panel A: $3 \mathrm{t} 3$ cells, panel MEF cells. 3T3 KD cells demonstrate no decrease after serum starvation and a prominent increases increase of phospho-Erk1/2 after EGF stimulation, while wt 3 T3 cells showed a very weak response. wt MEF cells showed a very weak signal in control and serum starvation conditions, and a clear an increase of phospho-Erk1/2 after EGF stimulation. In contrast lmna -/- MEF cells had a constant high amount of phospho-Erk1/2, under every culturing condition. 


\section{Discussion}

The absence of A-type lamins affects the organization and function of the different elements of the nucleo-cytoskeletal scaffold. Especially, nuclear attachment of microfilaments, microtubules and intermediate filaments is disturbed, and nuclear membrane associated proteins dislocate to the endoplasmic reticulum. Here we show, that both in lmna -/- cells and in lmna kd cells these structural abnormalities are associated with disturbances in nuclear motility and cellular migration during in vitro wound healing.

\section{Nucleo-cytoskeletal changes in A-type lamin deficient cells}

It has been shown that in Imna -/- cells the integration of emerin into the nuclear membrane is disturbed and that this protein relocates to the endoplasmic reticulum [38, 46]. The impact of A- type lamin knockdown on structural alterations was confirmed by emerin localization in the $3 \mathrm{~T} 3$ cells that were stably transfected with an A-type lamin RNAi vector. Next to emerin, A-type lamins mediate the binding of nesprin-1, -2 and -3 to the nuclear membrane $[13,15,17,35,47]$, but it remains unclear whether lamins bind nesprin directly or via other binding proteins. At the cytoplasmic side of the nuclear membrane the nesprin proteins are linked to the cytoskeletal filaments, with nesprin-1 and -2 linking to actin filaments [13], while nesprin-3 binds to intermediate filaments via plectin [35]. In C. Elegans a link of the tubulin network and the MTOC to the nucleus is mediated by UNC-83 and Zyg-12, respectively [25-28],, but the mammalian homologues of these proteins have so far not been identified. Our results show a loss of nesprin-3 in the nuclear envelope of both the lmna -/- MEFs and the $3 \mathrm{~T} 3 \mathrm{kd}$ cells. Ketema et al. [48] showed a comparable partial relocalization of nesprin-3 to the endoplasmic reticulum after knockdown of SUN1 and 2, the linker proteins that connect lamin A/C to nesprin-3 [35, 48], but no obvious relocalization of nesprin-3 after a transient knockdown of lamin $\mathrm{A} / \mathrm{C}$. In both our stable knockdown models a direct correlation exists between the levels of lamin $\mathrm{A} / \mathrm{C}$ expression and the relocalization of nesprin-3 at the individual cell level, and it seems therefore likely that cells need to go through several cell division cycles before the effects of the loss of lamins become apparent. The relocalization of nesprin-3, emerin and possibly also other LINC proteins leads to a partial disconnection of the different cytoskeletal elements from the nucleus. 
Especially, local nuclear aberrations of nesprin-3 in lmna -/- cells correlate directly with distinct changes in the vimentin intermediate filament organization in connected regions of the cytoplasm.

The reduction of actin stress fibers and the absence of perinuclear actin fibers, the unequal distribution of tubulin throughout the cell and the abnormal perinuclear vimentin organization, in particular in areas where nesprins are absent in lmna -/- cells, predict that the absence of A-type lamins has a major effect on the structural integrity of the nucleo-skeletal connections. Therefore we have examined the integrity of this connection by detergent extractions of these cells. We found indeed a diminished and disorganized attachment of cytoskeletal components to lmna -/- nuclei, in particular for microfilaments and intermediate filaments, as compared to wt nuclei. Additional evidence that the coupling of the cytoskeleton to the nucleus is disturbed in lamin $\mathrm{A} / \mathrm{C}$ deficient cells comes from previous mechanical compression studies [7]. Nuclei of wt cells deform in anisotropic fashion upon compression of intact cells, and isotropically after detergent extraction. In contrast, the nuclei of lmna -/- cells already deform isotropically without extraction, indicating that these nuclei are detached from the tension that is exerted upon them by the cytoskeleton [9]. When findings in lmna -/MEFs are compared to those of lamin A/C $3 \mathrm{~T} 3 \mathrm{kd}$ cells, comparable changes in MTOC distance to the nucleus are found. In both systems the distance between the nucleus and the MTOC is almost doubled in the absence of A-type lamins. It is therefore obvious that downregulation of A-type lamins affects the cytoskeletal organization.

\section{Disturbed reorientation of the nucleus and the MTOC}

We anticipated that the observed structural changes in the nucleo-cytoskeletal scaffold affect normal mechanical functioning of the cell. It has already been shown that lmna /- cells are more vulnerable than wt cells when subjected to external mechanical stress $[4,7]$.

We tested the functionality of the lmna - /- MEF and $3 \mathrm{~T} 3 \mathrm{kd}$ cells in the in vitro wound healing assay. In this assay several basic aspects of cellular behaviour can be examined simultaneously, including cellular stress response, polarization, reorientation and migration [49]. The confluent monolayers of wt MEFs typically reacted to wounding by cell polarization towards the wound edge in the first phase, followed by the formation of membrane protrusions and the reorientation of the nucleus and the MTOC. Cells migrated into the wound area with simultaneous induction of mitosis. This response requires a concerted action of all cytoskeletal components. While elongation and migration of cells is associated with the formation of membrane ruffles, 
filopodia and lamellipodia, the direction of motion is partly controlled by (re) orientation of the nucleus and the MTOC [23]. Lmna -/- MEFs only partly showed this response. Upon wounding a prominent migration of these cells was seen, indicating that the affected cytoskeletal components, including actin-myosin interactions, were at least partially functioning. It has been described [23] that nuclear movement is driven by actin retrograde flow and that this process is myosin II dependent. This process is determined by the structural link between the nucleus and the (actin) cytoskeleton. Since this structural link is absent/aberrant in lmna -/- cells, it indeed seems likely that the direct effect of this uncoupling of actin from the nucleus is more important than possible actin-myosin abnormalities. Since the mobility of the lmna -/- cells is not affected, this indicates that this part of the actin-myosin mechanism is still largely intact. As documented previously [23, 24, 50-52], we observe that wt MEFs rotate their nucleus, including the MTOC, towards the wound edge prior to repolarization and movement in the migrational direction [53]. In contrast, Imna -/- cells were found to have delayed MTOC reorientation during the initiation of migration into the wound. These findings are partially in accordance to those of Lee et al. [8], but these authors describe a complete lack of MTOC reorientation up to 3 hours after wounding, while our study shows that the reorientation does take place in most cells within 60 minutes after wounding. We hypothesize that the lack of an initial MTOC reorientation response in lmna -/- cells may be a consequence of the loss of a direct link of lamins with the nucleus via Hook-like proteins [29, 30] or nesprins [33] to the MTOC, which is supported by the increase in distance of the MTOC to the nucleus in lamin depleted cells [8]. Similar observations on the MTOC distance to the nucleus were made in an emerin knockdown model [54]. The indirect connection of the MTOC to the nucleus via microtubules $[29,30]$ then causes its delayed reorientation. In this phase of the wound healing process, after the cell has determined the direction of migration, small oscillatory rotations of the nucleus as observed in the wt MEFs are needed to constantly correct the orientation of the nucleus towards the wound edge. In contrast, such correctional rotations are absent in the majority of lmna -/- MEFs, resulting in a drift of the nuclear orientation after prolonged migration. Again, the disturbed direct link of the MTOC to the nucleus and the diminished interactions with cytoskeletal filaments may explain the absence of these small oscillatory movements of the nucleus. The necessity of A-type lamins for a correct nuclear behaviour during wound healing becomes even more evident in the $3 \mathrm{~T} 3$ cells with variable levels of A-type lamin knockdown. A direct correlation between the amount of lamin $\mathrm{A} / \mathrm{C}$ present in the cells and the occurrence and extent of nuclear rotations was seen in this system. Cells with the lowest levels of A-type lamins showed the lowest degree of nuclear reorientation. 


\section{Functional changes at the cellular periphery}

The formation of membrane protrusions like filopodia and ruffles is a key factor in the process of cellular migration and needs the rearrangement of the actin and tubulin cytoskeleton, while a complex signalling cascade of Rho-GTPases coordinates these processes. In lmna -/- cells, the formation of the actin- and tubulin-dependent protrusions is disturbed. Normally, microtubules grow towards the leading edge of the migrating cell [18], as illustrated by the EB1 capping proteins, which are localized at the plus-ends of growing microtubules $[55,56]$. Here, we show that $30 \%$ of the migrating lmna -/- MEFs have strikingly diminished numbers of microtubules extending into the membrane protrusions. Since also EB1 capping appears to be largely absent in these extensions, it is concluded that stabilization of microtubules at the leading edge is diminished in lmna -/- cells. Strikingly, these aberrations in the organization of actin and tubulin at the leading edge do not prevent the migration of lmna -/- cells into the wound area. In fact, these cells apparently move faster than wild type cells. However, the direction of migration does not seem to be controlled by surrounding cells: cells move into one particular direction and there seems to be no correction of direction during movement. Possibly, this can be explained by the absence of connexons, The attachment of microtubules to the adhesion complexes at the plasma membrane, which occurs through EB1 and other adhesion complex molecules such as $\beta$-catenin, is essential for the correct localization of connexin-43 to the plasma membrane [57]. This can provide a plausible explanation for the observed absence of connexin-43 at the cellular membrane in lmna -/- MEFs, The loss of connexin-43 in lmna -/- cells can also explain their higher migrational speed. Similar results were described by Mori et al. [58], who showed that the downregulation of connexin-43 leads to an enhanced migration of fibroblasts,

The absence of a direct coupling of microtubules via connexons to the cellular membrane could also cause a reduced mechanical strength of lmna -/- cells, as based on a mechanical and structural cytoskeleton continuity proposed by Ingber [59, 60]. Absence of connexons has also been described by Mounkes et al. [37], demonstrating a loss of visible gap junctions in cardiomyocytes of mice with a lmna-N195K mutation. Among the laminopathies, several conduction pathologies have been documented (for a review, see [5]), which can be explained by a reduction or absence of connexin-43 in the cell-cell contacts between cardiomyocytes [37, 61, 62]. Apparently, in the heart a mutation in lamin $\mathrm{A} / \mathrm{C}$ is sufficient to cause an effect on connexin-43 transport to the membrane. 


\section{Change in the phosphorylation-status of Erk1/2}

Next to a mechanical response, our data regarding the amount of activated MAPKs indicate that a loss or decrease of lamin can induce changes in MAPK signalling response in these cells. Especially in Imna -/- MEFs a constant increase in active ERK1/2 is observed, levels that barely change under different cultures conditions that normally decrease (serum starvation) or increase (EGF stimulation) phosphorylation levels. These findings correspond to research by Emerson et al. [63] who showed an initial lack of Erk1/2 activation in lamin mutant cells. However, this was followed by hyper-activation of Erk1/2 at later time points. This hyper-activation, to some extent seen in the 3T3 kd cells could be lacking from the lmna -/- cell data, since our samples were only taken at one time point (10 minutes after stimulation). Our data of the lamin $\mathrm{A} / \mathrm{C}$ knock-down versus knockout cells show that the activation of MAPKs is stronger when no lamin $\mathrm{A} / \mathrm{C}$ is present compared to the effects seen in knock-down cells. This suggests a direct correlation between the amount of lamin A/C present in the cells and activated MAPKs.

Research from Muchir $[64,65]$ showed that upregulation of the MAPK pathway could also be seen in the hearts of $L m n a^{\mathrm{H} 222 \mathrm{P} / \mathrm{H} 222 \mathrm{P}}$ mice, leading to cardiomyopathies in these mice. Induced activation of Erk in mice also leads to hyperthrophy of the heart [66], thus leading to the conclusion that an activation of MAPKs due to a decreased amount of lamin $\mathrm{A} / \mathrm{C}$ could be a key element in the induction of certain laminopathies.

All in all, these results indicate that nuclear reorientation and the resulting influence on the cytoskeleton through interactions between the cytoplasmic scaffold and A-type lamins play critical role in the coordination of cell migration. 


\section{Acknowledgements}

The authors wish to thank Dr. B. Burke (University of Florida, Florida, USA) and Dr. C. Stewart (National Cancer Institute, Maryland, USA) for the wt and lmna -/- mouse embryonic fibroblast cell lines, Dr. G. Krohne (Würzburg, Germany) for the X-67 antibody to lamin A/C, Dr. A. Sonnenberg (Netherlands Cancer Institute, Amsterdam, the Netherlands) for the antibody to nesprin-3, Dr. C. Viebahn (University of Göttingen, Germany) for the BV-1118 antibody to vimentin and Dr. M. Borgers (Janssen Farmaceutica, Beerse, Belgium) for the antibody to $\alpha$-tubulin. The E7 monoclonal ß-tubulin antibody developed by M. Klymkowsky was obtained from the Developmental Studies Hybridoma Bank developed under the auspices of the NICHD and maintained by The University of Iowa, Department of Biological Sciences, Iowa City, IA 52242. The microscopic equipment for the vital imaging studies was financially supported by a grant from the Netherlands Organization for Scientific Research (NWO Grant 901-28-134).

\section{Video}

Video 1:

http://dl.dropbox.com/u/37827993/Chapter2-video1.mov

Video 2:

http://dl.dropbox.com/u/37827993/Chapter2-video2.mov

Video 3:

http://dl.dropbox.com/u/37827993/Chapter2-video3.mov

Video 4:

http://dl.dropbox.com/u/37827993/Chapter2-video4.mov

Video 5:

http://dl.dropbox.com/u/37827993/Chapter2-video5.mov 


\section{References}

[1] D.A. Starr and M. Han, ANChors away: an actin based mechanism of nuclear positioning, J Cell Sci 116 (2003) 211-216.

[2] M. Cohen, K.K. Lee, K.L. Wilson and Y. Gruenbaum, Transcriptional repression, apoptosis, human disease and the functional evolution of the nuclear lamina, Trends Biochem Sci 26 (2001) 41-47.

[3] D. Dorner, J. Gotzmann and R. Foisner, Nucleoplasmic lamins and their interaction partners, LAP2alpha, Rb, and BAF, in transcriptional regulation, Febs J 274 (2007) 1362-1373.

[4] J. Lammerding, P.C. Schulze, T. Takahashi, S. Kozlov, T. Sullivan, R.D. Kamm, C.L. Stewart and R.T. Lee, Lamin A/C deficiency causes defective nuclear mechanics and mechanotransduction, J Clin Invest 113 (2004) 370378.

[5] J.L. Broers, F.C. Ramaekers, G. Bonne, R.B. Yaou and C.J. Hutchison, Nuclear lamins: laminopathies and their role in premature ageing, Physiol Rev 86 (2006) 967-1008.

[6] M. Crisp, Q. Liu, K. Roux, J.B. Rattner, C. Shanahan, B. Burke, P.D. Stahl and D. Hodzic, Coupling of the nucleus and cytoplasm: role of the LINC complex, J Cell Biol 172 (2006) 41-53.

[7] J.L. Broers, E.A. Peeters, H.J. Kuijpers, J. Endert, C.V. Bouten, C.W. Oomens, F.P. Baaijens and F.C. Ramaekers, Decreased mechanical stiffness in LMNA-/- cells is caused by defective nucleo-cytoskeletal integrity: implications for the development of laminopathies, Hum Mol Genet 13 (2004) 2567-2580.

[8] J.S. Lee, C.M. Hale, P. Panorchan, S.B. Khatau, J.P. George, Y. Tseng, C.L. Stewart, D. Hodzic and D. Wirtz, Nuclear lamin A/C deficiency induces defects in cell mechanics, polarization, and migration, Biophys J 93 (2007) 2542-2552.

[9] F. Houben, F.C. Ramaekers, L.H. Snoeckx and J.L. Broers, Role of nuclear lamina-cytoskeleton interactions in the maintenance of cellular strength, Biochim Biophys Acta 1773 (2007) 675-686.

[10] T.P. Kole, Y. Tseng, I. Jiang, J.L. Katz and D. Wirtz, Intracellular mechanics of migrating fibroblasts, Mol Biol Cell 16 (2005) 328-338.

[11] C.D. Nobes and A. Hall, Rho GTPases control polarity, protrusion, and adhesion during cell movement, J Cell Biol 144 (1999) 1235-1244.

[12] Q. Zhang, C. Ragnauth, M.J. Greener, C.M. Shanahan and R.G. Roberts, The nesprins are giant actin-binding proteins, orthologous to Drosophila melanogaster muscle protein MSP-300, Genomics 80 (2002) 473-481.

[13] Q. Zhang, C.D. Ragnauth, J.N. Skepper, N.F. Worth, D.T. Warren, R.G. Roberts, P.L. Weissberg, J.A. Ellis and C.M. Shanahan, Nesprin-2 is a multi- 
isomeric protein that binds lamin and emerin at the nuclear envelope and forms a subcellular network in skeletal muscle, J Cell Sci 118 (2005) 673-687.

[14] Q. Zhang, J.N. Skepper, F. Yang, J.D. Davies, L. Hegyi, R.G. Roberts, P.L. Weissberg, J.A. Ellis and C.M. Shanahan, Nesprins: a novel family of spectrin-repeat-containing proteins that localize to the nuclear membrane in multiple tissues, J Cell Sci 114 (2001) 4485-4498.

[15] T. Libotte, H. Zaim, S. Abraham, V.C. Padmakumar, M. Schneider, W. Lu, M. Munck, C. Hutchison, M. Wehnert, B. Fahrenkrog, U. Sauder, U. Aebi, A.A. Noegel and I. Karakesisoglou, Lamin A/C-dependent localization of Nesprin-2, a giant scaffolder at the nuclear envelope, Mol Biol Cell 16 (2005) 3411-3424.

[16] V.C. Padmakumar, S. Abraham, S. Braune, A.A. Noegel, B. Tunggal, I. Karakesisoglou and E. Korenbaum, Enaptin, a giant actin-binding protein, is an element of the nuclear membrane and the actin cytoskeleton, Exp Cell Res 295 (2004) 330-339.

[17] V.C. Padmakumar, T. Libotte, W. Lu, H. Zaim, S. Abraham, A.A. Noegel, J. Gotzmann, R. Foisner and I. Karakesisoglou, The inner nuclear membrane protein Sun1 mediates the anchorage of Nesprin-2 to the nuclear envelope, $\mathrm{J}$ Cell Sci 118 (2005) 3419-3430.

[18] G.G. Gundersen and J.C. Bulinski, Selective stabilization of microtubules oriented toward the direction of cell migration, Proc Natl Acad Sci U S A 85 (1988) 5946-5950.

[19] A.F. Palazzo, T.A. Cook, A.S. Alberts and G.G. Gundersen, mDia mediates Rho-regulated formation and orientation of stable microtubules, Nat Cell Biol 3 (2001) 723-729.

[20] A.F. Palazzo, C.H. Eng, D.D. Schlaepfer, E.E. Marcantonio and G.G. Gundersen, Localized stabilization of microtubules by integrin- and FAKfacilitated Rho signaling, Science 303 (2004) 836-839.

[21] Y. Mimori-Kiyosue, I. Grigoriev, G. Lansbergen, H. Sasaki, C. Matsui, F. Severin, N. Galjart, F. Grosveld, I. Vorobjev, S. Tsukita and A. Akhmanova, CLASP1 and CLASP2 bind to EB1 and regulate microtubule plus-end dynamics at the cell cortex, J Cell Biol 168 (2005) 141-153.

[22] Y. Mimori-Kiyosue, N. Shiina and S. Tsukita, The dynamic behavior of the APC-binding protein EB1 on the distal ends of microtubules, Curr Biol 10 (2000) 865-868.

[23] E.R. Gomes, S. Jani and G.G. Gundersen, Nuclear movement regulated by Cdc42, MRCK, myosin, and actin flow establishes MTOC polarization in migrating cells, Cell 121 (2005) 451-463.

[24] J.S. Lee, M.I. Chang, Y. Tseng and D. Wirtz, Cdc42 mediates nucleus movement and MTOC polarization in Swiss 3 T3 fibroblasts under mechanical shear stress, Mol Biol Cell 16 (2005) 871-880. 
[25] D.A. Starr and M. Han, A genetic approach to study the role of nuclear envelope components in nuclear positioning, Novartis Found Symp 264 (2005) 208-219; discussion 219-230.

[26] D.A. Starr, G.J. Hermann, C.J. Malone, W. Fixsen, J.R. Priess, H.R. Horvitz and $\mathrm{M}$. Han, unc- 83 encodes a novel component of the nuclear envelope and is essential for proper nuclear migration, Development 128 (2001) 5039-5050.

[27] M.D. McGee, R. Rillo, A.S. Anderson and D.A. Starr, UNC-83 IS a KASH protein required for nuclear migration and is recruited to the outer nuclear membrane by a physical interaction with the SUN protein UNC-84, Mol Biol Cell 17 (2006) 1790-1801.

[28] K.K. Lee, D. Starr, M. Cohen, J. Liu, M. Han, K.L. Wilson and Y. Gruenbaum, Lamin-dependent localization of UNC-84, a protein required for nuclear migration in Caenorhabditis elegans, Mol Biol Cell 13 (2002) 892901.

[29] C.J. Malone, L. Misner, N. Le Bot, M.C. Tsai, J.M. Campbell, J. Ahringer and J.G. White, The C. elegans hook protein, ZYG-12, mediates the essential attachment between the centrosome and nucleus, Cell 115 (2003) 825-836.

[30] P. Gonczy, Centrosomes: hooked on the nucleus, Curr Biol 14 (2004) R268270.

[31] K. Patterson, A.B. Molofsky, C. Robinson, S. Acosta, C. Cater and J.A. Fischer, The functions of Klarsicht and nuclear lamin in developmentally regulated nuclear migrations of photoreceptor cells in the Drosophila eye, Mol Biol Cell 15 (2004) 600-610.

[32] Y. Gruenbaum, A. Margalit, R.D. Goldman, D.K. Shumaker and K.L. Wilson, The nuclear lamina comes of age, Nat Rev Mol Cell Biol 6 (2005) 21-31.

[33] K. Wilhelmsen, M. Ketema, H. Truong and A. Sonnenberg, KASH-domain proteins in nuclear migration, anchorage and other processes, J Cell Sci 119 (2006) 5021-5029.

[34] V. Nikolova, C. Leimena, A.C. McMahon, J.C. Tan, S. Chandar, D. Jogia, S.H. Kesteven, J. Michalicek, R. Otway, F. Verheyen, S. Rainer, C.L. Stewart, D. Martin, M.P. Feneley and D. Fatkin, Defects in nuclear structure and function promote dilated cardiomyopathy in lamin A/C-deficient mice, J Clin Invest 113 (2004) 357-369.

[35] K. Wilhelmsen, S.H. Litjens, I. Kuikman, N. Tshimbalanga, H. Janssen, I. van den Bout, K. Raymond and A. Sonnenberg, Nesprin-3, a novel outer nuclear membrane protein, associates with the cytoskeletal linker protein plectin, $\mathrm{J}$ Cell Biol 171 (2005) 799-810.

[36] Y.W. Chu, R.B. Runyan, R.G. Oshima and M.J. Hendrix, Expression of complete keratin filaments in mouse $\mathrm{L}$ cells augments cell migration and invasion, Proc Natl Acad Sci U S A 90 (1993) 4261-4265.

[37] L.C. Mounkes, S.V. Kozlov, J.N. Rottman and C.L. Stewart, Expression of an LMNA-N195K variant of A-type lamins results in cardiac conduction defects and death in mice, Hum Mol Genet 14 (2005) 2167-2180. 
[38] T. Sullivan, D. Escalante-Alcade, H. Bhatt, M. Anver, N. Bhat, K. Nagashima, C.L. Stewart and B. Burke, Loss of A-type lamin expression compromises nuclear envelope integrity leading to muscular dystrophy, Journal of Cell Science 147 (1999).

[39] B.A. Kudlow, S.A. Jameson and B.K. Kennedy, HIV protease inhibitors block adipocyte differentiation independently of lamin A/C, Aids 19 (2005) 15651573.

[40] J.L. Broers, H.J. Kuijpers, C. Ostlund, H.J. Worman, J. Endert and F.C. Ramaekers, Both lamin A and lamin C mutations cause lamina instability as well as loss of internal nuclear lamin organization, Exp Cell Res 304 (2005) 582-592.

[41] B.M. Machiels, J.L. Broers, Y. Raymond, L. de Ley, H.J. Kuijpers, N.E. Caberg and F.C. Ramaekers, Abnormal A-type lamin organization in a human lung carcinoma cell line, Eur J Cell Biol 67 (1995) 328-335.

[42] K.J. Salaycik, C.J. Fagerstrom, K. Murthy, U.S. Tulu and P. Wadsworth, Quantification of microtubule nucleation, growth and dynamics in woundedge cells, J Cell Sci 118 (2005) 4113-4122.

[43] C. Favreau, E. Delbarre, J.C. Courvalin and B. Buendia, Differentiation of C2C12 myoblasts expressing lamin A mutated at a site responsible for EmeryDreifuss muscular dystrophy is improved by inhibition of the MEK-ERK pathway and stimulation of the PI3-kinase pathway, Exp Cell Res 314 (2008) 1392-1405.

[44] A. Muchir, W. Wu and H.J. Worman, Reduced expression of A-type lamins and emerin activates extracellular signal-regulated kinase in cultured cells, Biochim Biophys Acta 1792 (2009) 75-81.

[45] A. Muchir, J. Shan, G. Bonne, S.E. Lehnart and H.J. Worman, Inhibition of extracellular signal-regulated kinase signaling to prevent cardiomyopathy caused by mutation in the gene encoding A-type lamins, Hum Mol Genet 18 (2009) 241-247.

[46] A. Muchir, B.G. van Engelen, M. Lammens, J.M. Mislow, E. McNally, K. Schwartz and G. Bonne, Nuclear envelope alterations in fibroblasts from LGMD1B patients carrying nonsense Y259X heterozygous or homozygous mutation in lamin A/C gene, Exp Cell Res 291 (2003) 352-362.

[47] J.M. Mislow, J.M. Holaska, M.S. Kim, K.K. Lee, M. Segura-Totten, K.L. Wilson and E.M. McNally, Nesprin-1alpha self-associates and binds directly to emerin and lamin A in vitro, FEBS Lett 525 (2002) 135-140.

[48] M. Ketema, K. Wilhelmsen, I. Kuikman, H. Janssen, D. Hodzic and A. Sonnenberg, Requirements for the localization of nesprin-3 at the nuclear envelope and its interaction with plectin, J Cell Sci 120 (2007) 3384-3394.

[49] M. Fukata, M. Nakagawa and K. Kaibuchi, Roles of Rho-family GTPases in cell polarisation and directional migration, Curr Opin Cell Biol 15 (2003) 590597. 
[50] J. Peng, B.J. Wallar, A. Flanders, P.J. Swiatek and A.S. Alberts, Disruption of the Diaphanous-related formin Drfl gene encoding mDial reveals a role for Drf3 as an effector for Cdc42, Curr Biol 13 (2003) 534-545.

[51] C.H. Eng, T.M. Huckaba and G.G. Gundersen, The formin mDia regulates GSK3beta through novel PKCs to promote microtubule stabilization but not MTOC reorientation in migrating fibroblasts, Mol Biol Cell 17 (2006) 50045016.

[52] S. Etienne-Manneville and A. Hall, Rho GTPases in cell biology, Nature 420 (2002) 629-635.

[53] B. Szabo, Z. Kornyei, J. Zach, D. Selmeczi, G. Csucs, A. Czirok and T. Vicsek, Auto-reverse nuclear migration in bipolar mammalian cells on micropatterned surfaces, Cell Motil Cytoskeleton 59 (2004) 38-49.

[54] G. Salpingidou, A. Smertenko, I. Hausmanowa-Petrucewicz, P.J. Hussey and C.J. Hutchison, A novel role for the nuclear membrane protein emerin in association of the centrosome to the outer nuclear membrane, J Cell Biol 178 (2007) 897-904.

[55] A.S. Infante, M.S. Stein, Y. Zhai, G.G. Borisy and G.G. Gundersen, Detyrosinated (Glu) microtubules are stabilized by an ATP-sensitive plus-end cap, J Cell Sci 113 ( Pt 22) (2000) 3907-3919.

[56] Y. Wen, C.H. Eng, J. Schmoranzer, N. Cabrera-Poch, E.J. Morris, M. Chen, B.J. Wallar, A.S. Alberts and G.G. Gundersen, EB1 and APC bind to mDia to stabilize microtubules downstream of Rho and promote cell migration, Nat Cell Biol 6 (2004) 820-830.

[57] R.M. Shaw, A.J. Fay, M.A. Puthenveedu, M. von Zastrow, Y.N. Jan and L.Y. Jan, Microtubule plus-end-tracking proteins target gap junctions directly from the cell interior to adherens junctions, Cell 128 (2007) 547-560.

[58] R. Mori, K.T. Power, C.M. Wang, P. Martin and D.L. Becker, Acute downregulation of connexin 43 at wound sites leads to a reduced inflammatory response, enhanced keratinocyte proliferation and wound fibroblast migration, J Cell Sci 119 (2006) 5193-5203.

[59] D.E. Ingber, Tensegrity II. How structural networks influence cellular information processing networks, J Cell Sci 116 (2003) 1397-1408.

[60] D.E. Ingber, Tensegrity I. Cell structure and hierarchical systems biology, J Cell Sci 116 (2003) 1157-1173.

[61] S. Kirchhoff, J.S. Kim, A. Hagendorff, E. Thonnissen, O. Kruger, W.H. Lamers and $\mathrm{K}$. Willecke, Abnormal cardiac conduction and morphogenesis in connexin40 and connexin43 double-deficient mice, Circ Res 87 (2000) 399405.

[62] D.E. Gutstein, G.E. Morley, H. Tamaddon, D. Vaidya, M.D. Schneider, J. Chen, K.R. Chien, H. Stuhlmann and G.I. Fishman, Conduction slowing and sudden arrhythmic death in mice with cardiac-restricted inactivation of connexin43, Circ Res 88 (2001) 333-339. 
[63] L.J. Emerson, M.R. Holt, M.A. Wheeler, M. Wehnert, M. Parsons and J.A. Ellis, Defects in cell spreading and ERK1/2 activation in fibroblasts with lamin A/C mutations, Biochim Biophys Acta 1792 (2009) 810-821.

[64] A. Muchir, P. Pavlidis, G. Bonne, Y.K. Hayashi and H.J. Worman, Activation of MAPK in hearts of EMD null mice: similarities between mouse models of $\mathrm{X}$-linked and autosomal dominant Emery Dreifuss muscular dystrophy, Hum Mol Genet 16 (2007) 1884-1895.

[65] A. Muchir, P. Pavlidis, V. Decostre, A.J. Herron, T. Arimura, G. Bonne and H.J. Worman, Activation of MAPK pathways links LMNA mutations to cardiomyopathy in Emery-Dreifuss muscular dystrophy, J Clin Invest 117 (2007) 1282-1293.

[66] O.F. Bueno and J.D. Molkentin, Involvement of extracellular signal-regulated kinases 1/2 in cardiac hypertrophy and cell death, Circ Res 91 (2002) 776-781. 


\section{Chapter 3}

\section{Increased plasticity of the nuclear envelope and hypermobility of telomeres due to the loss of A-type lamins}

W.H. De Vos, F. Houben, R.A. Hoebe, R. Hennekam, B. van Engelen, E.M.M. Manders, F.C.S. Ramaekers, J.L.V. Broers, P. Van Oostveldt

Published in part in:

Biochimica Biophysica Acta - General Subjects; 1800 (2010) 448-458 


\section{Abstract}

The nuclear lamina provides structural support to the nucleus and has a central role in defining nuclear organization. Defects in its filamentous constituents, the lamins, lead to a class of diseases collectively referred to as laminopathies. On the cellular level, lamin mutations affect the physical integrity of nuclei and nucleo-cytoskeletal interactions, resulting in increased susceptibility to mechanical stress and altered gene expression. In this study we quantitatively compared nuclear deformation and chromatin mobility in fibroblasts from a homozygous nonsense LMNA mutation patient and a Hutchinson-Gilford progeria syndrome patient with wild type dermal fibroblasts, based on the visualization of mCitrine labeled telomere binding protein TRF2 with light-economical imaging techniques and cytometric analyses. Without application of external forces, we found that the absence of functional lamin A/C leads to increased nuclear plasticity on the hour and minute time scale but also to increased intranuclear mobility down to the second time scale. In contrast, progeria cells show overall reduced nuclear dynamics. Experimental manipulation (farnesyltransferase inhibition or lamin $\mathrm{A} / \mathrm{C}$ silencing) confirmed that these changes in mobility are caused by abnormal or reduced lamin $\mathrm{A} / \mathrm{C}$ expression. These observations demonstrate that $\mathrm{A}$ type lamins affect both nuclear membrane and telomere dynamics. Because of the pivotal role of dynamics in nuclear function, these differences likely contribute to or represent novel mechanisms in laminopathy development. 


\section{Introduction}

The human cell nucleus is a highly organized architectural entity with chromatin as well as proteins and RNA occupying distinct and functionally relevant nuclear domains, jointly confined by the boundary of the nuclear envelope [1-3]. Proximal to the inner nuclear membrane, lays an intimately connected meshwork composed of intermediate filaments, termed lamins. Two classes of lamins can be distinguished by whether they remain associated with membrane vesicles during mitosis (B-type) or not (A-type). Both types of lamins form separate but interacting microdomains in the lamina [4] and are also found within the nuclear interior and in transnuclear tube-like structures $[5,6]$.

The lamina provides structural support to the nucleus and has a central role in defining interphase nuclear organization [7]. Defects in nuclear architecture can contribute to or directly cause human disease. Mutations in genes encoding nuclear envelope (NE) associated proteins lead to a broad spectrum of nuclear envelopathies, the most striking class of which are the laminopathies [8-10]. This diverse cluster of diseases is caused by mutations in the LMNA gene or genes encoding proteins that affect its end products' (lamin A/C) posttranslational processing. Mutations in these genes give rise to muscular dystrophies, lipid dystrophies, neuropathies, and the premature-ageing syndromes Restrictive Dermopathy and Hutchinson-Gilford progeria syndrome (HGPS) [11, 12]. These progeroid disorders arise from a defective maturation of the lamin A precursor, prelamin A. In normal healthy cells, prelamin A undergoes a 4-step process of posttranslational modifications consisting of farnesylation and methylation of the terminal cysteine and double cleavage by ZMPSTE24 endoprotease [8, 13]. In HGPS, the activation of a cryptic splice site generates a truncated form of prelamin A, referred to as progerin, that lacks the endoproteolytic cleavage site needed for correct lamin A maturation. Accumulation of progerin is toxic to cells [14] and results in progressive deformation (blebbing, herniations, invaginations) of the nuclear rim [15, 16]. The causative mechanisms of disease in laminopathies are not yet completely understood, but in general lamin defects alter the physical integrity of nuclei, resulting in increased susceptibility to mechanical stress and alterations in (mechanosensitive) gene expression [17-20]. Nuclei from lmna -/- mice show decreased mechanical stiffness [21, 22], whilst in HGPS cells the opposite holds true [23]. On the other hand, early passage or farnesyltransferase inhibitor (FTI) treated HGPS cells display an equally high sensitivity to mechanical stress as late passage cells, making it unlikely that nuclear fragility is the prime determinant at least for this specific laminopathy [23]. From the cytoplasm's perspective, the nuclear lamina anchors the nucleus to the 
cytoskeleton via components of the NE-bridging LINC complex [24]. During interphase such an interaction may not only be important for correct positioning of the nucleus and maintenance of cellular mechanical stiffness [25-27], but may also cater for the appropriate transmission of mechanically induced signalling from the cell surface to the nucleus [28].

Disruption of the nuclear lamina also directly affects chromatin organization and gene expression [29]. Heterochromatin is omnipresent at the nuclear periphery and repositioning of certain genes to the nuclear lamina, induces transcriptional repression [17]. In progeria, peripheral heterochromatin is lost, which is correlated with histone methylation changes and the mislocalization of chromosomes [16, 30, 31]. The absence or down-regulation of lamin $\mathrm{A} / \mathrm{C}$ results in a decrease of heterochromatin and structural changes of chromosome territories, especially in chromosomes with high heterochromatin content $[32,33]$. Several lamina proteins are (in)directly involved in chromatin organization, replication, transcription and DNA-binding [34]. All nuclear lamins have the ability to bind to DNA in vitro [35] and large-scale mapping of genome-lamina interactions revealed over a 1000 lamina-associated domains that are characterized by low gene expression levels [36]. Several linker proteins have been identified as well, such as LBR [37] and LAP2- $\alpha$ [38]. The latter protein transiently associates with specific heterochromatic parts of chromatin, the telomeres. Interestingly, telomeres are retained after nuclear elution procedures, which gave rise to the hypothesis that these chromosomal elements are anchored to a nuclear scaffold structure [39, 40]. Recent data from Schmitt [41] and Schober [42] show that SUNproteins (Mps3, SUN2) tether telomeres to the nuclear envelope.

To determine whether lamin A processing has an influence on telomere dynamics and nuclear dynamics in general we used telomere binding fusion proteins and lighteconomical imaging techniques such as controlled light exposure microscopy (CLEM) $[43,44]$. With this approach, we quantitatively compared multilevel nuclear dynamics between human fibroblasts deficient in functional lamin A/C, wild type and HGPS cells. We thereby distinguished different levels of nuclear plasticity and different regimes of telomere mobility. 


\section{Materials \& methods}

\section{Cell culture, transfection and drug treatment}

Primary normal human dermal fibroblasts (NHDF, Promocell, Heidelberg, Germany) were used as control cells. The following patient cells were used: fibroblasts from a patient with a nonsense Y259X homozygous mutation in lamin A/C gene ( $\mathrm{HDF}^{\mathrm{mut} / \mathrm{mut}}$ ) which are completely devoid of lamin $\mathrm{A} / \mathrm{C}$ expression [40] and fibroblasts from a patient with a LMNA G608Cmutation, suffering from Hutchison-Gilford progeria syndrome (HGPS) in which progerin accumulates in the nucleus [43]. All cell lines were cultured in advanced DMEM/F12 (Gibco, Invitrogen, Merelbeke, Belgium) supplemented with $10 \%$ FCS and $1 \%$ penicillin/streptomycin/glutamin. For transfections and imaging cells were grown on collagen coated glass bottom dishes (Matek, USA) up to approximately $75 \%$ confluency. Cells were transiently transfected with the construct pmCitrine-hTRF2 (a kind gift from Prof. H. Tanke, University of Leiden, The Netherlands), or pEGFP-LaminA using Lipofectamin 2000 (Invitrogen, Merelbeke, Belgium) according to the manufacturers' instructions. For silencing experiments, cells were double transfected with pmCitrine-hTRF2 and a LMNAtargeting shRNA vector, psiRNA-h LaminA/C, or negative control vector, psiRNALUC (Invitrogen, Toulouse, France) in which the GFP marker was replaced by tagRFP (Evrogen, Moscow, Russia). Transfected cells were imaged $48 \mathrm{~h}$ after transfection. Farnesyltransferase inhibitor (FTI) experiments were carried out by incubating cells for $72 \mathrm{~h}$ with $1 \mu \mathrm{m}$ FTI-276 (Sigma-Aldrich, St. Louis, MO, USA) and subsequent recording.

\section{Immunocytochemistry}

Cells were grown on glass slides up to $75 \%$ confluency, fixed for $10 \mathrm{~min}$ in $2 \%$ formaldehyde/PBS and washed in PBST (PBS $+0.05 \%$ Tween $)\left(3^{\circ} \mathrm{C}-5 \mathrm{~min}\right)$. Cells were permeabilized in PBS containing $0.5 \%$ Triton $X$ after which they were blocked in $4 \%$ BSA (15min). Then, slides were incubated in a humid chamber at room temperature with primary antibodies for $1 \mathrm{~h}$, washed 3 times in PBST and subsequently incubated with either Alexa-555 or Alexa-488 coupled secondary antibodies (Invitrogen, USA). After 3 washes with PBS, slides were dehydrated in an ethanol series and mounted in Vectashield ${ }^{\circledR}$ containing $1 \mathrm{ng} / \mathrm{ml}$ DAPI. Primary antibodies used in this study were rabbit anti-Lamin A (ab26300, Abcam, Cambridge, UK) and rabbit anti-Lamin B (ab16048, Abcam, Cambridge, UK). 


\section{Image acquisition}

For live cell imaging, glass bottom, containing transfected cells, were filled with prewarmed medium and sealed off with Parafilm, after which they were inserted into an incubator microscope and allowed to equilibrate to $37{ }^{\circ} \mathrm{C}$ for $30 \mathrm{~min}$. Cells were observed under a Nikon C1 confocal microscope on a TE2000 Eclipse (Nikon Instruments, Badhoevedorp, The Netherlands) equipped with Hamamatsu E717-63 photomultipliers (Hamamatsu, Hamamatsu City, Japan) and custom-made CLEM electronics [44]. Experiments were carried out with a Nikon Plan Apo 40x/1.0 PH3 objective and EZ-C1 3.30 software from Nikon in combination with an in house developed time-lapse imaging software. An AOM (Isomet, Springfield, USA) was placed in the optical path of a 488-nm argon-ion laser at $25 \mathrm{~mW}$. A HeNe laser (633 $\mathrm{nm})$ was used for simultaneous phase contrast imaging. In the time-lapse experiments, 3D images were acquired every $2 \mathrm{~min}$, for 80-120 min. Images were sampled at $288 \mathrm{x}$ $288 \times 10(\mathrm{X}, \mathrm{Y}, \mathrm{Z})$ pixels. A trade-off was made between cell survival (minimal illumination), recording speed and acceptable SNR. Therefore the laser power was physically attenuated with two neutral density filters (ND8 and ND4) and the pinhole was set to a medium opening (back projected pinhole radius $=750 \mathrm{~nm}$ ). The physical voxel size was $104 \mathrm{~nm} \times 104 \mathrm{~nm}$ x $500 \mathrm{~nm}$. CLEM settings were adjusted as described before [45].

For imaging of fixed material a Nikon TE Eclipse 2000 confocal microscope was used with a $60 \mathrm{x} / 1.4$ oil plan apo objective. These images were acquired with a minimum pinhole opening (1AU) and sampled at Nyquist criteria (voxel size of $50 \mathrm{~nm} \times 50 \mathrm{~nm} \times$ $100 \mathrm{~nm}$ ). The same type of microscope was also used in widefield fluorescence mode for acquisition of immunostained cells and short-term live cell imaging. Fluorescence detection was done with an EM-CCD camera (Andor Technology, Belfast UK). For live imaging on this microscope a $60 \mathrm{x} / 1.2 \mathrm{WI}$ lens was used and a standard mercury arc lamp, attenuated by one ND filter (ND4). For short-term live cell imaging 200 images were acquired of a single focus plane at $220 \mathrm{~ms}$ intervals. Physical pixel size was $127 \mathrm{~nm}$ x $127 \mathrm{~nm}$.

\section{Image Processing}

CLEM images were deconvolved with Huygens ${ }^{\circledR}$ software (Scientific Volume Imaging BV; Hilversum, Netherlands) according to maximum likelihood estimation. Image processing is mainly executed in ImageJ freeware [46]. 
For image correlation analysis we made use of both the temporal autocorrelation and the Pearson correlation coefficient. The temporal cross correlation follows the general expression $\quad$ [47]: $\quad(\xi, \eta, \tau)=\frac{\langle\delta i(x, y, t) \delta i(x+\xi, y+\eta, t+\tau)\rangle}{\langle i\rangle_{t}\langle i\rangle_{t+\tau}}, \quad$ where $\delta i(x, y, t)=i(x, y, t)-\langle i\rangle_{t}$ is the intensity fluctuation of the image at pixel position ( $\mathrm{x}$, $\mathrm{y})$ in time point $\mathrm{t}$ and $\tau$ represents the time lag. For temporal autocorrelation analysis the spatial lag terms $\xi, \eta$ are set to zero. The Pearson correlation coefficient is expressed as [48]: $(\tau)=\frac{\sum_{x y} \delta i(x, y, t) \delta i(x, y, t+\tau)}{\sqrt{\sum_{x y} \delta i(x, y, t)^{2} \sum_{x y} \delta i(x, y, t+\tau)^{2}}}$

Analysis of shape and deformation was performed on nuclear outlines derived after automatic segmentation of sum projections of confocal z-stacks (CLEM) or raw widefield images.

The following metrics were derived: circularity $=4 \pi\left(\frac{\text { area }}{\text { perimeter }^{2}}\right)$, convexity $=\frac{\text { area }}{\text { area } a_{\text {convex hull }}}$ compactness $=\frac{\sqrt{4 \text { area } / \pi}}{\text { major half axis } \text { ellipsoid fit }_{\text {fit }}}$, bending energy $=\frac{\sum_{\text {is contour }} \text { curvature }_{i}}{\text { contour }}$ and curvature ratio $=\frac{\text { bendingenergy }_{\text {ellipsoid fit }}}{\text { bendingenergy }}$ with curvature $_{i}=\pi-\left[\arctan 2\left[\frac{y_{i+k}-y_{i}}{x_{i+k}-x_{i}}\right]-\arctan 2\left[\frac{y_{i}-y_{i-k}}{x_{i}-x_{i-k}}\right]\right]$

Telomere tracking was performed as described before [49]. In brief, nuclear motion was first compensated for by means of a combined correlation- and centroid-based registration. Telomere positions were subsequently determined with subresolution accuracy by calculating the intensity-weighted centroids of segmented telomere spots. Widefield images were pre-processed and aligned with analogous two-dimensional algorithms. Telomeres were tracked between consecutive time points using an iterative conditional nearest neighbour algorithm and tracks were visually verified and if necessary manually corrected. Mobility was expressed in terms of peak displacement 
(the maximum distance to the origin), cumulative displacement and mean square displacement (MSD), which for each time interval $\Delta t_{j}=t_{j}-t_{o}$ can be expressed as [50]:

$\operatorname{MSD}\left(\Delta t_{j}\right)=\frac{1}{n-j} \sum_{i=j}^{n}\left[\vec{r}\left(t_{i}\right)-\vec{r}\left(t_{i-j}\right)\right]^{2}$, with $\mathrm{n}$ the total number of time points and $\vec{r}$ the n-dimensional vector of the telomere centroid at time point $t$. The MSD data were fitted in Matlab ${ }^{\circledR}$, by means of a variance-weighted non-linear least squares algorithm, to the formula of anomalous diffusion according to [51]: $\operatorname{MSD}=2 \mathrm{n} \Gamma \Delta \mathrm{t}^{\alpha}$ with $\Gamma$ the generalized transport coefficient and $\alpha$ the anomalous diffusion coefficient and to the formula for corralled motion according to [52]:

$M S D=r_{c}^{2} \cdot\left[1+\frac{2 n D_{\text {slow }} \Delta t}{r_{c}^{2}}\right] \cdot\left[1-\exp \left(-\frac{2 n D_{\text {fast }} \Delta t}{r_{c}^{2}}\right)\right]+c$ with $\mathrm{D}_{\text {slow }}$ and $\mathrm{D}_{\text {fast }}$ the slow and fast diffusion coefficients and $r_{c}$ the corral radius. $n$ represents the number of dimensions in the last two formula's. The constant reflects the localization inaccuracy due to faster mobility contributions. On average, 20 telomeres (scattered throughout the nucleus) were tracked per nucleus.

\section{Statistical Analysis}

For shape descriptors, comparison of means was performed using a standard students' T-test (for normal distributions) or non-parametric Mann-Whitney's test. Tracking results, such as displacements and MSD's did not obey normal, but rather Poisson. Therefore, the data was normalized using the BoxCox transform, allowing modelling of tracking results of individual telomeres with a general linear model, with cell type as a fixed factor [53]. Because of the relatively small amount of nuclei analyzed for CLEM recordings, the term nucleus was also incorporated into the model as a random nested factor. Non-parametric post hoc tests were included to score individual differences for significance. A p-value of 0.05 was considered statistically significant, unless stated otherwise. 


\section{Results}

\section{Lamin A/C deficiency corrodes the nuclear periphery and alters nuclear shape}

We first examined phenotypic variations between the nuclei of the three cell types after fixation. Using immunofluorescent staining of the major lamina components, lamin A and lamin B, characteristic differences readily became apparent (Fig. 1). Whereas normal fibroblasts and HGPS cells were positive for both types of lamins, HDF ${ }^{\mathrm{mut} / \mathrm{mut}}$ cells were completely devoid of lamin A and showed locally reduced presence of lamin $\mathrm{B}$ at their nuclear boundary. Staining of the lamina also revealed morphological differences. Nuclear shape was dramatically altered in $\mathrm{HDF}^{\mathrm{mut} / \mathrm{mut}}$ cells. Over $75 \%$ of all $\mathrm{HDF}^{\mathrm{mut} / \mathrm{mut}}$ cells showed nuclear deformations. In young HGPS cells (passage nr. 13), a majority of nuclei had an oval appearance (75\%) comparable to normal wild type fibroblasts, but a fraction that increased with passage number showed characteristic abnormalities such as blebs or herniations, invaginations and holes and especially, folds (Fig. 1).

To further characterize the differences in nuclear shape, we used a quantitative approach. A set of shape descriptors was extracted from DAPI counterstained

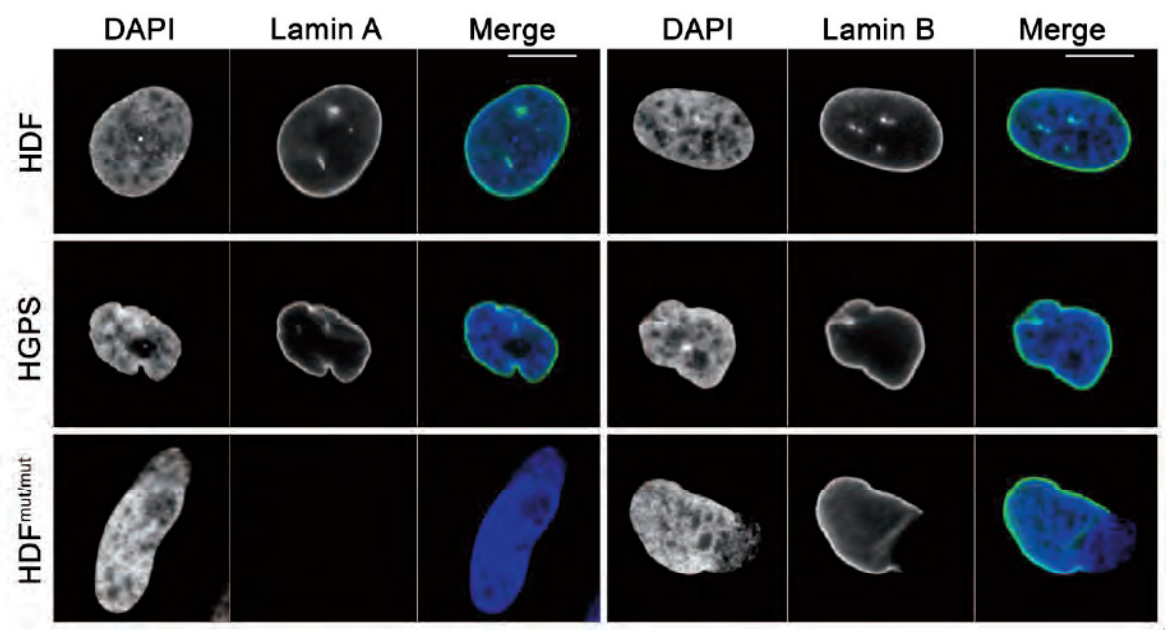

Figure 1: Confocal sections of representative nuclei of human fibroblasts from a healthy person (NHDF), a progeria patient (HGPS) and lamin $\mathrm{A} / \mathrm{C}$ deficient patient $\left(\mathrm{HDF}^{\mathrm{mut} m \mathrm{mut}}\right)$ after immunostaining for lamin $\mathrm{A}$ or lamin B (green) and counterstaining with DAPI (blue). Scale bars represent $10 \mu \mathrm{m}$. 

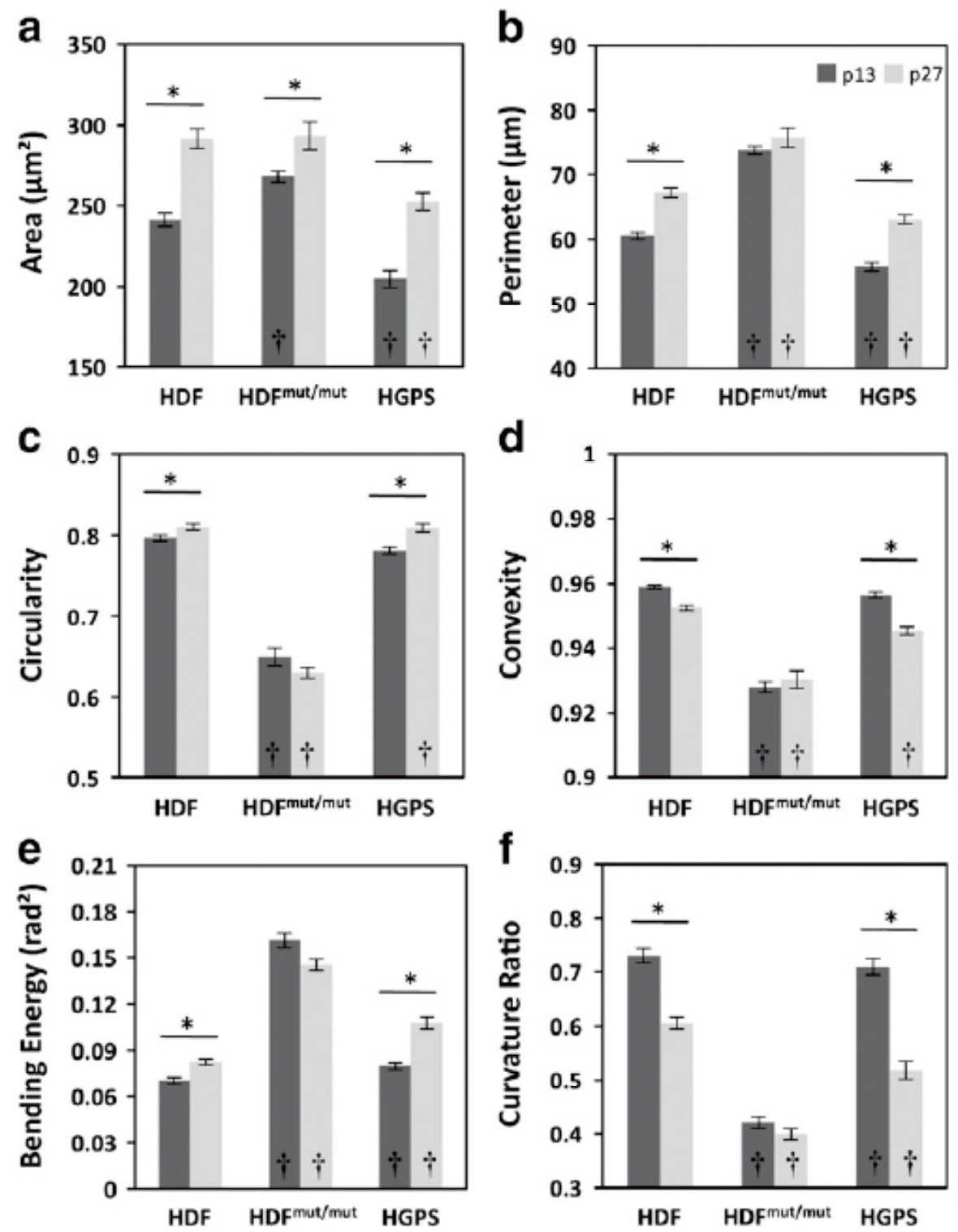

Figure 2. Comparison of the average shape descriptors area, perimeter, circularity, convexity, bending energy and curvature ratio of the three cell types NHDF, HDF ${ }^{\mathrm{mut} / \mathrm{mut}}$ and HGPS counterstained with DAPI. Bars represent average values ( \pm sem) passage number 13 (black) and passage number 27 (grey). Significant differences with respect to the passage matched control (NHDF) are marked by a dagger, indicated in the resp. bar. Within the cell type all parameters differed significantly between passage numbers for NHDF and HGPS (indicated by an asterisk), whereas in $\mathrm{HDF}^{\mathrm{mut} / \mathrm{mut}}$ this was only the case for nuclear area. ( $\mathrm{n}= \pm 300$ nuclei per cell type per passage number, data obtained from three independent experiments). 
cells for each cell type ( \pm 300 nuclei), using widefield mosaic microscopy and image analysis. The average values are represented in Fig. 2A general phenomenon, observed in all cell types, was a significant increase in cross-sectional area of the fibroblast nuclei with passage number (although for $\mathrm{HDF}^{\mathrm{mut} / \mathrm{mut}}$ to a lesser extent). In fact, all parameters significantly differed between passage number 13 and passage number 27 for NHDF and HGPS, indicating that these cells become more deformed with replicative age. No significant changes were observed between the two passage numbers of $\mathrm{HDF}^{\mathrm{mut} / \mathrm{mut}}$ cells. There were significant differences between cell types as well. In comparison with normal fibroblasts, early passage $\mathrm{HDF}^{\mathrm{mut} / \mathrm{mut}}$ cells were larger and showed a significantly lower circularity and convexity. All shape differences, except nuclear size, remained significant between these cells at the higher passage number. In our hands, HGPS cells of passage number 13 were smaller but did not show a dramatic reduction in the aforementioned shape descriptors. The difference only became apparent in cells of a later passage number (passage nr. 27).

The bending energy, which is more sensitive to local edge variations, did reveal the presence of stronger curvature in HGPS cells, in both early and late passage cells. Complementation of HDF $^{\text {mut/mut }}$ cells by transient transfection of pEGFP-LaminA did not result in normalization of nuclear shape. After transfection, accumulation of EGFPcoupled lamin A was found at local regions of constriction within the nucleus or in large-meshed networks (Fig. 3A,B). The local depletion of lamin B was not recovered after transfection either (Fig. 3C). 

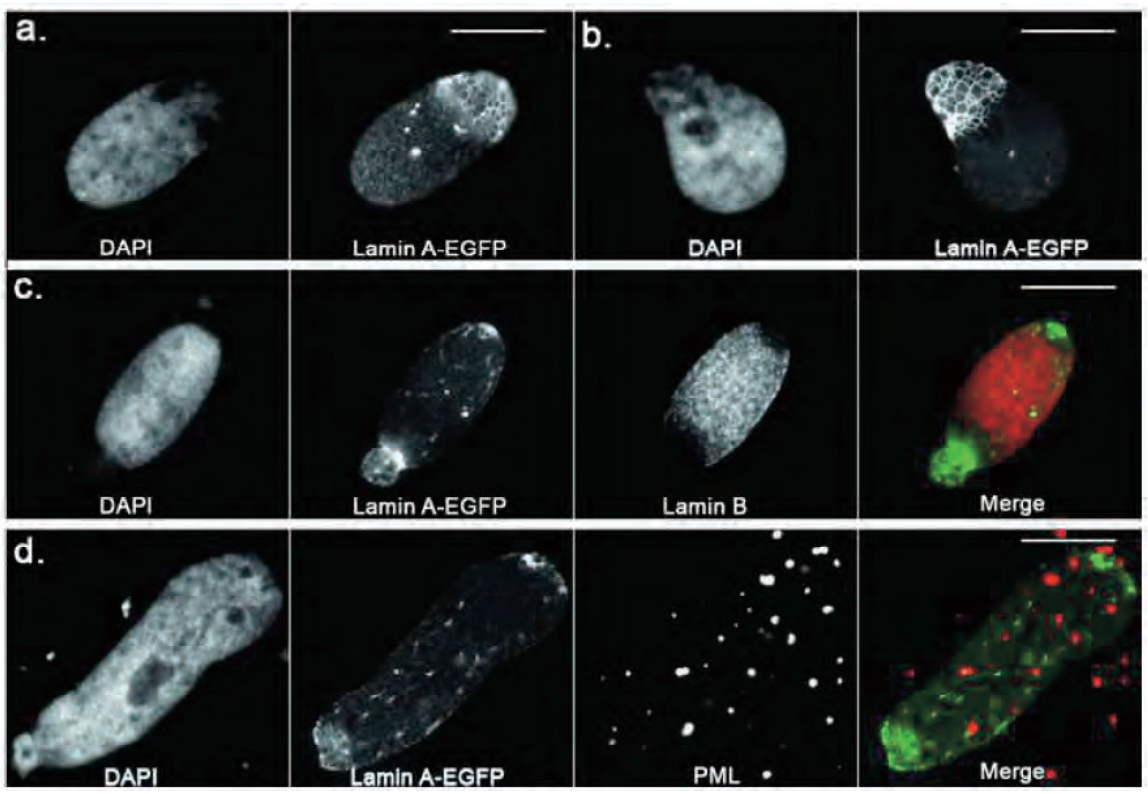

Figure 3. Complementation of $\mathrm{HDF}^{\text {mut/mut }}$ cells by transfection with LMNA-EGFP is not sufficient to restore nuclear phenotype. a,b Widefield recordings showing two $\mathrm{HDF}^{\mathrm{mut} / \mathrm{mut}}$ nuclei expressing lamin-EGFP $24 \mathrm{~h}$ after transfection. c. Montage of a cell transfected with LMNA-EGFP (green) after immunostaining for lamin B (red), demonstrating that lamin B is still heterogeneous in localization. d. Montage of a LMNAEGFP (green) transfected $\mathrm{HDF}^{\mathrm{mut} / \mathrm{mut}}$ cell after immunostaining for PML-protein (red), which still resides throughout the cell. Scale bars represent $10 \mu \mathrm{m}$.

\section{Nuclear mobility and deformation is dramatically increased in $\mathrm{HDF}^{\mathrm{mut} / \mathrm{mut}}$}

To study the nuclear dynamics, cells were transfected with a vector expressing the telomere-binding fusion protein mCitrine-hTRF2. In early experiments we found that overexposure to light can cause cells to move and deform abnormally. In order to image cells under minimally (photo-) toxic conditions, which is essential to this type of investigation, we made use of controlled light exposure microscopy (CLEM), a confocal technique that reduces illumination to the objects of interest. For each cell type \pm 10 nuclei with moderate and correct expression of the fusion protein were imaged for $80 \mathrm{~min}$ at $2 \mathrm{~min}$ intervals. Cells correctly expressing mCitrine-hTRF2 typically showed a punctuate pattern of telomere-localized protein within a nuclear pool of non-bound protein. The background nuclear signal was used for determining 
the nuclear outlines. This approach is particularly useful for documenting nuclear plasticity in $\mathrm{HDF}^{\mathrm{mut} / \mathrm{mut}}$ cells. Chromatin in these cells is dramatically reorganized, especially in extrusions, with large peripheral euchromatic domains, domains with a frayed appearance and nuclear regions that appeared devoid of chromatin. Upon transfection, free mCitrine-hTRF2 fills up the nucleus, thereby revealing the complete boundary. The nuclear outlines allowed for analyzing shape deformation, while the telomeric signals were used for analyzing telomere dynamics, as schematically represented in Fig. 4A (see also movie 1-3, Supplementary Material). To obtain a general impression of the global nuclear dynamics, a correlation analysis was performed on the sum projections of the CLEM recordings (Fig. 4B). To avoid bias from superimposed cellular or nuclear mobility (i.e. displacement and/or rotations) the images were rigidly registered before the analysis. Then, the average Pearson correlation coefficient was determined between consecutive time points, to determine short-term variability. HGPS and HDF cells maintained a higher correlation and showed less fluctuation in correlation coefficient across the time range than $\mathrm{HDF}^{\mathrm{mut} / \mathrm{mut}}$ cells. Likewise, the variation between nuclei (represented by the standard deviation per time point) was significantly higher in $\mathrm{HDF}^{\mathrm{mut} / \mathrm{mut}}$ cells. Notably, correlation and cellto-cell variability profiles of HGPS cells demonstrated fewer fluctuations than NHDF. The temporal autocorrelation curves were determined as well to quantify long-term variability. The autocorrelation curve of $\mathrm{HDF}^{\mathrm{mut} / \mathrm{mut}}$ cells showed a steep decrease with increasing time lag; whereas the other two cell types showed little difference and both maintained a high autocorrelation throughout the recording period.

Decrease in correlation is a combined effect of nuclear deformation and internal motion. We have addressed these two phenomena separately. We first quantified nuclear deformation in terms of shape descriptors, using the nuclear outlines from the sum projections (Fig. 5). Individual profiles of $\mathrm{HDF}^{\mathrm{mut} / \mathrm{mut}}$ nuclei show extensive fluctuations through time. Although both HGPS and NHDF show rigid shape descriptor profiles, individual NHDF nuclei appeared to show slightly more variation than HGPS nuclei. However, when we statistically compared the average coefficient of variation (short-term variability) and the range (long-term variability) for these metrics we failed to demonstrate a significant difference (Table 1). In contrast, differences between NHDF and $\mathrm{HDF}^{\mathrm{mut} / \mathrm{mut}}$ were significant. 

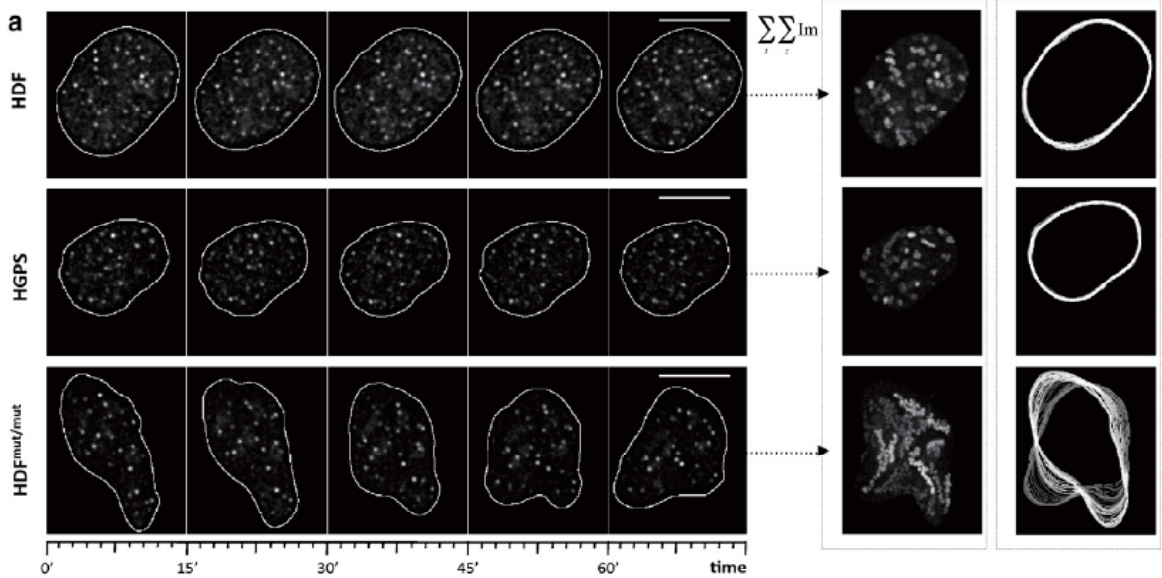

b

Time-Point Correlation Fluctuations
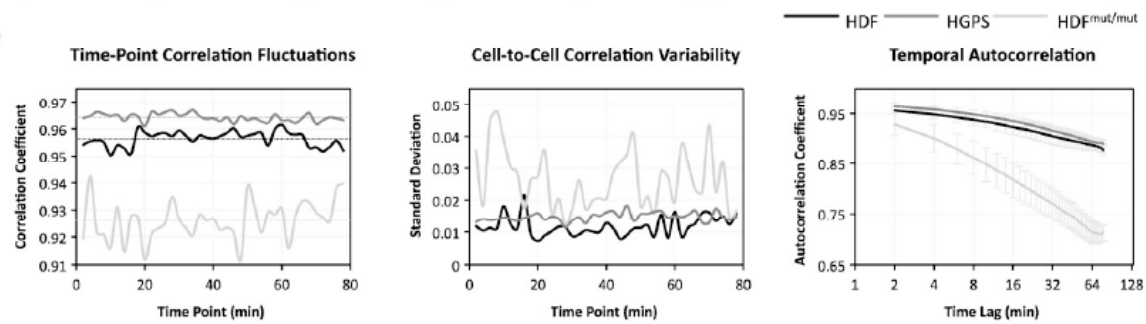

Figure 4. (a) Schematic representation of live cell analysis. The three types of human fibroblasts were transiently transfected with pmCitrine-hTRF2 and recorded with CLEM every 2 min for at least 80 min. Representative deconvolved images at selected time points (0, 20, 40, 60 and $80 \mathrm{~min})$, show a typical punctuate telomere-localized pattern within a pool of nuclear background protein. The latter was used for automatically demarcating the nuclear boundary (white outlines). Nuclei were registered as described in the Materials and methods section. The sum projections of the confocal 4D images across time demonstrate intranuclear mobility while the time-coded grey-tone sum projections of the nuclear outlines illustrate nuclear shape deformation. Scale bars represent $10 \mu \mathrm{m}$. (b) Correlation analysis on registered CLEM recordings of nuclei from the three cell types, transiently expressing mCitrine-hTRF2. At least 8 nuclei were analyzed per cell type. The left graph represents the average Pearson correlation coefficient between consecutive time points per cell type; the middle graph displays the standard deviation on these values, to demonstrate the cell-to-cell variability within a given cell type and the right graph depicts the average autocorrelation curves $( \pm$ std) as a function of time lag. 

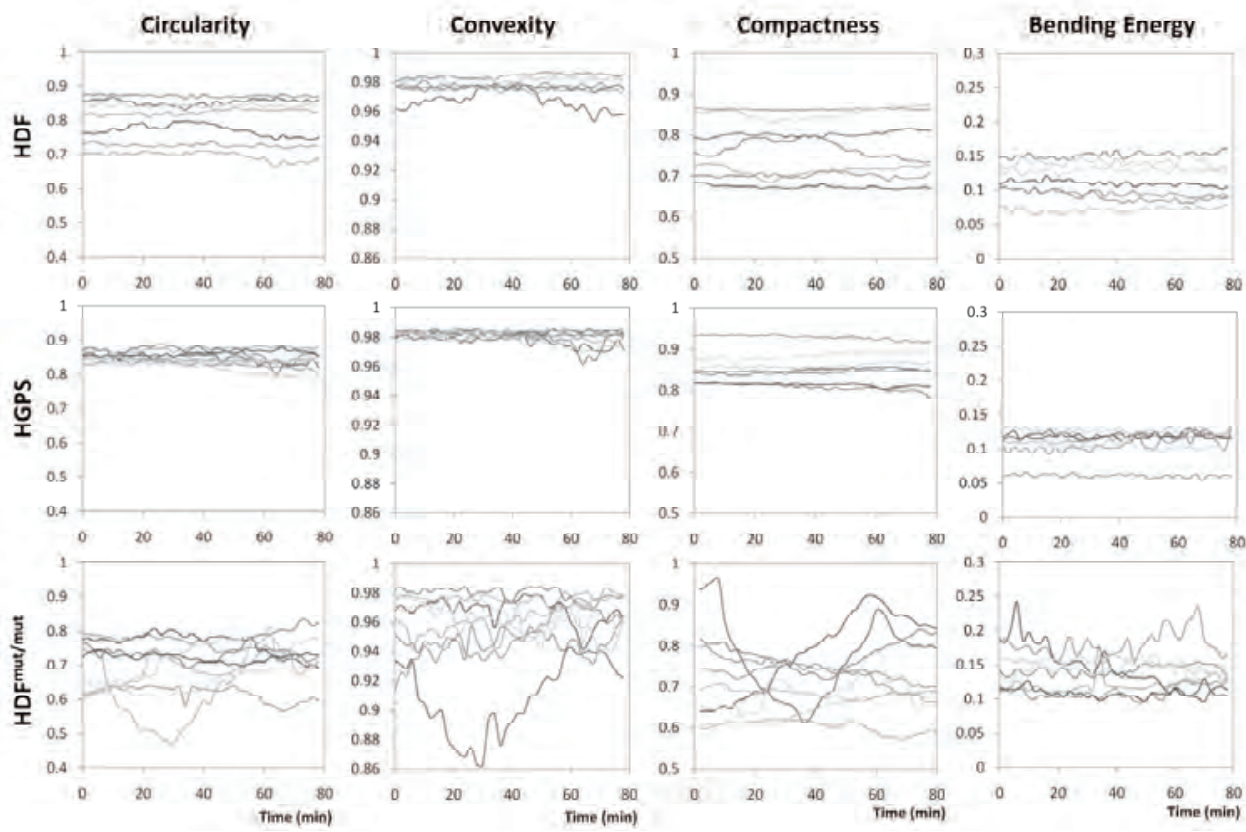

Figure 5. Shape deformation in the three cell types over time, represented by the shape descriptors circularity, convexity, compactness and bending energy. Each line represents the value fluctuations of an individual nucleus (data obtained from two independent experiments).

Table 1. Quantification of shape analysis on live recordings: average range between maximal and minimal value \pm std and average coefficient of variation $(\mathrm{CoV}) \pm$ std.

* Sign. different from NHDF.

\begin{tabular}{|c|c|c|c|c|c|}
\hline & & Circularity & Convexity & Compactness & $\begin{array}{l}\text { Bending } \\
\text { Energy }\end{array}$ \\
\hline \multirow{3}{*}{$\begin{array}{l}\text { Average } \\
\text { Range }\end{array}$} & NHDF & $0.03 \pm 0.02$ & $0.009 \pm 0.006$ & $0.03 \pm 0.02$ & $0.02 \pm 0.01$ \\
\hline & HGPS & $0.03 \pm 0.01$ & $0.007 \pm 0.003$ & $0.03 \pm 0.01$ & $0.02 \pm 0.01$ \\
\hline & HDF $^{\text {mut/mut }}$ & $0.14 \pm 0.08 *$ & $0.041 \pm 0.019^{*}$ & $0.14 \pm 0.10^{*}$ & $0.09 \pm 0.10 *$ \\
\hline \multirow{3}{*}{$\begin{array}{l}\text { Average } \\
\text { CoV (\%) }\end{array}$} & NHDF & $1.07 \pm 0.66$ & $0.23 \pm 0.17$ & $1.03 \pm 0.79$ & $4.97 \pm 1.52$ \\
\hline & HGPS & $0.96 \pm 0.53$ & $0.18 \pm 0.11$ & $0.94 \pm 0.56$ & $4.67 \pm 2.34$ \\
\hline & HDF $^{\text {mut/mut }}$ & $5.85 \pm 4.01 *$ & $1.17 \pm 0.69 *$ & $5.53 \pm 3.64 *$ & $10.86 \pm 7.30 *$ \\
\hline
\end{tabular}




\section{Distinct subnuclear mobility regimes revealed by telomere tracking}

In agreement with previous observations in cancer cells [43], telomeres were distributed throughout the entire volume of the nucleus. To determine differences in dynamic behaviour we investigated subnuclear mobility by tracking telomere spots in living fibroblasts. First, we quantified long-term mobility in CLEM recordings (Fig. 6). The peak displacement (Fig. 6a), which represents the maximum distance with respect to the origin (the first time point), as well as the cumulative distance (Fig. 6b), travelled throughout the time period, was significantly different between all three cell types: telomeres in $\mathrm{HDF}^{\mathrm{mut} / \mathrm{mut}}$ cells demonstrated an overall increased mobility whereas HGPS cells show reduced telomere dynamics with respect to normal fibroblasts. The maximum cumulative lateral displacement observed within a time window of $80 \mathrm{~min}$ was $7 \mu \mathrm{m}, 9 \mu \mathrm{m}, 11.5 \mu \mathrm{m}$ for HGPS, NHDF and HDF ${ }^{\mathrm{mut} / \mathrm{mut}}$, respectively.

The maximal peak displacement in 3D for these three cell types was $2.6 \mu \mathrm{m}, 3.1 \mu \mathrm{m}$ and $6.9 \mu \mathrm{m}$, respectively. An example of an extremely large individual displacement, independent of the global telomeric constellation, in $\mathrm{HDF}^{\mathrm{mut} / \mathrm{mut}}$ cells is shown in Fig. 7. A useful parameter in mobility analysis is the mean square displacement (MSD). The MSD plot describes a relationship between the square distance travelled by a particle and increasing time intervals. This relationship is directly correlated with diffusion parameters, according to the general formula: $M S D=6 \Gamma \Delta \alpha$. For free diffusion, the MSD plot is a straight line, the anomalous diffusion coefficient $(\alpha)$ equals 1 and $\Gamma=\mathrm{D}$, the diffusion coefficient. Deviation from linearity, provides a measure for spatial obstruction $(\alpha<1)$ or directionality of movement $(\alpha>1)$. Although telomeres and chromatin loci in general show limited motion [54, 55], different regimes of motion were present. To capture all types of motion, we applied the general formula of anomalous diffusion. For each telomere track we calculated the MSDs and from the best weighted fit we deduced the parameters $\alpha$ and $\Gamma$. The overall telomere mobility was constrained $(\alpha<1)$, which can also be deduced from the slope of the log$\log$ plot (Fig. 6d). The median $\alpha$ did not differ significantly between NHDF $(0.31$, SEM=0.01) and HGPS (0.29, SEM=0.01), but $\mathrm{HDF}^{\mathrm{mut} / \mathrm{mut}}$ cells had a significantly higher $\alpha$ of 0.58 (SEM=0.02). The interpretation of the transportation coefficient is not straightforward, since it is related to the diffusion coefficient in a non-linear manner $(\mathrm{D}=\Gamma \Delta \alpha-1)$. To describe the mobility of intranuclear particles in terms of diffusion coefficients, we made use of the moving corral model, in which a nuclear particle experiences confined diffusion (Dfast) in a corral of radius rc, that can also translocate by slower diffusion (Dslow) [56]. 
This framework was first used to model mobility of nuclear bodies, but has recently also been used to describe telomere mobility [52]. Moving corral fits were determined for individual and average MSDplots of each cell type (Fig. 6e). Differences in corral radius revealed that global confinement increases in the order $\mathrm{HGPS}<\mathrm{NHDF}<\mathrm{HDF}^{\mathrm{mut} / \mathrm{mut}}$. Similar trends were observed for both diffusion coefficients (Table 2). The high absolute value for the slow diffusion coefficient of $\mathrm{HDF}^{\mathrm{mut} / \mathrm{mut}}$ is consistent with the high amount of telomere tracks we found with directed motion $\left(\alpha>1 ; 11 \%\right.$ in $\mathrm{HDF}^{\mathrm{mut} / \mathrm{mut}}$ vs. $1 \%$ in NHDF) and is likely to reflect the mobility of large chromatin domains. Interestingly, the fast diffusion coefficient, which reflects smaller time scale motion, also appeared to be higher. Therefore, we investigated short-term mobility in more detail, by means of highly time-resolved recordings (220 ms intervals). To remain scarce with light, we acquired brief $(<1 \mathrm{~min})$ widefield recordings from a single focus plane with a sensitive EM-CCD camera and attenuated the excitation light with a neutral density filter. The average MSD plots followed a similar trend as the long-term movements (Fig. 5f) and the corral fitting analysis also revealed a similar order of mobility between the cell types (Table 2). 

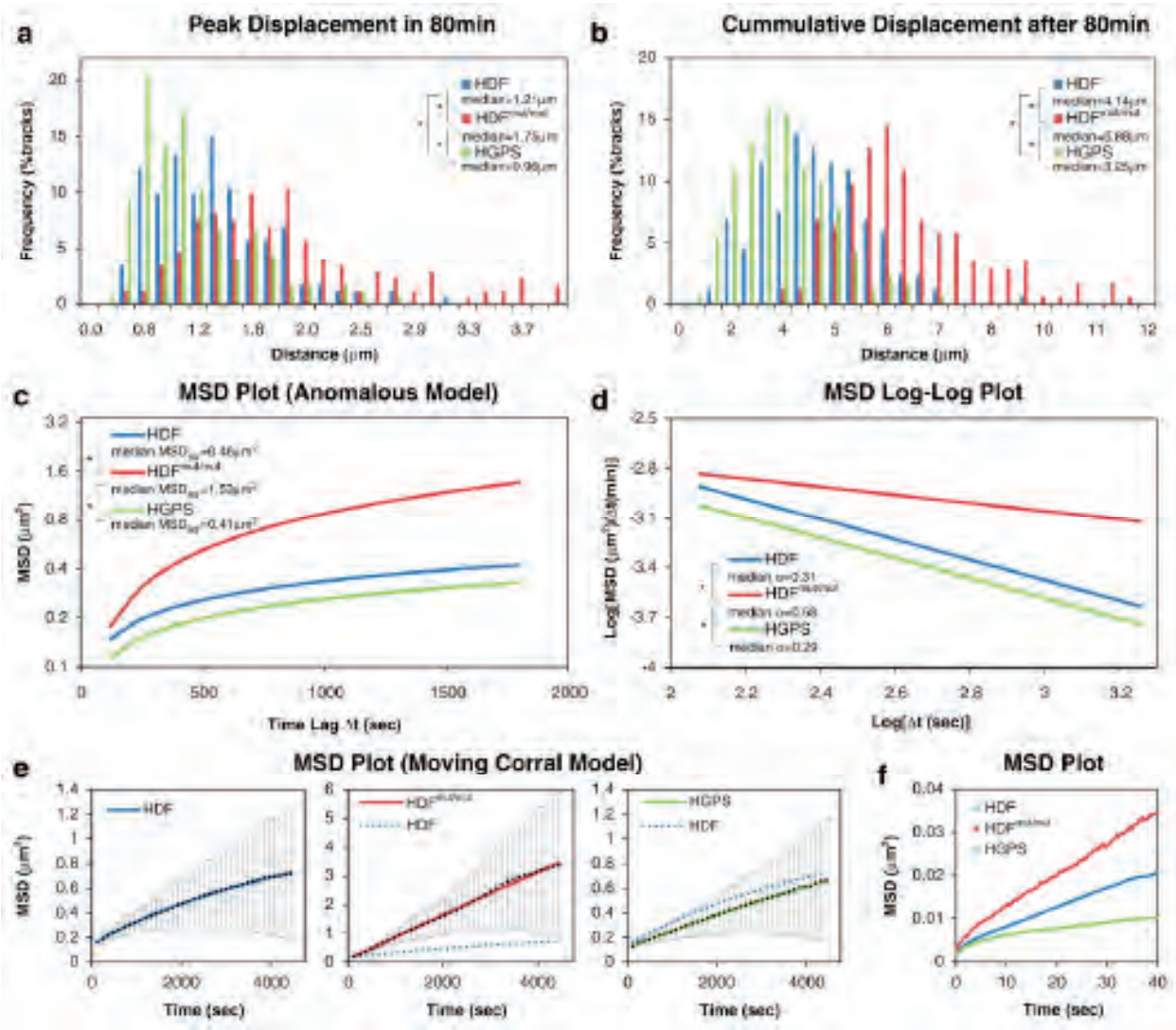

Fig. 6. Quantitative analysis of long-term telomere (a-e) and short-term (f) mobility in fibroblast nuclei of the three cell types. Long-term mobility was determined on CLEM recordings. (a) Histogram of telomere peak displacement (maximal distance to origin) over a time range of $80 \mathrm{~min}$. (b) Lateral cumulative (total) displacement after $80 \mathrm{~min}$. (c) Best fit to the average MSD plot, pooled per cell type, as a function of time lag $\Delta \mathrm{t}$, determined according to the anomalous diffusion model. (d) Plot of $\log (\mathrm{MSD} / \Delta \mathrm{t})$ versus $\log (\Delta \mathrm{t})$ shows to demonstrate the subdiffusive character of telomere movements $(\alpha<1)$. Because of the non-normal distribution of tracking parameters, the median values of relevant parameters are shown: peak displacement (a), cumulative displacement (b), MSD after $80 \mathrm{~min}$ (c) and anomalous diffusion coefficient $\alpha$ (d). Statistically significant differences $(\mathrm{p}<0.05)$ between cell types are marked by an asterisk. NHDF $(n=173)$, $\operatorname{HDF}^{\text {mutmut }}(\mathrm{n}=173)$, HGPS ( $\left.\mathrm{n}=184\right)$. (e) The pooled MSD values were also fitted according to the moving corral model. Black dots represent the average values, error bars the standard deviations and the coloured line the best fit. Note the scaling difference of the Y-axis between HDF $^{\text {mut } / m u t}$ and NHDF and HGPS. (f) MSD plot of pooled telomere tracks of short-term time-resolved widefield recordings for the three cell types. Average values are shown NHDF $(n=466)$, HGPS $(n=370)$ and $\operatorname{HDF}^{\text {mutmut }}(n=460)$. Data obtained from three independent experiments. 

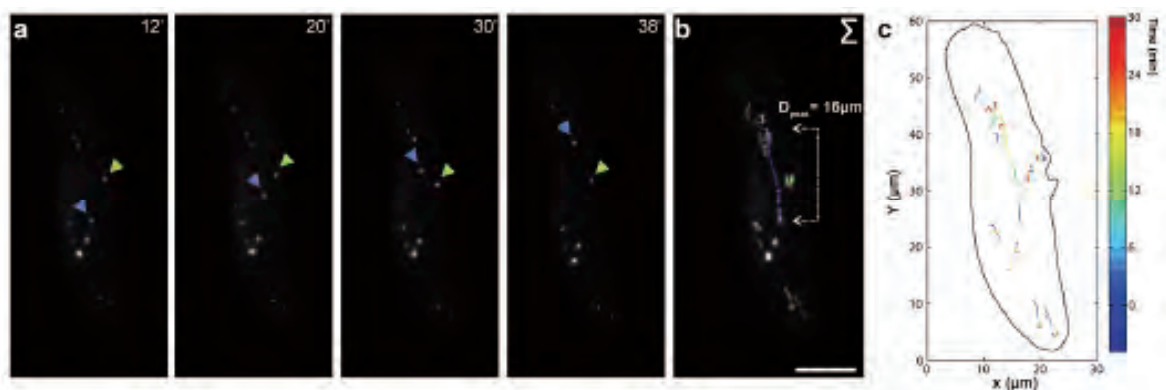

Fig. 7. Individual telomere mobility in $\mathrm{HDF}^{\mathrm{mut} / \mathrm{mut}}$ cells can be high. (a) A single telomere (blue arrowhead) undergoes directional displacement by almost $16 \mu \mathrm{m}$, with respect to the other telomeres. For clarity, only one other telomere is indicated (green arrowhead). (b) Maximum projection of the time stack. Scale bar represents $10 \mu \mathrm{m}$. (c) Colour coded time projection of the tracks of all the telomeres that could be tracked throughout the time recording, plotted in the nuclear outline.

Table 2. Mobility metrics from moving corral model fit on long term CLEM recordings (80min, sampled at $2 \mathrm{~min}$ interval) and short term widefield (WF) EM-CCD recordings (44sec, sampled at $0.22 \mathrm{sec}$ intervals). Legend: $r_{c}=$ corral radius, $D_{s}=$ slow diffusion coefficient and $D_{f}=$ fast diffusion coefficient.

\begin{tabular}{|c|c|c|c|c|c|}
\hline & Cell type & \# tracks (nuclei) & $\mathbf{r}_{\mathrm{c}}(\boldsymbol{\mu m})$ & $\mathbf{D}_{\mathrm{s}}\left(\mu \mathbf{m}^{2} / \mathbf{s}\right)$ & $D_{\mathrm{f}}\left(\mu \mathbf{m}^{2} / \mathbf{s}\right)$ \\
\hline \multirow{3}{*}{ CLEM } & NHDF & $173(8)$ & 0.64 & $1.0 \mathrm{E}-05$ & $3.5 \mathrm{E}-05$ \\
\hline & HGPS & $184(8)$ & $0.50^{\mathrm{a}}$ & $1.2 \mathrm{E}-05^{\mathrm{a}}$ & $2.2 \mathrm{E}-05^{\mathrm{a}}$ \\
\hline & HDF $^{\text {mut/mut }}$ & $173(9)$ & $1.83^{\mathrm{a}}$ & $7.3 \mathrm{E}-05^{\mathrm{a}}$ & $13 \mathrm{E}-05^{\mathrm{a}}$ \\
\hline \multirow{7}{*}{ WF } & NHDF & $466(31)$ & 0.045 & $9.5 \mathrm{E}-05$ & 4.6E-04 \\
\hline & HGPS & $370(29)$ & $0.039^{b}$ & $3.7 \mathrm{E}-05^{\mathrm{b}}$ & $4.2 \mathrm{E}-04^{\mathrm{b}}$ \\
\hline & $\mathrm{HDF}^{\mathrm{mut} / \mathrm{mut}}$ & $460(39)$ & $0.053^{b}$ & $16 \mathrm{E}-05^{\mathrm{b}}$ & $5.4 \mathrm{E}-04^{\mathrm{b}}$ \\
\hline & NHDF + shLuc & $195(23)$ & 0.042 & $8.8 \mathrm{E}-05$ & $5.7 \mathrm{E}-04$ \\
\hline & $\begin{array}{l}\text { NHDF } \\
\operatorname{shLamin} A / C\end{array}$ & $296(27)$ & $0.054^{\mathrm{c}}$ & $10 \mathrm{E}-05$ & $5.4 \mathrm{E}-04$ \\
\hline & HGPS + DMSO & $203(22)$ & 0.035 & $4.1 \mathrm{E}-05$ & $4.3 \mathrm{E}-04$ \\
\hline & HGPS + FTI & $188(20)$ & $0.046^{\mathrm{d}}$ & $12 \mathrm{E}-05^{\mathrm{d}}$ & $5.0 \mathrm{E}-04$ \\
\hline
\end{tabular}




\section{Experimental manipulation alters telomere mobility regimes}

To investigate whether the role of incorrect lamin processing was the causative factor for decreased telomere mobility in HGPS cells, we treated cells for $72 \mathrm{~h}$ with the farnesyl inhibitor FTI-276. Analysis of short-term recordings revealed a significant increase (two-fold in terms of MSD) in telomere mobility in HGPS after incubation with FTI (Fig. 8a). All fitting parameters were higher, of which corral radius and slow diffusion coefficient significantly, in FTI-treated versus mock treated cells (Table 2).
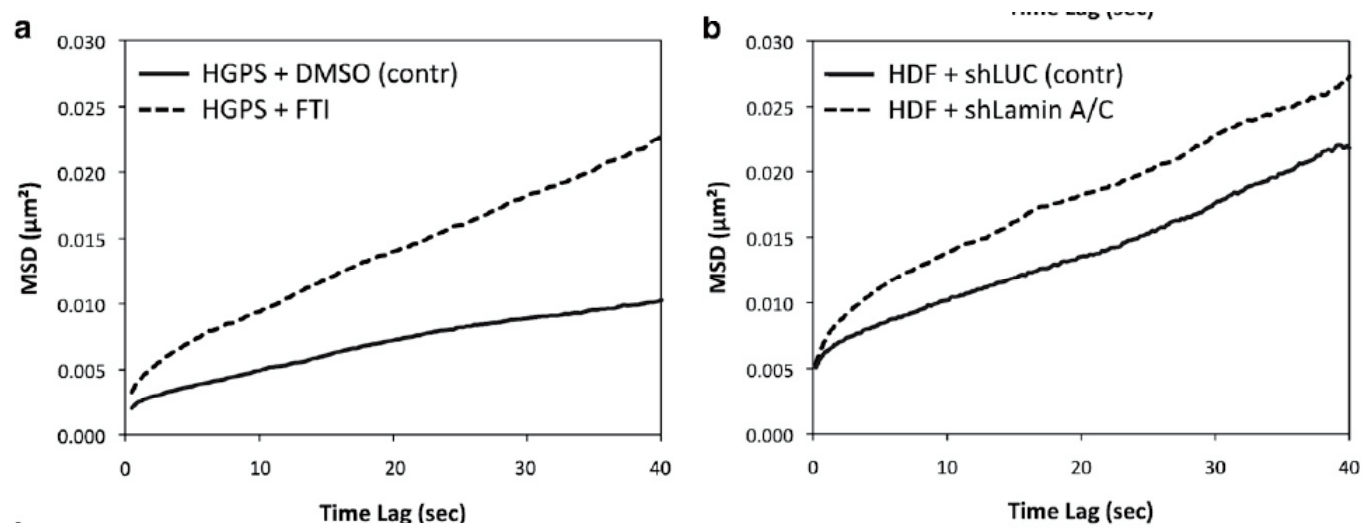

Fig. 8. Average MSD plots of telomere mobility after experimental manipulation. (a) Fibroblasts from a HGPS patient were treated with $1 \mu \mathrm{M}$ FTI for $72 \mathrm{~h}$ and transfected with pmCitrine-hTRF2 after which a recording of 200 time points at $220 \mathrm{~ms}$ intervals was acquired and telomere mobility was determined. Control cells were treated with equal amounts of DMSO. (b) Wild type fibroblasts (NHDF) were transfected with pmCitrine-hTRF2 and cotransfected with psiRNA42-LaminA/C or psiRNA-Luc (control) and imaged after $48 \mathrm{~h}$.

To determine whether the increase in telomere mobility in $\mathrm{HDF}^{\mathrm{mut} / \mathrm{mut}}$ was due to the absence of functional lamin, we assessed the effect of silencing lamin $\mathrm{A} / \mathrm{C}$ in normal cells. To achieve this, wild type NHDF were transfected with pmCitrine-hTRF2 and a vector expressing a short hairpin sequence targeting LMNA transcripts (psiRNA42LaminA/C). Control cells were cotransfected with a short hairpin expressing vector targeting LUC instead. Both shRNA vectors contained a fluorescent marker (tagRFP) for identification of transfected cells. Immunofluorescence staining for lamin A after knockdown showed up to $90 \%$ reduction of lamin A expression in $50 \%$ of all cells (data not shown). $48 \mathrm{~h}$ after transfection short-term recordings were acquired of double positive cells. A small but significant increase ( $20 \%$ in terms of MSD) in telomere mobility was observed in cells transfected with psiRNA42-LaminA/C with respect 
to control cells (Fig. 8b). All corral fitting parameters were elevated after knockdown, but only the corral radius was significantly different from the control (Table 2). 


\section{Discussion}

\section{Lamin A/C shapes the nucleus}

It has been established that lamins play an important role in nuclear mechanics. Especially lamin $\mathrm{A} / \mathrm{C}$ seems to be involved in maintaining nuclear integrity [57]. Indeed, from our observations concerning nuclear shape and deformation we can infer that lamin $\mathrm{A} / \mathrm{C}$ tunes the rigidity of the lamina and determines shape. We have presented a quantitative description of nuclear shape which allowed objective and statistically sound comparison between different cells. We found nuclear shape to be dramatically affected in cells from a patient with a nonsense mutation in the LMNA gene, regardless of the passage number. Transfection of cells with pEGFP-LaminA did not improve nuclear shape. Instead, it resulted in accumulation of EGFP-lamin A at the regions previously devoid of any lamin or at the transition zones. One reason might be that the full lamin spectrum, including lamin $\mathrm{C}$, is required for structural recovery of the lamina. However, it was shown that complementation with prelamin $\mathrm{A}$ in LMNA-/- MEFs does not restore nuclear shape abnormalities [57]. Another possibility is that the recovery of the lamina requires a complete cell cycle and that the lamina and nuclear shape might be restored only after mitosis. Arguments for this can be found in the requirement of a cell division for correct repositioning of lamin targeted genes $[17,58]$ and in the slow turnover rates of lamin A in the lamina [6]. Unfortunately, we have not been able to maintain cells in culture long enough after transfection to confirm this hypothesis.

In HGPS cells, the nucleus became progressively more deformed, with increasing passage number. Although early passage HGPS cells did not differ markedly from normal cells, well-chosen parameters such as local curvature descriptors, revealed subtle differences in a subfraction of the cells. Progressive changes in the HGPSnucleus have been documented before and are considered to be due to accumulation of progerin at the nuclear envelope [16]. Both HGPS and $\mathrm{HDF}^{\mathrm{mut} / \mathrm{mut}}$ cells show nuclear abnormalities, indicating the importance of correct lamin $\mathrm{A} / \mathrm{C}$ expression. On the other hand, the two cell types show very distinct dynamics with respect to normal fibroblasts and with respect to each other: whereas complete deficiency in lamin $\mathrm{A} / \mathrm{C}$ leads to a dramatic plasticity of the nucleus, HGPS cells maintain a rigid shape throughout time. This correlates well with recent findings on decreased nuclear shape stability in lamin $\mathrm{A} / \mathrm{C}$ deficient mouse fibroblasts [57] and increased nuclear stiffness in late passage HGPS cells [23], respectively. HGPS nuclei showed fewer fluctuations in shape 
throughout time compared with normal fibroblasts, although the difference was not significant. In this experiment, we have only considered a middle-passage. The difference may become more pronounced with later passages as progerin accumulates further. Using ballistic intracellular microrheology it was elegantly shown that lamin $\mathrm{A} / \mathrm{C}$ deficiency results in a decreased cytoplasmic stiffness and elasticity [59]. This cytoplasmic softening is considered to be due to disrupted nucleo-cytoskeletal interactions. Indeed, in laminopathic mouse model cells, components of the LINC complex are mislocalized [26] and disruption of the LINC complex affects cellular mechanical stiffness to a degree that equals that of lamin A deficient mouse cells [27]. Disrupted nucleo-cytoskeletal interactions and cytoplasmic softening could in turn also account for altered nuclear shape and plasticity. A possible mechanism for shape regulation may reside in a recently discovered perinuclear actin cap [60]. Abolishment of this cap by treatment with latrunculin B resulted in loss of nuclear shape control. Similarly, laminopathic mouse cells or mouse cells with disrupted LINC complexes are more frequently found without an actin cap or with a disrupted cap and have a reduced ability to regulate nuclear shape. Since $\mathrm{HDF}^{\mathrm{mut} / \mathrm{mut}}$ cells completely lack functional lamin A and show disrupted nucleo-cytoskeletal interactions [22], they may also have a disrupted actin cap causing the loss of shape control. The general importance of preserved nucleo-cytoskeletal connections is further underlined by the observations of spinning nuclei in mouse cells lacking lamin B1, which could be immobilized by reexpressing lamin B1 or over expressing larger nesprin isoforms [25].

Thus, loss of nucleo-cytoskeletal connections could account for the increased nuclear plasticity and dynamics found in $\mathrm{HDF}^{\mathrm{mut} / \mathrm{mut}}$. Why this plasticity was not detected in HGPS cells, might be explained by the presence of sufficient levels of normal lamin A. In contrast to mouse model cells for progeria which have a homozygous mutation in the LMNA gene (LMNAL530P/L530P), human progeria is caused by a single dominant point mutation (LMNA+/G608G). The (slightly) increased rigidity found in HGPS cells may be due to the accumulation of progerin. Since, farnesyl groups increase lipophilicity and promote membrane interactions, progerin (which remains farnesylated) is likely to increase rigidity of the nucleus [58]. Progerin could also increase nuclear stiffness by its altered polymerization and subsequent reduced mobility in the nuclear lamina [19].

\section{Nuclear dynamics and telomere mobility in human fibroblasts}

The nuclear lamina is thought to provide a framework for organizing nuclear envelope 
structure and anchoring interphase chromatin at the nuclear periphery [61]. Furthermore, lamins are distributed throughout the nucleoplasm, where they appear to be essential for DNA replication and RNA polymerase II transcription [62]. Complete deficiency of lamin $\mathrm{A} / \mathrm{C}$ leads to a dramatic reorganization of the nucleus, with, next to an altered nuclear shape, an eroded periphery and reorganized chromatin distribution as a consequence. HGPS fibroblasts also display changes in nuclear organization, most notably a loss of peripheral heterochromatin [30]. This sparked our curiosity on how mutations in lamins affect chromatin mobility. Chromatin movement has generally been found to exhibit characteristics of an obstructed random walk, meaning that a chromosomal locus randomly samples a region with a defined radius of constraint [63, 64]. The source of this constraint is unknown, but it could arise from intrinsic viscosity of the nucleoplasm [65], from association with other chromatin fibers, and/or from interactions with less mobile proteins or structural elements such as lamins. Using live cell imaging, we followed individual telomere domains and quantitatively characterized the constraints imposed upon their mobility. Recently, a confined diffusion model was proposed for telomere mobility on different time scales in U2OS cells [52]. In this study, we could confirm the presence of different mobility regimes with confinement regions of about $50 \mathrm{~nm}$ radius at the seconds time scale and about $500 \mathrm{~nm}$ radius at the hour time scale. The diffusion coefficients we calculated were slightly lower than documented before, but of comparable magnitude $(1 \mathrm{E}-04 \mu \mathrm{m} 2 / \mathrm{s}-$ $1 \mathrm{E}-05 \mu \mathrm{m} 2 / \mathrm{s})$. This might be attributed to the differences in time intervals but also in analysis: we used the distance change of individual particles instead of inter-individual distance changes between particles. Furthermore, we assessed a different cell type and it has been shown that diffusion coefficients of subchromosomal (replication) foci in nuclei and within chromosome territories can differ between cell types [50].

\section{Increased telomere mobility in $\mathrm{HDF}^{\mathrm{mut} / \mathrm{mut}}$ cells}

A global telomere analysis on different time scales revealed that telomere mobility is higher and less confined in $\mathrm{HDF}^{\mathrm{mut} / \mathrm{mut}}$ cells compared with normal fibroblasts. A major contribution stems from the slowest component, which reflects the movement of large chromatin domains within the nucleus and is correlated with the extensive morphological changes experienced by these cells. This implies that in the absence of a restraining element such as a fully functional lamina chromatin can undergo large displacements with respect to other regions over time. Intriguingly, also on smaller time scales telomere mobility was increased, indicating more local freedom of movement. To verify this we acquired highly-resolved recordings within a very short time window. Within this subminute time span morphological changes of the nucleus 
were negligible assuring local mobility analysis and excluding superimposed deformation-driven mobility. This approach confirmed the difference on the second time scale. We conclude that the overall nuclear mobility is increased in $\mathrm{HDF}^{\mathrm{mut} / \mathrm{mut}}$ cells. Thus, the presence of lamin $\mathrm{A} / \mathrm{C}$ appears essential in providing internal inertia. This could be achieved either through direct complexation - interactions between telomeres and lamins $[35,38,66]$ or other nuclear envelope proteins $[41,42]$ have been detected - via interactions with other chromatin domains, containing MARs [36], or through maintenance of the proper internal pressure. Nanoparticle tracking studies have revealed that the stiffness of the nucleus is about two-fold higher than that of the cytoplasm and that the high nuclear mesoscale viscosity contributes greatly in setting the pace of diffusion and directed transport of large macromolecular complexes [65]. Lamin A may influence this viscosity by controlling nuclear membrane shape and thus, nuclear size [67] but also by delineating nuclear microenvironments. Given the fact that most telomeres avoid the nuclear periphery and are distributed throughout the nuclear interior [41, this study], it is tempting to speculate on a more specific role for intranuclear lamins in tethering telomeres and thereby regulating telomere organization and mobility. Recently, a shift in telomere localization was observed towards the nuclear periphery in the absence of A-type lamins, indeed suggesting an active role of A-type lamins in the positioning of telomeres in the nuclear interior [68]. Moreover, Atype lamins were found to regulate telomere length, and structure, 53BP1 levels and overall genome stability. Since 53BP1 restricts the mobility of dysfunctional telomeres within the nucleus [69] it was suggested that A-type lamins provide a platform for the association of 53BP1 with dysfunctional telomeres, and regulate the mobility of dysfunctional telomeres for further processing by non-homologous end-joining [68].

\section{Increased telomere mobility in LMNA silenced NHDF cells}

In order to exclude the possibility that differences in telomere mobility between cell lines examined resulted from variations between cell lines, age and/or genetic background of the patient, we mimicked the effect of lamin A deficiency by reducing lamin A/C expression using RNA interference technology in NHDF cells. A small (in terms of MSD) but significant increase in telomere mobility was observed. The effects were less dramatic than expected, which can be attributed to the heterogeneity in LMNA knockdown level between cells. Another possibility is that several transfected cells had not (yet) undergone mitosis and, given the slow turnover rate of lamin A [6], still contained a functional lamina. In our hands, transfected cells often encountered difficulties proceeding through mitosis, which also explains our current inability to visualize a 'rescue' phenotype in $\mathrm{HDF}^{\mathrm{mut} / \mathrm{mut}}$ after transfection with pEGFP-LaminA. 
Similar findings were reported previously in mouse embryonic fibroblasts at high passage number [70].

\section{Lower telomere mobility in HGPS cells can be increased by FTI-treatment}

In progeria cells, telomere movement appeared more restricted. From one point of view, this was an unexpected finding since progeria cells have shorter telomeres [71] and recently it was shown that shorter or damaged telomeres experience increased telomere mobility, possibly to increase probability of finding repair or elongation mechanisms $[52,72,73]$. On the other hand, there can be substantial differences in telomere length between patients, making this a double-edged sword and therefore demands caution. In cells from this HGPS patient, nuclei were generally smaller, which itself is a significant restraining factor. The slower mobility rates in HGPS cells might also be a reflection of the increased intranuclear pressure and altered internal topography due to accumulation of progerin. At the same time progerin interacts with several proteins and chromatin. It is conceivable that this mutated lamin precursor exerts a (non-)specific inertia, creating increased intranuclear viscosity and thereby hampering normal locomotion and thus function. Indeed, treatment of HGPS cells with FTI revealed an increase in telomere mobility again pointing to a potentially more general role for lamin A processing in intranuclear dynamics.

In conclusion, using light-economical and quantitative microscopy techniques, we revealed a direct correlation between increased nuclear and telomere dynamics and the loss of A-type lamin expression. Conversely, lamina stiffening due to abnormal lamin $\mathrm{A} / \mathrm{C}$ processing leads to decreased nuclear dynamics. To obtain more general data and increase the power of our findings, these experiments should be expanded to more cell types of more patients. Nevertheless, given the fact that we were able to experimentally alter phenotypes by manipulating either LMNA expression levels or processing, our observations indicate a prominent role for lamins in nuclear dynamics. We observed marked differences in evolution of nuclear shape with culture age (generation dynamics) and on the level of the single cell, we found a varying degree of nuclear deformation (nuclear membrane dynamics) and distinct telomere mobility regimes (subnuclear dynamics). Because of the highly organized and compartmentalized character of the nucleus, altered intranuclear dynamics could have serious consequences for cellular function: repositioning of gene loci or failure to do so may 
influence gene expression and regulation and could contribute to genome instability [1, $3,17,68]$. Therefore these differences could portray important novel mechanisms of disease for laminopathies.

\section{Acknowledgements}

We thank Ir. Thomas Martens for his excellent imaging work, Dr.

Tim De Meyer from the Laboratory of Bioinformatics and Computational

Genomics (Ghent University) for his expert assistance with

statistical analysis and Prof. Kevin Braeckmans from the Laboratory of

General Biochemistry and Physical Pharmacy (Ghent University) for

his advice on mobility modelling and analysis.

\section{Video}

Video 1:

http://dl.dropbox.com/u/37827993/Chapter3-video1.mov

Video 2:

http://dl.dropbox.com/u/37827993/Chapter3-video2.mov

Video 3:

http://dl.dropbox.com/u/37827993/Chapter3-video3.mov 


\section{References}

[1] T. Misteli, Concepts in nuclear architecture, Bioessays 27 (2005) 477-487.

[2] D.L. Spector, Nuclear domains, J Cell Sci 114 (2001) 2891-2893.

[3] T. Cremer and C. Cremer, Chromosome territories, nuclear architecture and gene regulation in mammalian cells, Nat Rev Genet 2 (2001) 292-301.

[4] T. Shimi, K. Pfleghaar, S. Kojima, C.G. Pack, I. Solovei, A.E. Goldman, S.A. Adam, D.K. Shumaker, M. Kinjo, T. Cremer and R.D. Goldman, The A- and B-type nuclear lamin networks: microdomains involved in chromatin organization and transcription, Genes Dev 22 (2008) 3409-3421.

[5] A.E. Goldman, R.D. Moir, M. Montag-Lowy, M. Stewart and R.D. Goldman, Pathway of incorporation of microinjected lamin A into the nuclear envelope, J Cell Biol 119 (1992) 725-735.

[6] J.L. Broers, B.M. Machiels, G.J. van Eys, H.J. Kuijpers, E.M. Manders, R. van Driel and F.C. Ramaekers, Dynamics of the nuclear lamina as monitored by GFP-tagged A-type lamins, J Cell Sci 112 ( Pt 20) (1999) 3463-3475.

[7] R.D. Goldman, Y. Gruenbaum, R.D. Moir, D.K. Shumaker and T.P. Spann, Nuclear lamins: building blocks of nuclear architecture, Genes Dev 16 (2002) 533-547.

[8] J.L. Broers, F.C. Ramaekers, G. Bonne, R.B. Yaou and C.J. Hutchison, Nuclear lamins: laminopathies and their role in premature ageing, Physiol Rev 86 (2006) 967-1008.

[9] J.L. Broers, C.J. Hutchison and F.C. Ramaekers, Laminopathies, J Pathol 204 (2004) 478-488.

[10] H.J. Worman and G. Bonne, "Laminopathies": a wide spectrum of human diseases, Exp Cell Res 313 (2007) 2121-2133.

[11] C.L. Navarro, J. Cadinanos, A. De Sandre-Giovannoli, R. Bernard, S. Courrier, I. Boccaccio, A. Boyer, W.J. Kleijer, A. Wagner, F. Giuliano, F.A. Beemer, J.M. Freije, P. Cau, R.C. Hennekam, C. Lopez-Otin, C. Badens and N. Levy, Loss of ZMPSTE24 (FACE-1) causes autosomal recessive restrictive dermopathy and accumulation of Lamin A precursors, Hum Mol Genet 14 (2005) 1503-1513.

[12] M. Eriksson, W.T. Brown, L.B. Gordon, M.W. Glynn, J. Singer, L. Scott, M.R. Erdos, C.M. Robbins, T.Y. Moses, P. Berglund, A. Dutra, E. Pak, S. Durkin, A.B. Csoka, M. Boehnke, T.W. Glover and F.S. Collins, Recurrent de novo point mutations in lamin A cause Hutchinson-Gilford progeria syndrome, Nature 423 (2003) 293-298.

[13] C.L. Navarro, P. Cau and N. Levy, Molecular bases of progeroid syndromes, Hum Mol Genet 15 Spec No 2 (2006) R151-161.

[14] K. Cao, B.C. Capell, M.R. Erdos, K. Djabali and F.S. Collins, A lamin A protein isoform overexpressed in Hutchinson-Gilford progeria syndrome interferes with mitosis in progeria and normal cells, Proc Natl Acad Sci U S A 104 (2007) 4949-4954. 
[15] M.W. Glynn and T.W. Glover, Incomplete processing of mutant lamin A in Hutchinson-Gilford progeria leads to nuclear abnormalities, which are reversed by farnesyltransferase inhibition, Hum Mol Genet 14 (2005) 29592969.

[16] R.D. Goldman, D.K. Shumaker, M.R. Erdos, M. Eriksson, A.E. Goldman, L.B. Gordon, Y. Gruenbaum, S. Khuon, M. Mendez, R. Varga and F.S. Collins, Accumulation of mutant lamin A causes progressive changes in nuclear architecture in Hutchinson-Gilford progeria syndrome, Proc Natl Acad Sci U S A 101 (2004) 8963-8968.

[17] K.L. Reddy, J.M. Zullo, E. Bertolino and H. Singh, Transcriptional repression mediated by repositioning of genes to the nuclear lamina, Nature 452 (2008) 243-247.

[18] K.N. Dahl, A.J. Ribeiro and J. Lammerding, Nuclear shape, mechanics, and mechanotransduction, Circ Res 102 (2008) 1307-1318.

[19] K.N. Dahl, P. Scaffidi, M.F. Islam, A.G. Yodh, K.L. Wilson and T. Misteli, Distinct structural and mechanical properties of the nuclear lamina in Hutchinson-Gilford progeria syndrome, Proc Natl Acad Sci U S A 103 (2006) 10271-10276.

[20] J. Lammerding, P.C. Schulze, T. Takahashi, S. Kozlov, T. Sullivan, R.D. Kamm, C.L. Stewart and R.T. Lee, Lamin A/C deficiency causes defective nuclear mechanics and mechanotransduction, J Clin Invest 113 (2004) 370378.

[21] T. Sullivan, D. Escalante-Alcalde, H. Bhatt, M. Anver, N. Bhat, K. Nagashima, C.L. Stewart and B. Burke, Loss of A-type lamin expression compromises nuclear envelope integrity leading to muscular dystrophy, J Cell Biol 147 (1999) 913-920.

[22] J.L. Broers, E.A. Peeters, H.J. Kuijpers, J. Endert, C.V. Bouten, C.W. Oomens, F.P. Baaijens and F.C. Ramaekers, Decreased mechanical stiffness in LMNA-/- cells is caused by defective nucleo-cytoskeletal integrity: implications for the development of laminopathies, Hum Mol Genet 13 (2004) 2567-2580.

[23] V.L. Verstraeten, J.Y. Ji, K.S. Cummings, R.T. Lee and J. Lammerding, Increased mechanosensitivity and nuclear stiffness in Hutchinson-Gilford progeria cells: effects of farnesyltransferase inhibitors, Aging Cell 7 (2008) 383-393.

[24] M. Crisp, Q. Liu, K. Roux, J.B. Rattner, C. Shanahan, B. Burke, P.D. Stahl and D. Hodzic, Coupling of the nucleus and cytoplasm: role of the LINC complex, J Cell Biol 172 (2006) 41-53.

[25] J.Y. Ji, R.T. Lee, L. Vergnes, L.G. Fong, C.L. Stewart, K. Reue, S.G. Young, Q. Zhang, C.M. Shanahan and J. Lammerding, Cell nuclei spin in the absence of lamin b1, J Biol Chem 282 (2007) 20015-20026.

[26] C.M. Hale, A.L. Shrestha, S.B. Khatau, P.J. Stewart-Hutchinson, L. Hernandez, C.L. Stewart, D. Hodzic and D. Wirtz, Dysfunctional connections 
between the nucleus and the actin and microtubule networks in laminopathic models, Biophys J 95 (2008) 5462-5475.

[27] P.J. Stewart-Hutchinson, C.M. Hale, D. Wirtz and D. Hodzic, Structural requirements for the assembly of LINC complexes and their function in cellular mechanical stiffness, Exp Cell Res 314 (2008) 1892-1905.

[28] L. Mounkes, S. Kozlov, B. Burke and C.L. Stewart, The laminopathies: nuclear structure meets disease, Curr Opin Genet Dev 13 (2003) 223-230.

[29] T.P. Spann, R.D. Moir, A.E. Goldman, R. Stick and R.D. Goldman, Disruption of nuclear lamin organization alters the distribution of replication factors and inhibits DNA synthesis, J Cell Biol 136 (1997) 1201-1212.

[30] D.K. Shumaker, T. Dechat, A. Kohlmaier, S.A. Adam, M.R. Bozovsky, M.R. Erdos, M. Eriksson, A.E. Goldman, S. Khuon, F.S. Collins, T. Jenuwein and R.D. Goldman, Mutant nuclear lamin A leads to progressive alterations of epigenetic control in premature aging, Proc Natl Acad Sci U S A 103 (2006) 8703-8708.

[31] K.J. Meaburn, E. Cabuy, G. Bonne, N. Levy, G.E. Morris, G. Novelli, I.R. Kill and J.M. Bridger, Primary laminopathy fibroblasts display altered genome organization and apoptosis, Aging Cell 6 (2007) 139-153.

[32] V. Ondrej, E. Lukasova, J. Krejci, P. Matula and S. Kozubek, Lamin A/C and polymeric actin in genome organization, Mol Cells 26 (2008) 356-361.

[33] G. Galiova, E. Bartova, I. Raska, J. Krejci and S. Kozubek, Chromatin changes induced by lamin $\mathrm{A} / \mathrm{C}$ deficiency and the histone deacetylase inhibitor trichostatin A, Eur J Cell Biol 87 (2008) 291-303.

[34] A. Mattout-Drubezki and Y. Gruenbaum, Dynamic interactions of nuclear lamina proteins with chromatin and transcriptional machinery, Cell Mol Life Sci 60 (2003) 2053-2063.

[35] R.L. Shoeman and P. Traub, The in vitro DNA-binding properties of purified nuclear lamin proteins and vimentin, J Biol Chem 265 (1990) 9055-9061.

[36] L. Guelen, L. Pagie, E. Brasset, W. Meuleman, M.B. Faza, W. Talhout, B.H. Eussen, A. de Klein, L. Wessels, W. de Laat and B. van Steensel, Domain organization of human chromosomes revealed by mapping of nuclear lamina interactions, Nature 453 (2008) 948-951.

[37] A. Pyrpasopoulou, J. Meier, C. Maison, G. Simos and S.D. Georgatos, The lamin B receptor (LBR) provides essential chromatin docking sites at the nuclear envelope, Embo J 15 (1996) 7108-7119.

[38] T. Dechat, A. Gajewski, B. Korbei, D. Gerlich, N. Daigle, T. Haraguchi, K. Furukawa, J. Ellenberg and R. Foisner, LAP2alpha and BAF transiently localize to telomeres and specific regions on chromatin during nuclear assembly, J Cell Sci 117 (2004) 6117-6128.

[39] T. de Lange, Human telomeres are attached to the nuclear matrix, Embo J 11 (1992) 717-724.

[40] K. Weipoltshammer, C. Schofer, M. Almeder, V.V. Philimonenko, K. Frei, F. Wachtler and P. Hozak, Intranuclear anchoring of repetitive DNA sequences: 
centromeres, telomeres, and ribosomal DNA, J Cell Biol 147 (1999) 14091418.

[41] J. Schmitt, R. Benavente, D. Hodzic, C. Hoog, C.L. Stewart and M. Alsheimer, Transmembrane protein Sun2 is involved in tethering mammalian meiotic telomeres to the nuclear envelope, Proc Natl Acad Sci U S A 104 (2007) 7426-7431.

[42] H. Schober, H. Ferreira, V. Kalck, L.R. Gehlen and S.M. Gasser, Yeast telomerase and the SUN domain protein Mps3 anchor telomeres and repress subtelomeric recombination, Genes Dev 23 (2009) 928-938.

[43] W.H. De Vos, R.A. Hoebe, G.H. Joss, W. Haffmans, S. Baatout, P. Van Oostveldt and E.M. Manders, Controlled light exposure microscopy reveals dynamic telomere microterritories throughout the cell cycle, Cytometry A 75 (2009) 428-439.

[44] R.A. Hoebe, C.H. Van Oven, T.W. Gadella, Jr., P.B. Dhonukshe, C.J. Van Noorden and E.M. Manders, Controlled light-exposure microscopy reduces photobleaching and phototoxicity in fluorescence live-cell imaging, Nat Biotechnol 25 (2007) 249-253.

[45] R.A. Hoebe, H.T. Van der Voort, J. Stap, C.J. Van Noorden and E.M. Manders, Quantitative determination of the reduction of phototoxicity and photobleaching by controlled light exposure microscopy, J Microsc 231 (2008) 9-20.

[46] W.S. Rasband, ImageJ, U.S. National Institutes of Health, Bethesda, Maryland, USA, http://rsb.info.nih.gov/ij, 1997-2009.

[47] B. Hebert, S. Costantino and P.W. Wiseman, Spatiotemporal image correlation spectroscopy (STICS) theory, verification, and application to protein velocity mapping in living CHO cells, Biophys J 88 (2005) 3601-3614.

[48] E.M. Manders, J. Stap, G.J. Brakenhoff, R. van Driel and J.A. Aten, Dynamics of three-dimensional replication patterns during the S-phase, analysed by double labelling of DNA and confocal microscopy, J Cell Sci 103 ( Pt 3) (1992) 857-862.

[49] W.H. De Vos, G.H. Joss, W. Haffmans, R.A. Hoebe, E.M. Manders and P. Van Oostveldt, Four-dimensional telomere analysis in recordings of living human cells acquired with controlled light exposure microscopy, J Microsc 238 254-264.

[50] H. Bornfleth, P. Edelmann, D. Zink, T. Cremer and C. Cremer, Quantitative motion analysis of subchromosomal foci in living cells using four-dimensional microscopy, Biophys J 77 (1999) 2871-2886.

[51] M.J. Saxton, Lateral diffusion in an archipelago. Single-particle diffusion, Biophys J 64 (1993) 1766-1780.

[52] T. Jegou, I. Chung, G. Heuvelman, M. Wachsmuth, S.M. Gorisch, K.M. Greulich-Bode, P. Boukamp, P. Lichter and K. Rippe, Dynamics of telomeres and promyelocytic leukemia nuclear bodies in a telomerase-negative human cell line, Mol Biol Cell 20 (2009) 2070-2082. 
[53] D.R.C. G.E.P. Box, An Analysis of Transformations, J. R. Stat. Soc. Ser. BStat. Method. 26 (1964) 6631.

[54] C. Molenaar, K. Wiesmeijer, N.P. Verwoerd, S. Khazen, R. Eils, H.J. Tanke and R.W. Dirks, Visualizing telomere dynamics in living mammalian cells using PNA probes, Embo J 22 (2003) 6631-6641.

[55] J.R. Chubb, S. Boyle, P. Perry and W.A. Bickmore, Chromatin motion is constrained by association with nuclear compartments in human cells, Curr Biol 12 (2002) 439-445.

[56] S.M. Gorisch, M. Wachsmuth, C. Ittrich, C.P. Bacher, K. Rippe and P. Lichter, Nuclear body movement is determined by chromatin accessibility and dynamics, Proc Natl Acad Sci U S A 101 (2004) 13221-13226.

[57] J. Lammerding, L.G. Fong, J.Y. Ji, K. Reue, C.L. Stewart, S.G. Young and R.T. Lee, Lamins A and C but not lamin B1 regulate nuclear mechanics, J Biol Chem 281 (2006) 25768-25780.

[58] R.I. Kumaran and D.L. Spector, A genetic locus targeted to the nuclear periphery in living cells maintains its transcriptional competence, J Cell Biol 180 (2008) 51-65.

[59] J.S. Lee, C.M. Hale, P. Panorchan, S.B. Khatau, J.P. George, Y. Tseng, C.L. Stewart, D. Hodzic and D. Wirtz, Nuclear lamin A/C deficiency induces defects in cell mechanics, polarization, and migration, Biophys J 93 (2007) 2542-2552.

[60] S.B. Khatau, C.M. Hale, P.J. Stewart-Hutchinson, M.S. Patel, C.L. Stewart, P.C. Searson, D. Hodzic and D. Wirtz, A perinuclear actin cap regulates nuclear shape, Proc Natl Acad Sci U S A 106 (2009) 19017-19022.

[61] U. Aebi, J. Cohn, L. Buhle and L. Gerace, The nuclear lamina is a meshwork of intermediate-type filaments, Nature 323 (1986) 560-564.

[62] R.I. Kumaran, B. Muralikrishna and V.K. Parnaik, Lamin A/C speckles mediate spatial organization of splicing factor compartments and RNA polymerase II transcription, J Cell Biol 159 (2002) 783-793.

[63] W.F. Marshall, A. Straight, J.F. Marko, J. Swedlow, A. Dernburg, A. Belmont, A.W. Murray, D.A. Agard and J.W. Sedat, Interphase chromosomes undergo constrained diffusional motion in living cells, Curr Biol 7 (1997) 930-939.

[64] J. Vazquez, A.S. Belmont and J.W. Sedat, Multiple regimes of constrained chromosome motion are regulated in the interphase Drosophila nucleus, Curr Biol 11 (2001) 1227-1239.

[65] Y. Tseng, J.S. Lee, T.P. Kole, I. Jiang and D. Wirtz, Micro-organization and visco-elasticity of the interphase nucleus revealed by particle nanotracking, $\mathrm{J}$ Cell Sci 117 (2004) 2159-2167.

[66] M.E. Luderus, A. de Graaf, E. Mattia, J.L. den Blaauwen, M.A. Grande, L. de Jong and R. van Driel, Binding of matrix attachment regions to lamin B1, Cell 70 (1992) 949-959.

[67] K. Prufert, A. Vogel and G. Krohne, The lamin CxxM motif promotes nuclear membrane growth, J Cell Sci 117 (2004) 6105-6116. 
[68] I. Gonzalez-Suarez, A.B. Redwood, S.M. Perkins, B. Vermolen, D. Lichtensztejin, D.A. Grotsky, L. Morgado-Palacin, E.J. Gapud, B.P. Sleckman, T. Sullivan, J. Sage, C.L. Stewart, S. Mai and S. Gonzalo, Novel roles for A-type lamins in telomere biology and the DNA damage response pathway, Embo J 28 (2009) 2414-2427.

[69] E. Soutoglou and T. Misteli, Mobility and immobility of chromatin in transcription and genome stability, Curr Opin Genet Dev 17 (2007) 435-442.

[70] J.L. Broers, H.J. Kuijpers, C. Ostlund, H.J. Worman, J. Endert and F.C. Ramaekers, Both lamin A and lamin C mutations cause lamina instability as well as loss of internal nuclear lamin organization, Exp Cell Res 304 (2005) 582-592.

[71] R.C. Allsopp, H. Vaziri, C. Patterson, S. Goldstein, E.V. Younglai, A.B. Futcher, C.W. Greider and C.B. Harley, Telomere length predicts replicative capacity of human fibroblasts, Proc Natl Acad Sci U S A 89 (1992) 1011410118.

[72] X. Wang, Z. Kam, P.M. Carlton, L. Xu, J.W. Sedat and E.H. Blackburn, Rapid telomere motions in live human cells analyzed by highly time-resolved microscopy, Epigenetics Chromatin 1 (2008) 4.

[73] N. Dimitrova, Y.C. Chen, D.L. Spector and T. de Lange, 53BP1 promotes non-homologous end joining of telomeres by increasing chromatin mobility, Nature 456 (2008) 524-528. 


\section{Chapter 4}

\section{Repetitive Disruptions of the Nuclear Envelope Invoke Temporary Loss of Cellular Compartmentalization in Laminopathies}

W.H. De Vos*, F. Houben*, M.A. Kamps, A. Malhas, F. Verheyen, J. Cox, E.M.M. Manders, V.L.R.M. Verstraeten, M.A.M. van Steensel, C.L.M. Marcelis, A. van den Wijngaard, D.J. Vaux, F.C.S. Ramaekers, J.L.V. Broers

Published in part in:

Human Molecular Genetics; 20 (2011) 4175-4186

* Both authors contributed equally to this chapter 


\begin{abstract}
The nuclear lamina provides structural support to the nucleus and has a central role in nuclear organization and gene regulation. Defects in its constituents, the lamins, lead to a class of genetic diseases collectively referred to as laminopathies. Using live cell imaging, we observed the occurrence of intermittent, non-lethal ruptures of the nuclear envelope in dermal fibroblast cultures of patients with different mutations of lamin $\mathrm{A} / \mathrm{C}$.

These ruptures, which were absent in normal fibroblasts, could be mimicked by selective knockdown as well as knockout of $L M N A$ and were accompanied by the loss of cellular compartmentalization. This was demonstrated by the influx of cytoplasmic transcription factor RelA and regulatory protein Cyclin B1 into the nucleus, and efflux of nuclear transcription factor OCT1 and nuclear PML-containing structures to the cytoplasm.

While recovery of EYFP-tagged nuclear localization signal in the nucleus demonstrated restoration of nuclear membrane integrity, part of the mobile PML structures became permanently translocated to the cytoplasm. These satellite PML structures were devoid of the typical PML body components such as DAXX, SP100 or SUMO1. Our data suggest that nuclear rupture and loss of compartmentalization may add to cellular dysfunction and disease development in various laminopathies.
\end{abstract}




\section{Introduction}

The nuclear envelope is the principal barrier dictating bidirectional communication between the nucleus and cytoplasm of the cell [1]. Directly underlying the double lipid bilayer is an intimately connected meshwork of intermediate filament proteins, the nuclear lamina, which provides structural support to the nucleus and has a central role in defining nuclear organization [2]. Defects in one of its major constituents, the Atype nuclear lamins, lead to a spectrum of diseases collectively referred to as laminopathies.

Disease manifestations range from systemic premature aging, such as observed in Hutchinson-Gilford Progeria Syndrome (HGPS), to muscular dystrophies and lipodystrophies [3]. One hypothesis for explaining disease development premises that gene regulation becomes affected due to disturbed interactions between mutant A-type lamins and chromatin or transcription factors [4]. Another frequently postulated hypothesis argues that mutations in the LMNA gene cause mechanical weakening of the lamina and nuclear envelope, leading to accumulation of nuclear damage under physical stress conditions $[5,6]$. While both theories are supported by experimental data, so far no conclusive mechanism for the development of laminopathies has been established.

It is widely accepted that the nuclear membrane becomes dramatically affected in laminopathy cells; dilation of the perinuclear space [7], aggregation of nuclear pore complexes [8] and even complete local absence of an overt lamina $[9,10]$ have been documented both in cell cultures and in patient tissues. However, little is known about the implications of these structural alterations of the nuclear envelope for nucleocytoplasmic communication. While it has been shown that nuclear transport becomes altered in cell lines over-expressing specific mutant (pre-)lamin A isoforms [11], there is no clear view on the general impact of LMNA mutations on cellular compartmentalization. Here, we demonstrate that different mutations of A-type lamins cause intermittent, non-lethal ruptures of the nuclear envelope, which lead to temporary exchange of proteins between nucleus and cytoplasm, as well as permanent translocation of nuclear protein complexes to the cytoplasm and vice versa. The potential impact of this loss of cellular compartmentalization on disease development is discussed. 


\section{Material \& Methods}

\section{Cell cultures}

The following human dermal fibroblast cultures from healthy volunteers and patients were used in this study: 1) Two different batches of normal human dermal fibroblasts (NHDFa, $+/+$, Promocell, Heidelberg, Germany); 2) Cells from a patient with a lethal laminopathy phenotype due to a nonsense Y259X homozygous mutation in the LMNA gene (double-null, Y259X/Y259X) [12]; 3) Hutchinson-Gilford Progeria Syndrome cells (HGPS, +/G608G) [13]; 4) Cells from a compound progeroid syndrome patient, carrying a LMNA M540T and a LMNA T528M mutation on different alleles (M540T/T528M) as well as cells from the patients' father and mother, who have no disease symptoms [13]. The apparently healthy father carries the c.1583C.T (p.T528M) mutation in exon $9(+/ \mathrm{T} 528 \mathrm{M})$. This mutation is associated in literature with FPLD [14]. The mother carries the c.1619T.C (p.M540T) mutation in exon 10 and is also symptom-free (+/M540T); 5) Cells from a patient with familial partial lipodystrophy (FPLD) carrying a R439C mutation in the LMNA gene (+/R439C) [15]; 6) cells from a patient suffering from restrictive dermopathy (RD), with homozygous c.1085_1086insT truncating mutations in the ZMPSTE24 gene. This frameshift mutation leads to a premature termination codon of the protein and inactivation of the ZMPSTE24 enzyme, resulting in prelamin A accumulation in patient nuclei [16]; 7) Cells from a recently diagnosed patient with hypertrophic cardiomyopathy (HCM) with a defect in lamin A only (+/R644C). Informed consent for inclusion in this study was obtained from the patients or from their parents. In addition, NIH-3T3 mouse fibroblasts, wild-type cells and cells with stable lamin A/C knock-down [17] as well as wild type and lamin A/C knockout mouse embryonic fibroblasts (MEFs) (Lmna-/-) were used [18]. Cells were cultured in Advanced DMEM/F12 medium (Gibco, Invitrogen, Merelbeke, Belgium) supplemented with $10 \%$ fetal calf serum and $1 \%$ penicilin/streptomycin/glutamin, at $37^{\circ} \mathrm{C}$ and $5 \% \mathrm{CO} 2$, according to standard procedures. In case of direct comparison, passage-matched cells were used.

\section{Transfection}

The following constructs were used for transfection: EYFP-NLS or mCherry-NLS [19] (generous gifts from Dr. J. Goedhart, University of Amsterdam, the Netherlands), EGFPLMNB, GFP-OCT1 [20], EGFP-NUP153 [21] (a kind gift of Birthe Fahrenkrog, Universite Libre de Bruxelles, Brussels, Belgium), GFPRelA (Addgene plasmid 
23255) [22], and/or with EYFP-PML [23] (a kind gift from Dr. J. Wiegant, Leiden University, the Netherlands), Cyclin B1-GFP [24, 25] (kindly provided by Dr. J. Pines, Cambridge, U.K) and EGFP-D50-Lamin A (Addgene plasmid 17653) [26]. For transfection, GeneJammer (Stratagene, La Jolla, California, USA) or Lipofectamine 2000 (Invitrogen, Merelbeke, Belgium) was used, according to the manufacturer's instructions.

\section{Live cell imaging}

Time-lapse recordings were made $24 \mathrm{~h}-48 \mathrm{~h}$ after transfection as described previously $[17,27]$. Care was taken to only select cells with moderate expression levels and correct (initial) localization patterns. At least 3 different biological replicates were used. Cellular condition was also verified by phase contrast microscopy, to assure that cells showed a normal morphology without excessive vacuole formation. Live cell imaging was performed on a Nikon A1R confocal microscope, mounted on a Nikon Ti body, equipped with a Perfect Focus System and a microscope incubator equilibrated at $36.5^{\circ} \mathrm{C}$.

Recordings were made using a 60x/1.4 Plan Apo oil immersion lens. Alternatively, live cell imaging was performed on an inverted fluorescence microscope (Leica DIRBE, Leica Microsytems BV, Rijswijk, The Netherlands), equipped with a black and white CCD camera (CA4742-95, Hamamatsu), a polychrome II polychromator as light source for fluorescence (TILL Photonics, Martinsried, Germany) and a 20x/0.7 Plan Apo lens.

Image acquisition was achieved using appropriate software, while image (pre)processing, analysis and annotation was performed in ImageJ freeware v1.43 [28], Adobe Photoshop ${ }^{\circledR}$ 9.0.2 and Adobe Illustrator ${ }^{\circledR}$ 12.0.1 (Adobe Systems Inc., CA, USA).

\section{Immunofluorescence labeling}

Human fibroblasts were grown, fixed and immunostained as previously described [17] using the following antibodies: PML (PG-M3) mouse monoclonal antibody (1:1000, Sc-966, Santa Cruz, Heidelberg, Germany); Lamin A/C mouse monoclonal antibody Jol2 (1:50, kindly provided by Prof. C. Hutchison, Durham, UK); Lamin B rabbit polyclonal antibody (1:500, ab16048, Abcam, Cambridge, UK); Lamin B (C-20) goat polyclonal antibody (1:500, sc-6216, Santa Cruz, Heidelberg, Germany); MAB414 mouse monoclonal antibody to NUP153 (1:2000, ab24609, Abcam, Cambridge, UK), DAXX rabbit polyclonal antibody (1:200, HPA008736, Sigma-Aldrich, St. Louis, MO); SP100 rabbit polyclonal antibody (1:200, HPA016707, Sigma-Aldrich, St. Louis, 
MO); SUMO1 rabbit polyclonal antibody (1:200, ab32058, Abcam, Cambridge, UK). As secondary antibodies FITC-conjugated goat anti-mouse Ig (1:50, SBA/ITK Birmingham, AL, USA), Texas Red conjugated goat anti-rabbit Ig (1:50, SBA/ITK), Dylight 488 donkey anti-mouse IgG (1:300, 715-486-150 Jackson Immunoresearch Laboratories, PA, USA), Dylight 549 donkey anti-rabbit IgG (1:300, 711-506-152 Jackson Immunoresearch Laboratories, PA, USA) and Dylight 649 donkey anti-goat IgG (1:300, 705-496-147 Jackson Immunoresearch Laboratories, PA, USA) were used. Immunostained cells were mounted in Vectashield (Invitrogen, Merelbeke, Belgium) containing $1 \mathrm{ng} / \mathrm{ml}$ DAPI and visualized using a Leica TCS SPE confocal laser scanning fluorescence microscope (Leica DMRBE, Mannheim, Germany) using LASAF software (version 2.2, Leica) or with a Nikon TE2000 Eclipse widefield microscope.

\section{Dextran scrape loading}

Passage-matched cells (between passage 10-15) were treated using a variation of the scrape loading technique [29]. Cells, grown on coverslips to near-confluency, were submerged in culture medium containing $1 \mathrm{mg} / \mathrm{ml}$ Dextran-Texas Red (70kD, Molecular Probes, Oregon). Using a blunted hypodermic needle the glass surface was scratched and wounded cells were allowed to take up dextran for 5 minutes. While severely damaged cells at the central region of the wound area in general detached, at least 300 viable, attached cells containing dextran could be visualized using this technique and were scored on a $25 \mathrm{~mm}$ round coverslip. For each experiment 3 coverslips were used per time point, and all experiments were repeated at least 3 times. Next, cells were washed three times with medium and fixed with $3.7 \%$ formaldehyde in PBS after variable incubation periods $(0,4,8$, and 24 hours). Nuclei were counterstained with DAPI and the presence of intranuclear dextran was assessed by analyzing confocal z-series.

\section{Image Analysis}

For scoring of nuclear shape aberrations or cytoplasmic PML bodies, either a high content analysis was performed as described before [30], or scored manually. Nuclear aberrations were identified as honeycomb structures after lamin labeling or nuclear herniations and holes after DAPI staining. In scoring of PML bodies, a second nonspecific channel was acquired (550LP) to prevent autofluorescent foci from skewing the analysis. Mitotic and early G1 cells, which may exhibit some cytoplasmic PML bodies, were rejected from the analysis based on intensity and shape criteria of the DAPI staining. 


\section{Electron Microscopy}

LMNA double-null cells (Y259X/Y259X) were cultured for at least 48 hours in a culture dish after which the medium was discarded. Next, the cells were shortly rinsed with PBS and subsequently fixed at room temperature with $3 \%$ glutaraldehyde in $0.1 \mathrm{M}$ cacodylate buffer ( $\mathrm{pH}$ 7.4) supplemented with $0.5 \mathrm{mM} \mathrm{CaCl} 2$ for at least $24 \mathrm{hrs}$. The cells were then postfixed with $2 \%$ OsO4 in cacodylate buffer (pH 7.4) containing 1.5 $\%$ potassium ferricyanide at $4{ }^{\circ} \mathrm{C}$ for one hr. After a short rinse in cacodylate buffer, the cells were further dehydrated in graded ethanol series before embedding in epon. Ultra thin sections were stained with uranyl acetate and lead citrate and examined in a Philips CM100 electron microscope at $80 \mathrm{KV}$.

\section{Real-time PCR}

Wild type and Lmna-/- MEFs or wild type cells were seeded at equal densities and harvested the day after, at about $75 \%$ confluency for RNA extraction. RNA was extracted from 5 biological replicates for each cell type, using High Pure RNA Isolation Kit (Roche, West Sussex, UK), according to the manufacturer's instructions. Superscript III reverse transcriptase (Invitrogen, Paisley, UK) was used for first strand cDNA synthesis. Real-time PCR was performed using an Applied Biosystems Step One Plus apparatus and the FAST SYBR GREEN MasterMix (Applied Biosystems, CA, USA). Relative gene expression values were determined using the $2-\mathrm{Ct}$ method [31], using the housekeeping gene $\beta$-actin (Actb, forward primer: ACTCCTATGTGGGTGACGAG, reverse primer: CCAGATCTTCTCCATGTCGT) as an internal standard and Lmna (forward: GCAACAAGTCCAATGAGGACCA, reverse: GTCCCAGATTACATGATGC ) as an internal control. The following primer pairs were used for Oct1-responsive target genes: $\operatorname{Prdx2}$ (forward: TGGGCTTGATGGTGTCACT, reverse: CGTCTGTAGACGAGGCTCT), Gas5 (forward: CGCTCTGTTATC-CAGCTTCC, reverse: AGCTGTGCGGCATTCTGAG, Sod1 (forward: TACTGATGGACGTGGAACCC, reverse: GAACCATCCACTTCGAGCA), Gpx3 (forward: GCAACAAGTCCAATGAGGACCA, reverse: GTCCCAGA-TTACATGATGC), Serping1 (forward: AAGTTGGTGCTTTGGGAACA, reverse: GCCCAATTCGATGACCATAC), Mmp13 (forward: GGTCCTTGGAGTGATCCAGA, reverse: TGATGAAACCTGGACAAGCA) Il6 (forward: AAGTCCGGAGAGACTTC, reverse: CAAGTGCATCATCGT-TGTTC), $\quad$ grst (forward: TGAAAACATCGTTCCCTTTC, reverse: GGAAGGGGAGTCAAGTAAGC), $R d m 1$ (forward: TGGCCTTCTGTATTCAGTCC, reverse: CAAGACGAACCTTCACTGGT). 


\section{Results}

\section{$70 \mathrm{kD}$ dextran particles accumulate in the nucleus of laminopathy cells}

Primary fibroblasts (all between passage number 10 and 15), derived from different laminopathy patients, were scrape-loaded with Texas Red-labeled dextran (MW $>70 \mathrm{kDa}$ ), which should normally not pass the nuclear pores of an intact nuclear envelope (Fig. 1a). Using confocal microscopy, we assessed cellular dextran distribution at different time points after loading cells of a progeroid syndrome patient, carrying a compound heterozygous p.M540T and p.T528M missense mutation on different alleles of the LMNA gene (M540T/T528M), as well as in cells from a patient with a lethal nonsense Y259X homozygous mutation in LMNA (Y259X/Y259X). For comparison, we used passage-matched scrape loaded dermal fibroblast cells (NHDF $\alpha$, $+/+$ ) from a healthy donor as a control. Immediately after scrape-loading no living (attached) control cells containing cytoplasmic dextran could be detected with intranuclear dextran.
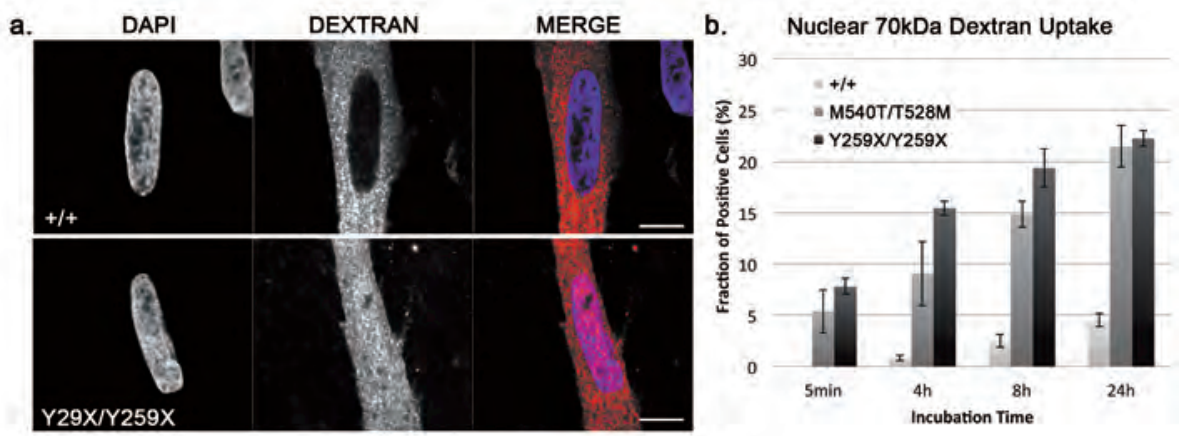

Figure 1. Nuclear uptake of $70 \mathrm{kD}$ dextran after scrape loading. 70kDa Dextran-Texas Red was added to a confluent cell culture, followed by scrape loading using a blunted hypodermic needle. After 5 minutes dextran was removed and cells were allowed to recover. Cells were fixed at different time points after loading $(0,4,8$, and 24 hours), and scored for nucleoplasmic dextran accumulation using confocal microscopy after DAPI staining. (a) Confocal images $4 \mathrm{~h}$ after dextran labeling. The top panel shows a control fibroblast in which the nucleus is largely devoid of dextran signal. Isolated faint nuclear spots of dextran probably represent dextran in transnuclear channels of the nucleoplasmic reticulum. The bottom panel demonstrates prominent uptake of dextran into the nucleus of a Y259X/Y259X cell. The scale bar represents $10 \mu \mathrm{m}$. (b) Histogram showing the percentages of cells with nuclear dextran uptake in different cultures at indicated time points after scrape loading. At all time points the number of dextran positive nuclei was significantly higher for M540T/T528M and Y259X/Y259X cells compared to control $+/+$ cells (t-test, p-value $<1 \mathrm{E}-05)$. 
During the first 24 hours after scrape-loading, laminopathy cells showed a significantly and progressively higher nuclear uptake of fluorescent dextran molecules compared to control cells (Fig. 1b). After 24 hours, only $4.6 \pm 0.6 \%$ of the control $+/+$ cells showed nuclear staining, while $21.4 \pm 2 \%$ of M540T/T528M cells ( $\mathrm{p}=7.4 \mathrm{E}-11)$ and $22.2 \pm$ $0.8 \%$ of $\mathrm{Y} 259 \mathrm{X} / \mathrm{Y} 259 \mathrm{X}$ cells $(\mathrm{p}=2.3 \mathrm{E}-10)$ were detected with positive nuclear staining. Hence, nuclear permeability was significantly higher in these cells than could be anticipated from basal dextran uptake, for instance due to mitotic events, as seen in control cells.

\section{Reversible loss of NLS-EYFP from laminopathy nuclei}

The number of dextran positive nuclei in laminopathy cells increased gradually with time after scrape-loading (Fig. 1b). To determine whether this increase in nuclear permeability was due to reduced pore function leading to gradual, continuous uptake or rather due to larger structural defects resulting in more abrupt leakage of the nuclear membrane, we performed detailed live-cell imaging experiments using EYFP-tagged nuclear localization signal (EYFP-NLS). We chose this marker for nuclear compartmentalization to guarantee minimal interference with basal cell physiology. Dermal fibroblast cultures from patients suffering from various laminopathies were transfected with EYFP-NLS and monitored in time using confocal time-lapse microscopy. Both short-term (2 hours at $1 \mathrm{~min}$ intervals) and long-term (24 hours at 515 min intervals) recordings were acquired. All interphase cells from two different batches of NHDF $\alpha$ control cells retained a stable intranuclear EYFP-NLS signal intensity throughout a 2-24 hour recording period (Table 1). In contrast, several laminopathy-patient fibroblasts showed a sudden decrease of EYFPNLS signal in the nucleus and concurrent increase of EYFP-NLS signal in the surrounding cytoplasm (Fig. 2-3, Table 1 and Videos 1-4). After this apparent loss of nuclear compartmentalization, restoration of EYFP-NLS signal was observed in the nucleus with recovery times varying between 5 and $60 \mathrm{~min}$. Frequently, the loss of EYFP-NLS signal initiated at sites with aberrant morphology. M540T/T528M cells typically display herniations (blebs) of the nuclear membrane. It was exactly at these aberrant regions that the nuclear membrane ruptured (Fig. 2a, Videos 1-3). However, ruptures could also be detected in nuclei without any overt morphological aberrations (Fig. 2c, Video 4). Although there is a correlation between the frequency of nuclear ruptures and aberrant morphology at the cell population level, there is no one-to-one relationship (Table 1). Of all cells with nuclear herniations 55\% showed nuclear rupture within a 120 min recording period, while $12.5 \%$ of the nuclei that were judged as 
morphologically normal also showed this phenomenon. Obviously, EYFP-NLS does not reveal more subtle abnormalities such as changes in composition of the nuclear envelope. Using EGFP-Lamin B1 in conjunction with mCherry-NLS, we found ruptures to occur at sites with reduced abundance or complete absence of Lamin B (Fig. 4). To avoid any interference with the lamina by over expression of lamins and still obtain a view on the organization of the nuclear envelope, we selectively labeled nuclear pore complexes (NPCs) using an EGFP-NUP153 construct. This demonstrated that bleb formation and ruptures also occurred at sites of the nuclear membrane with reduced abundance or complete absence of nuclear pores (Fig. 2, 4-5, Video 5). Typically, in patient cells NPC-poor regions were also devoid of Lamin B1 (Fig. 5).

\begin{tabular}{|c|c|c|c|c|}
\hline $\begin{array}{l}\text { Human } \\
\text { dermal } \\
\text { fibroblast } \\
\text { culture }\end{array}$ & Gene & Mutation & $\begin{array}{l}\text { Cells }(\% \pm \mathrm{SD}) \text { with } \\
\text { nuclear } \\
\text { abnormalities } \\
\text { (herniations and } / \text { or } \\
\text { honeycombs, } \\
\mathrm{n}=3 \times 100 \text { cells) }\end{array}$ & $\begin{array}{l}\text { EYFP-NLS expressing } \\
\text { cells (\%) showing } \\
\text { nuclear ruptures in a } 2 \mathrm{~h} \\
\text { recording period }\left(\mathrm{n}^{\circ} \text { of }\right. \\
\text { rupturing cells } / \text { total } \mathrm{n}^{\circ} \\
\text { of cells) }\end{array}$ \\
\hline NHDF $\alpha-1$ & $L M N A$ & $+/+$ & $5.7 \pm 0.6$ & $0(0 / 23)$ \\
\hline NHDF $\alpha-2$ & LMNA & $+/+$ & $2.3 \pm 1.5$ & $0(0 / 149)$ \\
\hline Double-null & $L M N A$ & Y259X/Y259X & $63.0 \pm 5.0(p=3 \mathrm{E}-05)^{*}$ & $49.5(93 / 188)$ \\
\hline Progeroid & LMNA & M540T/T528M & $38.8 \pm 9.0(\mathrm{p}=7 \mathrm{E}-05)^{*}$ & $29.1(39 / 134)$ \\
\hline $\begin{array}{l}\text { Father } \\
\text { Progeroid }\end{array}$ & LMNA & $+/ \mathrm{T} 528 \mathrm{M}$ & $13.5 \pm 11.6(p=0.29)$ & $14.3(3 / 21)$ \\
\hline $\begin{array}{l}\text { Mother } \\
\text { Progeroid }\end{array}$ & LMNA & $+/ \mathrm{M} 540 \mathrm{~T}$ & $9 \pm 4.6(\mathrm{p}=0.31)$ & $0(0 / 23)$ \\
\hline HGPS & LMNA & +/G608G & $13.0 \pm 2.3(p=7 E-04)^{*}$ & $13.6(21 / 154)$ \\
\hline FPLD & $L M N A$ & $+/ \mathrm{R} 439 \mathrm{C}$ & $16.5 \pm 3.5(p=3 E-03)^{*}$ & $24.6(15 / 61)$ \\
\hline $\mathrm{RD}$ & ZMPSTE & $\begin{array}{l}\text { c.1085- } \\
\text { 1086insT/c.1085- } \\
\text { 1086insT }\end{array}$ & $\begin{array}{l}27.57 \quad \pm 3.2 \quad(p=8 E- \\
05)^{*}\end{array}$ & $5.2(3 / 58)$ \\
\hline $\mathrm{HCM}$ & $L M N A$ & $+/ \mathrm{R} 644 \mathrm{C}$ & $5.0 \pm 1.7(p=0.56)$ & $7.7(7 / 91)$ \\
\hline
\end{tabular}

* Significantly different from control NHDF $\alpha-1$ fibroblast culture as determined from a Students' ttest (p-value $<0.05)$

Table 1. Comparison of nuclear abnormalities, NLS-EYFP visualized nuclear ruptures and occurrence of cytoplasmic PML bodies in different laminopathy fibroblast cultures. 

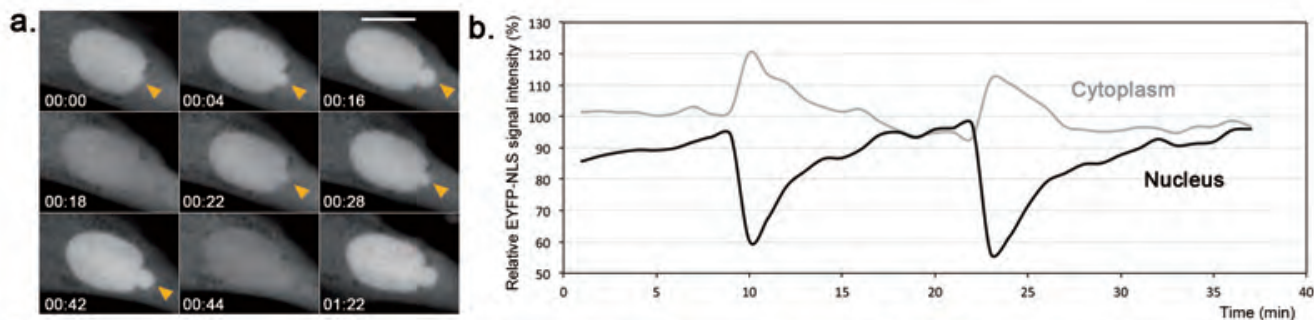

c.

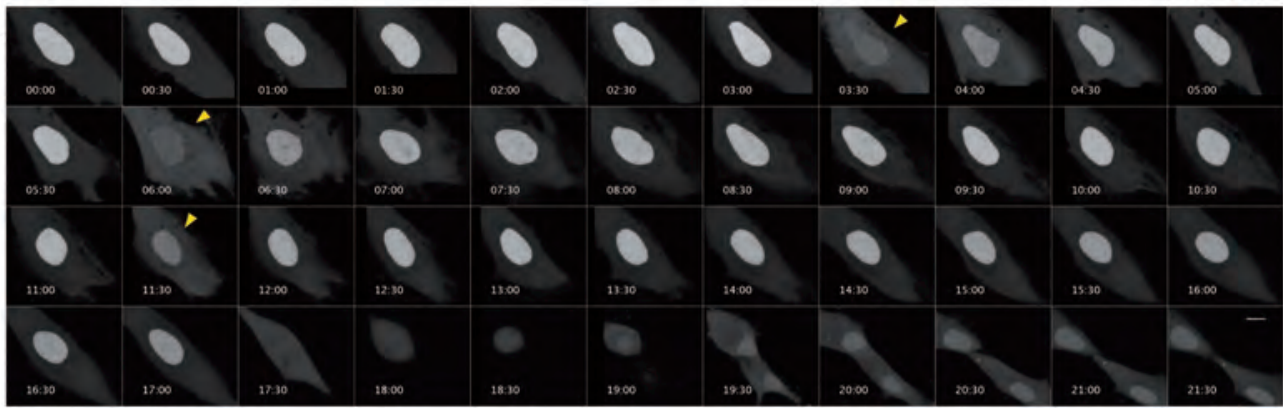

d.

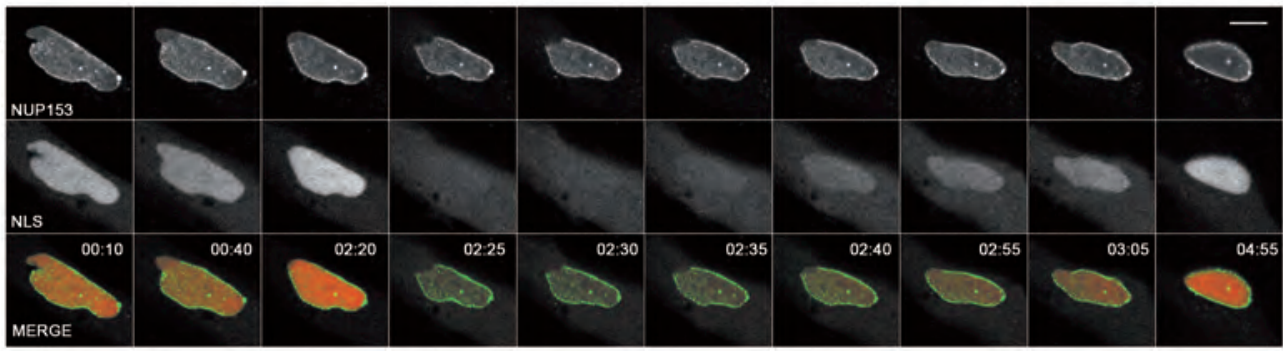

Figure 2. Non-lethal nuclear ruptures. (a) Selected images from a time lapse recording of an M540T/T528M fibroblast transfected with EYFP-tagged nuclear localization signal (EYFP-NLS), sampled at 2 minute intervals for 2 hours (Video 1). Note the nuclear herniation (arrowhead) that forms the initiation site for rupture: the herniation gradually grows until it bursts, accompanied by a direct decrease in intranuclear EYFP signal and increase of cytoplasmic EYFP signal. Subsequently, a gradual recovery of the nuclear signal can be observed along with a reformation of the herniation. A second burst is again followed by a recovery of the nuclear signal indicating restoration of the nuclear membrane integrity. (b) Temporal quantification of nuclear and cytoplasmic EYFP-NLS signal (mean intensity) of the cell shown in (a). (c) Montage of a confocal time lapse recording of a Y259X/Y259X cell 24 hours after transfection with EYFPNLS, sampled at 30 min intervals (see also Video 3). Confocal image stacks have been maximum intensity projected and rigidly registered (correlation based translation and rotation) for display purposes. The cell undergoes three consecutive rupture events (indicated by orange arrowheads), visible by the simultaneous decrease of nuclear signal and increase of cytoplasmic signal, each time followed by a restoration of the nuclear signal. After approximately $17 \mathrm{~h}$ of monitoring, the cell goes through mitosis. (d) Montage of a confocal time lapse recording of a Y259X/Y259X cell 24 hours after double transfection with EGFPNUP153 and NLS-mCherry, sampled at 5 min intervals, showing a rupture initiated at a site with reduced abundance of nuclear pore complexes (top left, Video 5). The scale bars represent $10 \mu \mathrm{m}$. 

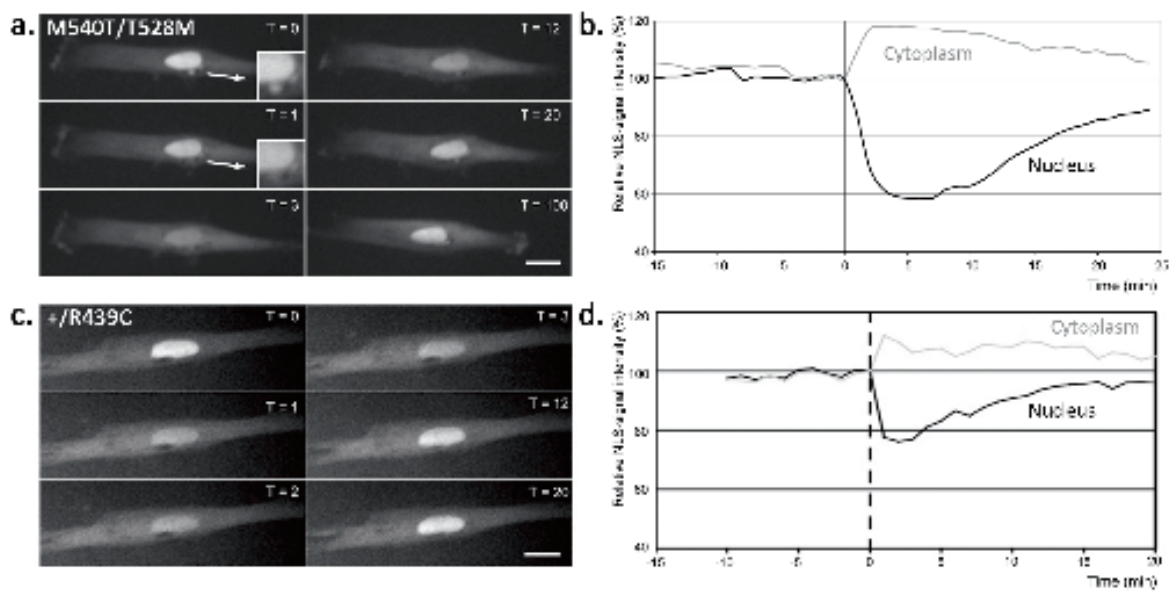

Figure 3. Dynamic ruptures of the nuclear membrane in M540T/T528M (a,b) and +/R439C cells (c,d). (a) Selected images from a time-lapse recording of a M540T/T528M fibroblast transfected with EYFP-tagged nuclear localization signal (EYFP-NLS), sampled at 1 minute intervals for 2 hours (for the complete recording see Supplementary Material, Video S2). Time point $0(\mathrm{t}=15 \mathrm{~min}$ in Video S2) is the image just before rupture, time point 1 is the image just after rupture. Note the nuclear herniation (inset) that forms the initiation site for rupture: the signal decrease starts at the herniation and is followed by a simultaneous decrease in intranuclear EYFP signal and increase of cytoplasmic EYFP signal. Subsequently, a gradual recovery of the nuclear signal can be observed, indicating restoration of the nuclear membrane integrity; (c) Selected images from a time-lapse recording of a LMNA ${ }^{\mathrm{R} 439 \mathrm{C} /+}$ (FPLD) fibroblast transfected with EYFPNLS showing rupture (b,d) Temporal quantification of nuclear and cytoplasmic EYFP-NLS signal (mean intensity) of the cell shown in a and c respectively. Scale bars represents $10 \mu \mathrm{m}$.

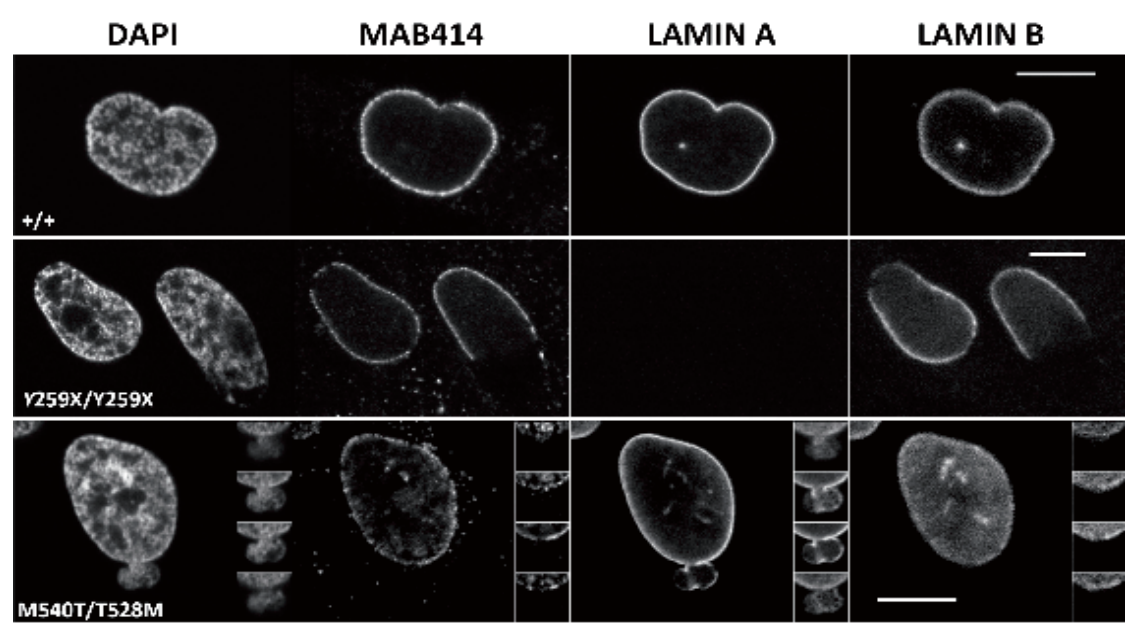

Figure 4. Representative images of nuclei from +/+ cells, Y259X/Y259X cells and M540T/T528M cells after immunofluorescence staining for a general nuclear pore marker using antibody MAB414, Lamin A and Lamin B and counterstaining with DAPI. Note the local absence of both nuclear pores and Lamin B in Y259X/Y259X cells and M540T/T528M cells. The inset in the montage of the M540T/T528M nucleus shows herniation at different focal planes to illustrate the honeycomb labeling pattern of Lamin A. Scale bars represent $10 \mu \mathrm{m}$. 

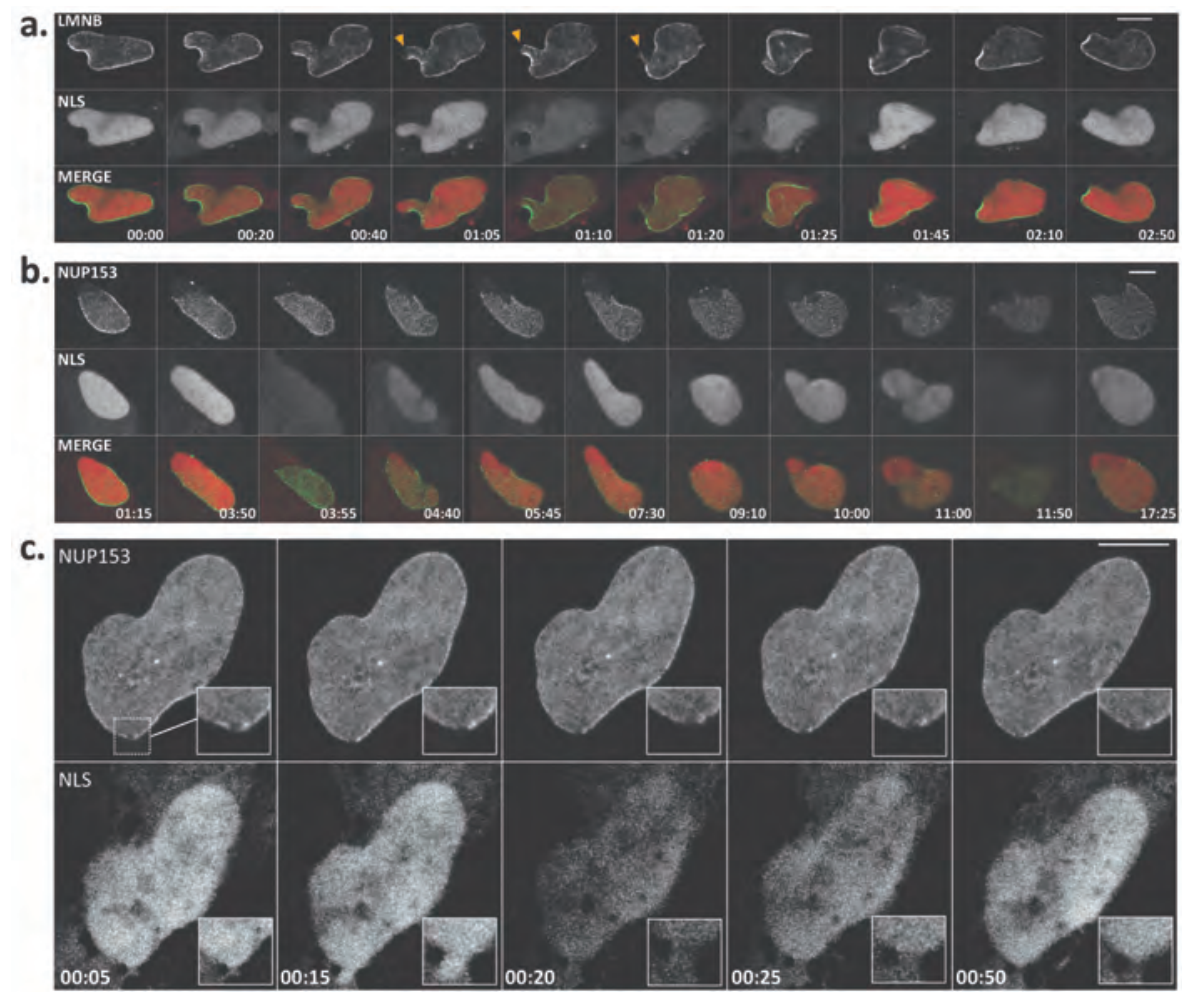

Figure 5. Ruptures occur at weak spots of the nucleus. $(\mathrm{a}, \mathrm{b})$ Montage of selected time points of a confocal time lapse recording of a Y259X/Y259X cell 24 hours after cotransfection with mCherry-NLS and EGFPLMNB (a) or mCherry-NLS and EGFP-NUP153 (b), sampled at 5min intervals. (a) During nuclear remodelling, regions form that are devoid of EGFP-LMNB signal (arrowheads), which is followed by a rupture event at time point $01: 10$ and gradual recovery; (b) Y259X/Y259X cell in which there is a permanent reduction of nuclear pores at a site of the nucleus (top left) showing recurrent rupture-recovery events at time points 03:55 and 11:50; (c) Montage of a M540T/T528M cell 24h after cotransfection with mCherry-NLS and EGFP-NUP153. The inset, a magnified view of the rectangular selection in the first time frame, shows a region, which is devoid of NUP153 signal. In this region, a bleb forms which initiates a rupture event (visualized by the mCherry-NLS signal). Confocal image stacks have been maximum intensity projected and rigidly registered for display purposes. Scale bars represent $10 \mu \mathrm{m}$.

\section{Nuclear ruptures are a direct consequence of Lamin A/C abnormalities}

In general, nuclear rupture was most frequently detected in laminopathy cells with the most severe phenotype (Table 1). For instance, cells devoid of any functional Lamin $\mathrm{A} / \mathrm{C}$ showed rupture events in almost $50 \%$ of all cells $(49.5 \%)$. A direct correlation between the absence of normal, functional A-type lamins and the frequency of ruptures 
was found by comparing the cells from a M540T/T528M patient to cells from the patients' parents. While $29.1 \%$ of the patient cells showed ruptures, fibroblasts from the father, who carries the p.T528M mutation, displayed less ruptures $(14.3 \%$, $+/ \mathrm{T} 528 \mathrm{M}$ ) and cells from the mother, carrying the p.M540T mutation, did not show any ruptures at all (+/M540T).

To confirm that reduction of functional Lamin $\mathrm{A} / \mathrm{C}$ proteins was a causal factor for nuclear membrane rupture, a stable 3T3 Lamin A/C knockdown fibroblast cell line [17] and embryonic fibroblasts (MEF) from Lamin A/C knockout mice (Lmna-/-) were transfected with EYFP-NLS and compared with their respective wild type counterparts. $18.5 \%(5 / 27)$ of the 3 T3 knockdown cells and up to $48 \%(11 / 23)$ the Lmna-/- MEFs showed ruptures in a 120 min recording period, whereas none were observed in wild type $3 \mathrm{~T} 3$ cells $(0 / 40)$ or wild type MEFs $(0 / 21)$.

Strikingly, the rupture phenomenon was also observed, albeit to a lesser extent, in laminopathy cells associated with the accumulation of unprocessed, or partly processed mutant prelamin A. $13.6 \%$ of the EYFP-NLS positive cells from a Hutchinson-Gilford Progeria Syndrome (HGPS, +/G608G) patient showed ruptures, while 5.2\% of the cells from a patient suffering from a restrictive dermopathy (RD), with homozygous c.1085_1086insT truncating mutations in the ZMPSTE24 gene, ruptured in a 2 hour recording period. The occurrence of nuclear ruptures in aforementioned cells, made us wonder whether the phenomenon was not only caused by a reduction in mature Lamin A, but could also be invoked by the accumulation of prelamin A intermediates, such as progerin. Indeed, introduction of EGFP-D50-Lamin A in normal NHDF cells resulted in nuclear rupture events in $19.6 \%$ of all recordings (11/56), suggesting the involvement of multiple causative factors.

\section{Repetitive nuclear rupture does not impede cell division}

Upon prolonged imaging of the most severely affected cell lines, i.e. Y259X/Y259X and M540T/T528M, we observed several intermittent rupture events within the same cell occurring at intervals ranging from $5 \mathrm{~min}$ up to several hours (Fig. 2). Even after multiple nuclear ruptures we observed successful cell division in these long-term recordings (Videos 3-4). The duration of mitosis in cells that ruptured was 30-60 min, similar to that in normal NHDF $\alpha$ fibroblasts.

\section{Nuclear rupture is accompanied by transient, bidirectional translocation of regulatory proteins}

To test whether the observed rupture events affected nucleocytoplasmic 
compartmentalization of essential regulatory proteins, we visualized transcription factors that should normally be restricted to the cytoplasm or the nucleus and are specifically involved with cellular stress response. As a cytoplasmic marker we used RelA, a component of the dimeric NF-kB complex, which remains sequestered in the cytoplasm in the absence of stress stimuli [32]. RelA was found to enter the nucleus of double-mutated Y259X/Y259X cells as well as Lmna-/- MEFs (Fig. 6). Simultaneous exit of mCherry-NLS confirmed that this was due to nuclear rupture and not to a sudden stress or stimulus event.

Cyclin B1, a pivotal cell-cycle regulator normally restricted to the cytoplasm of interphase cells [24], also entered the nucleus during rupture (Fig. 7), confirming that nuclear ruptures are accompanied by an influx of various cytoplasmic components. To study the behaviour of intranuclear components, we transfected Y259X/Y259X cells with a GFP-fusion construct expressing octamer transcription factor 1 (OCT1), which has been shown to reside in an insoluble nuclear fraction [33], and is partly sequestered at the nuclear periphery [20]. Indeed, we found that nuclear ruptures caused part of the OCT1 to translocate to the cytoplasm. Efflux of OCT1 was far more subtle than for EYFP-NLS and appeared to be limited to focal or diffuse regions in the nucleus (Fig. 8a, 9 and Videos 6-7).

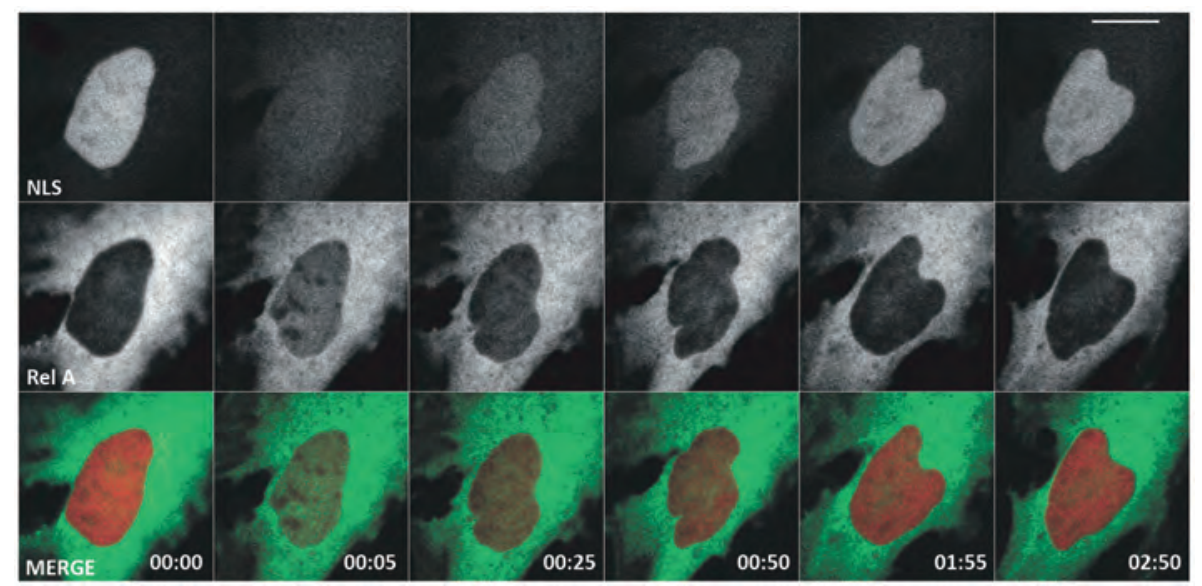

Figure 6. Nuclear ruptures lead to influx of cytoplasmic components. Montage of a confocal time lapse recording of a MEF Lmna $a^{-/}$cell 24 hours after double transfection with EGFP-RelA and mCherry-NLS, demonstrating transient influx of RelA during nuclear rupture (at time point 00:05). Scale bar represents $10 \mu \mathrm{m}$. 


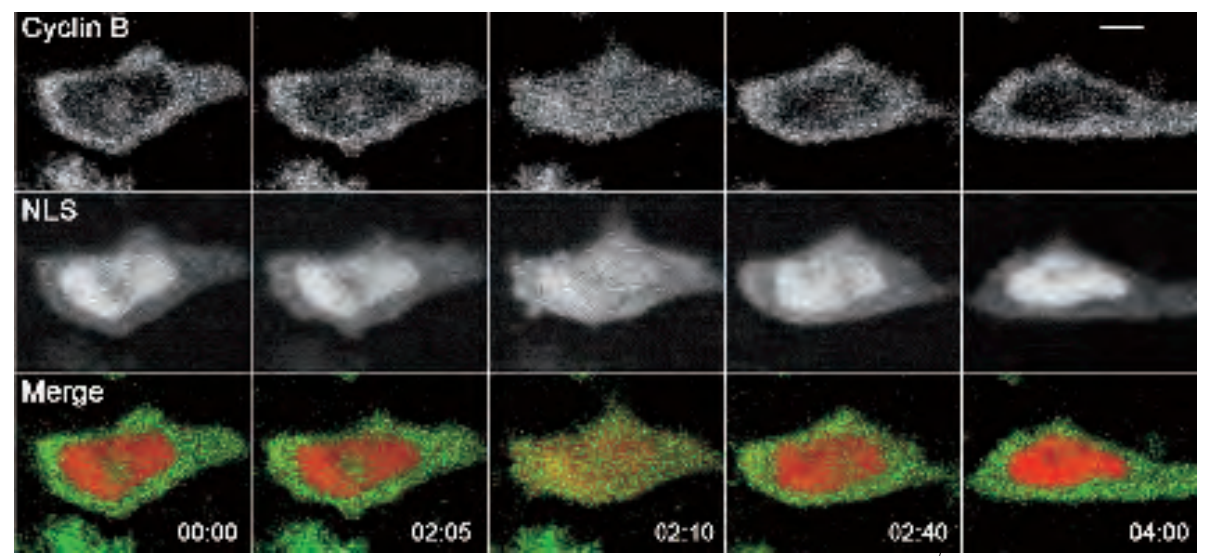

Figure 7. Montage of a confocal time lapse recording of a MEF Lmna ${ }^{-1-}$ cell 24 hours after double transfection with Cyclin B1-GFP and mCherry-NLS, demonstrating transient influx of Cyclin B1 during nuclear rupture (at time point 02:10). Scale bar represents $10 \mu \mathrm{m}$.

\section{Impaired compartmentalization may affect downstream gene expression}

We hypothesized that this impairment in spatiotemporal nucleocytoplasmic compartmentalization in laminopathy cells could have consequences for cellular function. For example, OCT1 regulates genes that are essential for the cellular stress response [34], which could mean that failure to compartmentalize OCT1 correctly causes dysregulation of these genes. As the downstream genes have been characterized in the mouse and the pathological cells of human origin could be biased by different genetic backgrounds, we determined the expression levels of Oct1-responsive genes in Lmna-/- MEFs with respect to their wild type controls and compared these with published values for Oct1-/- mouse cells [34]. Out of nine genes that were investigated, the expression of six genes (Serping1, Rdm1, Mmp13, IL6, Gpx3 and Gas5) was significantly altered in the same direction when comparing the Lmna-/- and the Oct1-/cells (Fig. 8b). 

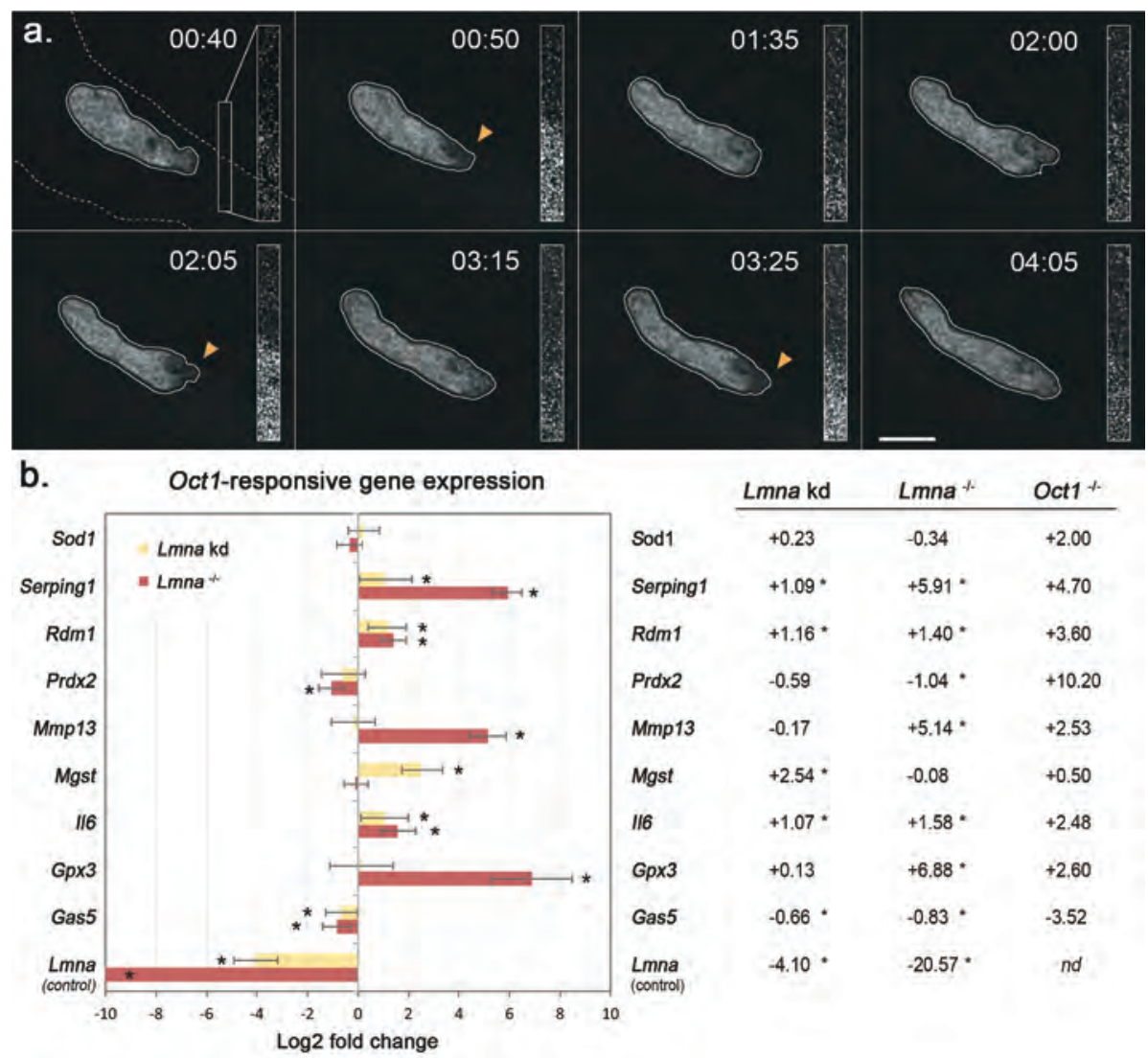

Figure 8. Nuclear rupture leads to efflux of transcription factor OCT1 and dysregulation of Oct1-responisve genes. (a) Maximum intensity projections of a confocal time lapse recording acquired at $5 \mathrm{~min}$ intervals of a Y259X/Y259X patient cell 24 hours after transfection with GFP-OCT1, showing temporary and localized loss of nuclear GFP-OCT1 during nuclear rupture at different time points (indicated by orange arrowheads, see also Supplementary Material Video S6). Contours were drawn to mark the nuclear boundary, the dotted line in the first frame demarcates the cell boundary and the inset shows a contrast-stretched, magnified view of the rectangular selection indicated in the first frame, to demonstrate the subtle increase in cytoplasmic GFP-OCT1 signal during rupture. (b) Fold changes in Oct1-responsive gene expression as measured by comparative real-time PCR in $L m n a^{-/-}$MEFs with respect to their wild type controls and compared to values described for an $\mathrm{Oct}^{-1 /} \mathrm{MEF}$ cell line (17).. Error bars represent $95 \%$ confidence intervals and log fold changes with $\mathrm{p}<0.05$ are indicated by an asterisk. 


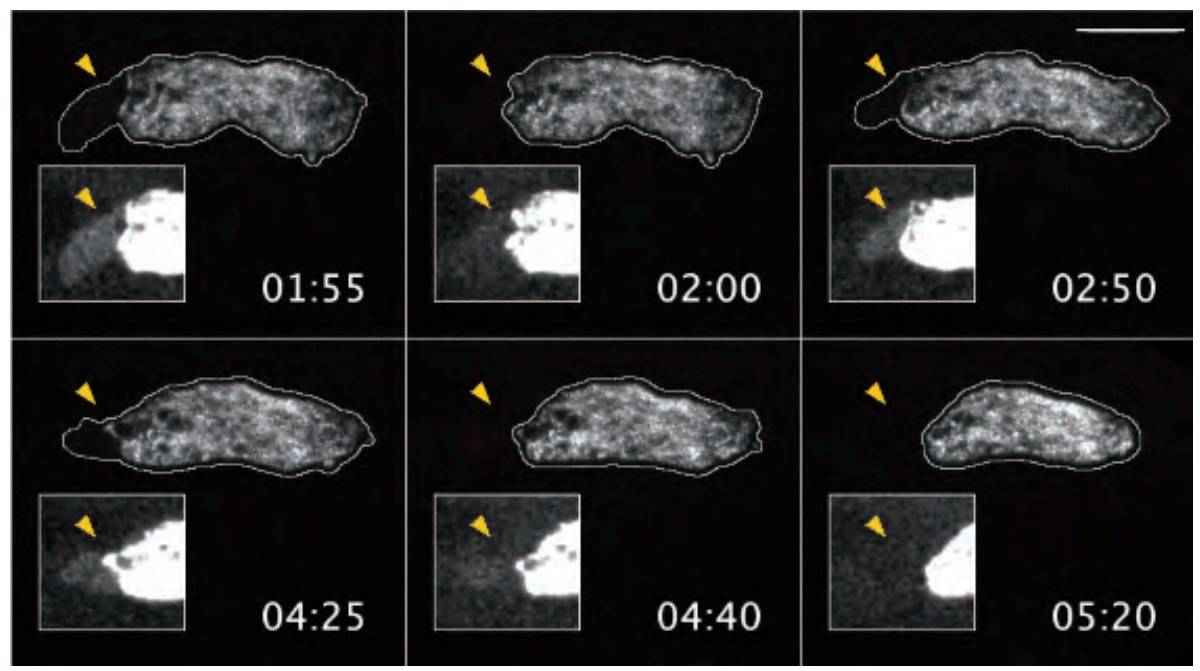

Figure 9. Ruptures induce temporary, focal reduction of OCT1. Maximum intensity projections of a confocal time-lapse recording of a Y259X/Y259X patient cell 24 hours after transfection with GFP-OCT1, showing temporary loss of diffuse nuclear GFP-OCT1 during nuclear rupture. The inset shows a contraststretched version of the nuclear herniation to visualize low intensity GFP-OCT1 signal. The herniation ruptures at $02 \mathrm{~h} 00 \mathrm{~min}$ and $04 \mathrm{~h} 40 \mathrm{~min}$. Scale bar represents $10 \mu \mathrm{m}$.

\section{Nuclear rupture is accompanied by permanent translocation of macromolecular complexes}

Triggered by the observations of uncoordinated translocation of regulatory proteins, we wondered whether larger gene-regulatory protein complexes could become relocalized during rupture as well. EYFP-tagged PML proteins were selected as markers, as these assemble into multi-protein complexes termed PML nuclear bodies, which have an important role in transcription control and DNA repair and are in general confined to the nuclear compartment [35]. Indeed, upon nuclear rupture (indicated by efflux of cotransfected NLS-EYFP), part of the EYFP-tagged PML bodies translocated from the nucleus to the cytoplasm (Fig. 10a, Video 8). As the nuclear membrane integrity recovered (indicated by restoration of nuclear EYFP-NLS signal) translocated PML structures remained excluded from the apparently restored nucleus. Some PML structures that remained in the cytoplasm gradually fragmented into smaller structures that sometimes even dissolved completely. The number of PML structures that translocated during an individual rupture ranged between 0 and 5 , but due to the repetitive nature of this event as much as $24 \%$ of the original number of nuclear PML structures were found to move out of the nucleus in long-term recordings (Video 8). 

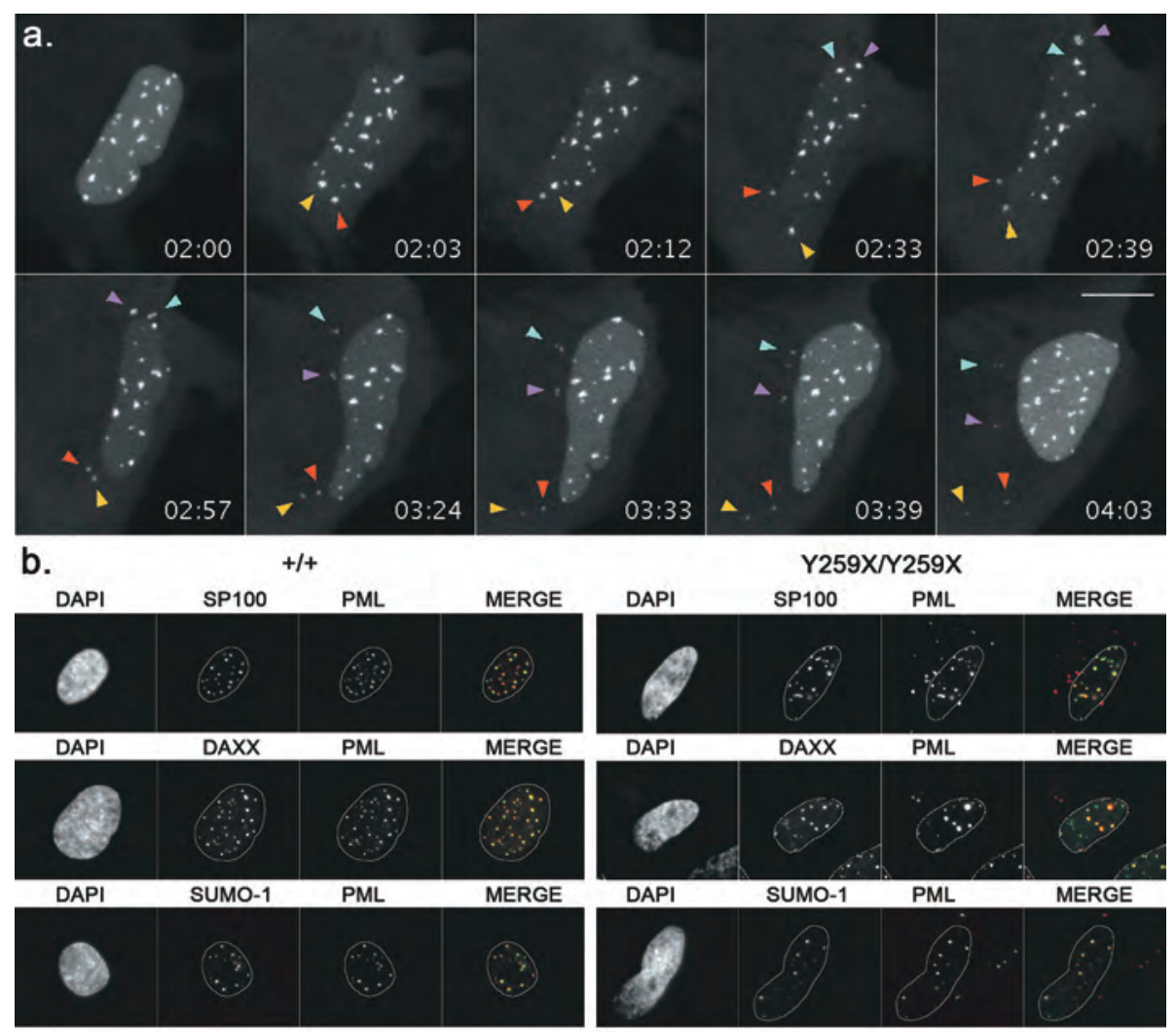

Figure 10. Nuclear rupture leads to translocation of mobile PML structures. (a) Montage of selected time points of a confocal time lapse recording of a Y259X/Y259X cell 24 hours after cotransfection with EYFPNLS and EYFP-PML, sampled at $3 \mathrm{~min}$ intervals. Confocal image stacks have been maximum intensity projected and rigidly registered (correlation based translation and rotation) for display purposes. At time point $02 \mathrm{~h} 03 \mathrm{~min}$ a nuclear rupture event takes place (evident by the sudden drop in diffuse nuclear signal) leading to the immediate translocation of two PML nuclear bodies (bottom, yellow and red arrowhead) and again escape of two PML nuclear bodies $30 \mathrm{~min}$ later at time point $02 \mathrm{~h} 33 \mathrm{~min}$ (top, cyan and magenta arrowhead). While the nuclear signal gradually restores, the cytoplasmic PML bodies translocate further away from their initial escape sites and fragment into smaller particles (cfr. magenta and cyan arrowhead at time point $03 \mathrm{~h} 33 \mathrm{~min}$ ). (b) Immunofluorescence staining of $+/+$ and $\mathrm{Y} 259 \mathrm{X} / \mathrm{Y} 259 \mathrm{X}$ cells shows that cytoplasmic PML structures are present in the laminopathy cells and do not contain typical PML body components such as DAXX, SUMO or SP100. Scale bars represent $10 \mu \mathrm{m}$.

These findings were confirmed by immunocytochemical staining of endogenous PML bodies and high-content cytometry, thereby excluding any artefacts that could arise from transfection. Only $3.2 \pm 1.3 \%$ of NHDF $\alpha$ control cells $(n=300)$ demonstrated cytoplasmic PML body staining, while $59.6 \pm 2.1 \%$ of Y259X/Y259X cells $(n=300)$ showed the presence of intensely staining PML bodies in the cytoplasm (Fig. 10b). All 
other patient cell lines examined also demonstrated a higher number of cells with cytoplasmic PML bodies, ranging from 5-18\% (data not shown). Interestingly, cytoplasmic PML structures never contained other typical PML body components such as DAXX or SP100 and were often also devoid of SUMO1. This was demonstrated by immunostaining of non-transfected cells (Fig. 10b) as well as immunolocalization after live imaging of PML structure translocation upon rupture (Fig. 11).

Similarly, translocation of large components from the cytoplasmic side to the nucleus could be indicated using electron microscopy of Y259X/Y259X cells. Prominent inclusions of non-nuclear components were found in the nucleus. These inclusions fully resemble sub cellular structures normally present in the cytoplasm (Fig. 12), such as microfilaments, vesicles, mitochondria and myeloid bodies. The inclusions are not surrounded by an additional nuclear membrane, indicating that they are not the result of nuclear membrane folding or intranuclear tubular invaginations. 
a.

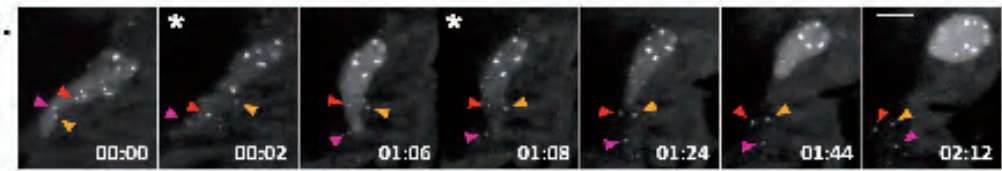

b.
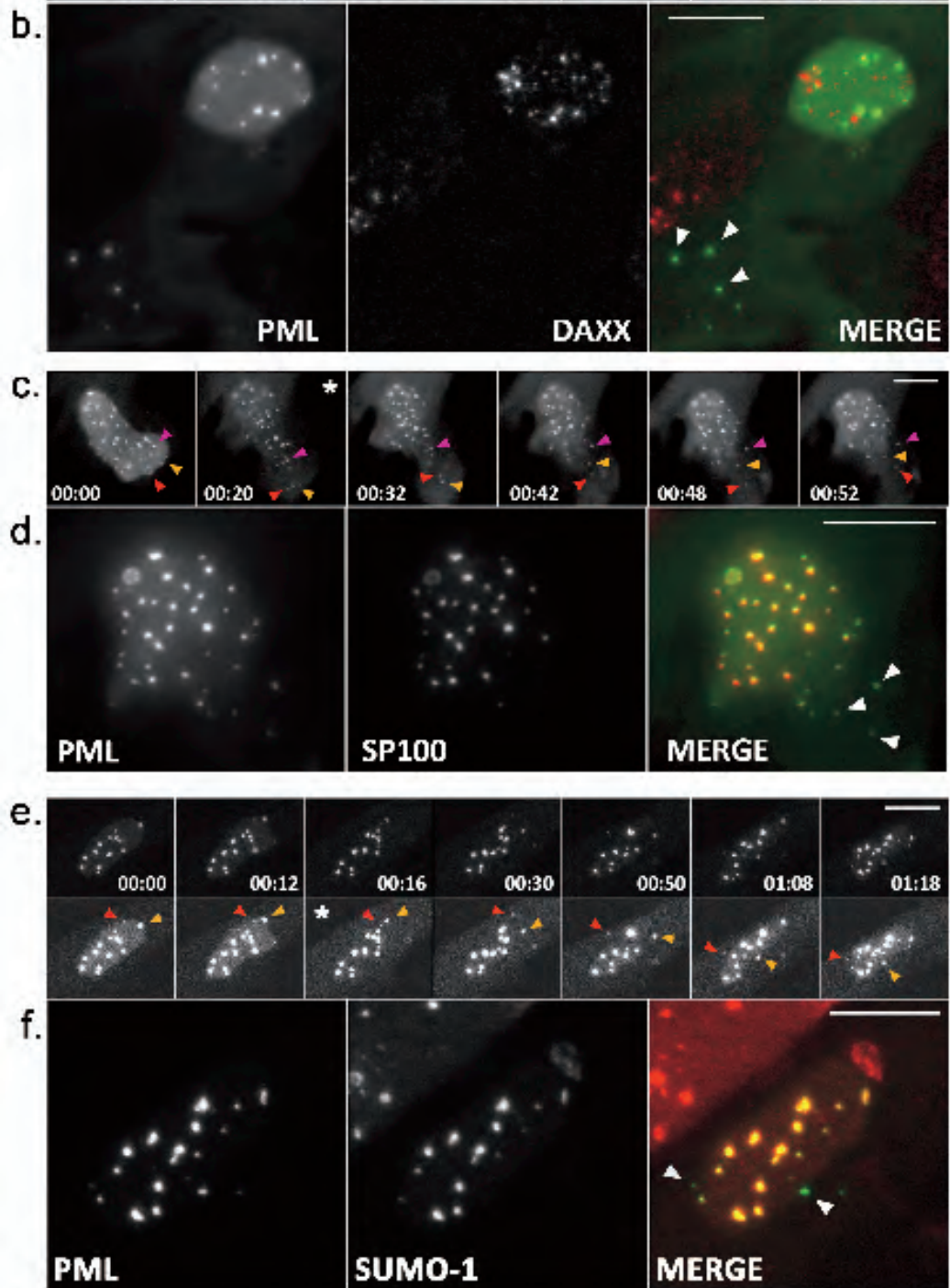

Figure 11. Immunofluorescence staining after live cell imaging of Y259X/Y259X cells cotransfected with EYFP-PML and EYFP-NLS. (a,c,e) Selected nuclei were monitored in time to detect rupture events (indicated by a $*$ ), which were associated with the translocation of nuclear PML bodies to the cytoplasm (arrowheads), after which they were fixed and immunostained for other characteristic PML body components DAXX (b), SP100 (d) and SUMO-1. This demonstrated that there was no colocalisation between the cytoplasmic PML structures and any of the other components. The montage in e is also shown as a contrast stretched version to show the weaker intensity of smaller PML foci and pan-nuclear EYFPNLS signal. Scale bars represent $10 \mu \mathrm{m}$. 

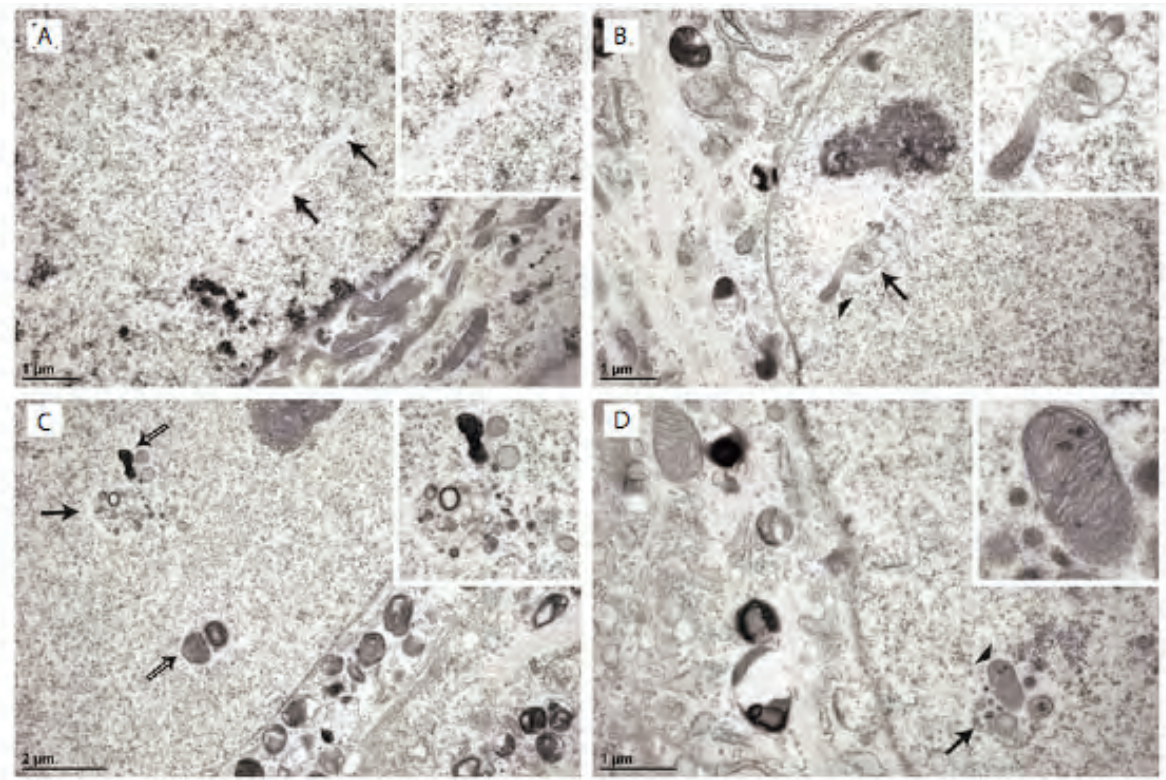

Figure 12. Electron micrographs of $\mathrm{Y} 259 \mathrm{X} / \mathrm{Y} 259 \mathrm{X}$ cells showing the presence of different cytoplasmic particles occurring inside the nucleus, including microfilaments (a, arrows), vesicles (b, c, d, arrows), mitochondria (b,d, arrowheads) and myeloid bodies (c, open arrows). Note the absence of a double lipid membrane around these structures, excluding nuclear membrane folding or intranuclear tubular invaginations to contain these structures. 


\section{Discussion}

It is well established that defective production or aberrant post-translational processing of A-type lamins elicits a broad spectrum of laminopathies that include tissue-specific (adipose, muscle, peripheral nerve) as well as systemic disorders. Considering the large variety of disease manifestations, several non-exclusive hypotheses have been proposed to relate the pathophysiology of laminopathies to lamin functions [2]. These are either based on the involvement of lamins in maintaining mechanical integrity of the nucleus (structural hypothesis), their role in modulating transcription and signalling pathways by serving as docking sites for regulatory proteins (gene expression hypothesis), or the toxicity of accumulated pre-lamin A isoforms (toxicity hypothesis). However, current hypotheses put less focus on the primary function of the nuclear envelope in basal cell physiology, namely the maintenance of a highly selective barrier between nucleus and cytoplasm.

Yet, several lines of evidence impute an important role to distorted nucleo-cytoplasmic transport in cellular degeneration. For instance, nuclear import is reduced in cell lines over expressing mutant lamin A intermediates [11], as well as in cells undergoing senescence [36]. In addition, in aging cells, impaired nuclear pore function has been observed, leading to increased nuclear permeability and the leakage of cytoplasmic proteins, such as tubulin, into the nucleus [37]. The link between the influx of cytoplasmic tubulin into the nucleus with the aging process, as well as neurodegenerative diseases [38] emphasises the role of (deregulated) nuclear transport in development of human pathologies. Our findings on the occurrence of nuclear ruptures in cells from different laminopathy patients clearly support this connection, but also add a novel aspect to the suggested paradigm: instead of a gradual, chronic transport problem, suggested to be caused by decreased levels of import factors [36] or the presence of oxidatively damaged pore complexes [37], we now document sudden disruptions of the nuclear compartment which lead to an immediate intermingling of nuclear and cytoplasmic components. Although the exact nature of these ruptures is not yet known, there appears to be a correlation between the reduction of mature, nonmutant lamin A/C proteins and the frequency of nuclear ruptures. Different lines of evidence point to this correlation.

First, both Lmna knockdown in 3T3 cells and Lmna knockout in mouse embryonic fibroblasts result in nuclear ruptures, which were never observed in wild-type cells.

Secondly, the progeroid patient cells (M540T/T528M), expressing only mutant A-type lamins show a much higher number of nuclear ruptures, as compared to the cells from 
the heterozygous parents, each bearing one normal LMNA allele. While lamin $\mathrm{A} / \mathrm{C}$ deficiency has been shown to result in increased nuclear fragility and apoptosis in cells exposed to mechanical stress [39], the ruptures described here occur under basal culturing conditions without application of external force, are transient in nature and are non-lethal. Ruptures often initiated at weak spots of the nucleus, typified by reduced presence or even complete absence of other nuclear envelope components such as lamin B or nuclear pore complexes, indicating a structural basis for this phenomenon. Indeed, structural alterations in the lamina either weaken or stiffen the nuclear envelope, in case of a lamin A/C deficiency [40] or progerin accumulation [41], respectively. Both conditions may render the nucleus more susceptible to local damage. In HGPS cells a reduced compliance of rigid nuclei to pulling forces, exerted by the cytoskeleton, could cause local fractures in the nuclear envelope [41]. Alternatively, in lamin A deficient cells, a defective connection with the cytoskeleton [12] augments nuclear mobility and deformation [40], thereby possibly increasing the risk of ruptures, especially at fragile sites of the imperfect nuclear envelope.

The exact impact of dynamic nuclear ruptures on cell behaviour, differentiation and cellular lifespan remains elusive, but it is conceivable that the abrupt loss of compartmentalization affects cellular functioning in several ways. For instance, the sudden mislocalization of essential transcription factors upon nuclear rupture may alter gene expression. Previous studies have indicated that lamins form a scaffold for tethering several signalling proteins and transcription factors (ERK, pRb, SREBP1, OCT-1 etc.) and thereby modulate their activity $[42,43]$. As we show here, LMNA mutations will not only alter these interactions directly but also invoke unintentional, bidirectional diffusion of various transcription factors or signalling proteins; a phenomenon, which may be missed by classical biochemical approaches. The altered stoichiometry of resident nuclear proteins is prone to shift DNA-binding kinetics and thereby indirectly affect gene regulation permanently or temporarily ([44-46]. This is supported by our data on Oct1-responsive gene expression, which indeed becomes altered in Lmna -/- cells in a similar way as in Oct1-/- cells and in an opposite sense with respect to $L m n b^{\Delta / \Delta}$ cells [20], plausibly due to reduced abundance of nucleoplasmic Oct1. The fact that not all of the investigated $O c t 1$-responsive genes react similarly to the (partial) loss of Lmna transcripts may be explained by the fact that ruptures invoke translocation of a variety of transcription factors (e.g. RelA) and thus influence gene expression in a highly complex manner. Moreover, A-type lamins influence gene expression in a multifactor fashion, for instance by sequestration of transcription factors at the periphery and by their role in chromatin organization [42, 43]. The alterations in $O c t 1$-responsive gene expression may cause cells failing to act timely and adequately to oxidative stress. As such, it raises the intriguing possibility 
that nuclear ruptures connect structural and gene regulatory aspects of disease development.

Alternatively, mislocalization of cellular proteins could impose an extra load on the cell for recuperating material to the correct compartment by means of nucleocytoplasmic transport, a process that may become especially strenuous in a context of aging or prelamin A accumulation, i.e. conditions where reduced normal nuclear import is observed [11, 36, 37, 47].

Accumulation of translocated material in the wrong cellular compartment may be targeted for degradation by the proteasomes, but large amounts of mislocalized protein could increase the risk of proteasome saturation and consequent aggresome formation or even induction of apoptosis [47, 48].

Next to transient shuttling of regulatory proteins, ruptures were also accompanied by the translocation of macromolecular complexes such as PML containing structures. These mobile PML structures were devoid of typical PML body components DAXX and SP-100 and often also SUMO1. In composition they resemble PML microstructures, which are produced after heavy metal or heat-shock treatment [49], but unlike these PML microstructures, the satellite PML structures we observed can be as large as classical nuclear PML bodies. Two possible translocation mechanisms can be envisaged: either satellite PML structures exist in the nucleus that lack interactions with chromatin rendering them more mobile and allowing them to escape the nucleus upon rupture; or alternatively, translocated PML bodies rapidly lose their other components quickly, for instance due to desumoylation (cfr. mitotic PML accumulations [50]), leading to gradual defragmentation. Although it is tempting to speculate on a role in stress response, analogous to PML microstructures [49], the exact function of these satellite PML structures remains to be resolved.

Dynamic ruptures of nuclear envelope herniations have previously been observed in HIV infected cells [51]. It is now known that HIV and also other virus infections weaken the nuclear lamina, potentially to facilitate viral entry into the nucleus, confirming the critical role for lamins in maintaining subcellular compartmentalization. Moreover, strong indications exist that nuclear rupture is not confined to in vitro cell systems. Electron microscopic studies show local absence of nuclear membranes in cardiomyocytes of heart biopsies [9, 10], as well as in striated muscle cells [52] of laminopathy patients. As various other pathologic cell cultures such as small lung carcinoma cells also display a dramatic decrease in lamin $\mathrm{A} / \mathrm{C}$ expression [53], as well as undifferentiated cell who barely express lamin A/C [54-59], we speculate that temporary decompartmentalization may have a more generic relevance for development of diseases associated with an altered Lamin A/C metabolism. 


\section{Acknowledgements}

We acknowledge the invaluable contributions made by the families consenting to donate skin biopsies for fibroblast cultures. Dr. R. Hennekam (University of Amsterdam, the Netherlands) and Prof. Dr. B. Van Engelen (UMC St Radboud University, the Netherlands) are acknowledged for sharing the HGPS (+/G608G) and double-null (Y259X/Y259X) patient cell cultures, respectively. Dr. B. Burke and Dr. C. Stewart (Institute of Medical Biology, Singapore) are acknowledged for providing MEF Lmna-/- cells. This work was supported by the Hercules Foundation (Belgium) and NBphotonics, Ghent University (Belgium).

\section{Video}

Video 1:

http://dl.dropbox.com/u/37827993/Chapter4-video1.avi

Video 2:

http://dl.dropbox.com/u/37827993/Chapter4-video2.avi

Video 3:

http://dl.dropbox.com/u/37827993/Chapter4-video3.avi

Video 4:

http://dl.dropbox.com/u/37827993/Chapter4-video4.avi

Video 5:

http://dl.dropbox.com/u/37827993/Chapter4-video5.avi

Video 6:

http://dl.dropbox.com/u/37827993/Chapter4-video6.avi

Video 7:

http://dl.dropbox.com/u/37827993/Chapter4-video7.avi

Video 8:

http://dl.dropbox.com/u/37827993/Chapter4-video8.avi 


\section{References}

[1] M.W. Hetzer, T.C. Walther and I.W. Mattaj, Pushing the envelope: structure, function, and dynamics of the nuclear periphery, Annu Rev Cell Dev Biol 21 (2005) 347-380.

[2] J.L. Broers, F.C. Ramaekers, G. Bonne, R.B. Yaou and C.J. Hutchison, Nuclear lamins: laminopathies and their role in premature ageing, Physiol Rev 86 (2006) 967-1008.

[3] J.L. Broers, C.J. Hutchison and F.C. Ramaekers, Laminopathies, J Pathol 204 (2004) 478-488.

[4] M. Cohen, K.K. Lee, K.L. Wilson and Y. Gruenbaum, Transcriptional repression, apoptosis, human disease and the functional evolution of the nuclear lamina, Trends Biochem Sci 26 (2001) 41-47.

[5] K.L. Wilson, M.S. Zastrow and K.K. Lee, Lamins and disease: insights into nuclear infrastructure, Cell 104 (2001) 647-650.

[6] S. Vlcek and R. Foisner, A-type lamin networks in light of laminopathic diseases, Biochim Biophys Acta 1773 (2007) 661-674.

[7] M. Zwerger, T. Kolb, K. Richter, I. Karakesisoglou and H. Herrmann, Induction of a massive endoplasmic reticulum and perinuclear space expansion by expression of lamin $\mathrm{B}$ receptor mutants and the related sterol reductases TM7SF2 and DHCR7, Mol Biol Cell 21 (2010) 354-368.

[8] R.D. Goldman, D.K. Shumaker, M.R. Erdos, M. Eriksson, A.E. Goldman, L.B. Gordon, Y. Gruenbaum, S. Khuon, M. Mendez, R. Varga and F.S. Collins, Accumulation of mutant lamin A causes progressive changes in nuclear architecture in Hutchinson-Gilford progeria syndrome, Proc Natl Acad Sci U S A 101 (2004) 8963-8968.

[9] A. Fidzianska, Z.T. Bilinska, F. Tesson, T. Wagner, M. Walski, J. Grzybowski, W. Ruzyllo and I. Hausmanowa-Petrusewicz, Obliteration of cardiomyocyte nuclear architecture in a patient with LMNA gene mutation, $\mathrm{J}$ Neurol Sci 271 (2008) 91-96.

[10] P. Gupta, Z.T. Bilinska, N. Sylvius, E. Boudreau, J.P. Veinot, S. Labib, P.M. Bolongo, A. Hamza, T. Jackson, R. Ploski, M. Walski, J. Grzybowski, E. Walczak, G. Religa, A. Fidzianska and F. Tesson, Genetic and ultrastructural studies in dilated cardiomyopathy patients: a large deletion in the lamin $\mathrm{A} / \mathrm{C}$ gene is associated with cardiomyocyte nuclear envelope disruption, Basic Res Cardiol 105 (2010) 365-377.

[11] A. Busch, T. Kiel, W.M. Heupel, M. Wehnert and S. Hubner, Nuclear protein import is reduced in cells expressing nuclear envelopathy-causing lamin A mutants, Exp Cell Res 315 (2009) 2373-2385.

[12] A. Muchir, B.G. van Engelen, M. Lammens, J.M. Mislow, E. McNally, K. Schwartz and G. Bonne, Nuclear envelope alterations in fibroblasts from 
LGMD1B patients carrying nonsense Y259X heterozygous or homozygous mutation in lamin A/C gene, Exp Cell Res 291 (2003) 352-362.

[13] V.L. Verstraeten, J.L. Broers, M.A. van Steensel, S. Zinn-Justin, F.C. Ramaekers, P.M. Steijlen, M. Kamps, H.J. Kuijpers, D. Merckx, H.J. Smeets, R.C. Hennekam, C.L. Marcelis and A. van den Wijngaard, Compound heterozygosity for mutations in LMNA causes a progeria syndrome without prelamin A accumulation, Hum Mol Genet 15 (2006) 2509-2522.

[14] D.B. Savage, M.A. Soos, A. Powlson, S. O'Rahilly, I. McFarlane, D.J. Halsall, I. Barroso, E.L. Thomas, J.D. Bell, I. Scobie, P.E. Belchetz, W.F. Kelly and A.J. Schafer, Familial partial lipodystrophy associated with compound heterozygosity for novel mutations in the LMNA gene, Diabetologia 47 (2004) 753-756.

[15] V.L. Verstraeten, S. Caputo, M.A. van Steensel, I. Duband-Goulet, S. ZinnJustin, M. Kamps, H.J. Kuijpers, C. Ostlund, H.J. Worman, J.J. Briede, C. Le Dour, C.L. Marcelis, M. van Geel, P.M. Steijlen, A. van den Wijngaard, F.C. Ramaekers and J.L. Broers, The R439C mutation in LMNA causes lamin oligomerization and susceptibility to oxidative stress, J Cell Mol Med 13 (2009) 959-971.

[16] C.L. Moulson, G. Go, J.M. Gardner, A.C. van der Wal, J.H. Smitt, J.M. van Hagen and J.H. Miner, Homozygous and compound heterozygous mutations in ZMPSTE24 cause the laminopathy restrictive dermopathy, J Invest Dermatol 125 (2005) 913-919.

[17] F. Houben, C.H. Willems, I.L. Declercq, K. Hochstenbach, M.A. Kamps, L.H. Snoeckx, F.C. Ramaekers and J.L. Broers, Disturbed nuclear orientation and cellular migration in A-type lamin deficient cells, Biochim Biophys Acta 1793 (2009) 312-324.

[18] T. Sullivan, D. Escalante-Alcalde, H. Bhatt, M. Anver, N. Bhat, K. Nagashima, C.L. Stewart and B. Burke, Loss of A-type lamin expression compromises nuclear envelope integrity leading to muscular dystrophy, J Cell Biol 147 (1999) 913-920.

[19] G.J. Kremers, J. Goedhart, E.B. van Munster and T.W. Gadella, Jr., Cyan and yellow super fluorescent proteins with improved brightness, protein folding, and FRET Forster radius, Biochemistry 45 (2006) 6570-6580.

[20] A.N. Malhas, C.F. Lee and D.J. Vaux, Lamin B1 controls oxidative stress responses via Oct-1, J Cell Biol 184 (2009) 45-55.

[21] Y.C. Lussi, D.K. Shumaker, T. Shimi and B. Fahrenkrog, The nucleoporin Nup153 affects spindle checkpoint activity due to an association with Mad1, Nucleus 1 (2010) 71-84.

[22] L. Chen, W. Fischle, E. Verdin and W.C. Greene, Duration of nuclear NFkappaB action regulated by reversible acetylation, Science 293 (2001) 16531657. 
[23] K. Wiesmeijer, C. Molenaar, I.M. Bekeer, H.J. Tanke and R.W. Dirks, Mobile foci of Sp100 do not contain PML: PML bodies are immobile but PML and Sp100 proteins are not, J Struct Biol 140 (2002) 180-188.

[24] J. Pines and T. Hunter, Human cyclins A and B1 are differentially located in the cell and undergo cell cycle-dependent nuclear transport, J Cell Biol 115 (1991) 1-17.

[25] A. Hagting, M. Jackman, K. Simpson and J. Pines, Translocation of cyclin B1 to the nucleus at prophase requires a phosphorylation-dependent nuclear import signal, Curr Biol 9 (1999) 680-689.

[26] P. Scaffidi and T. Misteli, Reversal of the cellular phenotype in the premature aging disease Hutchinson-Gilford progeria syndrome, Nat Med 11 (2005) 440445 .

[27] W.H. De Vos, R.A. Hoebe, G.H. Joss, W. Haffmans, S. Baatout, P. Van Oostveldt and E.M. Manders, Controlled light exposure microscopy reveals dynamic telomere microterritories throughout the cell cycle, Cytometry A 75 (2009) 428-439.

[28] M.D. Abramoff, P.J. Magalhaes and S.J. RAM, Image Processing with ImageJ, Biophotonics 11 (2004) 36-42.

[29] P.L. McNeil, R.F. Murphy, F. Lanni and D.L. Taylor, A method for incorporating macromolecules into adherent cells, J Cell Biol 98 (1984) 15561564.

[30] W.H. De Vos, L. Van Neste, B. Dieriks, G.H. Joss and P. Van Oostveldt, High content image cytometry in the context of subnuclear organization, Cytometry A 77 (2010) 64-75.

[31] K.J. Livak and T.D. Schmittgen, Analysis of relative gene expression data using real-time quantitative PCR and the 2(-Delta Delta C(T)) Method, Methods 25 (2001) 402-408.

[32] S. Vallabhapurapu and M. Karin, Regulation and function of NF-kappaB transcription factors in the immune system, Annu Rev Immunol 27 (2009) 693-733.

[33] M.K. Kim, L.A. Lesoon-Wood, B.D. Weintraub and J.H. Chung, A soluble transcription factor, Oct-1, is also found in the insoluble nuclear matrix and possesses silencing activity in its alanine-rich domain, Mol Cell Biol 16 (1996) 4366-4377.

[34] D. Tantin, C. Schild-Poulter, V. Wang, R.J. Hache and P.A. Sharp, The octamer binding transcription factor Oct-1 is a stress sensor, Cancer Res 65 (2005) 10750-10758.

[35] R. Bernardi and P.P. Pandolfi, Structure, dynamics and functions of promyelocytic leukaemia nuclear bodies, Nat Rev Mol Cell Biol 8 (2007) 1006-1016.

[36] G. Pujol, H. Soderqvist and A. Radu, Age-associated reduction of nuclear protein import in human fibroblasts, Biochem Biophys Res Commun 294 (2002) 354-358. 
[37] M.A. D'Angelo, M. Raices, S.H. Panowski and M.W. Hetzer, Age-dependent deterioration of nuclear pore complexes causes a loss of nuclear integrity in postmitotic cells, Cell 136 (2009) 284-295.

[38] J.M. Woulfe, Abnormalities of the nucleus and nuclear inclusions in neurodegenerative disease: a work in progress, Neuropathol Appl Neurobiol 33 (2007) 2-42.

[39] J. Lammerding, P.C. Schulze, T. Takahashi, S. Kozlov, T. Sullivan, R.D. Kamm, C.L. Stewart and R.T. Lee, Lamin A/C deficiency causes defective nuclear mechanics and mechanotransduction, J Clin Invest 113 (2004) 370378.

[40] W.H. De Vos, F. Houben, R.A. Hoebe, R. Hennekam, B. van Engelen, E.M. Manders, F.C. Ramaekers, J.L. Broers and P. Van Oostveldt, Increased plasticity of the nuclear envelope and hypermobility of telomeres due to the loss of A-type lamins, Biochim Biophys Acta 1800 (2010) 448-458.

[41] K.N. Dahl, P. Scaffidi, M.F. Islam, A.G. Yodh, K.L. Wilson and T. Misteli, Distinct structural and mechanical properties of the nuclear lamina in Hutchinson-Gilford progeria syndrome, Proc Natl Acad Sci U S A 103 (2006) 10271-10276.

[42] A.N. Malhas and D.J. Vaux, Transcription factor sequestration by nuclear envelope components, Cell Cycle 8 (2009) 959-960.

[43] V. Andres and J.M. Gonzalez, Role of A-type lamins in signaling, transcription, and chromatin organization, J Cell Biol 187 (2009) 945-957.

[44] L. Giorgetti, T. Siggers, G. Tiana, G. Caprara, S. Notarbartolo, T. Corona, M. Pasparakis, P. Milani, M.L. Bulyk and G. Natoli, Noncooperative interactions between transcription factors and clustered DNA binding sites enable graded transcriptional responses to environmental inputs, Mol Cell 37 (2010) 418428.

[45] B. Lemon and R. Tjian, Orchestrated response: a symphony of transcription factors for gene control, Genes Dev 14 (2000) 2551-2569.

[46] S.H. Lee and M. Hannink, Molecular mechanisms that regulate transcription factor localization suggest new targets for drug development, Adv Drug Deliv Rev 55 (2003) 717-731.

[47] A.L. Nishimura, V. Zupunski, C. Troakes, C. Kathe, P. Fratta, M. Howell, J.M. Gallo, T. Hortobagyi, C.E. Shaw and B. Rogelj, Nuclear import impairment causes cytoplasmic trans-activation response DNA-binding protein accumulation and is associated with frontotemporal lobar degeneration, Brain 133 (2010) 1763-1771.

[48] C. Wojcik and G.N. DeMartino, Intracellular localization of proteasomes, Int J Biochem Cell Biol 35 (2003) 579-589.

[49] C.H. Eskiw, G. Dellaire and D.P. Bazett-Jones, Chromatin contributes to structural integrity of promyelocytic leukemia bodies through a SUMO-1independent mechanism, J Biol Chem 279 (2004). 
[50] G. Dellaire, R.W. Ching, K. Ahmed, F. Jalali, K.C. Tse, R.G. Bristow and D.P. Bazett-Jones, Promyelocytic leukemia nuclear bodies behave as DNA damage sensors whose response to DNA double-strand breaks is regulated by NBS1 and the kinases ATM, Chk2, and ATR., J Cell Biol 175 (2006) 55.

[51] C.M. de Noronha, M.P. Sherman, H.W. Lin, M.V. Cavrois, R.D. Moir, R.D. Goldman and W.C. Greene, Dynamic disruptions in nuclear envelope architecture and integrity induced by HIV-1 Vpr, Science 294 (2001) 11051108.

[52] H.Y. Kim, C.S. Ki, S.J. Kang, S.K. Khang, S.H. Koh, D.W. Kim, S.H. Kim and I.H. Sung, A novel LMNA gene mutation Leu162Pro and the associated clinical characteristics in a family with autosomal-dominant emery-dreifuss muscular dystrophy, Muscle Nerve 38 (2008) 1336-1339.

[53] J.L. Broers, Y. Raymond, M.K. Rot, H. Kuijpers, S.S. Wagenaar and F.C. Ramaekers, Nuclear A-type lamins are differentially expressed in human lung cancer subtypes, Am J Pathol 143 (1993) 211-220.

[54] C. Stewart and B. Burke, Teratocarcinoma stem cells and early mouse embryos contain only a single major lamin polypeptide closely resembling lamin B, Cell 51 (1987) 383-392.

[55] R.A. Rober, K. Weber and M. Osborn, Differential timing of nuclear lamin $\mathrm{A} / \mathrm{C}$ expression in the various organs of the mouse embryo and the young animal: a developmental study, Development 105 (1989) 365-378.

[56] M.N. Guilly, J.P. Kolb, F. Gosti, F. Godeau and J.C. Courvalin, Lamins A and $\mathrm{C}$ are not expressed at early stages of human lymphocyte differentiation, Exp Cell Res 189 (1990) 145-147.

[57] J.L. Broers, B.M. Machiels, H.J. Kuijpers, F. Smedts, R. van den Kieboom, Y. Raymond and F.C. Ramaekers, A- and B-type lamins are differentially expressed in normal human tissues, Histochem Cell Biol 107 (1997) 505-517.

[58] D. Constantinescu, H.L. Gray, P.J. Sammak, G.P. Schatten and A.B. Csoka, Lamin $\mathrm{A} / \mathrm{C}$ expression is a marker of mouse and human embryonic stem cell differentiation, Stem Cells 24 (2006) 177-185.

[59] Y. Takamori, Y. Tamura, Y. Kataoka, Y. Cui, S. Seo, T. Kanazawa, K. Kurokawa and H. Yamada, Differential expression of nuclear lamin, the major component of nuclear lamina, during neurogenesis in two germinal regions of adult rat brain, Eur J Neurosci 25 (2007) 1653-1662. 


\section{Chapter 5}

\section{Cytoplasmic localization of PML bodies in laminopathies}

F. Houben, W. De Vos, I. Krapels, M. Coorens, G. Kierkels, J. Cox, M. Kamps, V.

Verstraeten, C. Marcelis, A. van den Wijngaard, F.C.S. Ramaekers, J.L.V. Broers

In preparation 


\section{ABSTRACT}

There is growing evidence that laminopathies, diseases associated with mutations in the LMNA gene, are caused by a combination of mechanical and gene regulatory distortions. A striking characteristic of these diseases is the variability in disease symptoms between individual patients carrying an identical LMNA mutation. This is why genetic screening for mutations appears to have little predictive value for disease development. Recently, in laminopathy cells the widespread occurrence of repetitive nuclear ruptures has been described in patient fibroblast cultures. In this study we set out to identify markers, which in a routine setup can identify those cells in which a (recent) nuclear rupture has taken place.

PML nuclear bodies are intranuclear protein structures, which are normally confined to the nuclear interior. Upon nuclear rupture, these structures can leak out of the nucleus and become visible as cytoplasmic PML aggregates. Here we show that a variety of laminopathy cells show the presence of these cytoplasmic PML bodies, and that the amount of these protein aggregates varies with severity of the disease. Also between clinically health individuals, carrying LMNA mutations, significant differences can be found. Therefore we postulate that detection of cytoplasmic PML bodies in patient fibroblasts could become a valuable marker both for screening and prediction of disease development. 


\section{Introduction}

Laminopathies are a group of diseases that result from mutations in the lamin $\mathrm{A} / \mathrm{C}$ gene. The diversity of phenotypes can partly be explained by the different types of mutations affecting LMNA. Laminopathies can be divided into 5 major classes, A) Striated muscle dystrophies, B) Peripheral nerve dystrophies, C) Lipodystrophies, D) Premature ageing syndromes and systemic laminopathies, and E) heterogeneous diseases with overlapping phenotypes [1]. Also, mutations in genes encoding for lamin-associated proteins (e.g. emerin) or enzymes that are responsible for the processing of lamins (e.g. ZMPSTE24) can lead to laminopathies [1]. There are two major hypotheses considered to explain the resulting phenotypes, the 'structural' hypothesis, and the 'gene expression' hypothesis. The first hypothesis states that absence of lamins or incorrect assembly of (mutated) lamins leads to weakening of the nuclear lamina, resulting in both nuclear and cellular weakness. As a result, especially cells, prone to mechanical stress, such as muscle cells, become damaged, resulting in tissue degeneration. The latter hypothesis [2-5] states that the interaction between the nuclear lamina and transcription factors is altered as a result of the mutations in the lamin $\mathrm{A} / \mathrm{C}$ gene. Recent studies indicate that lamin $\mathrm{A} / \mathrm{C}$ mutations or absence of $\mathrm{A}-$ type lamins can provoke a third disease mechanism, i.e. temporary nuclear membrane ruptures, causing inappropriate exchange between cytoplasmic and nuclear components (chapter 4). Upon nuclear membrane rupture (temporary) mobile molecules enter or leave the nucleus, causing the loss of gradient, necessary for maintaining compartment-specific function. Strikingly these rupture are reversible and apparently non-lethal to the cells. Dynamic ruptures of nuclear envelope herniations were previously reported in HIV infected cells, in which the nuclear lamina is compromised [6].

In a previous study we have shown that upon rupture, large protein structures such as PML nuclear bodies (PML NBs) can be lost from the nuclear compartment, and can be detected in the cytoplasm of a considerable percentage of cells (chapter 4).

PML NBs are large intranuclear protein complexes that form functional units containing next to PML, proteins such as Sumo-1, Sp100, p53, pRB, HP1, and Daxx [7]. These bodies are implicated in many cellular functions, including chromatin organization $[8,9]$, viral response (through interferon) [10, 11], DNA replication and repair [12-14], transcriptional regulation $[9,15,16]$, tumor suppression, induction of senescence and apoptosis [7, 17]. 
Sumo-1 modification of the PML protein is necessary for deposition of PML at PML bodies, the recruitment of other associated factors such as Daxx 1 and formation of the PML NBs [18-26]. The purpose of this study was to determine the frequency of cytoplasmic PML NBs in different laminopathy cultures, and to examine the correlation with spontaneous nuclear membrane ruptures. Also, we have investigated whether the relocalization of PML NBs to the cytoplasm is correlated with specific laminopathy subtype and/or progression of the disease. We tested patient cell cultures from the different groups of laminopathies. In one case we were able to test the heterozygous parents of a compound LMNA patient, both of which, are clinically unaffected at this point [27]. We compared the different cell cultures for morphological nuclear abnormalities as an indication for nuclear membrane weakness and compared this with extranuclear PML staining as an indication for efflux of nuclear components. 


\section{Material \& Methods}

\section{Fibroblast cell cultures}

We tested fibroblast cell cultures, derived from patients with laminopathy-related symptoms. Informed consent was obtained from all patients, which were seen for genetic counselling. Fibroblasts were isolated from skin biopsies and subjected to extensive genomic analysis using a specially designed cardio chip [28]. These genomic analyses revealed mutations in LMNA, or ZMPSTE24, in some cases accompanied by mutations in other genes, potentially attributing to the disease phenotype (see below). Cultures, essentially free from epithelial cell contamination were grown in DMEM as described [29] and were passaged at least 3 times before immunocytochemical stainings were performed. Note that for the cell cultures mentioned below, we will refer to them as the abbreviation mentioned (in boldface) for the remaining part of this chapter.

1. As a negative control we used Normal Human Dermal Fibroblasts (NHDF) $\alpha$; Promocell, Heidelberg, Germany).

2. LVNC1 is obtained from a male patient (47 years old) with Left Ventricle Noncompaction with a heterozygous E246R mutation in the lamin A/C gene and an additional A161P mutation in the MYH7 gene.

3. LVNC2 is obtained from a female patient (41 years old) with Left Ventricle Noncompaction with a heterozygous $\mathrm{R} 545 \mathrm{H}$ mutation in the lamin $\mathrm{A} / \mathrm{C}$ gene and an additional L396P mutation in the MYH7 gene.

4. Compound DCM is obtained from a male patient (53 years old) with a compound mutation (R331G and a R627C) in the lamin $\mathrm{A} / \mathrm{C}$ gene.

5. EDMD is obtained from a female patient with Emery-Dreifuss Muscular Dystrophy with a heterozygous I531 deletion in the lamin $\mathrm{A} / \mathrm{C}$ gene.

6. FPLD is obtained from a patient with Familiar Partial Lipodystrophy with a heterozygous $\mathrm{R} 439 \mathrm{C}$ mutation in the lamin $\mathrm{A} / \mathrm{C}$ gene [30].

7. LGMD is obtained from a patient with Limb-Girdle Muscular Dystrophy with a heterozygous $\mathrm{R} 644 \mathrm{C}$ mutation in the lamin $\mathrm{A} / \mathrm{C}$ gene. The patient suffers 
from contractures, sudden loss of strength, and both hypertrophic and dilated cardiomyopathy. The age of onset is about 40 years.

8. HCM is obtained from a male patient (30 years old) with Hypertrophic Cardiomyopathy with ventricular fibrillation, with a heterozygous R644C mutation in the lamin $\mathrm{A} / \mathrm{C}$ gene, a heterozygous $\mathrm{R} 278 \mathrm{C}$ mutation in the TNNT2 gene, a heterozygous Q998R mutation in the MYBPC3 gene and a heterozygous S126G mutation in the GLA gene.

9. RD is obtained from a patient with Restrictive Dermopathy with homozygous c.1085_1086insT truncating mutations in the ZMPSTE24 gene [27]. The patient suffers from a lethal autosomal recessive skin condition characterized by abnormal facies, tight skin, sparse or absent eyelashes, and secondary joint changes.

10. The Compound Progeroid cell culture is obtained from a patient with Compound Progeroid Syndrome with a c.1583C.T (p.T528M) and a c.1619T.C (p.M540T) mutation in the lamin A/C gene [27]. Also, fibroblasts from both the father (M540T) and mother were used. (T528M)

11. HGPS is obtained from a patient with Hutchinson-Gilford Progeria syndrome with a heterozygous $\mathrm{G} 608 \mathrm{G}$ mutation in the lamin $\mathrm{A} / \mathrm{C}$ gene [27].

12. Patient cells with a nonsense homozygous $L M N A$ mutation ((Y259X/Y259X) resulting in absence of lamin $\mathrm{A} / \mathrm{C}$ proteins in these cells. This patient, whose heterozygous family members suffer from limb girdle muscular dystrophy, died at birth [31]. We will refer to theses cells as $\mathbf{H D F}^{\mathbf{m u t} / \mathbf{m u t}}$ cells

\section{Immunocytochemistry}

Human fibroblasts were grown on glass cover slips for at least 48 hours, followed by fixation with $4 \%$ formaldehyde in PBS (15 minutes RT ), followed by permeabilization with Triton X-100 in PBS , (15 min, RT), as described [29]. The following primary antibodies were used:

1. PML (PG-M3) mouse monoclonal antibody Sc-966 (Santa Cruz, Heidelberg, Germany) recognizing different PML isoforms;

2. Rabbit polyclonal antibody recognizing Ki67 (DAKOpatts, Glostrup, DK), diluted 1:100; 
3. Rabbit polyclonal Lamin B specific antibody diluted 1:200, kindly provided by J.C. Courvalin (INSERM. Paris, France)

4. Lamin C specific rabbit polyclonal antibody RalC, (1:20, MUbio Products B.V., Maastricht, The Netherlands);

5. Lamin A/C mouse polyclonal antibody Jol2 (1:50, kindly provided by Prof. C. Hutchison, Durham, UK);

For the detection, the following secundary antibodies were used:

1. FITC conjugated goat anti-rabbit Ig (1:50, SBA/ITK Birmingham, AL, USA);

2. Texas Red conjugated goat anti-rabbit Ig (1:50, SBA/ITK).

3. FITC conjugated rabbit anti mouse Ig antibody (1:100, DAKOpatts, Glostrup, DK)

\section{Confocal microscopy}

Cells were visualized using a Leica TCS SPE confocal laser scanning fluorescence microscope (Leica DMRBE, Mannheim, Germany) as described [32].

\section{PML-distribution}

To test the distribution of PML bodies between the nucleus and the cytoplasm, cells were immunostained (as described above) with PML-antibody. Using fluorescence microscopy, the number of cells with cytoplasmic PML body staining was scored in at least 3 times 100 cells per cell culture.

\section{Transfection and Live Cell Imaging}

Normal as well as laminopathy fibroblasts growing in log phase were transfected with EYFP-NLS [32] (a generous gift from Dr J. Goedhart, University of Amsterdam, the Netherlands), and/or with EYFP-PML [33] using Genejammer (Stratagene, La Jolla, California, USA) [34] according to the manufacturer's instructions. Experiments were performed with cultures ranging from passage 5 to 23 .

No noticeable differences in frequency of nuclear rupture were observed between passages (data not shown). 
Time-lapse recordings were made $24 \mathrm{~h}-48 \mathrm{~h}$ after transfection. Live cell imaging was performed on a Nikon A1R confocal microscope, mounted on a Nikon Ti body, equipped with a Perfect Focus System and a microscope incubator equilibrated at $36.5^{\circ} \mathrm{C}$. Recordings were made using a $60 \mathrm{x} / 1.4$ Plan Apo oil immersion lens. Alternatively, live cell imaging was performed on an inverted fluorescence microscope (Leica DIRBE, Leica Microsystems BV, Rijswijk, The Netherlands), equipped with a black and white CCD camera (CA4742-95, Hamamatsu), a polychrome II polychromator as light source for fluorescence (TILL Photonics, Martinsried, Germany) and a 20x/0.7 Plan Apo lens. 


\section{Results}

\section{PML NBs can be lost from the nucleus upon nuclear rupture}

In the previous chapter, we have discussed the appearance of nuclear ruptures in cells of laminopathy patients. The cells in which the nuclear ruptures were first seen, expressed no lamin A/C (homozygous nonsense mutation). This is an extreme situation (which is not viable, as the patient died shortly after birth), while most other laminopathies contain a mixture of mutated lamin $\mathrm{A} / \mathrm{C}$ and normal lamin $\mathrm{A} / \mathrm{C}$ (with the exception of the compound patients, of which the cells contain only mutated lamin $\mathrm{A} / \mathrm{C}$ protein).

In figure 1, we show a representative cell of the patient with a homozygous lamin $\mathrm{A} / \mathrm{C}$ nonsense mutation cotransfected with EYFP-NLS and EYFP-PML. Twelve minutes after the start of the experiment, the nuclear membrane ruptures, as deduced from the loss of the diffuse intranuclear NLS fluorescence into the cytoplasm, and gradually PML bodies (seen as bright fluorescent spots) flow out of the nucleus into the cytoplasm (arrows). The size of the PML-bodies in the cytoplasm slowly decreases and eventually, after ninety minutes, the PML-bodies have completely disappeared from the cytoplasm.

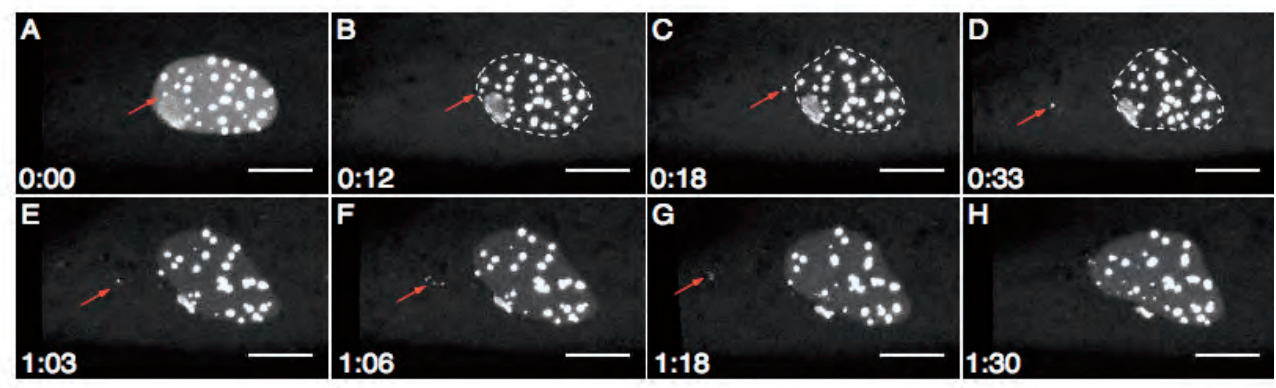

Figure 1: Loss of PML nuclear bodies upon nuclear membrane rupture. Cells, cotransfected with EYFPNLS and EYFP-PML were studied by time-lapse microscopy. Note rupture of the nucleus visible as loss of diffuse intranuclear signal at $\mathrm{t}=0: 12$ followed by escape of PML bodies from the nucleus (arrows), which gradually disappear (1:30). Scale bar $10 \mu \mathrm{m}$.

If this phenomenon could also be seen in other, milder types of laminopathies, it could possibly be used for diagnostic purposes. As vital imaging of NLS-EYFP transfected cell is a time-consuming protocol, and thus not suitable for screening purposes, we 
looked at the presence of PML-bodies in the cytoplasm of cells of different types of laminopathies, and compared these data with other features of these cells.

An overview of all the different cell cultures with their specific mutations, clinical features, percentage of nuclear aberrations, percentage of cytoplasmic PML staining is shown in table 1. Most cell cultures were tested with the EYFP-NLS technique, to visualize the dynamic ruptures, as described in the previous chapter (Chapter 4). In the majority of the tested cell cultures, we were able to demonstrate these reversible ruptures in a live assay (table 1). While nuclear membrane ruptures never occurred in 147 recording of NHDF cells, this event did take place in cell cultures with a LMNA mutation. The number of cells with nuclear ruptures varied considerably between patient cultures, ranging from about 5 to $50 \%$ (table 1 ).

\section{PML localization in laminopathy associated cell cultures}

In order to get insight into the frequency of occurrence of extranuclear PML nuclear bodies, we have performed immunofluorescence on the cell cultures mentioned in table 1. A representative cell of each cell culture, stained for PML-bodies, and the percentage of cells with cytoplasmic PML NBs per cell line is shown in figure 2. In all cell cultures examined a fraction of cells could be detected with cytoplasmic PML bodies next to nucleoplasmic PML NBs. All patient cultures but one (HCM, for a possible explanation see below) show a significantly higher number of cytoplasmic PML NBs than normal cells, ranging from 6 to $60 \%$ in $\mathrm{HDF}^{\mathrm{mut} / \mathrm{mut}}$ cells. In contrast, only about $3 \%$ of normal NHDF cells showed cytoplasmic PML bodies. 


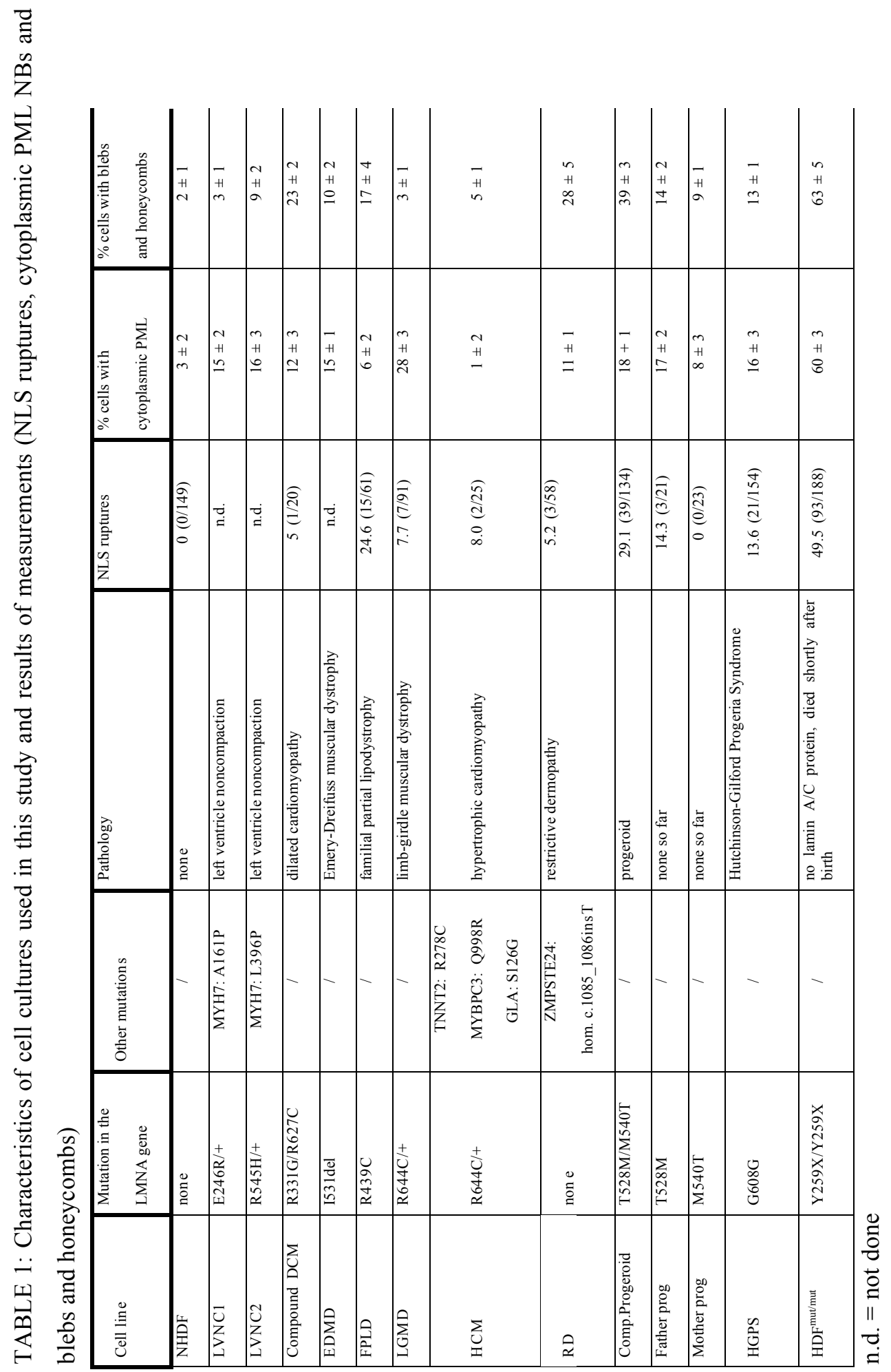




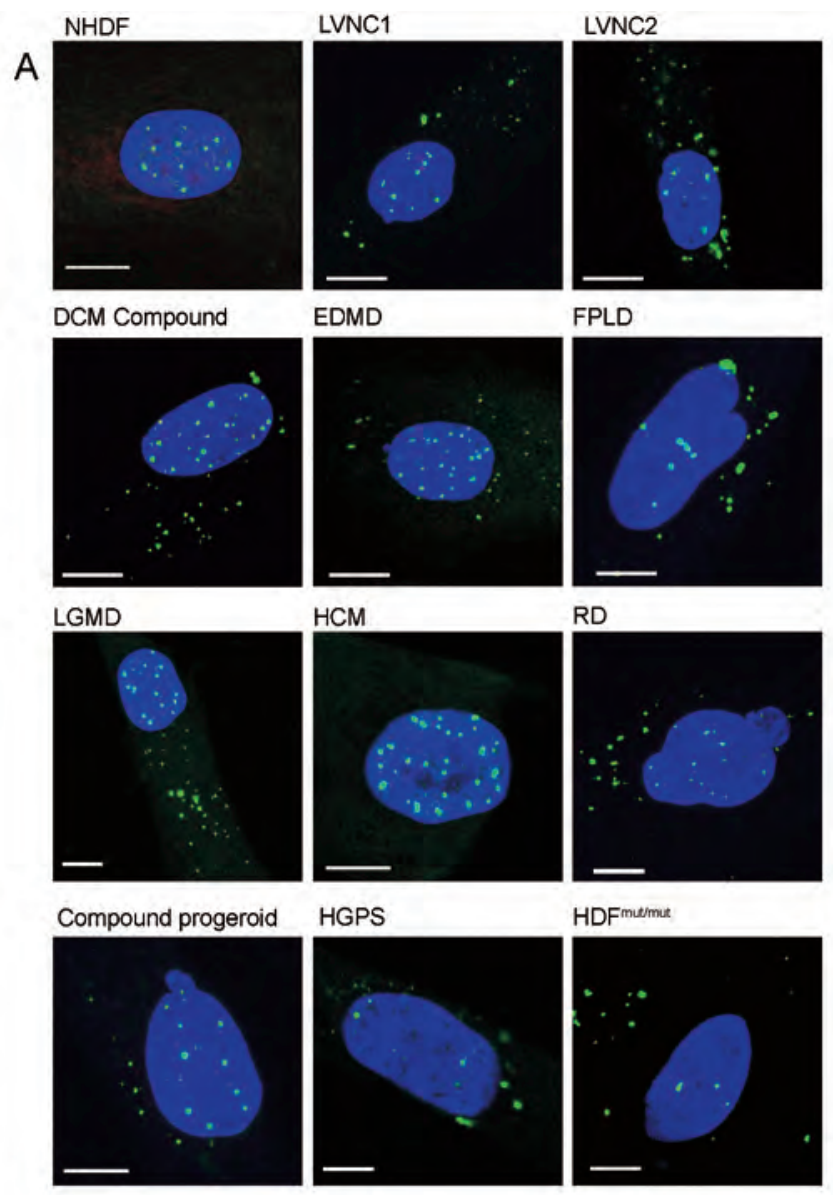

B

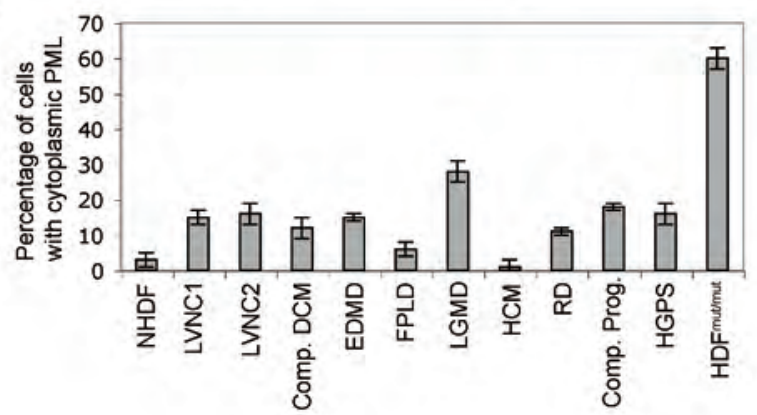

Figure 2: Cytoplasmic localization of PML NBs (panel A) and the percentage of cells with cytoplasmic PML NBs per cell line (panel B). Note that in all fibroblast cultures but NHDF and HDM PML bodies can be found outside of the nucleus. Note also the low amount of intranuclear PML NBs in some of these cells. Scale bar $10 \mu \mathrm{m}$. 


\section{Cytoplasmic localization of PML-NB in laminopathy cells is not caused by mitotic events}

Since it is known that PML NBs relocate tot the cytoplasm during mitosis [35, 36] , and do not re-enter the nucleus immediately after cytokinesis, we have investigated whether the loss of PML NBs in laminopathies is partly due to this mitotic effect. We have performed double immunolabeling with PML and a Ki67 antibody to solve this issue. Ki67 shows a very characteristic immunostaining pattern in early to mid-G1 phase, allowing to identify PML bodies in early G1 cells. Moreover, non-proliferating cells can be distinguished by the absence of the Ki67 protein, allowing discrimination between losses of PML bodies in cycling and non-cycling cells. Figure 3 shows results from these immunolabeling. In NHFD cells cytoplasmic PML bodies could exclusively found in cells in early G-1 phase, No other cycling or noncycling cells showed cytoplasmic PML bodies. In contrast, both the compound progeroid and the HDF ${ }^{\text {mut/mut }}$ cells showed cytoplasmic PML bodies in all interphase stages, including cells that did not express Ki67. While in the compound progeroid cell culture most cells with cytoplasmic PML did express Ki67, especially in the $\mathrm{HDF}^{\mathrm{mut} / \mathrm{mut}}$ cells, cells without Ki67 showed this phenomenon.

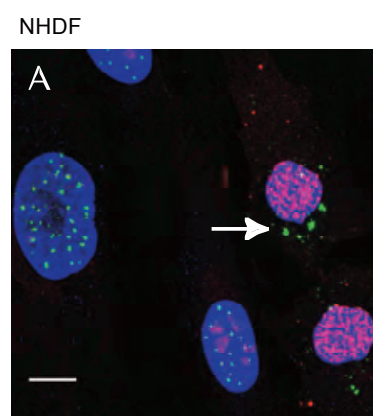

Compund progeroid HDF $^{\text {mut/mut }}$
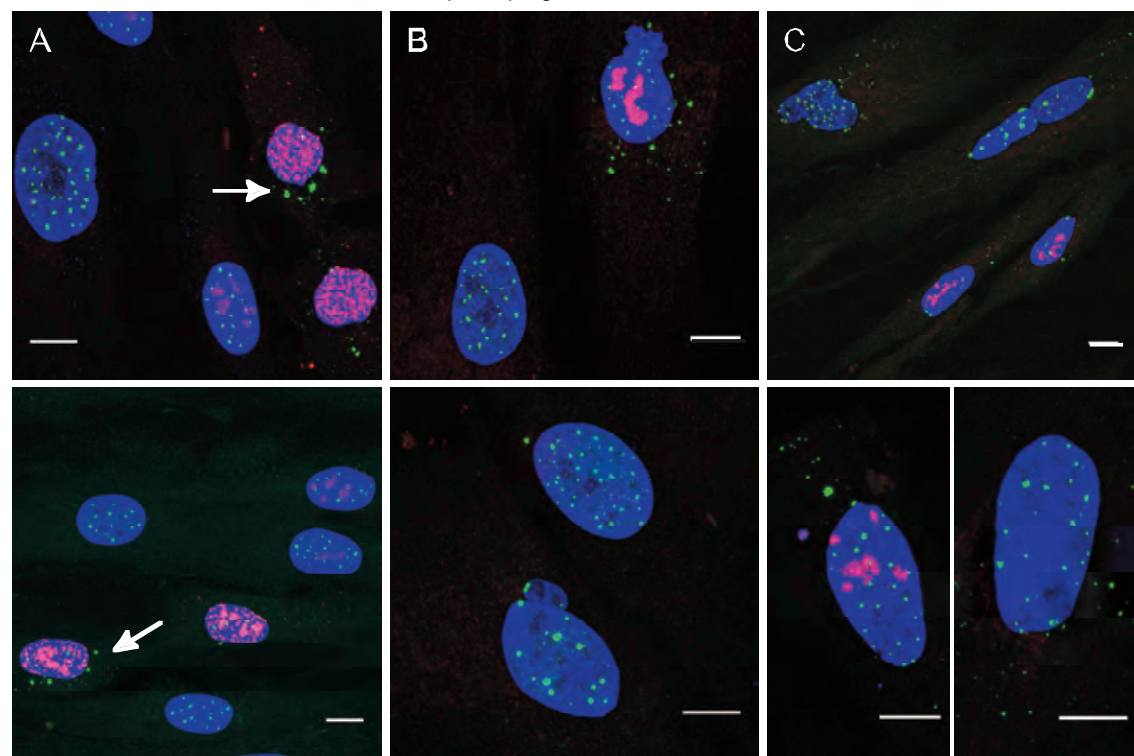

Figure 3: Double immunofluorescence with antibodies against PML (green) and Ki67 (red) of normal NHDF cells (panel A), compound progeroid cells (panel B) and HDF ${ }^{\mathrm{mut} / \mathrm{mut}}$ cells. Note that in the control cell, only cytoplasmic PML staining was found in cells with a characteristic brightly speckled Ki67 labelling (panel A, arrows), while in laminopathy cells cytoplasmic PML bodies were detected in cells with low and even without Ki67 signal (panel B and C). 


\section{Reduced number of PML bodies in part of the laminopathy cells}

From our stainings we got the impression that in cell cultures with high amounts of cytoplasmic PML bodies, a larger variation in nuclear PML NBs could be observed (see also figure 2). Therefore we calculated the amount of PML NBs in the two mostly affected cell cultures and compare these with normal NHDF cells. While the average number of PML NBs did not show large variations between NHDF and compound progeroid cells $(12.3 \pm 7.7$ and $14.0 \pm 9.2$ respectively), the average number for $\mathrm{HDF}^{\mathrm{mut} / \mathrm{mut}}$ cells $(9.6 \pm 9.8)$ was lower. However, due the huge variation in the number of PML bodies per cell, these differences were not significant (p-values (NHDFCompound progeroid $)=0.13$, p-value $\left(\right.$ NHDF- HDF $\left.\left.^{\mathrm{mut} / \mathrm{mut}^{\prime}}\right)=0.08\right)$. However, looking at the frequency distribution of these cultures (figure 4) it becomes clear that while NHDF show a normal distribution, two subpopulations seem to exist in the compound progeroid cell culture, while in the $\mathrm{HDF}^{\mathrm{mut} / \mathrm{mut}}$ cells more cells with very low numbers (1-5) of PML NBs can be found (figure 4).

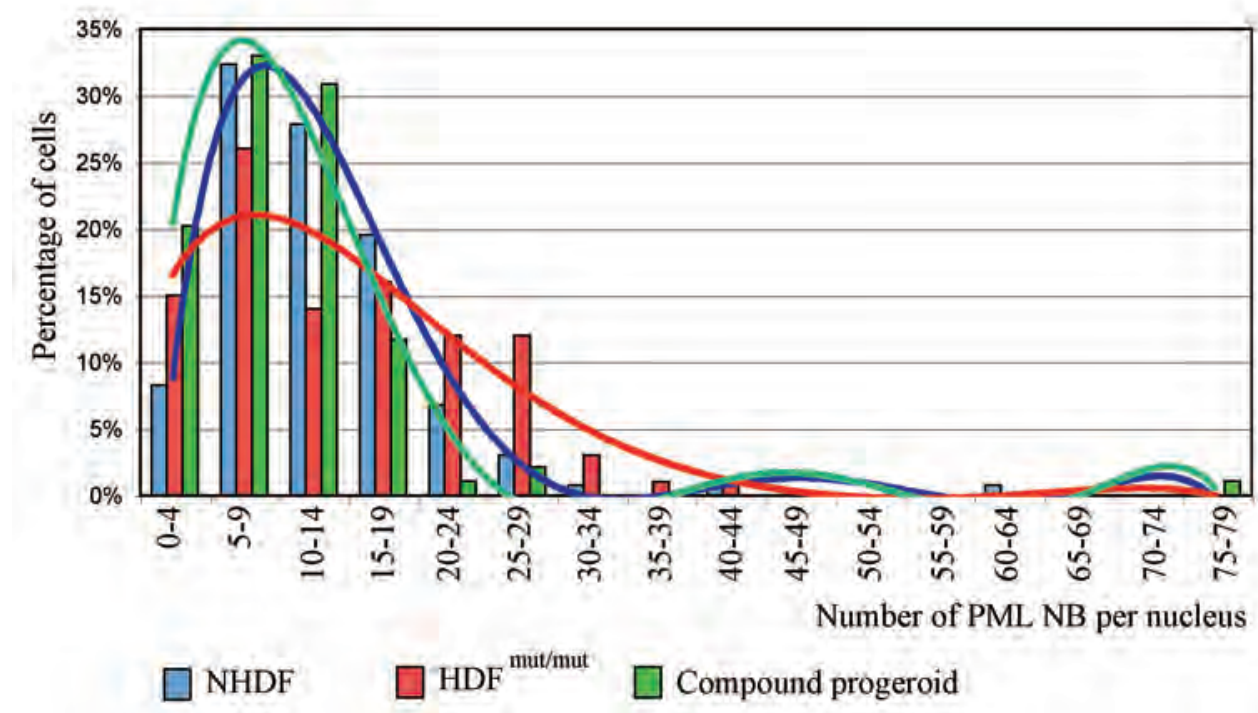

Figure 4: Distribution of the number of nuclear PML NBs per cell in normal (NHDF) and laminopathy cells. 


\section{Not all nuclear ruptures cause visible loss of PML NBs}

The intranuclear organization of PML in nuclear bodies and the association of PML NBS with chromatin made us wonder if a direct correlation between nuclear ruptures and the presence of cytoplasmic PML-NBs could be found.

Normally, most PML NBs are in a higher organized protein complex, tightly bound to the nuclear matrix and/or DNA [7]. In order to detect this correlation, we monitored the accumulative events of nuclear ruptures in cells, by loading dextran in the cytoplasm (see chapter 4). Only a cell, in which a nuclear rupture has occurred in the time period after loading, will show intranuclear dextran fluorescence (Figure 5).

From these experiments it becomes clear that while both dextran containing nuclei and cytoplasmic PLM NBS increase gradually in time (Figure $5 \mathrm{D}$ and 5E), at the cellular level dextran can be present in the nucleus, while PML bodies are not visible in the cytoplasm (Figure 5, panel B). Possibly these PML bodies have been degraded as suggested in the live imaging recordings. Also, the reverse can be occasionally seen, i.e. cytoplasmic PML bodies without intranuclear dextran accumulation (data not shown). These latter data can be explained by the fact that these results were not corrected for the phase of the cell cycle, e.g. in an early G1-phase, not all PML NBS will already be taken up into the nucleus. 


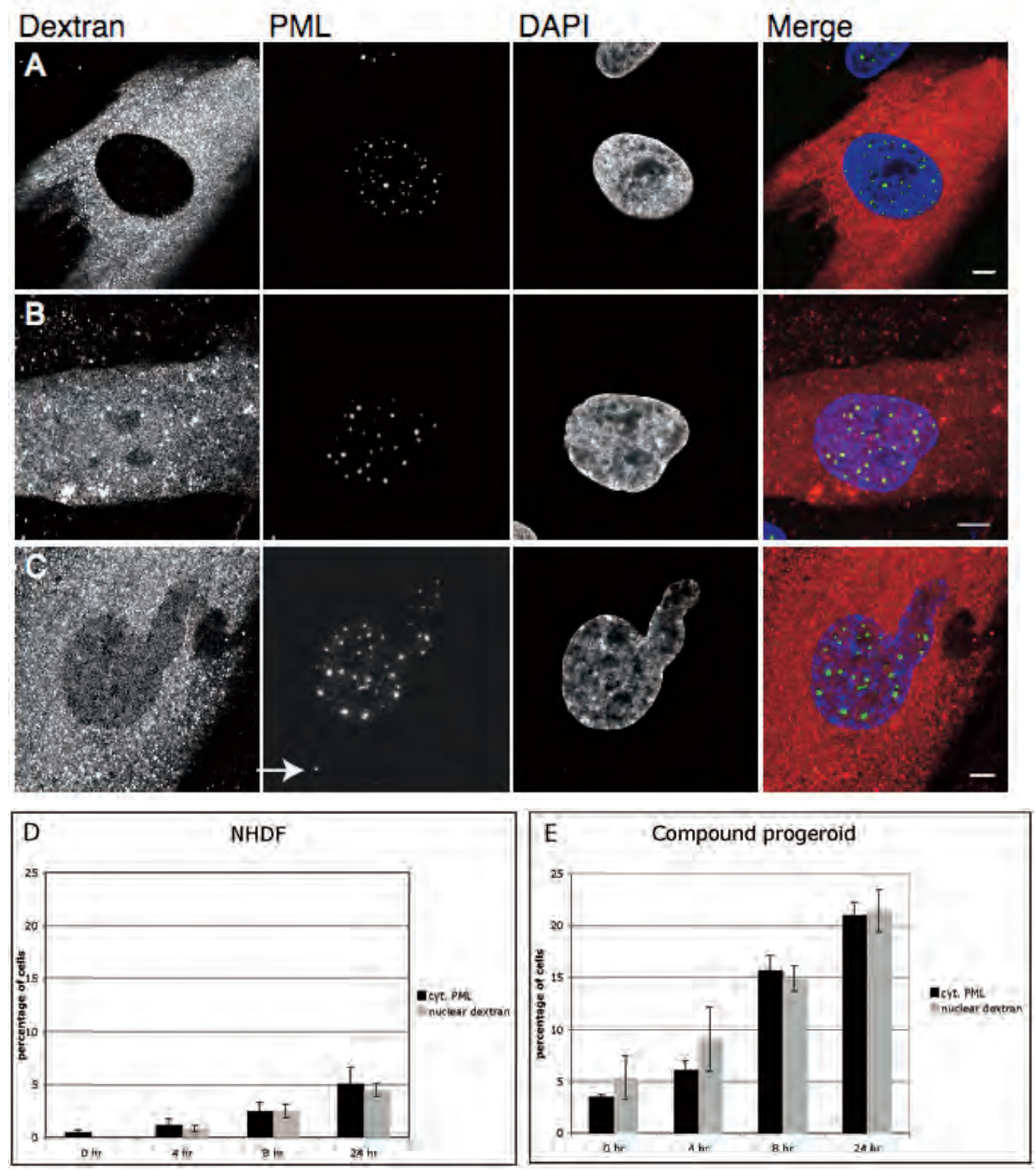

Figure 5: Dextran and PML nuclear body stainings on compound progeroid fibroblasts. Panel A show a cell with an intact nuclear membrane. Sometimes nuclei are filled with dextran without PML nuclear bodies leaving the nucleus (B). Panel C show that PML nuclear bodies can get out of the nucleus (arrow) and dextran into the nucleus at the same time. Next to that, the shape of the nucleus shown in $\mathrm{C}$ is typical for this compound progeroid cell line. D,E: Dextran influx into the nucleus and presence of cytoplasmic PML bodies as detected in wild type fibroblasts (D) and compound progeroid cells (E). Note the large differences in increase of dextran filled nuclei between the two cell cultures for both parameters, while within each cell culture no significant differences between the two parameters are found. 


\section{Correlation between nuclear aberrations, NLS-EYFP ruptures and cytoplasmic PML staining}

The results from the different laminopathy cell lines (nuclear aberrations, the NLSEYFP ruptures and the cytoplasmic PML) were compared to see whether a correlation between the different abnormalities could be seen (figure 6). The cell lines were ordered according the percentage of cells with nuclear aberrations and compared to the results from the control cell line (NHDF). For 4 cell lines, no NLS-GFP experiments could be performed, as indicated in the graph.

While all three types of abnormalities were observed more often than in normal cells, no direct correlation between the three parameters is obvious. Clearly the frequency of cytoplasmic PML bodies does not match with nuclear abnormalities or with nuclear ruptures. As nuclear ruptures occur at spots of the nuclear membrane that are weakened, a correlation between the occurrence of nuclear ruptures, using the EYFPNLS technique and the number of nuclear aberrations (blebs and honeycombs) was to be expected. When we compared the data from the different cell cultures, indeed to some extent a correlation could be seen between the appearance of nuclear ruptures and nuclear aberrations, with the exception of 2 cell cultures (the compound DCM cell line and the RD cell line, of which the latter does not carry a mutation in the LMNA gene, but in the ZMPSTE24 gene.

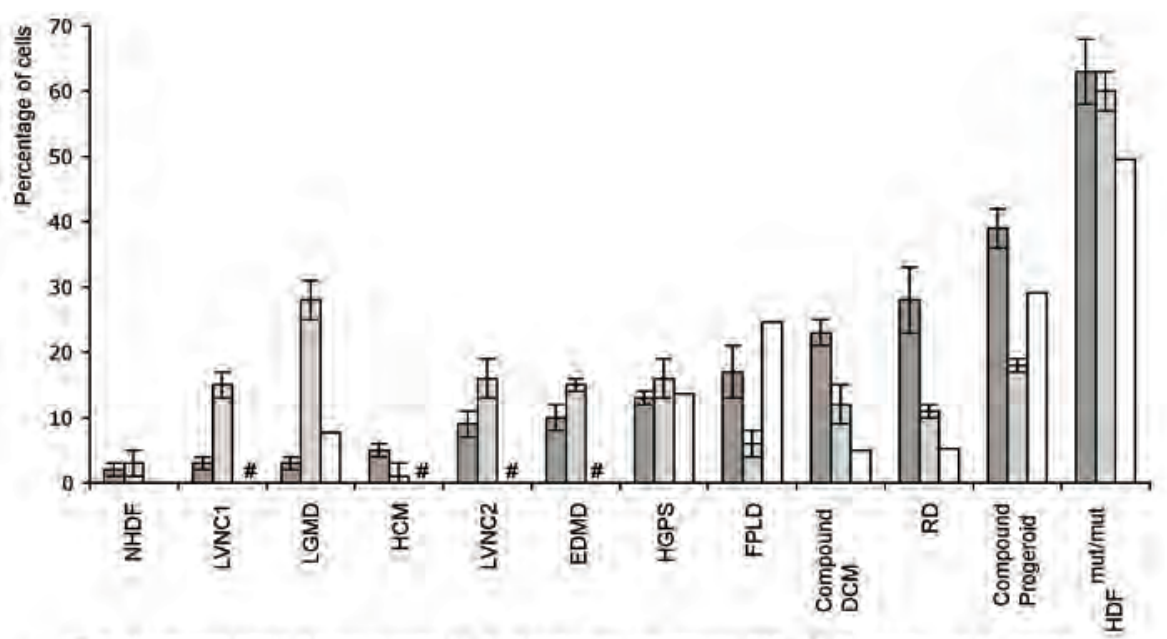

[1 $\%$ of cells with blebs \& honeycombs $\square \%$ of cells with cytoplasmic PML $\square \%$ of cells with NLS-GFP ruptures \# No NLS-GFP results

Figure 6: Comparison between nuclear aberrations, NLS-EYFP ruptures and cytoplasmic PML staining in the same cell cultures 


\section{Cytoplasmic PML bodies can have predictive value for laminopathy development.}

Based on these findings we assume that the presence of PML NBs in the cytoplasm is a potential novel marker for the detection of laminopathies, complementary to nuclear ruptures and nuclear abnormities. In order to evaluate the potential predictive value of this assay we compared fibroblasts of a two-year old male patient with compound heterozygous mutations in the lamin $\mathrm{A} / \mathrm{C}$ gene [27] with his parents. He inherited one mutation from each parent, who is heterozygous for his or her respective mutation. The patient suffers from a progeroid syndrome, resembling symptoms of the classical Hutchinson-Gilford Progeria Syndrome, while both parents are clinically unaffected until now. The father has a c.1583C.T (p.T528M) mutation in exon 9. This mutation is associated in literature with FPLD[35]. The mother carries the c.1619T.C (p.M540T) mutation in exon 10. When we quantified the amount of cells with PML body staining in the nucleus, $17.7 \%$ of the cells of the patient demonstrated cytoplasmic PML bodies. The father had $17.3 \%$ cytoplasmic PML bodies while the mother had only $8.2 \%$ cytoplasmic PML bodies (figure 7). Live cell imaging of transfected fibroblast showed in $14 \%$ of cells from the father $(n=21)$ and $0 \%$ of the cells from the mother $(n=23)$ nuclear ruptures. Also, when comparing the nuclear aberrations, which lead to weak spots in the nuclear lamina, the mother's cells demonstrate fewer aberrations $(8.7 \%)$ compared to the father $(11.3 \%)$ or the patient $(38.8 \%$, figure 7$)$. Both parents have no clinical symptoms at this point, however, the nuclear abnormalities predict development of the disease, especially in the case of the father. Therefore it would be advisable to follow-up the father's clinical condition. 

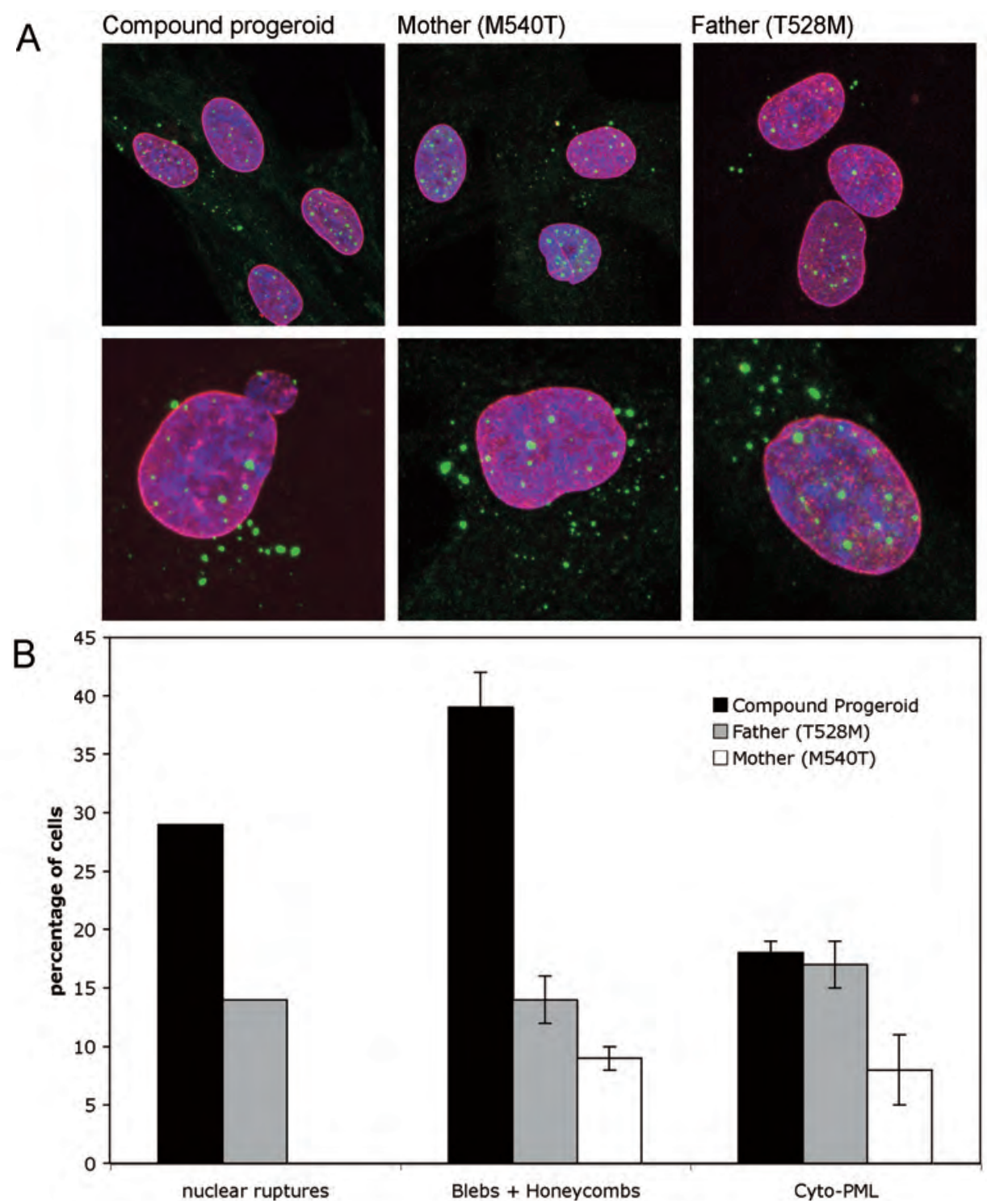

Figure 7: Comparison of cellular abnormalities of the Compound progeroid patient with his parents. A: The distribution of PML bodies in fibroblasts. B: Correlation between nuclear ruptures, the amount of nuclear aberrations, and the presence of cytoplasmic PML bodies in fibroblasts from the patient and both his parents. Scale bar $10 \mu \mathrm{m}$. 


\section{Comparison of cultures with an identical LMNA (R644C)}

mutation

We studied 2 cultures with an identical heterozygous R644C mutation in the LMNA gene. Beside this identical mutation, the cultures did not have a similar genotype or phenotype. The first cell line came from a male patient (about 40 years old) with limbgirdle muscular dystrophy (LGMD) (contractures, sudden loss of strength, and both hypertrophic and dilated cardiomyopathy) but no known mutation in other genes.

The second cell line came from a male patient (30 years old) with hypertrophic cardiomyopathy (HCM) with ventricular fibrillation and with additional mutations in the TNNT2, the MYBPC3 and the GLA gene.

When we compared the percentage of cells with cytoplasmic PML bodies, the difference between the two cell cultures was dramatic (LGMD: $28 \pm 3 \%$; HCM $1 \pm 2$ $\%$ ), although the number of cells with nuclear aberrations in both cultures was comparable (LGMD: $3 \pm 1 \%$; HCM $5 \pm 1 \%$ ). Based on the absence of cytoplasmic PM-NBs as well as the absence of lamin or nuclear weak spots, we now consider this HCM patient having no laminopathy, but having clinical symptoms due to the mutations in one or some of the other genes. 


\section{DISCUSSION}

Following the description of the process of the dynamic ruptures in lamin-deficient cells in the previous chapter, which lead to the translocation of macromolecular complexes such as PML-bodies, we wanted to test whether this translocation of PML bodies to the cytoplasm was commonly seen in different types of laminopathies. While live-imaging of NLS-EYFP transfected cells is the most direct manner to determine whether dynamic ruptures of the nuclear membrane in living cells occur (chapter 4), it is, however, too time-consuming and difficult to perform in a routine screening setup. Furthermore, a serious drawback of this method is the limited number of cells within a cell population that can be screened. Since the duration of rupture visibility can be very short (a few minutes), it is not possible to monitor large numbers of cells simultaneously. Therefore a reliable statistical analysis of nuclear rupture events is not possible sing out imaging setup. In our attempts to search for markers which would allow alternative quantification methods for registration of nuclear ruptures we came across PML nuclear bodies, which, under normal growth conditions are almost completely confined to the nucleus (for a recent review see [36]). While PML proteins can be present in the cytoplasm, only in case of mutations in the PML gene larger protein complexes called cytoplasmic PML bodies can form [37]. Since hitherto nuclear ruptures have only been described in laminopathies and HIV- infected cells (due to lamina breakdown [6]), registration of the translocation of PML bodies due to nuclear ruptures may be used as a diagnostic marker for laminopathies.

Cytoplasmic PML staining in the normal NHDF cells was not zero. Some cells (about $3 \%$ in unsynchronised cultures) did show cytoplasmic PML aggregates. This could be explained by the cell-cycle-dependent presence of cytoplasmic PML structures [38]. Indeed, we were able to show that all of the cells with cytoplasmic PML bodies found in NHDF showed a typical staining pattern for Ki67, indicating early G1-phase of these cells. The number of cytoplasmic PML bodies could be reduced to zero after serum starvation and increased to more than $20 \%$ after cycle synchronisation (data not shown). These findings match very well with previous studies in other normal cell cultures $[36,38]$. The data from the current PML-study indicate that not only in lamindeficient cells (which are extremely rare, so clinically less relevant), but also in cultures from laminopathy patients, cytoplasmic PML bodies can be seen. In the lamin $\mathrm{A} / \mathrm{C}$-deficient cells ( $\mathrm{HDF}^{\mathrm{mut} / \mathrm{mut}}$ ), up to $60 \%$ of the cells have cytoplasmic PML 
staining. Also in the other laminopathies cytoplasmic PML bodies could be detected (up to in $28 \%$ of all cells),

While the percentage of nuclear ruptures cannot be correlated to the amount of cells with cytoplasmic PLM bodies, possibly due to the low number cells in which nuclear ruptures were registered, it became evident that in general in cells with at least one nuclear rupture, significantly higher than normal numbers of cytoplasmic PML bodies could be found. Since we did evaluate a relative large number of normal NHDF $(n=149)$ and never found a nuclear rupture in these cells, we can state that indeed such a rupture is extremely rare, or even absent. The only exception is a diseased cell culture showing 2/25 nuclear ruptures, but no cytoplasmic PLM bodies, and no nuclear or lamin abnormalities, indicating that this patient probably does not suffer from a laminopathy (see below).

It is conceivable, that nuclear ruptures are more common in cells with visible nuclear aberrations, which by immunofluorescence can be seen as irregularities in nuclear shape, or variations in intensity and pattern of lamin immunolabeling. In order to investigate such a correlation, we compared the number of cells containing cytoplasmic PML with the number of cells having nuclear aberrations (blebs, honeycombs, and other visible weak spots of the nuclear lamina). However, no overall correlation could be seen. Also at the individual cell level, no such a correlation could be established: cells with apparently large nuclear deformations showed no cytoplasmic PML bodies, while cells with apparently normal looking nuclei showed a massive loss of PML NBs into the cytoplasm. While several explanations for this discrepancy are possible, this does indicate that the presence of cytoplasmic PML bodies could be an independent parameter for the detection of laminopathies. In this respect it is promising to notice that the most severe cases of laminopathies (compound progeroid, HGPS, $\mathrm{HDF}^{\mathrm{mut} / \mathrm{mut}}$ appear to show a relatively high number of cytoplasmic PML bodies.

Additional indications that indeed cytoplasmic PML bodies result from laminopathies and, reversely, can have a predictive value for the disease development come from our studies on the compound progeroid patient and his parents. The diseased child has a high number of nuclear ruptures and cytoplasmic PML bodies, significantly higher than his healthy mother, Strikingly, his symptom - free father has also relatively high numbers of ruptures and cytoplasmic PML bodies, predicting development of laminopathy-related disease symptoms in time. This age of this father (now 32 years old) is relatively young for laminopathy development in patients with this 
heterozygous mutation. Thus, continued clinical follow-up of the father's health status is highly recommended.

Another interesting case is the difference in clinical outcome in the 2 patients with the same R644C mutation. Previous studies show that the R644C mutation can lead to extreme phenotypic diversity and non-penetrance. The difference in clinical outcome can be explained by a second not yet identified mutation in a functionally linked proteins (e.g. emerin, desmin) [39-41]. In these cell cultures examined, the differences in the markers for the loss of nuclear integrity may point to an negative effect of the LMNA mutation in the LGMD patient, while the negative effects on the phenotype is the HCM patient are more likely to be caused by the mutations in 3 other genes in this patient. This indicates that testing for loss of nuclear integrity in patients and family members with this mutation could be a possible predictor for the rate of penetrance of the mutation.

The exact origin of the cytoplasmic PML bodies is currently under investigation. While vital imaging clearly showed that ruptures of the nuclear membrane (as shown in chapter 4) could lead to the efflux of individual PML-bodies, it is premature to state that all cytoplasmic PML bodies arise from these ruptures. Dextran labelled cells occasionally showed cytoplasmic PML bodies without signs of dextran influx, indicating nuclear rupture, while also the reverse could be observed. Several factors could have a negative effect on this correlation. First, the nuclear rupture could have a short duration or could only cause a minor opening in the nuclear membrane allowing small molecules (NLS) to escape, but not the larger PML NBs. Secondly, most PML NBs are normally tightly bound to the nuclear matrix, including DNA, possibly preventing the escape of PML NBs from the nucleus. Absence of PML bodies from the cytoplasm in apparently ruptured nuclei can be explained by our observation in live imaging experiments that PML bodies seen to be degraded after relocalization to the cytoplasm (Figure 1). However, it is also possible that other cellular processes can lead to cytoplasmic PML-staining. When we immunocytochemically stained the cytoplasmic PML bodies, we observed that these aggregates contain no SUMO-1, Daxx or SP-100 (as shown in chapter 4). Research from Ishov and Butler describe cytoplasmic PML aggregates as immature PML bodies, with lack SUMO-1, Daxx and Sp200 [22, 42]. As the sumoylation of PML is essential for the correct formation of PML bodies (with all the associated proteins), a mutation in lamin $\mathrm{A} / \mathrm{C}$ can influence the formation and localization of PML bodies and can lead to immature PML bodies in the cytoplasm of cells. 
Lamins influence the correct localization and functioning of SUMO-1 [43]. Certain mutations in the lamin $\mathrm{A} / \mathrm{C}$ gene lead to an abnormal distribution of SUMO-1 and a reduction in sumoylation of proteins. Vice versa, the lamin protein is also sumoylated, rather by SUMO-2 than by SUMO-1[44]. A defect in lamin A/C sumoylation could play an important role in the underlying molecular mechanism of the familial cardiomyopathies associated laminopathies. This disturbance of sumoylation of PMLbodies, together with the phenomenon of the nuclear ruptures could thus also explain the cytoplasmic PML-bodies seen in cells from laminopathy patients. Currently we can only speculate on the effect of loss of (functional) PML NBS in laminopathies. The diversity of functions, assigned to these structures allows speculating into several directions. The interaction of both lamins and PML nuclear bodies with transcription factors has long been recognized. Loss of functionality of both complexes could have a complementary effect, causing a variety of diseases. Also, the partial loss of DNA repair capacity in laminopathies could be explained by non-functioning PML NBs, known to be important sensors for DNA repair [14].

The results from the different cultures, and especially the progeroid patient and both his parents clearly show that the phenomenon of cytoplasmic PML-staining is a potential method for screening not only possible patients, but also family members of laminopathy patients which show no clinical phenotype at first sight. This screening could be used to test whether the parents and siblings of the patient are at risk for developing clinical symptoms, or are merely subclinical and thus likely tot develop symptoms over the next couple of years. In the latter case, further follow-up and preventive measures can then be provided.

Taken together, these results indicate that the loss of nuclear integrity by measuring the percentage of cell with cytoplasmic PML is a potential novel diagnostic tool in detecting laminopathies. Such markers are needed in view of the poor predictive value of both genetic screening and of detection of nuclear aberrations [45], Clearly many more cases have to be evaluated in order to asses its value both as a prognostic and a potential prognostic marker. 


\section{REFERENCES}

[1] J.L. Broers, F.C. Ramaekers, G. Bonne, R.B. Yaou and C.J. Hutchison, Nuclear lamins: laminopathies and their role in premature ageing, Physiol Rev 86 (2006) 967-1008.

[2] M. Cohen, K.K. Lee, K.L. Wilson and Y. Gruenbaum, Transcriptional repression, apoptosis, human disease and the functional evolution of the nuclear lamina, Trends Biochem Sci 26 (2001) 41-47.

[3] K.L. Wilson, M.S. Zastrow and K.K. Lee, Lamins and disease: insights into nuclear infrastructure, Cell 104 (2001) 647-650.

[4] S. Vlcek and R. Foisner, A-type lamin networks in light of laminopathic diseases, Biochim Biophys Acta 1773 (2007) 661-674.

[5] E.C. Schirmer and R. Foisner, Proteins that associate with lamins: many faces, many functions, Exp Cell Res 313 (2007) 2167-2179.

[6] C.M. de Noronha, M.P. Sherman, H.W. Lin, M.V. Cavrois, R.D. Moir, R.D. Goldman and W.C. Greene, Dynamic disruptions in nuclear envelope architecture and integrity induced by HIV-1 Vpr, Science 294 (2001) 11051108 .

[7] R. Bernardi and P.P. Pandolfi, Structure, dynamics and functions of promyelocytic leukaemia nuclear bodies, Nat Rev Mol Cell Biol 8 (2007) 1006-1016.

[8] J.S. Seeler and A. Dejean, The PML nuclear bodies: Actors or extras?, Curr Opin Genet Dev 9 (1999) 362-367.

[9] F.M. Boisvert, K. M.J., A.K. Box, M.J. Hendzel and D.P. Bazett-Jones, The transcription coactivator CBP is a dynamic component of the promyelocytic leukemia nuclear body., J Cell Biol 152 (2001) 1099-1106.

[10] A.M. Ishov and G.G. Maul, The periphery of nuclear domain 10 (ND10) as site of DNA virus deposition, J Cell Biol 134 (1996) 815-826.

[11] T. Regad and M.K. Chelbi-Alix, Role and fate of PML nuclear bodies in response to interferon and viral infections, Oncogene 20 (2001) 7274-7286.

[12] S. Zhong, P. Hu, T.Z. Ye, R. Stan, N.A. Ellis and P.P. Pandolfi, A role for PML and the nuclear body in genomic stability, Oncogene 18 (1999) 79417947.

[13] C.H. Eskiw, G. Dellaire and D.P. Bazett-Jones, Chromatin contributes to structural integrity of promyelocytic leukemia bodies through a SUMO-1independent mechanism, J Biol Chem 279 (2004).

[14] G. Dellaire, R.W. Ching, K. Ahmed, F. Jalali, K.C. Tse, R.G. Bristow and D.P. Bazett-Jones, Promyelocytic leukemia nuclear bodies behave as DNA damage sensors whose response to DNA double-strand breaks is regulated by NBS1 and the kinases ATM, Chk2, and ATR., J Cell Biol 175 (2006) 55.

[15] W.S. Wu, S. Vallian, E. Seto, W.M. Yang, D. Edmondson, S. Roth and K.S. Chang, The growth suppressor PML represses transcription by functionally 
and physically interacting with histone deacetylases, Mol Cell Biol 21 (2001) 2259-2268.

[16] J. Wang, C. Shiels, P. Sasieni, P.J. Wu, S.A. Islam, P.S. Freemont and D. Sheer, Promyelocytic leukemia nuclear bodies associate with transcriptionally active genomic regions, J Cell Biol 164 (2004) 515-526.

[17] O. Bischof, O. Kirsh, M. Pearson, K. Itahana, P.G. Pelicci and A. Dejean, Deconstructing PML-induced premature senescence, EMBO 21 (2002).

[18] T. Sternsdorf, K. Jensen and H. Will, Evidence for covalent modification of the nuclear dot-associated proteins PML and Sp100 by PIC1/SUMO-1, J Cell Biol 139 (1997) 1621-1634.

[19] V.J. LaMorte, J.A. Dyck, R.L. Ochs and R.M. Evans, Localization of nascent RNA and CREB binding protein with the PMLcontaining nuclear body, Proc Natl Acad Sci U S A 95 (1998) 4991-4996.

[20] T. Kamitani, K. Kito, H.P. Nguyen, H. Wada, T. Fukada-Kamitami and E.T. Yeh, Identification of three major sentrinization sites in PML, J Biol Chem 273 (1998) 26675-26682.

[21] S. Muller, M.J. Matunis and A. Dejean, Conjugation with the ubiquitinrelated modifier SUMO-1 regulates the partitioning of PML within the nucleus, EMBO 17 (1998) 61-70.

[22] A.M. Ishov, A.G. Sotnikov, D. Negorev, O.V. Vladimirova, N. Neff, T. Kamitani, E.T. Yeh, J.F. Strauss III and G.G. Maul, PML Is Critical for ND10 Formation and Recruits the PML-interacting Protein Daxx to this Nuclear Structure When Modified by SUMO-1, J Cell Biol 147 (1999) 221-233.

[23] S. Zhong, P. Salomoni and P.P. Pandolfi, The transcriptional role of PML and the nuclear body., Nat Cell Biol 2 (2000) E85-E90.

[24] V. Lallemand-Breitenbach, J. Zhu and F. Puvion, Role of promyelocytic leukemia (PML) sumolation in nuclear body formation, 11S proteasome recruitment, and As2O3-induced PML or PML/retinoic acid receptor alpha degradation, J Exp Med 193 (2001) 1361-1371.

[25] T.H. Shen, H.K. Lin, P.P. Scaglioni, T.M. Yung and P.P. Pandolfi, The mechanisms of PML-nuclear body formation, Mol Cell 24 (2006) 331-339.

[26] S. Nagai, N. Davoodi and S.M. Gasser, Nuclear organization in genome stability: SUMO connections, Cell Res (2011) 1-12.

[27] V.L. Verstraeten, J.L. Broers, M.A. van Steensel, S. Zinn-Justin, F.C. Ramaekers, P.M. Steijlen, M. Kamps, H.J. Kuijpers, D. Merckx, H.J. Smeets, R.C. Hennekam, C.L. Marcelis and A. van den Wijngaard, Compound heterozygosity for mutations in LMNA causes a progeria syndrome without prelamin A accumulation, Hum Mol Genet 15 (2006) 2509-2522.

[28] A. Van den Wijngaard, W. Van Dijk, A.P. Stassen, P.J. Lindsey, Y.H. Arens, P. Helderman, C.L. Marcelis, J.J. Van der Smagt, S. Heymans, P.G. Volders, R.J. Jongbloed and H.J. Smeets, CHIP-based Sequence Analysis of 34 Cardiomyopathy Genes Reveals New Genes Involved in HCM and DCM and 
Multiple Pathogenic Mutations in Single Patients Circulation 120 (2009) S565.

[29] F. Houben, C.H. Willems, I.L. Declercq, K. Hochstenbach, M.A. Kamps, L.H. Snoeckx, F.C. Ramaekers and J.L. Broers, Disturbed nuclear orientation and cellular migration in A-type lamin deficient cells, Biochim Biophys Acta 1793 (2009) 312-324.

[30] V.L. Verstraeten, S. Caputo, M.A. van Steensel, I. Duband-Goulet, S. ZinnJustin, M. Kamps, H.J. Kuijpers, C. Ostlund, H.J. Worman, J.J. Briede, C. Le Dour, C.L. Marcelis, M. van Geel, P.M. Steijlen, A. van den Wijngaard, F.C. Ramaekers and J.L. Broers, The R439C mutation in LMNA causes lamin oligomerization and susceptibility to oxidative stress, J Cell Mol Med 13 (2009) 959-971.

[31] A. Muchir, B.G. van Engelen, M. Lammens, J.M. Mislow, E. McNally, K. Schwartz and G. Bonne, Nuclear envelope alterations in fibroblasts from LGMD1B patients carrying nonsense Y259X heterozygous or homozygous mutation in lamin A/C gene, Exp Cell Res 291 (2003) 352-362.

[32] G.J. Kremers, J. Goedhart, E.B. van Munster and T.W. Gadella, Jr., Cyan and yellow super fluorescent proteins with improved brightness, protein folding, and FRET Forster radius, Biochemistry 45 (2006) 6570-6580.

[33] K. Wiesmeijer, C. Molenaar, I.M. Bekeer, H.J. Tanke and R.W. Dirks, Mobile foci of Sp100 do not contain PML: PML bodies are immobile but PML and Sp100 proteins are not, J Struct Biol 140 (2002) 180-188.

[34] W.H. De Vos, F. Houben, M. Kamps, A. Malhas, F. Verheyen, J. Cox, E.M. Manders, V.L. Verstraeten, M.A. van Steensel, C.L. Marcelis, A. van den Wijngaard, D.J. Vaux, F.C. Ramaekers and J.L. Broers, Repetitive disruptions of the nuclear envelope invoke temporary loss of cellular compartmentalization in laminopathies, Hum Mol Genet 20 (2011) 41754186.

[35] D.B. Savage, M.A. Soos, A. Powlson, S. O'Rahilly, I. McFarlane, D.J. Halsall, I. Barroso, E.L. Thomas, J.D. Bell, I. Scobie, P.E. Belchetz, W.F. Kelly and A.J. Schafer, Familial partial lipodystrophy associated with compound heterozygosity for novel mutations in the LMNA gene, Diabetologia 47 (2004) 753-756.

[36] A. Carracedo, K. Ito and P.P. Pandolfi, The nuclear bodies inside out: PML conquers the cytoplasm, Curr Opin Cell Biol 23 (2011) 360-366.

[37] C. Bellodi, K. Kindle, F. Bernassola, D. Dinsdale, A. Cossarizza, G. Melino, D. Heery and P. Salomoni, Cytoplasmic function of mutant promyelocytic leukemia (PML) and PML-retinoic acid receptor-alpha, J Biol Chem 281 (2006) 14465-14473.

[38] A. Jul-Larsen, A. Grudic, R. Bjerkvig and S.O. Boe, Cell-cycle regulation and dynamics of cytoplasmic compartments containing the promyelocytic leukemia protein and nucleoporins, J Cell Sci 122 (2009) 1201-1210. 
[39] E. Mercuri, S.C. Brown, P. Nihoyannopoulos, J. Poulton, M. Kinali, P. Richard, R.J. Piercy, S. Messina, C. Sewry, M.M. Burke, W. McKenna, G. Bonne and F. Muntoni, Extreme variability of skeletal and cardiac muscle involvement in patients with mutations in exon 11 of the lamin $\mathrm{A} / \mathrm{C}$ gene, Muscle Nerve 31 (2005) 602-609.

[40] F. Muntoni, G. Bonne, L.G. Goldfarb, E. Mercuri, R.J. Piercy, M. Burke, R.B. Yaou, P. Richard, D. Recan, A. Shatunov, C.A. Sewry and S.C. Brown, Disease severity in dominant Emery Dreifuss is increased by mutations in both emerin and desmin proteins, Brain 129 (2006) 1260-1268.

[41] J. Rankin, M. Auer-Grumbach, W. Bagg, K. Colclough, T.D. Nguyen, J. Fenton-May, A. Hattersley, J. Hudson, P. Jardine, D. Josifova, C. Longman, R. McWilliam, K. Owen, M. Walker, M. Wehnert and S. Ellard, Extreme phenotypic diversity and nonpenetrance in families with the LMNA gene mutation R644C, Am J Med Genet A 146A (2008) 1530-1542.

[42] J.T. Butler, L.L. Hall, K.P. Smith and J.B. Lawrence, Changing Nuclear Landscape and Unique PML Structures During Early Epigenetic Transitions of Human Embryonic Stem Cells, J Cell Biochem 107 (2009) 609-621.

[43] N. Sylvius, Z.T. Bilinska, J.P. Veinot, A. Fidzianska, P.M. Bolongo, S. Poon, P. McKeown, R.A. Davies, K.-L. Chan, A.S.L. Tang, S. Dyack, J. Grzybowski, W. Ruzyllo, H. McBride and F. Tesson, In vivo and in vitro examination of the functional significances of novel lamin gene mutations in heart failure patients, J Med Genet 42 (2005) 639-647.

[44] Y.Q. Zhang and K.D. Sarge, Sumoylation regulates lamin A function and is lost in lamin A mutants associated with familial cardiomyopathies, J Cell Biol 182 (2008) 35-39.

[45] A. Muchir, J. Medioni, M. Laluc, C. Massart, T. Arimura, A.J. van der Kooi, I. Desguerre, M. Mayer, X. Ferrer, S. Briault, M. Hirano, H.J. Worman, A. Mallet, M. Wehnert, K. Schwartz and G. Bonne, Nuclear envelope alterations in fibroblasts from patients with muscular dystrophy, cardiomyopathy, and partial lipodystrophy carrying lamin A/C gene mutations, Muscle Nerve 30 (2004) 444-450. 


\section{Summary and General Discussion}


Not long after the first discoveries of LMNA mutations causing two different diseases [1], it was recognized that mutations in the LMNA gene cause a variety of diseases, collectively called laminopathies. While the number of described pathologies has risen exponentially, the clinical diversity of the diseases associated with LMNA mutations has raised numerous questions about the underlying disease development mechanisms at the (sub)cellular level. The first obvious question is how different mutations in a single gene can cause this variety of diseases. Extensive protein structural analyses did not solve this question yet, especially since the exact function of affected protein domains is not well known. Lamin A/C proteins form homo-and heterodimers, forming the nuclear lamina, which suggested functions are: 1) formation of a barrier between the nucleus and cytoplasm; 2) providing structural support to the nucleus; 3 ) aiding in nucleus reformation after mitosis; 4) interaction with several intranuclear and transmembrane proteins, forming both a mechanical scaffold, as well as a docking site for signaling proteins; 5) interaction with chromatin, suggesting several (sub-) functions: $i$ - chromatin organisation, formation of heterochromatin; ii - chromatin replication; iii - gene regulation .

A second important question is why identical mutations lead to different disease symptoms between patients: Identical mutations can lead to FPLD or to EDMD or other clinically distinct phenotypes such as DCM. Moreover the disease penetrance can be extremely variable, even between family members. Siblings with identical mutations can either develop a severe form of laminopathy or remain without disease symptoms throughout their lifespan. This makes it virtually impossible to predict clinical outcome even if the genetic diagnosis confirms the presence of a LMNA mutation.

While several studies have focused on gene regulatory effects of LMNA mutations, this thesis describes the structural defects in lamina organisation and the resulting deficiencies in functioning, due to either absence of lamins or LMNA mutations.

In chapter 1, the impact of the absence of A-type lamins on the integrity of the cellular structural scaffold was described. This network consists of the nuclear lamina (lamins and associated proteins), the cytoskeletal proteins and cell adhesion molecules. All these proteins are interconnected through linker proteins. At the nuclear envelope, SUN-proteins and nesprins link the nuclear lamina to the different cytoskeletal elements, which in turn are connected to the cellular membrane via actinin, catenin, vinculin, plectin and other membrane linker proteins. The network as a whole forms a scaffold that acts as a tensegrity structure. This structure combines internal compression (microtubules) and tension (actin filaments) forces and protects the 
cellular components (like the nucleus) during mechanical compression [2-5]. All the elements of this mechanical network are essential as they form one interacting mechanical entity, which cannot function if one of the elements fails. When the lamin structure is disturbed (as a result of a mutation or knock-out), this has a dramatic effect on the other elements of the network. Lamin-associated proteins (e.g. emerin), or linker-proteins (e.g. nesprins) are mislocalized when lamin A/C proteins are affected. Previous related studies showed that upon nuclear compression, cells without A-type lamins show nuclear rupture while these cells also showed a reduced resistance to force generated by compression [6]. Extension of these studies on isolated nuclei (with or without A-type lamins) showed that indeed the intact cellular scaffold is needed to generate cellular strength, since all types of isolated nuclei showed similar deformation upon compression.

These findings suggest that other defects in elements of the cytoskeleton and linker proteins can lead to a dysfunctional mechanical network at the cellular level, leading to comparable clinical features (see below).

In chapter 2, we were able to show that loss of the integrity of the mechanical scaffold leads to a loss of functional mechanical properties of the cell. The correct positioning of the nucleus and the MTOC is essential for the cell migration process [7, 8]. This repositioning depends on the mechanical scaffold network. As this network fails, as a result of lamin deficiency, the nucleus and the MTOC cannot reposition properly, affecting the nuclear reorientation process during wound healing. Also, defects are seen at the plasma membrane, where membrane protrusions are not formed properly during cellular migration.

These results indicate that a functional mechanical scaffold network is essential for coping with mechanical stress as well as with cell migration, and that lamin deficiency affects this network. Recent work from Brosig et al. and Lombardi et al. confirm that the LINC-complex (which connects to the nuclear lamina) is necessary for the correct nuclear repositioning and rotation [9-11].

Our data from chapter 3 shows a higher nuclear plasticity and associated increased chromatin mobility, which can affect genomic stability and thus influence premature aging.

In chapter 4 , we show that the loss of integrity of the nuclear membrane is a common finding in isolated dermal fibroblasts from a variety of laminopathy patients. We 
stipulate that the loss of lamin organization in laminopathies leads to a weakening of the nuclear scaffold. Weak spots in the nuclear envelope can lead to dynamic, reversible ruptures of the nuclear membrane at these locations. These ruptures are nonlethal to the cell, and result in a temporary loss of compartmentalization between the nucleus and the cytoplasm, resulting in a redistribution of signalling proteins and transcription factors that are normally transported actively across the nuclear membrane. While nuclear components that are associated with chromatin or other intranuclear structures will not diffuse out of the nucleus during the ruptures, (temporary) soluble proteins can diffuse out or into the nucleus. Similar results were seen in HIV-infected cells [12]. It is known that viruses modulate the nuclear lamina, inducing similar weak spots in the nuclear envelope, which can lead to the dynamic ruptures of the nuclear membrane [13-23].

In chapter 5, we concentrate on the presence of cytoplasmic PML nuclear body staining in a range of dermal fibroblasts from laminopathy patients. In general, patients with the most severe disease symptoms suffered from the highest numbers of cytoplasmic PLM-Nabs. Although this can partially be explained by the previous described phenomenon of nuclear ruptures, other mechanisms are believed to be involved in the mislocalization of PML bodies.

Over the years, many hypotheses concerning laminopathies have been formulated throughout the scientific community [24]. These hypotheses originate from different research fields in cell biology: cellular mechanics, signalling pathways and gene expression regulation.

The described hypotheses include:

1. The structural hypothesis

2. The gene expression hypothesis

3. The premature aging hypothesis

4. The cell proliferation hypothesis

5. The nuclear integrity hypothesis, as formulated in chapter 4 and 5

The structural hypothesis, stating that loss of structural strength causes laminopathies, is supported by our findings described in chapter 1 and 2 . We were able to demonstrate that the structure of physical connections between the different elements of the cellular scaffold (elements of the cytoskeleton, nuclear lamins and linker proteins) is compromised in diseased cells, leading to a disturbed nuclear reorientation upon scratch wound healing and cell migration. Apparently, if one of the cellular scaffold elements is aberrant, both structural and functional effects can be seen. 
Next to the pure mechanical function of the cellular scaffold network, this network also provides the infrastructure for response upon mechanical stress, through the activation of mechanosensitive signalling proteins [25]. This is called mechanosignalling. Mechanosignalling can become disturbed in case of a lamin mutation, which in turn can affect gene regulation. Lammerding et al. [25] showed that expression of mechanosensitive genes $e g r-1$ and iex-lin response to mechanical stimulation was impaired in Lmna-/- cells.

The second hypothesis states that these diseases arise from the loss of gene regulatory functions of lamin complexes While nuclear lamins are no transcription factors, they can act as a scaffold for gene regulation complexes [26]. An aberration in the nuclear lamina structure could thus affect the formation, localization and functioning of the complexes. Indications for impaired scaffold function come from studies examining the phosphorylation state of the transcription factor ERK.

Data from Muchir et al [27-30] and Emerson [31], as well as from our own research (see chapter 2) clearly indicate a constitutive increase in the amount of phosphorylated ERK in diseased cells. ERK1 and ERK2 interact with A-type lamins at the nuclear periphery and participate in the rapid regulation of activator protein 1 (AP-1) activity. Initially, it was suggested that the NE directly modulates ERK $1 / 2$ activity and downstream signaling, and that alterations in lamin $\mathrm{A} / \mathrm{C}$ expression might perturb $\mathrm{NE}$ structure sufficiently to directly affect these processes (see González et al [32]). However, a more recent study by Warren et al. [33] shows that nesprin-2 tethers ERK at PML-nuclear bodies,. As the nuclear localization of nesprin-2 depends on lamin A/C (chapter 2), it is conceivable that ERK can be retained in a lamin A/C/ERK/PML NB complex. This suggestion is even more interesting because of our discovery that PML NBs are often lost from the nucleus in laminopathies (chapter 4 and 5).

Other studies, which show a role of A-type lamins in transcription regulation, suggest that lamin $\mathrm{A} / \mathrm{C}$ deficiency abolishes TGF- $\beta 1$ induced $\mathrm{pRB}$ dephosphorylation through its interaction with PP2A. As a result, LMNA deficient cells display increased proliferation and decreased terminal differentiation [34]. Other pathways that were disturbed in case of lamin deficiency are the NFKB pathway and the activation of mechanosensitive genes iex-1 and egr-1, leading to an impaired mechanotransduction (see above) [25]. Expression of several other genes was suggested to be (negatively) regulated by lamins A/C in specific tissues, such as SREBP1 in adipocyte development [35], After treatment with protease inhibitors, prelamin A binds to SREBP1, which will lead to acquired lipodystrophy [36]. For reviews on the role of A-type lamins in the control gene expression see Andres et al [26] and Maraldi et al [37]. 
The third hypothesis states that the structure of the nuclear envelope is essential for the repair of damaged DNA. If the lamin structure is altered as a result of lamin deficiency or mutation, DNA repair fails and cells and tissues will age prematurely [38, 39]. Of course, extreme examples of premature aging can be found in the group of systemic diseases, including HGPS [40, 41]. However also in other laminopathies premature ageing of myoblasts can be observed. Also, an effect on proteasome inactivation (which is associated with cellular aging) was seen in the absence of lamins [42].

Another clue to the mechanism underlying premature ageing was found by GonzalezSuarez et al. [43, 44], who demonstrated that lamins are essential for the maintenance of telomeres. Loss of lamins leads to shortening of the telomeres and increased genomic instability $[45,46]$. In addition, a dramatic increase in telomere mobility within LMNA deficient cells suggest destabilization of chromatin organisation (Chapter 3)

The fourth hypothesis, the cell proliferation hypothesis, as described in [24], states that the loss of $\mathrm{Rb}$ function leads to an impaired satellite stem cell differentiation [47-49]. In normal cells A-type lamins form complexes with LAP2alfa to keep Rb in a dephosphorylated state. Absence of A-type lamins or lamin mutations disturb these complexes, leading to disturbance of the G1-S cell checkpoint control mechanism [50]. The inappropriate G1-S phase transition, allowing DNA duplication not preceded by DNA repair, could explain why in laminopathy cells so often DNA abnormalities arise during mitosis, as seen as a delay in the onset en progression of cytokinesis, and an impaired targeting of nuclear lamins into the nuclei of daughter cells [51]. Mutated lamins can also mislocalize during mitosis, leading to abnormal chromosome segregation and binucleation [52]. Disruption of mitosis leads to loss of proliferative capacity and premature aging [53].

Finally, the new hypothesis described by us, is based on our findings of nuclear ruptures in laminopathy cells. These ruptures, causing a sudden loss of compartmentalization between the nucleus and the cytoplasm, will disturb all existing gradients between the nucleus and the cytoplasm and allow proteins, which are under normal circumstances kept out of the nucleus, to diffuse into the nuclear interior and exert an untimely action. Not only unwanted transcription factors, but also protein modifying complexes (proteases, kinases, phosphatases etc.) and even organelles can enter into the ruptured nucleus (chapter 4 and 5) An overview of eukaryotic functions that involve a regulated nucleo-cytoplasmic compartmentalization is given in a review 
article from Carmo-Fonseca. These functions include the control of the cell cycle, signal transduction pathways (like the MAPK-pathway), stress response, muscle differentiation, and many others [54]. While it remains to be shown that nuclear ruptures indeed have a detrimental effect on tissues in patients, the occurrence of intranuclear inclusions of organelles in nuclei of patient's muscle and heart tissues indicates that indeed nuclear ruptures take place in vivo, and may be a major cause of disease development.

Given the large variety of laminopathies, it is well possible that not all hypotheses apply to all types of laminopathies, some of which affect one specific type of tissue (muscle, heart, adipose tissue), while other have a more systemic effect.

In laminopathies that affect muscle cells and other tissues that are under constant mechanical stress, the cellular pathology is probably mainly associated with the structural hypothesis, as the cells do no longer have a functional mechanical scaffold to withstand the constant tension and compression. In contrast, the premature ageing hypothesis applies mainly to progeroid diseases, although also in muscular dystrophies premature ageing of muscle cells has been encountered [42].

The gene expression hypothesis might be very important in lipodystrophies The inhibition of pre-adipocyte differentiation is the most probable cause by disturbed interaction with the transcription factors SREBP1 which is critically important for the differentiation of adipose tissue [35, 55-58].

When we carefully examine all the hypotheses formulated over the last years, most of which can be supported by solid evidence, we must conclude that lamins have multiple functions within the mammalian cells, and are linked to both structural and regulatory elements. When the nuclear lamina becomes dysfunctional, because of a structural defect, the connected networks (structural network, gene regulatory network, etc) are affected. When the nuclear membrane ruptures, it will affect both the gene regulation and the mechanical functioning of the cell.

Therefore, integration of the different hypotheses to form a holistic approach for the cellular mechanisms that underlie the several pathologies in the group of laminopathies will be necessary.

It is the combination of the different pathways, together with the specific features of the different affected tissues (skeletal muscle, adipose tissue, heart muscle and other tissues), that leads to the wide spectrum of diseases and clinical features ranging from very mild (no clinical symptoms) to very severe (death in utero), that are linked to a mutation in the lamin proteins. 
While this thesis mainly has shed light on the structural abnormalities in laminopathy cells, further research should focus on the link between cellular abnormalities, tissue damage, and prevention of or even treatment of disease development, based on our new knowledge on lamin function. 


\section{References}

[1] D. Fatkin, C. MacRae, T. Sasaki, M.R. Wolff, M. Porcu, M. Frenneaux, J. Atherton, H.J. Vidaillet, Jr., S. Spudich, U. De Girolami, J.G. Seidman, C. Seidman, F. Muntoni, G. Muehle, W. Johnson and B. McDonough, Missense mutations in the rod domain of the lamin $\mathrm{A} / \mathrm{C}$ gene as causes of dilated cardiomyopathy and conduction-system disease, N Engl J Med 341 (1999) 1715-1724.

[2] D.E. Ingber, Tensegrity I. Cell structure and hierarchical systems biology, J Cell Sci 116 (2003) 1157-1173.

[3] D.E. Ingber, Tensegrity II. How structural networks influence cellular information processing networks, J Cell Sci 116 (2003) 1397-1408.

[4] D.E. Ingber, Cellular mechanotransduction: putting all the pieces together again, Faseb J 20 (2006) 811-827.

[5] D.E. Ingber, Tensegrity and mechanotransduction, J Bodyw Mov Ther 12 (2008) 198-200.

[6] J.L. Broers, E.A. Peeters, H.J. Kuijpers, J. Endert, C.V. Bouten, C.W. Oomens, F.P. Baaijens and F.C. Ramaekers, Decreased mechanical stiffness in LMNA-/- cells is caused by defective nucleo-cytoskeletal integrity: implications for the development of laminopathies, Hum Mol Genet 13 (2004) 2567-2580.

[7] J.S. Lee, C.M. Hale, P. Panorchan, S.B. Khatau, J.P. George, Y. Tseng, C.L. Stewart, D. Hodzic and D. Wirtz, Nuclear lamin A/C deficiency induces defects in cell mechanics, polarization, and migration, Biophys J 93 (2007) 2542-2552.

[8] M. Schneider, W. Lu, S. Neumann, A. Brachner, J. Gotzmann, A.A. Noegel and I. Karakesisoglou, Molecular mechanisms of centrosome and cytoskeleton anchorage at the nuclear envelope, Cell Mol Life Sci.

[9] M. Brosig, J. Ferralli, L. Gelman, M. Chiquet and R. Chiquet-Ehrismann, Interfering with the connection between the nucleus and the cytoskeleton affects nuclear rotation, mechanotransduction and myogenesis, Int $\mathrm{J}$ Biochem Cell Biol 42 (2010) 1717-1728.

[10] M.L. Lombardi and J. Lammerding, Altered mechanical properties of the nucleus in disease, Methods Cell Biol 98 (2010) 121-141.

[11] M.L. Lombardi, D.E. Jaalouk, C.M. Shanahan, B. Burke, K.J. Roux and J. Lammerding, The interaction between nesprins and sun proteins at the nuclear envelope is critical for force transmission between the nucleus and cytoskeleton, J Biol Chem (2011).

[12] C.M. de Noronha, M.P. Sherman, H.W. Lin, M.V. Cavrois, R.D. Moir, R.D. Goldman and W.C. Greene, Dynamic disruptions in nuclear envelope 
architecture and integrity induced by HIV-1 Vpr, Science 294 (2001) 11051108.

[13] W. Muranyi, J. Haas, M. Wagner, G. Krohne and U.H. Koszinowski, Cytomegalovirus recruitment of cellular kinases to dissolve the nuclear lamina, Science 297 (2002) 854-857.

[14] A.E. Reynolds, L. Liang and J.D. Baines, Conformational changes in the nuclear lamina induced by herpes simplex virus type 1 require genes $U(L) 31$ and U(L)34, J Virol 78 (2004) 5564-5575.

[15] M. Marschall, A. Marzi, P. aus dem Siepen, R. Jochmann, M. Kalmer, S. Auerochs, P. Lischka, M. Leis and T. Stamminger, Cellular p32 recruits cytomegalovirus kinase pUL97 to redistribute the nuclear lamina, J Biol Chem 280 (2005) 33357-33367.

[16] R. Park and J.D. Baines, Herpes simplex virus type 1 infection induces activation and recruitment of protein kinase $\mathrm{C}$ to the nuclear membrane and increased phosphorylation of lamin B, J Virol 80 (2006) 494-504.

[17] S.L. Bjerke and R.J. Roller, Roles for herpes simplex virus type 1 UL34 and US3 proteins in disrupting the nuclear lamina during herpes simplex virus type 1 egress, Virology 347 (2006) 261-276.

[18] D. Camozzi, S. Pignatelli, C. Valvo, G. Lattanzi, C. Capanni, P. Dal Monte and M.P. Landini, Remodelling of the nuclear lamina during human cytomegalovirus infection: role of the viral proteins pUL50 and pUL53, J Gen Virol 89 (2008) 731-740.

[19] C.P. Lee, Y.H. Huang, S.F. Lin, Y. Chang, Y.H. Chang, K. Takada and M.R. Chen, Epstein-Barr virus BGLF4 kinase induces disassembly of the nuclear lamina to facilitate virion production, J Virol 82 (2008) 11913-11926.

[20] S. Hamirally, J.P. Kamil, Y.M. Ndassa-Colday, A.J. Lin, W.J. Jahng, M.C. Baek, S. Noton, L.A. Silva, M. Simpson-Holley, D.M. Knipe, D.E. Golan, J.A. Marto and D.M. Coen, Viral mimicry of Cdc2/cyclin-dependent kinase 1 mediates disruption of nuclear lamina during human cytomegalovirus nuclear egress, PLoS Pathog 5 (2009) e1000275.

[21] G.L. Cano-Monreal, K.M. Wylie, F. Cao, J.E. Tavis and L.A. Morrison, Herpes simplex virus 2 UL13 protein kinase disrupts nuclear lamins, Virology 392 (2009) 137-147.

[22] N.J. Buchkovich, T.G. Maguire and J.C. Alwine, Role of the endoplasmic reticulum chaperone $\mathrm{BiP}, \mathrm{SUN}$ domain proteins, and dynein in altering nuclear morphology during human cytomegalovirus infection, J Virol 84 7005-7017.

[23] N.R. Leach and R.J. Roller, Significance of host cell kinases in herpes simplex virus type 1 egress and lamin-associated protein disassembly from the nuclear lamina, Virology 406 127-137.

[24] J.L. Broers, F.C. Ramaekers, G. Bonne, R.B. Yaou and C.J. Hutchison, Nuclear lamins: laminopathies and their role in premature ageing, Physiol Rev 86 (2006) 967-1008. 
[25] J. Lammerding, P.C. Schulze, T. Takahashi, S. Kozlov, T. Sullivan, R.D. Kamm, C.L. Stewart and R.T. Lee, Lamin A/C deficiency causes defective nuclear mechanics and mechanotransduction, J Clin Invest 113 (2004) 370378.

[26] V. Andres and J.M. Gonzalez, Role of A-type lamins in signaling, transcription, and chromatin organization, J Cell Biol 187 (2009) 945-957.

[27] A. Muchir, P. Pavlidis, G. Bonne, Y.K. Hayashi and H.J. Worman, Activation of MAPK in hearts of EMD null mice: similarities between mouse models of $\mathrm{X}$-linked and autosomal dominant Emery Dreifuss muscular dystrophy, Hum Mol Genet 16 (2007) 1884-1895.

[28] A. Muchir, P. Pavlidis, V. Decostre, A.J. Herron, T. Arimura, G. Bonne and H.J. Worman, Activation of MAPK pathways links LMNA mutations to cardiomyopathy in Emery-Dreifuss muscular dystrophy, J Clin Invest 117 (2007) 1282-1293.

[29] A. Muchir, J. Shan, G. Bonne, S.E. Lehnart and H.J. Worman, Inhibition of extracellular signal-regulated kinase signaling to prevent cardiomyopathy caused by mutation in the gene encoding A-type lamins, Hum Mol Genet 18 (2009) 241-247.

[30] A. Muchir, W. Wu and H.J. Worman, Reduced expression of A-type lamins and emerin activates extracellular signal-regulated kinase in cultured cells, Biochim Biophys Acta 1792 (2009) 75-81.

[31] L.J. Emerson, M.R. Holt, M.A. Wheeler, M. Wehnert, M. Parsons and J.A. Ellis, Defects in cell spreading and ERK1/2 activation in fibroblasts with lamin A/C mutations, Biochim Biophys Acta 1792 (2009) 810-821.

[32] J.M. Gonzalez, A. Navarro-Puche, B. Casar, P. Crespo and V. Andres, Fast regulation of AP-1 activity through interaction of lamin A/C, ERK1/2, and cFos at the nuclear envelope, J Cell Biol 183 (2008) 653-666.

[33] D.T. Warren, T. Tajsic, J.A. Mellad, R. Searles, Q. Zhang and C.M. Shanahan, Novel nuclear nesprin-2 variants tether active extracellular signal-regulated MAPK1 and MAPK2 at promyelocytic leukemia protein nuclear bodies and act to regulate smooth muscle cell proliferation, J Biol Chem 285 (2010) 13111320.

[34] J.H. Van Berlo, J.W. Voncken, N. Kubben, J.L. Broers, R. Duisters, R.E. van Leeuwen, H.J. Crijns, F.C. Ramaekers, C.J. Hutchison and Y.M. Pinto, A-type lamins are essential for TGF-betal induced PP2A to dephosphorylate transcription factors, Hum Mol Genet 14 (2005) 2839-2849.

[35] C. Capanni, E. Mattioli, M. Columbaro, E. Lucarelli, V.K. Parnaik, G. Novelli, M. Wehnert, V. Cenni, N.M. Maraldi, S. Squarzoni and G. Lattanzi, Altered pre-lamin A processing is a common mechanism leading to lipodystrophy, Hum Mol Genet 14 (2005) 1489-1502.

[36] C.N. Goulbourne and D.J. Vaux, HIV protease inhibitors inhibit FACE1/ZMPSTE24: a mechanism for acquired lipodystrophy in patients on highly active antiretroviral therapy?, Biochem Soc Trans 38 (2010) 292-296. 
[37] N.M. Maraldi, C. Capanni, R. Del Coco, S. Squarzoni, M. Columbaro, E. Mattioli, G. Lattanzi and F.A. Manzoli, Muscular laminopathies: role of prelamin A in early steps of muscle differentiation, Adv Enzyme Regul 51 (2011) 246-256.

[38] A. Vaughan, M. Alvarez-Reyes, J.M. Bridger, J.L. Broers, F.C. Ramaekers, M. Wehnert, G.E. Morris, W.G.F. Whitfield and C.J. Hutchison, Both emerin and lamin $\mathrm{C}$ depend on lamin A for localization at the nuclear envelope, J Cell Sci 114 (2001) 2577-2590.

[39] B. Liu, J. Wang, K.M. Chan, W.M. Tjia, W. Deng, X. Guan, J.D. Huang, K.M. Li, P.Y. Chau, D.J. Chen, D. Pei, A.M. Pendas, J. Cadinanos, C. Lopez-Otin, H.F. Tse, C. Hutchison, J. Chen, Y. Cao, K.S. Cheah, K. Tryggvason and Z. Zhou, Genomic instability in laminopathy-based premature aging, Nat Med 11 (2005) 780-785.

[40] B. Liu and Z. Zhou, Lamin A/C, laminopathies and premature ageing, Histol Histopathol 23 (2008) 747-763.

[41] S. Pereira, P. Bourgeois, C. Navarro, V. Esteves-Vieira, P. Cau, A. De SandreGiovannoli and N. Levy, HGPS and related premature aging disorders: from genomic identification to the first therapeutic approaches, Mech Ageing Dev 129 (2008) 449-459.

[42] S. Kandert, M. Wehnert, C.R. Muller, B. Buendia and M.C. Dabauvalle, Impaired nuclear functions lead to increased senescence and inefficient differentiation in human myoblasts with a dominant p.R545C mutation in the LMNA gene, Eur J Cell Biol 88 (2009) 593-608.

[43] I. Gonzalez-Suarez, A.B. Redwood and S. Gonzalo, Loss of A-type lamins and genomic instability, Cell Cycle 8 (2009) 3860-3865.

[44] I. Gonzalez-Suarez and S. Gonzalo, Nurturing the genome: A-type lamins preserve genomic stability, Nucleus 1 (2010) 129-135.

[45] P. Therizols, C. Fairhead, G.G. Cabal, A. Genovesio, J.C. Olivo-Marin, B. Dujon and E. Fabre, Telomere tethering at the nuclear periphery is essential for efficient DNA double strand break repair in subtelomeric region, J Cell Biol 172 (2006) 189-199.

[46] I. Gonzalez-Suarez, A.B. Redwood, S.M. Perkins, B. Vermolen, D. Lichtensztejin, D.A. Grotsky, L. Morgado-Palacin, E.J. Gapud, B.P. Sleckman, T. Sullivan, J. Sage, C.L. Stewart, S. Mai and S. Gonzalo, Novel roles for A-type lamins in telomere biology and the DNA damage response pathway, Embo J 28 (2009) 2414-2427.

[47] C.J. Hutchison and H.J. Worman, A-type lamins: guardians of the soma?, Nat Cell Biol 6 (2004) 1062-1067.

[48] I. Mariappan and V.K. Parnaik, Sequestration of $p R b$ by cyclin D3 causes intranuclear reorganization of lamin $\mathrm{A} / \mathrm{C}$ during muscle cell differentiation, Mol Biol Cell 16 (2005) 1948-1960. 
[49] E. Markiewicz, M. Ledran and C.J. Hutchison, Remodelling of the nuclear lamina and nucleoskeleton is required for skeletal muscle differentiation in vitro, J Cell Sci 118 (2005) 409-420.

[50] N. Naetar and R. Foisner, Lamin complexes in the nuclear interior control progenitor cell proliferation and tissue homeostasis, Cell Cycle 8 (2009) 14881493.

[51] T. Dechat, T. Shimi, S.A. Adam, A.E. Rusinol, D.A. Andres, H.P. Spielmann, M.S. Sinensky and R.D. Goldman, Alterations in mitosis and cell cycle progression caused by a mutant lamin A known to accelerate human aging, Proc Natl Acad Sci U S A 104 (2007) 4955-4960.

[52] K. Cao, B.C. Capell, M.R. Erdos, K. Djabali and F.S. Collins, A lamin A protein isoform overexpressed in Hutchinson-Gilford progeria syndrome interferes with mitosis in progeria and normal cells, Proc Natl Acad Sci U S A 104 (2007) 4949-4954.

[53] T.V. Cohen, L. Hernandez and C.L. Stewart, Functions of the nuclear envelope and lamina in development and disease, Biochem Soc Trans 36 (2008) 1329-1334.

[54] M. Carmo-Fonseca, The contribution of nuclear compartmentalization to gene regulation, Cell 108 (2002) 513-521.

[55] D.J. Lloyd, R.C. Trembath and S. Shackleton, A novel interaction between lamin A and SREBP1: implications for partial lipodystrophy and other laminopathies, Hum Mol Genet 11 (2002) 769-777.

[56] R.L. Boguslavsky, C.L. Stewart and H.J. Worman, Nuclear lamin A inhibits adipocyte differentiation: implications for Dunnigan-type familial partial lipodystrophy, Hum Mol Genet 15 (2006) 653-663.

[57] N.M. Maraldi, C. Capanni, G. Lattanzi, D. Camozzi, A. Facchini and F.A. Manzoli, SREBP1 interaction with prelamin A forms: a pathogenic mechanism for lipodystrophic laminopathies, Adv Enzyme Regul 48 (2008) 209-223.

[58] V.L. Verstraeten, J. Renes, F.C. Ramaekers, M. Kamps, H.J. Kuijpers, F. Verheyen, M. Wabitsch, P.M. Steijlen, M.A. van Steensel and J.L. Broers, Reorganization of the nuclear lamina and cytoskeleton in adipogenesis, Histochem Cell Biol 135 (2011) 251-261. 
De celkern speelt een centrale rol in het functioneren van de cel. Het bevat het DNA, waaruit alle onderdelen van de cel gevormd en gereguleerd worden. Het DNA wordt omgeven door het kernmembraan.

De laatste jaren is echter duidelijk geworden dat de celkern geen losliggend vat vol DNA is, maar ook zowel fysiek als functioneel direct communiceert met haar omgeving. Een heel scala aan eiwitten is recent ontdekt die direct of indirect aan de celkern binden. Hierbij speelt de nucleaire lamina een belangrijke rol. De lamina ligt net onder het kernmembraan en bestaat uit een fijnmazig netwerk van lamine filamenten. Samen met het membraan en een aantal geassocieerde eiwitten vormt ze de nucleaire envelop.

Lamines zijn type $\mathrm{V}$ intermediaire filamenten. Ze kunnen worden onderverdeeld in twee hoofdtypes, de A-type lamines: lamines $\mathrm{A}, \mathrm{A} \Delta 10, \mathrm{C}$ en $\mathrm{C} 2$, gecodeerd door het lamine A/C gen en de B-type lamines: lamines B1, B2 en B3, gecodeerd door de lamine B1 en lamine B2 genen. De nucleaire lamina zorgt ervoor dat het kernmembraan een zekere stijfheid krijgt, waardoor de kern beter bestand is tegen externe mechanische krachten.

De kritische rol van lamines komt tot uiting wanneer de nucleaire lamina verstoord wordt door mutaties in het lamine $\mathrm{A} / \mathrm{C}$ gen. De pathologieën die dan ontstaan, worden laminopathieën genoemd. Op basis van ziekteverschijnselen die ze veroorzaken, kunnen we ze verdelen in verschillende groepen:

- Spierdystrofieën;

- Vetdystrofieën;

- Perifere neuropathieën;

- Progeroïde aandoeningen (vroegtijdige veroudering);

- Laminopathieën die andere weefsels aantasten.

In de loop der jaren zijn vele hypotheses over laminopathieën geformuleerd. Deze hypotheses zijn afkomstig uit verschillende onderzoeksgebieden in de celbiologie: cellulaire mechanica, signaaltransductie en genregulatie en -expressie.

De beschreven hypotheses zijn onder andere:

1. De structurele hypothese

2. De genexpressie hypothese

3. De voortijdige veroudering hypothese

4. De cel proliferatie hypothese

5. De nucleaire integriteit hypothese, zoals geformuleerd in hoofdstuk 4 en 5 
Het doel van dit proefschrift was om de structurele en de functionele gevolgen van lamine $\mathrm{A} / \mathrm{C}$ mutaties te onderzoeken in celkweken, afkomstig van huidfibroblasten van patiënten. Zo willen we de ontstaansmechanismen van laminopathieën beter leren begrijpen.

In hoofdstuk 1 beschrijven we de gevolgen van de afwezigheid van A-type lamines op de integriteit van de cellulaire mechanische structuur. Dit netwerk bestaat uit de nucleaire lamina (lamines en de bijbehorende eiwitten), het cytoskelet en cel adhesie moleculen. Normaal zijn al deze eiwitten met elkaar verbonden door middel van verbindingseiwitten. In de kernenvelop verbinden SUN-eiwitten en nesprines de nucleaire lamina met de verschillende elementen van het cytoskelet, die op hun beurt zijn verbonden met de buitenkant van de cel via catenine, vinculine, plectine en andere verbindingseiwitten. Het geheel vormt een netwerk dat fungeert als een z.g. tensigrity. Deze structuur is een combinatie van interne compressie (microtubuli) en spanningselementen (actine filamenten) en beschermt de cellulaire componenten (zoals de kern) tijdens externe mechanische krachten (bijvoorbeeld compressie). Alle elementen van dit mechanisch netwerk zijn essentieel, ze vormen samen één mechanische entiteit. Deze entiteit wordt in zijn geheel verstoord als een van de onderdelen verstoord is. Wanneer de lamina structuur wordt verstoord (als gevolg van een lamine $\mathrm{A} / \mathrm{C}$ mutatie of lamine verlies), heeft dit een dramatisch effect op de andere elementen van het netwerk. De lokalisatie van lamine geassocieerde eiwitten (bijvoorbeeld emerine), of verbindingseiwitten (bijvoorbeeld nesprines) is verstoord als de nucleaire lamina aangetast is.

Voorgaande studies toonden aan dat bij compressie kernen van cellen zonder lamine A/C kunnen openscheuren. Tegelijkertijd vertoonden deze cellen ook een verminderde weerstand tegen de compressie. Een uitbreiding van dit experiment op geïsoleerde kernen (niet langer verbonden met het cytoskelet) toonde aan dat een intact mechanisch netwerk nodig is om voldoende weerstand te genereren aangezien alle geïsoleerde kernen (met of zonder A-type lamines) vergelijkbare vervorming vertoonden bij de compressie.

In hoofdstuk 2 konden we aantonen dat het verlies van de integriteit van het mechanisch netwerk kan leiden tot een verlies van de functionele mechanische eigenschappen van de cel, zoals de reoriëntatie van de kern. De juiste positionering van de kern en het MTOC is essentieel voor het proces van cellulaire migratie. Deze herpositionering hangt af van het mechanisch netwerk. Als het netwerk verstoord 
wordt door een verstoring van de lamina, kan de kern en de MTOC zich niet correct herpositioneren tijdens cellulaire migratie. Dit leidt ook tot het niet correct hervormen van de plasma membraan tijdens cellulaire migratie (vorming van filopodia en plooien in het plasmamembraan.

Deze resultaten geven aan dat een functioneel mechanisch netwerk essentieel is voor de cel om te gaan met mechanische belasting tijdens cellulaire migratie, en dat een tekort aan functioneel lamine een invloed heeft op dit netwerk.

Onze gegevens uit hoofdstuk 3 tonen een hogere nucleaire plasticiteit van kernen zonder functioneel lamine $\mathrm{A} / \mathrm{C}$, wat leidt tot een toegenomen telomeer mobiliteit.

Progeria cellen, die progerine bevatten (gemuteerd lamine A/C), zijn juist minder elastisch en vertonen ook een lagere telomeer mobiliteit.

Een afwijkende genomische mobiliteit kan de stabiliteit van het genoom beïnvloeden, wat kan leiden tot vroegtijdige veroudering.

In hoofdstuk 4 beschrijven we een omkeerbaar, niet-lethaal verlies van integriteit van het kernmembraan in fibroblasten van verschillende laminopathie patiënten. We stellen vast dat het verlies van lamine organisatie in laminopathieën leidt tot een verzwakking van de mechanische structuur van de kern. Zwakke plekken in de nucleaire enveloppe kunnen leiden tot dynamische, omkeerbare scheuren in het kernmembraan op de plaats van de verzwakking. Deze breuken leiden niet tot celdood, maar resulteren in een tijdelijk verlies van de compartimentalisatie tussen kern en cytoplasma. Dit resulteert in een in- en uitstroom van signaaleiwitten en transcriptiefactoren die normaal gesproken enkel op een actieve manier door het kernmembraan heen getransporteerd kunnen worden. Andere nucleaire componenten die vasthangen aan chromatine of andere intranucleaire structuren diffunderen dan weer (bijna) niet uit de kern bij het optreden van een tijdelijke scheur in het kernmembraan. Een vergelijkbaar fenomeen werd ook al gezien bij HIV-geïnfecteerde cellen. Het is bekend dat virussen de nucleaire lamina moduleren en zwakke plekken induceren in de nucleaire envelop, wat kan leiden tot dynamische scheuren in het kernmembraan.

In hoofdstuk 5 concentreren we ons op de aanwezigheid van PML lichaampjes in het cytoplasma van fibroblasten van laminopathie patiënten. Normaal gezien komen deze lichaampjes enkel voor in de celkern. In het algemeen hebben patiënten met de meest ernstige ziekteverschijnselen de hoogste aantallen cytoplasmatische PML lichaampjes. Hoewel dit gedeeltelijk kan worden verklaard door het fenomeen van de nucleaire 
scheuren, zijn mogelijk ook andere mechanismen betrokken bij de mislokalisatie van PML lichaampjes.

Wanneer we zorgvuldig alle hypothesen onderzoeken die de afgelopen jaren geformuleerd zijn, en de verschillende onderzoeksresultaten die deze hypothesen ondersteunen, moeten we concluderen dat lamines meerdere functies hebben, en dat ze gekoppeld zijn aan zowel structurele als regulerende elementen. Wanneer de nucleaire lamina disfunctioneel wordt als gevolg van een mutatie in het lamine $\mathrm{A} / \mathrm{C}$ gen, beïnvloedt dit de verbonden netwerken (structureel netwerk, genregulerend netwerk, etc). Wanneer de kernmembraan scheurt, zal dit van invloed zijn op zowel de genregulatie en de mechanische werking van de cel.

Daarom is de integratie noodzakelijk van de verschillende hypotheses tot één holistische benadering voor de cellulaire mechanismen die ten grondslag liggen aan de verschillende pathologieën in de groep van laminopathieën.

Het is de combinatie van de verschillende trajecten, samen met de specifieke kenmerken van de verschillende aangetaste weefsels (spier, vetweefsel, hartspier e.a.), die leidt tot het brede spectrum van ziekten en klinische kenmerken, van heel mild (klinisch geen meetbare verschijnselen) tot heel dramatisch, (leidend tot voortijdige veroudering of plotseling hartfalen).

Hoewel dit proefschrift zich vooral richtte op de structurele afwijkingen in laminopathie cellen, is er meer inzicht verkregen in het mogelijk verband tussen cellulaire afwijkingen, en de beschadiging van het weefsel bij patiënten, waardoor er een betere voorspelling van de ziekteontwikkeling mogelijk is, en nieuwe wegen geopend worden voor de behandeling van deze ziekten. 


\section{Curriculum Vitae}

Frederik Houben was born on the 22nd of October 1979, in Herk-de-Stad (Belgium). The first twenty-four years he lived in Lummen, where he attended the Sint-Jozef primary school and Onbevlekt Hart van Maria secondary school. In 1997, he decided to study biology at the Limburgs Universitair Centrum (currently University Hasselt). In 1999, after obtaining his candidate in biology, he switched to biomedical sciences. In 2003, he graduated as master in biomedical sciences (magna cum laude) from the Transnational University Limburg (the cooperation between University Hasselt and Maastricht University). The day after graduation, he married Annelies Willem, and they moved to Aalter, as Annelies was studying medicine at the University Gent. He started working as environmental health specialist at OCL (Sint-Niklaas-Dendermonde, Belgium). After 2 years, they moved back to Limburg. Frederik applied for a PhDposition at Maastricht University (Netherlands) and (re)started his scientific career at the department of Molecular Cell Biology. From 2005 till 2009, he studied the effects of lamin deficiency on the mechanical properties of the cell and nucleus, under the guidance of Jos Broers and Frans Ramaekers.

In 2008, Frederik and Annelies adopted two children from Ethiopia, Tamirat (2004) and Mitu (2007). In 2009, he started working as coordinator cancer projects at Limburgs Gezondheidsoverleg vzw (Hasselt, Belgium). During his time here, two more children were born, Ayko and Briek (2010). In 2011, he moved from Hasselt to Sint-Truiden (Belgium) to start working at the Regional Hospital Sint-Trudo as coordinator of the patient information bureau. 


\section{List of publications}

1. Role of nuclear lamina-cytoskeleton interactions in the maintenance of cellular strength; Houben F, Ramaekers FC, Snoeckx LH, Broers JL; Biochim Biophys Acta. 2007 May;1773(5):675-686.

2. Disturbed nuclear orientation and cellular migration in A-type lamin deficient cells; Houben F, Willems CH, Declercq IL, Hochstenbach K, Kamps MA, Snoeckx LH, Ramaekers FC, Broers JL; Biochim Biophys Acta. 2009 Feb;1793(2):312-324.

3. Increased plasticity of the nuclear envelope and hypermobility of telomeres due to the loss of A-type lamins; De Vos WH, Houben F, Hoebe RA, Hennekam R, van Engelen B, Manders EM, Ramaekers FC, Broers JL, Van Oostveldt P; Biochim Biophys Acta. 2010 Apr;1800(4):448-458.

4. Repetitive disruptions of the nuclear envelope invoke temporary loss of cellular compartmentalization in laminopathies; De Vos WH, Houben F, Kamps M, Malhas A, Verheyen F, Cox J, Manders EM, Verstraeten VL, van Steensel MA, Marcelis CL, van den Wijngaard A, Vaux DJ, Ramaekers FC, Broers JL; Hum Mol Genet. 2011 Aug 25 


\section{Dankwoord}

Geachte professor Ramaekers, beste Frans. Ik ben zeer blij dat je mij de kans te geven om te kunnen promoveren. Je oog voor detail en logica hebben een grote meerwaarde betekent voor mijn werk. Bedankt voor de fijne en evenwichtige samenwerking gedurende de afgelopen jaren.

Jos, bedankt voor alles. Zes jaar van korte en lange vergaderingen in jouw kantoor, mails, gezamenlijke zoektochten naar dat ene antilichaam of die ene cellijn in het labo, de uren achter de vital of de confocale, de tochten naar Eindhoven in de eerste jaren, en af en toe zelfs de telefoongesprekken (met mate, want bellen naar het buitenland is duur), heeft ervoor gezorgd dat je voor mij veranderd bent van een 'baas' tot een compagnon de route. Jouw kinderlijk enthousiasme over onderzoek in het algemeen, en lamines in het bijzonder heeft me altijd geïnspireerd. En met kinderlijk bedoel ik niets negatiefs, het was een gedrevenheid en interesse voor onderzoek, die altijd primeerde op de andere aspecten. Je uitroep als een experiment een beloftevol resultaat opleverde ("Schrijf het op en stuur het naar Nature"), zonder enig cynisme of sarcasme, typeert dat dan ook ten volle.

Geachte professor Snoeckx, beste Luc. Iedere meeting met jou gaf mij weer de motivatie en energie die ik nodig had om door te gaan met mijn onderzoek. Je rustige en pragmatische aanpak hebben vaak mij terug op weg geholpen, op momenten dat ik het echt niet meer zag zitten. Onze overleg momenten waren (te) schaars, maar ze hebben mij enorm vooruit geholpen. En daar kan ik alleen maar heel dankbaar voor zijn.

Geachte professor Borgers, beste Marcel. Bedankt voor alle hulp met de EM-beelden. Ik zal nooit de dag vergeten waarop we samen besloten om de EM-beelden die je voor ons selecteerde, in te delen in 2 categorieën, die van voor mijn geboortejaar (1979!) en die van erna. Ook die keer dat je 40 (!) $\mathrm{kg}$ antischimmelcrème regelde toen ik een paar staaltjes vroeg voor het weeshuis dat ik ging bezoeken in Ethiopië, is zo'n moment om nooit te vergeten. Maar je inzicht in histologie en je gedrevenheid in het wetenschappelijk onderzoek zullen mij nog het meest bijblijven.

Uiteraard wil ik ook mijn Ersatzmoeders op MCB bedanken. Miriam, Helma, Mieke, Marie-Hélène, Francine en Yolanda, jullie hebben mij gedurende die 4 jaar dat ik op MCB zat, veel geleerd. Streng als het nodig was (bijvoorbeeld als ik weer eens 
zondigde tegen één of ander protocol), maar altijd behulpzaam als ik voor de zoveelste keer vastzat met een experiment, als een toestel niet wilde doen wat ik wilde, of als de administratie van de universiteit moeilijk deed. De discussies over lade vs schuif nam ik er dan ook graag bij.

Ook de rest van MCB waren op tijd en stond een grote steun en toeverlaat, Bert (bedankt voor al de western blot tips), Wiel (als volledige computer-leek heb ik vaak genoeg een beroep op je moeten doen), Fons (voor alle EM-opnames) en alle anderen, hartelijk bedankt.

Tal van studenten hebben de revue gepasseerd tijdens de jaren op MCB. Ik moet eerlijk toegeven dat het vooral de studenten tijdens de eerste jaren mij het meest zijn bijgebleven. Coen (in het begin leek het wel of jij de AIO was en ik de student), Kevin, Connie, Nuram, Inge, Maarten en Guido, het was fijn om met jullie samen te werken.

Mijn collega-AIO's, bedankt om iedere dag weer naar mijn geklaag en gezaag te luisteren. Ook de traploopwedstrijdjes zal ik niet snel vergeten. Ik weet niet waar jullie nu allemaal zitten, maar ik wens jullie het allerbeste.

Wendy, Ronald, Karly, Paula, Valerie, Nadine en Dorian, bedankt voor de fijne tijd.

Winnok, de laatste jaren is de samenwerking tussen Gent en Maastricht steeds intensiever geworden. Jouw bijdrage aan ons project is ook steeds groter en groter geworden, en zorgde voor een belangrijke uitdieping en onderbouwing van onze onderzoeksresultaten. Bedankt daarvoor. Hopelijk ben je ondertussen al wat gewend aan de Maastrichtse stijl.

Mijn carpoolcollega's, Kristiaan, Tim en (voor een zeer korte tijd) Stani, het was mij een genoegen. Ik zal onze bijnamen maar niet vermelden, er lezen immers ook serieuze mensen mee. Ik heb 1 ding van jullie geleerd, haal nooit in met een oude Ford. Ondertussen zitten jullie allebei weer in Maastricht als post-doc. Ik wens jullie nog veel succes in jullie onderzoekscarrière en een fijn leven met jullie echtgenotes en kindjes.

Mijn ouders hebben mij altijd gesteund, tijdens mijn eerste studie, tijdens mijn tweede studie, tijdens mijn promotiejaren, en alle momenten daarvoor, tussen en erna. Ma en pa, bedankt! 
Mijn 2 paranimfen, eerst en vooral Miriam. Het aantal kleuringen, tellingen en andere experimenten die je voor mij gedaan hebt, is schier eindeloos. Zonder jou was dit proefschrift er nooit kunnen komen. En dan ook nog mijn vader. Ik zou niet weten wie beter geschikt zou zijn als mijn paranimf.

Zoals in een goed artikel staan de belangrijkste mensen achteraan. Annelies, toen ik je al die jaren geleden leerde kennen, waren we gewoon twee studenten. Toen we in 2005 terug naar Limburg kwamen, waren we nog altijd maar met twee, maar dan wel getrouwd. Nu zijn we niet meer met twee. Zes is de (voorlopige?) eindstand, met Tamirat, Mitu, Ayko en Briek is ons gezinnetje een heel gezin geworden. Bedankt om deze weg met mij te bewandelen, hopelijk mag de tocht nog heel lang duren. 